\title{
Designing gradient plotted scaffolds as a tool for improving osteochondral regeneration
}

Citation for published version (APA):

Di Luca, A. (2017). Designing gradient plotted scaffolds as a tool for improving osteochondral regeneration. [Doctoral Thesis, Maastricht University]. Gildeprint Drukkerijen. https://doi.org/10.26481/dis.20170608adl

Document status and date:

Published: 01/01/2017

DOI:

10.26481/dis.20170608adl

Document Version:

Publisher's PDF, also known as Version of record

\section{Please check the document version of this publication:}

- A submitted manuscript is the version of the article upon submission and before peer-review. There can be important differences between the submitted version and the official published version of record.

People interested in the research are advised to contact the author for the final version of the publication, or visit the DOI to the publisher's website.

- The final author version and the galley proof are versions of the publication after peer review.

- The final published version features the final layout of the paper including the volume, issue and page numbers.

Link to publication

\footnotetext{
General rights rights.

- You may freely distribute the URL identifying the publication in the public portal. please follow below link for the End User Agreement:

www.umlib.nl/taverne-license

Take down policy

If you believe that this document breaches copyright please contact us at:

repository@maastrichtuniversity.nl

providing details and we will investigate your claim.
}

Copyright and moral rights for the publications made accessible in the public portal are retained by the authors and/or other copyright owners and it is a condition of accessing publications that users recognise and abide by the legal requirements associated with these

- Users may download and print one copy of any publication from the public portal for the purpose of private study or research.

- You may not further distribute the material or use it for any profit-making activity or commercial gain

If the publication is distributed under the terms of Article $25 \mathrm{fa}$ of the Dutch Copyright Act, indicated by the "Taverne" license above, 


\section{Designing gradient plotted scaffolds as a tool for \\ improving osteochondral regeneration}

Andrea Di Luca 
ISBN: 978-94-6233-633-9

(c) Copyright 2017, Andrea Di Luca. All rights reserved.

Cover design: Giulia Marchioli

Printed by: Gildeprint

The research described in this thesis was sponsored by:
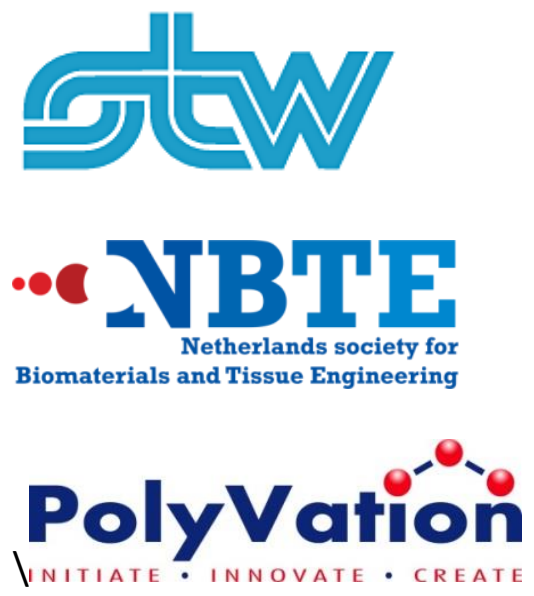


\section{Designing gradient plotted scaffolds as a tool for improving osteochondral regeneration}

\section{DISSERTATION}

To obtain the degree of Doctor at the Maastricht University,

on the authority of the Rector Magnificus,

Prof.dr. Rianne M. Letschert

In accordance with the decision of the Board of Deans,

to be defended in public

on Thursday, June $8^{\text {th }} 2017$, at $16: 00$

by

Andrea Di Luca

Born on July $27^{\text {th }}, 1984$

In Trieste, Italy 


\section{Promotors:}

Prof.dr. C.A. van Blitterswijk Maastricht University

Prof.dr. L. Moroni

Maastricht University

\section{Assessment Committee:}

1. Prof.dr. M. Post(Chairman) Maastricht University, The Netherlands

2. Prof.dr. P. Kessler

Maastricht University, The Netherlands

3. Prof.dr. S. Rastogi

Maastricht University, The Netherlands

4. Dr. J. van Beucken

Radboud University Nijmegen,

The Netherlands

5. Dr. R. van Donkelaar

Technical University of Eindhoven,

The Netherlands 


\section{Table of content}

\section{STATEMENTS}

\section{GENERAL INTRODUCTION AND SUMMARY}

CHAPTER 1 The osteochondral interface as a gradient tissue: from development to the fabrication of gradient scaffolds for regenerative medicine

CHAPTER 2 Gradients in pore size enhance the osteogenic differentiation of human mesenchymal stromal cells in three-dimensional scaffolds

CHAPTER 3 Influencing chondrogenic differentiation of human mesenchymal stromal cells in scaffolds displaying a structural gradient in pore size

CHAPTER 4 Toward mimicking the bone structure: design of novel hierarchical scaffolds with a tailored radial porosity gradient

CHAPTER 5 Tuning Cell Differentiation into a 3D Scaffold Presenting a Pore Shape Gradient for Osteochondral Regeneration

CHAPTER 6 Surface energy and stiffness discrete gradients in additive manufactured scaffolds for osteochondral regeneration

CHAPTER 7 Creeping Proteins in Microporous Structures: Polymer Brush-Assisted Fabrication of 3D Gradients for Tissue Engineering 
CHAPTER 8 Binding growth factors to 3D plotted scaffolds: are covalent biological gradients an option for osteochondral tissue regeneration? 223

$\begin{array}{lll}\text { CHAPTER } 9 & \text { General discussion } & 249\end{array}$

$\begin{array}{lll}\text { CHAPTER } 10 \text { Valorization } & 261\end{array}$

$\begin{array}{ll}\text { SAMENVATTING } & 266\end{array}$

$\begin{array}{ll}\text { CURRICULUM VITAE } & 269\end{array}$

$\begin{array}{ll}\text { ACKNOWLEDGMENT } & \mathbf{2 7 0}\end{array}$

$\begin{array}{ll}\text { PUBLICATIONS } & 273\end{array}$ 


\section{STATEMENTS}

With the thesis

Designing gradient plotted scaffolds as a tool for improving osteochondral regeneration 
1 It's not necessary to engineer our body, we rather should improve and exploit the regeneration system already present in our body nature. [This thesis]

2 The human body is composed of organs, which are composed of tissues. To proceed from one tissue to another a gradual passage is ensured by gradients. Hence, gradients can be seen as key components of our body. This thesis is based on the regeneration of one of these transition tissues, the osteochondral interface. [This thesis]

3 The bioactivity of the scaffold does not rely on the addition of bioactive molecules: structural properties can be as strong as soluble factors. [This thesis]

$4 \quad 3 D$ printing has entered in our daily life, with shops able to make your desired 3D printed object. You can buy your own 3D printing machine today. Even Uncle Scrooge asked Gyro Gearloose to provide every citizen of Duckburg with a 3D printing machine. [This thesis/Zio Paperone (Uncle Scrooge) e i prodigi della 3D pi - Topolino nr 3062]

5 In regenerating the osteochondral interface, we should skip the ex-vivo step and aim at single-step procedures for the wellbeing of the patients, using the regeneration capacity of stromal cells present in our bone marrow. [This thesis]

6 If we think about the knowledge gathered during the human history we may be really impressed, but if we think about what we don't know yet we can conclude that rather than having waded deep into the sea of knowledge we have merely dipped our toes in the water. [Michael Hanlon - 10 questions science cannot answer (yet)]

7 Science has become something like politics used to be, a vast ocean of shifting opinions, tides of fashion that wash in and out. [Michael Hanlon - 10 questions science cannot answer (yet)]

8 Well, here at last, dear friends, on the pages of this thesis comes the end of my PhD in Twente and Maastricht. Go in peace! I will not say: do not weep for not all tears are an evil - [Rearranged from Gandalf, J.R.R. Tolkien, The Grey Havens, The Return of the King] 


\section{General introduction and summary}

Gradients are present in the human body connecting tissues with different properties. Examples are the skin from the deep layers to the higher stratum corneum or long bones, which increase the compactness of the matrix in the radial direction. Another example is represented by interface tissues. In case of skeletal tissues, examples comprise interfaces between ligaments and bone or between cancellous bone and hyaline cartilage.

Among graded tissues in our body, the osteochondral tissue is of high importance due to the continuously ageing of our society and the consequent increase in accidents and degenerative diseases involving the skeletal system. The osteochondral tissue is the tissue characterized by the transition between trabecular bone and hyaline cartilage. Along this tissue several features are changing gradually to allow a smooth transition from the hard and mineralized subchondral bone to the softer hyaline cartilage above it. To generate such a transition between two tissues with differences in many characteristics, several gradients are recognizable when moving from the bone side to the joint surface. The degree of mineralization and stiffness decreases gradually from the subchondral bone toward the surface of the joint. Additionally, within hyaline cartilage collagen II, chondrocyte number and glucose concentration increase from the deep layer toward the surface whereas Glycosaminoglycan (GAG) stiffness and chondrocyte size show an opposite trend.

Considering these gradual variations, an appealing strategy to regenerate the osteochondral tissue could pass from the designing and fabrication of threedimensional (3D) scaffolds displaying such structural, physico-chemical and biological gradients. Additive manufacturing has gained a lot of interest in the past decades as a fabrication technology platform for its possible application, among others, in the field of tissue engineering and regenerative medicine. Additive manufactured (AM) scaffolds present a fully interconnected pore network and a desired shape; scaffold characteristics such as fiber size, pore size and geometry, and material composition can be tuned during the plotting. The resulting scaffolds 
can be afterwards further functionalized to incorporate bioactive cues to promote cell adhesion and/or differentiation.

In the present thesis, bone marrow derived human mesenchymal stromal cells (hMSCs) were chosen as a cell model, envisioning that they could be combined to the engineered scaffolds directly during surgery via clinical procedures like microfracture. These cells were seeded on AM scaffolds with different in-built gradients and their differentiation profile assessed toward the osteogenic and chondrogenic lineage to evaluate the efficacy of the designed 3D gradients. Additive manufacturing was used to produce scaffolds presenting gradients in pore size, geometry, physico-chemical properties and bioactive molecules.

In chapter 1, the osteochondral interface is presented as a gradient tissue, introducing from a developmental biology perspective how the tissue composition varies from the subchondral bony side to the cartilage side, and a review of the most recent scaffold-based treatment using gradient structures is discussed.

In chapter 2, we analyzed the differentiation of hMSCs toward the osteogenic lineage when cultured in a scaffold with an in-built gradient in pore size. Alkaline phosphatase (ALP) activity, the expression of the genetic markers Runx2, Bone sialoprotein, osteocalcin, osteopontin, and ALP, and the presence of mineralization nodules were analyzed to understand if the gradient has a beneficial effect on hMSC differentiation compared to non-gradient homogeneous structures. The same markers were analyzed to see if cells residing in different gradient compartments displayed a differential osteogenic profile.

In chapter 3, the differentiation of hMSCs toward the chondrogenic lineage was analyzed in a gradient displaying the same structural characteristic as the one described in chapter 2. As chondrogenic markers, glycosaminoglycan (GAG) production and the genetic markers sox 9 , aggrecan and collagen type II were chosen. Again, the effect of the gradient scaffolds on hMSC chondrogenic differentiation was compared to scaffolds presenting a homogenous pore size and within the gradient compartments. GAGs are structural proteoglycan deposited by chondrocytes and hMSCs under differentiation toward the chondrogenic lineage. 
Their presence is a sign of differentiation and can originate from cell number or cell density. In order to decouple and analyze separately these possible causes, GAG amount was normalized by cell number and pore volume in order to link the scaffolds' structural properties with hMSC differentiation.

In chapter 4 the porosity gradient was generated in the radial direction. Long bones present a gradient in the radial direction, from outside to inside first the compact bone, followed by the trabecular bone and the bone marrow channel. Scaffolds mimicking the porosity of the 3 regions were plotted and the hMSC differentiation profile toward the osteogenic lineage was analyzed. The mechanical properties of the scaffolds were characterized and hMSC osteogenic differentiation was analyzed in terms of genetic and biochemical markers, while histochemical staining and x-ray diffraction were used to determine the presence of mineralization.

In chapter 5, the response of hMSC differentiation within scaffolds presenting a pore shape gradient along the $z$ axis was assessed. Pore shape gradient (PSG) scaffolds were plotted by varying the angle between the fibers of consequent layers. The layers orientation 0-90 formed squared pores, 0-45 rhomboidal pores, 0-30 and 0-15 formed rhomboidal pores with increasingly smaller minor diagonal and larger major diagonal. The differentiation of hMSCs toward the osteogenic and chondrogenic lineage was analyzed biochemically and genetically. Osteogenic and chondrogenic markers were analyzed in gradient scaffolds with respect to scaffolds presenting a homogenous pore size as well as between pore geometries within the same scaffold.

In chapter 6, scaffolds displaying physico-chemical gradients were analyzed. By plotting 3 materials within the same scaffolds, gradients in stiffness and surface energy were achieved. These scaffolds were first characterized and the gradients were profiled along the z-axis. The hMSC differentiation towards the osteogenic and chondrogenic differentiation was analyzed in response to these gradients. 
In chapter $\mathbf{7}$ the functionalization of AM scaffolds with nanobrushes used as linkers between the scaffold surface and bioactive proteins was developed. After the functionalization with nanobrushes a simple method to obtain a gradient in bioactive molecules was presented. To prove the biological activity of such a gradient, a scaffolds functionalized with fibronectin was used to drive cell attachment onto the scaffolds following a radial and an axial gradient.

In chapter 8, AM scaffolds were functionalized with BMP-2 and TGF- $\beta 3$. The effect of the functionalized scaffolds on hMSC differentiation was compared to cells growing on a non-functionalized scaffold with media supplemented with the soluble growth factor. Scaffolds displaying a gradient of these growth factors were generated and their effect on hMSC differentiation was evaluated.

In chapter 9, all the scaffolds described in this thesis are compared with each other, further studies are proposed to further understand the possible beneficial influence of gradient scaffolds for osteochondral regeneration, and their use in regenerative medicine placed in perspective. 


\section{CHAPTER 1}

"The osteochondral interface as a gradient tissue: from development to the fabrication of gradient scaffolds for regenerative medicine"

Andrea Di Luca, Clemens Van Blitterswijk, Lorenzo Moroni

Published on Birth Defects Res C Embryo Today. 2015 Mar;105(1):34-52 


\section{Abstract}

The osteochondral interface is not only the interface between two tissues, but also the evolution of hard and stiff bone tissue to the softer and viscoelastic articular cartilage covering the joint surface. In order to generate a smooth transition between two tissues with such differences in many of their characteristics, several gradients are recognizable when moving from the bone side to the joint surface. It is, therefore, necessary to implement such gradients in the design of scaffolds to regenerate the osteochondral interface, so to mimic the anatomical, biological and physico-chemical properties of bone and cartilage as closely as possible. In the past years several scaffolds were developed for osteochondral regeneration: biphasic, tri-phasic, and multi-layered scaffolds were used in order to mimic the compartmental nature of this tissue. The structure of these scaffolds presented gradients in mechanical, physico-chemical or biological properties. The use of gradient scaffolds with already differentiated or progenitor cells have been recently proposed. Some of these approaches have been also translated in clinical trials, yet without the expected satisfactory results, thus suggesting that further efforts in the development of constructs which can lead to a functional regeneration of the osteochondral interface by presenting gradients more closely resembling its native environment will be needed in the near future. The aim of this review is to analyze the gradients present in the osteochondral interface from the early stage of embryonic life up to the adult organism and give an overview of the studies which involved gradient scaffolds for its regeneration. 


\section{Introduction}

Osteoarthritis (OA) nowadays represent worldwide, a common degenerative disease in old people with a high socio economic impact. It has been estimated that $40 \%$ of the population over 65 years presents symptomatic $O A$ in large joints, consequently affecting the quality of life of elderly populations [1-3]. The increase of life expectancy will eventually increase the percentage of population presenting $\mathrm{OA}$. Clinically, current therapies to regenerate osteochondral $(\mathrm{OC})$ tissues are not yet completely successful. All the available treatments such as reparative surgery, allografts, autografts and the implantation/transplantation of autologous chondrocytes, besides limitations like the formation of fibrocartilage and lack of donor supply, inflict further tissue damage before any therapeutic effect can be achieved $[4,5]$. Due to the fore mentioned reasons, major efforts in regenerative medicine have been placed in the past few years to present new solutions that hold the potential to improve the outcome of current therapies. Scaffold-based regenerative medicine strategies, in particular, have found a lot of applications in the past decades in skeletal tissue engineering, due to their ability to support cells and tissue growth in $3 \mathrm{D}$ and to mimic to some extent the extracellular matrix (ECM) architectural properties and composition.

The osteochondral tissue is located at the end of long bones and allows the transition from bone to cartilage. Since these two anatomical structures present significantly different characteristics at the macro, micro, and nano scales in terms of structural, mechanical, physico-chemical and biological properties, an interfacial tissue that shows a gradual variation of these features at different scales is necessary. Due to the fine interplay between the bone and the cartilage side, the two compartments cannot be separated since they are tightly interconnected not only under physiological conditions, but also during the progression of OA. When thinking about the regeneration of the osteochondral tissue, designing scaffoldbased regenerative strategies that can take into consideration such graded variations of the native tissue properties seems, therefore, a promising route. In the past few years a number of scaffolds presenting either a bi- or tri-phasic [6-11] structure were proposed for the regeneration of the $\mathrm{OC}$ tissue in vitro and in vivo. 
Constructs presenting a discrete or continuous gradients in geometry [12], stiffness [9], biochemical composition $[9,13]$ at the macro and micro scale, as well as gradients in growth factor concentrations [14-16] can be found in literature. The aim of this review is to dissect the gradient nature of the $O C$ tissue, such as its changes in ECM molecular composition and orientation, the resulting variation in physicochemical and mechanical properties, as well as in the cellularity, nutrient availability and growth factors involved in its development, This knowledge is then linked to the strategies applied so far in regenerative medicine for $\mathrm{OC}$ tissue regeneration.

\section{Osteochondral tissue development}

From a developmental point of view, bone and cartilage of appendicular bones arise from the lateral plate mesoderm. The first step in their development is the condensation of osteochondral progenitor cells into aggregates under the effect of TGF- $\beta$, which promotes the expression of molecules involved in the condensation process such as $\mathrm{N}$-cadherin, neural cella adhesion molecule (N-CAM), fibronectin, and Tenascin- $\mathrm{C}[17,18]$. The condensation determines the formation of a central part of cells, which continue to proliferate and express the transcription factor sex determining region Y-box 9 (sox-9), and a population of cells located in the periphery of the aggregates known as the perichondrium [19]. Sox-9 is expressed in all the chondrocytes with exception of hypertrophic chondrocytes [20]. The perichondrium functions as a reservoir of chondrocytes during bone development, which will progress until bone formation. Under the influence of a growth factor cocktail, including amongst others insulin growth factor -1 , fibroblast growth factor 2 (FGF-2) and bone morphogenetic proteins (BMPs) -2, -4, -7, and -14 [17, 21, 22], the cells within the aggregates proceed to chondroblasts and begin to produce aggrecan and collagen type II, IX and XI. Chondroblasts organize into a structure called growth plate, from which bone will be generated. The central part of the growth plate is called primary ossification center and constitutes the first cartilage portion replaced by bone. The growth plate is responsible of the growth in length of appendicular bones during life. Within the growth plate different zones can be 
identified, each one characterized by a pool of chondrocytes displaying differences in size, proliferation rate and ECM deposition. At the distal end of long bones, chondrocytes appear small and rounded. Chondrocytes are located in the so called resting zone, which has the function of providing further cells that will continue in the maturation process. Once stimulated, resting zone chondrocytes interact with the surrounding ECM, assume a flat phenotype and begin the formation of the columnar zone. Near the top of the columnar zone, cells display the highest proliferation rate [23]. Cell proliferation is maintained by the action of parathyroid hormone-related peptide PTHrP, which is produced by periarticular chondrocytes and negatively regulates terminal cell differentiation [17, 24]. As we move from the periarticular zone into the resting and columnar zones, chondrocytes move away from the PTHrP source, arrest their proliferation and undergo prehypertrophic differentiation at the bottom of the columnar zone. Prehypertrophic chondrocytes produces indian hedgehog $(\mathrm{IHH})$, which stimulates on one side PTHrP synthesis by periarticular chondrocytes, and on the other side cell terminal differentiation and the hypertrophic zone formation. Their volume increases by 20 fold [25] and their ECM synthesis switches from mainly collagen type II to collagen type $X$ formation. The further chondrocyte developmental stage is the late hypertrophic chondrocyte, which expresses some pre osteoblast markers such as MMP-13, known to promote vascular invasion and the consequent progressive replacement of cartilage by bone. Late hypertrophic chondrocytes represent the terminal stage of differentiation in the chondrogenic cell lineage [26]. The final fate of hypertrophic chondrocytes is apoptosis, but cells which escape death become osteoblasts [27]. The cells of the perichondrium flanking the hypertrophic zone become osteoblasts and form the periosteum under the effect of Runt-related transcription factor 2 (runx-2). The hypertrophic chondrocyte begins to express runx-2, an early marker for osteogenesis and continue to be expressed in osteoblasts. Blood vessel and osteoblasts from the newly formed bone invade the hypertrophic region and replace cartilage with bone and bone marrow [17].

Many of the events in chondrogenesis, from early differentiation of hMSCs in prechondrocytes to the evolution toward the hypertrophic stage, are governed by the Wnt signaling pathway. This pathway can follow two distinct route named Wnt 
"canonical" and "non-canonical" pathways. In the $\beta$-catenin or canonical pathway, Wnt binds its receptor Frizzled, which activate glycogen synthase kinase-3 $\beta$ (GSK$3 \beta$ ) that determines the phosphorylation of $\beta$-catenin. In its phosphorylated form $\beta$ catenin is stable and accumulates in the cytoplasm. Subsequently, $\beta$-catenin translocates to the nucleus and interacts with the gene expression regulatory apparatus [28]. The non-canonical pathway is $\beta$-catenin independent and based on intracellular calcium levels. The binding of Wnt with Frizzled stimulates the release of intracellular $\mathrm{Ca}^{++}$and the activation of protein kinase $\mathrm{C}$ and $\mathrm{Ca}^{2+}$-calmodulindependent protein kinase II, which are involved in ventral patterning and regulation of cell adhesion, migration and tissue separation [29, 30]. In contrast to what happens in the growth plate, articular cartilage is highly resistant to the hypertrophic differentiation [31]. The control of chondrocytes differentiation relies on the expression of antagonist of the Wnt signaling pathway such as Gremlin 1, Frizzled-Related Protein, and Dickkopf-1 (Dkk-1) [32]. Beside the Wnt family another pool of protein actively involved in chondrogenic and osteogenic development is represented by the transforming growth factor $\beta$ (TGF- $\beta$ ) superfamily. Within TGF- $\beta$ superfamily, BMPs and TGF- $\beta$ s are the most important growth factors known to regulate osteogenic and chondrogenic cell differentiation [33]. There are three TGF- $\beta$ isoforms, namely TGF- $\beta$ 1, 2 and 3. TGF- $\beta 1$ and TGF$\beta 3$ are related to chondrogenesis. The effectors of the canonical pathways are the small mother against decapentaplegic proteins (Smads), a pool of cytoplasmatic proteins able to form complexes which translocate in the nucleus and act as transcription factors [34]. Smad-1, $-2,-3,-5$ and -8 are effectors of the TGF- $\beta$ and BMP pathways. Smad-4 act as co-factor forming the complex which translocate in the nucleus, whereas Smad-6 and -7 have an inhibitory activity [35]. In TGF- $\beta$ pathway, one TGF- $\beta$ isoform binds a type II dimeric receptor, which recruits a dimeric type I receptor generating a heterotetrameric complex. This complex presents a kinase intracellular domain, type II receptor phosphorylates type I receptor [34], which phosphorylate Smad-2 or Smad-3. This event causes the formation of the Smad2/3-Smad4 complex, its translocation in the nucleus and the action as transcription factor, increasing the Sox9-dependent transcriptional activity and the transcription of collagen Ila $[36,37]$. BMP family presents also several 
isoforms. BMP-2, -6 , and -7 are known to promote osteogenic differentiation whereas BMP-3 act as an inhibitor. The BMP pathway presents the same event sequence. The BMP binds a heterocomplex composed by a type I and a type II receptor. Binding generates the recruitment of another couple of receptors in order to form the heterotetrameric complex. Type II receptor phosphorylates type I receptor which phosphorylates Smad-1, -5 or -8 [38]. This event causes the formation of the Smad-1/5/8-Smad-4 complex, which translocates into the nucleus determining the transcription of Runx2, Collagen type-la, alkaline phosphatase, and osteocalcin [38].

\section{The components of osteochondral tissue}

The OC tissue is composed by two main compartments, subchondral bone and articular cartilage [39]. Within these compartments, a further division in areas can be performed. Beside the subchondral bone plate, the calcified cartilage is often considered part of it; the line defining the passage to the articular cartilage is the so called tide mark. Articular cartilage can be further divide into 3 regions: (i) the radial zone, standing on top of mineralized cartilage; (ii) the transition zone, central in the cartilage tissue; (iii) and the superficial zone, interfacing with the synovial fluid and the joint space. The OC tissue has an height of $3 \mathrm{~mm}$ approximately in adults, of which about $90 \%$ consists of articular cartilage, $5 \%$ by calcified cartilage and $5 \%$ by the subchondral bone plate [40]. A schematic representation and histological section of the components of the $\mathrm{OC}$ tissue are shown in Figure 1. 

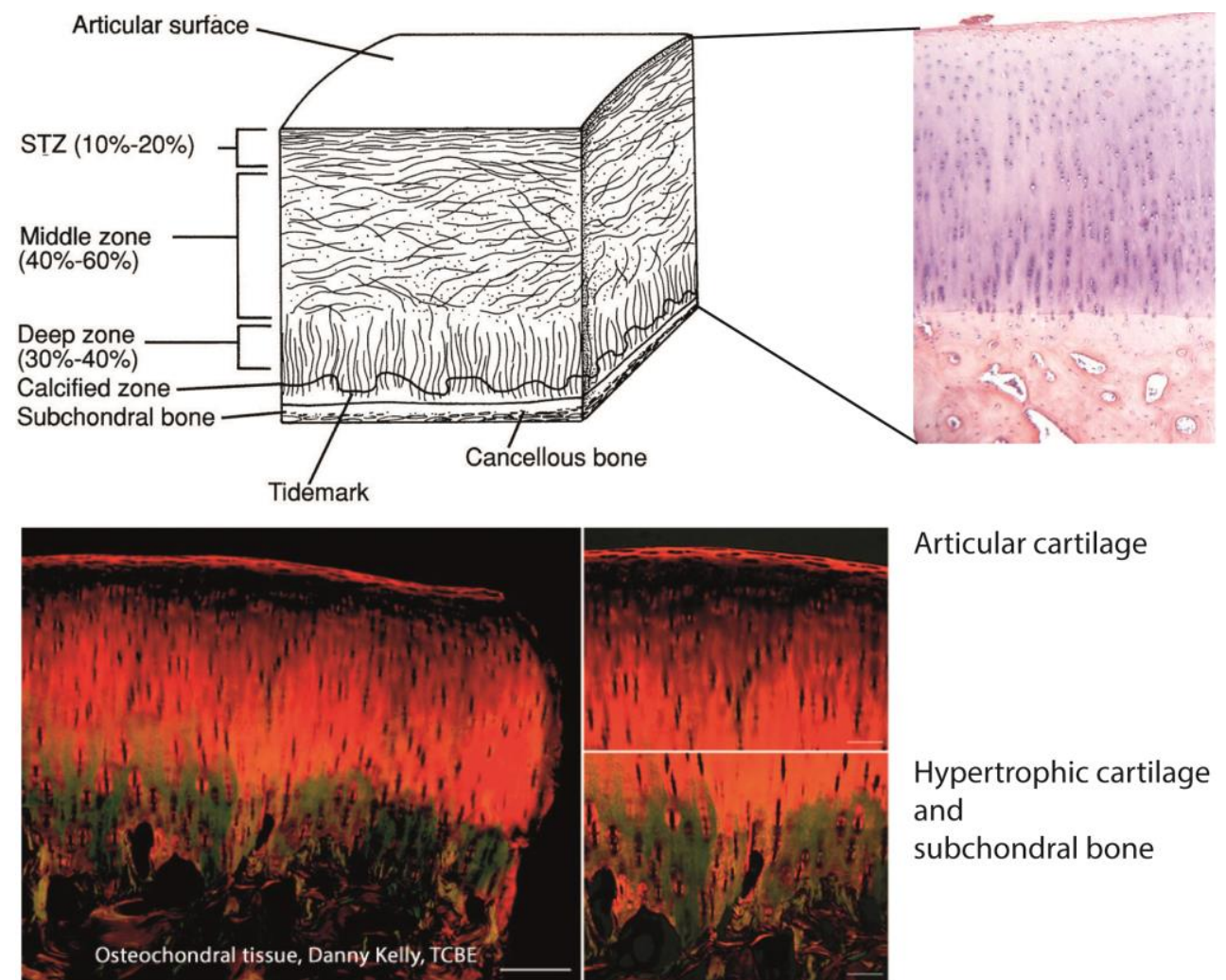

Articular cartilage

Hypertrophic cartilage and

subchondral bone

Figure 1. Schematic representation of the osteochondral tissue and its components and osteochondral tissue from two histological sections. Modified from Buckwalter, 1994, Kwan Tat , 2009, and https://www.tcd.ie/biosciences/gallery/.

\subsection{Subcondral bone and calcified cartilage}

Long bones are divided into diaphysis, being the central part of the bone, and the epiphysis, localized in proximity of the joints. The structure of bone in the two compartments differs by the radial variation of bone compactness. In the diaphysis, a zone of more compact bone, called cortical bone, can be identified in the external part. Cortical bone presents a porosity ranging from $5 \%$ to $30 \%$ and a bone volume fraction ranging from $85 \%$ to $90 \%$. Moving towards the center, a more porous structure, called cancellous or trabecular bone, is encountered. The porosity of trabecular bone ranges from $30 \%$ to $90 \%$ and its bone volume fraction from $5 \%$ to $60 \%$. Bone volume fraction decreases when moving towards the bone marrow 
channel, in which the space between the trabecula is wider and filled with the bone marrow. The subchondral bone in the epiphysis presents similar characteristics of the trabecular bone with bone volume fraction ranging from $6 \%$ to $36 \%$, a trabecular thickness of $100-190 \mu \mathrm{m}$, a trabecular concentration ranging from 0.61 trabecules $/ \mathrm{mm}$ to 2.06 trabecules $/ \mathrm{mm}$ and the space between them ranging from $320 \mu \mathrm{m}$ to $1670 \mu \mathrm{m}$ [41]. At the epiphysis the compact bone is reduced to a thin layer. The subchondral bone serves to keep the integrity of the overlying articular cartilage [42]. At the molecular and cellular level bone and cartilage significantly differ from each other. Bone extracellular matrix (ECM) is mainly based on type I collagen fibrils with mineral deposits of calcium and phosphate, called hydroxyapatite. Beside collagen, other structural proteins are present. Among them the most important are osteopontin, thrombospondin and bone sialoprotein for cell and hydroxyapatite attachment, and osteocalcin and osteonectin for the binding of hydroxyapatite and calcium respectively [43]. Being a vascularized tissue and due to the presence of the bone marrow, the cell composition of bone is very heterogeneous. Within the bone marrow, a small fraction $(0.002 \%)$ is represented by mesenchymal stromal/stem cells (hMSCs), which are the precursor, among others, of osteoblasts, osteoclast and chondrocytes. Osteoblasts are the cells responsible for hydroxyapatite synthesis and deposition. Osteoclasts have an opposite activity, being responsible of bone resorption. Beside their structural function, bones represent the reservoir of calcium for the human body. The activity of osteoblasts and osteoclasts is fine-tuned by hormones depending on the request of calcium from the body, which results in a process of bone synthesis and remodeling through the whole life of an individual [44]. The interplay between osteoblasts and osteoclasts is further controlled by osteocytes, the most abundant cell in bone tissue [45]. Besides being actively involved in maintaining the bony matrix, the osteocytes are also mechanotransductors. Their immersion in the bone matrix and connection with adjacent osteocytes in the canaliculi allows exogenous and endogenous signals to be transmitted via mechanical, electrical and chemical mechanisms [45]. Another cell type present in this tissue are the endothelial cells forming the blood vessels which run through the bones. Moving from the epiphysis toward the joint, the first portion of the cartilage encountered is the calcified zone. It 
is considered together with the subchondral bone, since it is a transition portion presenting some characteristics of the cartilaginous tissue, such as the deposition of collagen type $X$, and some characteristics of the bone tissue, such as the presence of alkaline phosphatase and of mineral deposits. Its function is to provide a good attachment between bone and cartilage, and transfer the forces from the joint to the subchondral bone [46].

\subsection{Articular cartilage}

The function of articular cartilage is to transfer and distribute the load forces to the subchondral bone; the disposition and localization of structural proteins such as collagen and proteoglycans is optimized to perform this function. Collagen type II, in particular, is the most abundant component of the ECM in hyaline cartilaginous tissue. Another abundant component of the ECM are the proteoglycans, responsible for the water uptake and osmolarity maintenance. There are two classes of proteoglycans, large aggregating proteoglycans such as aggrecan and smaller one such as decorin, biglycan and fibromodulin [47]. Articular cartilage can be divided into 3 main zones: moving from the articular surface to the subchondral bone, the superficial, the transition and the radial or deep zones. The superficial and transitional zones each constitute $10 \%$ of the height of the articular cartilage layer. The radial zone represents instead the bulk of articular cartilage, accounting for $80 \%$ of it [40]. Chondrocytes represent the $2 \%$ of the component of the articular cartilage and they are the only cell type present in this tissue. Chondrocytes are characterized by a rounded shape, different size and orientation along the cartilage height, and are surrounded by two main ECM proteins, namely collagen type II and aggrecan. Chondrocytes are fairly small cells, presenting a diameter of $13 \mu \mathrm{m}$, a surface of $821 \mu \mathrm{m}^{2}$ and a volume of $1748 \mu \mathrm{m}^{3}$, approximately. These features do not vary much among the cartilage zones with exception of the hypertrophic one [40]. When a chondrocyte becomes hypertrophic its volume increases up to 20 times [48] and starts to produce collagen X. Chondrocytes are organized in chondrons which are the primary structural, functional and metabolic unit in hyaline cartilage. A chondron comprises the chondrocyte and the pericellular molecular 
environment of which collagen type VI and IX are the major components. This ECM proteins are present only in close proximity of the cell, while they decrease at very low levels in the cartilage matrix. Beside an axial characterization of the matrix, another distinction can be done based on the matrix between one chondron and the following one. Close to the chondrocyte the pericellular matrix is rich in collagen type VI and IX, as just mentioned, with the addition of proteoglycans such as hyaluronans [49], sulfated proteoglycans [50] and biglycans [51] as well as matrix glycoproteins such as fibronectin [52], link protein [53] and laminin [54]. Moving away from the chondrocyte and its pericellular matrix, the territorial zone is encountered which is rich in chondroitin sulfate. Further away, in the space between chondrons, the inter-territorial matrix is localized in which the main proteoglycans are rich in keratin sulfate [53]. In the superficial and transitional zone, chondrons are exclusively single cells units, whereas in the radial zone they contain on average 5-8 chondrocytes [40].

Moving from the radial to the transitional zone the ECM maintains the same composition and presents decorin as proteoglycan in addition to aggrecan. In this region, collagen fibers pass from a vertical to a more horizontal orientation. The surface zone of articular cartilage is the one responsible to the lubrication of the joint. Its ECM is mainly composed by collagen type I [55], collagen type II and lubricin or PRG4, a proteoglycan present also in the synovial fluid responsible for joint lubrication and synovial homeostasis [56]. The collagen is responsible of the strength and the high shear stress resistance of the superficial layer [57]. Here, collagen fiber orientation progresses towards a completely horizontal configuration, which contributes to the low friction mechanical properties of hyaline cartilage. 

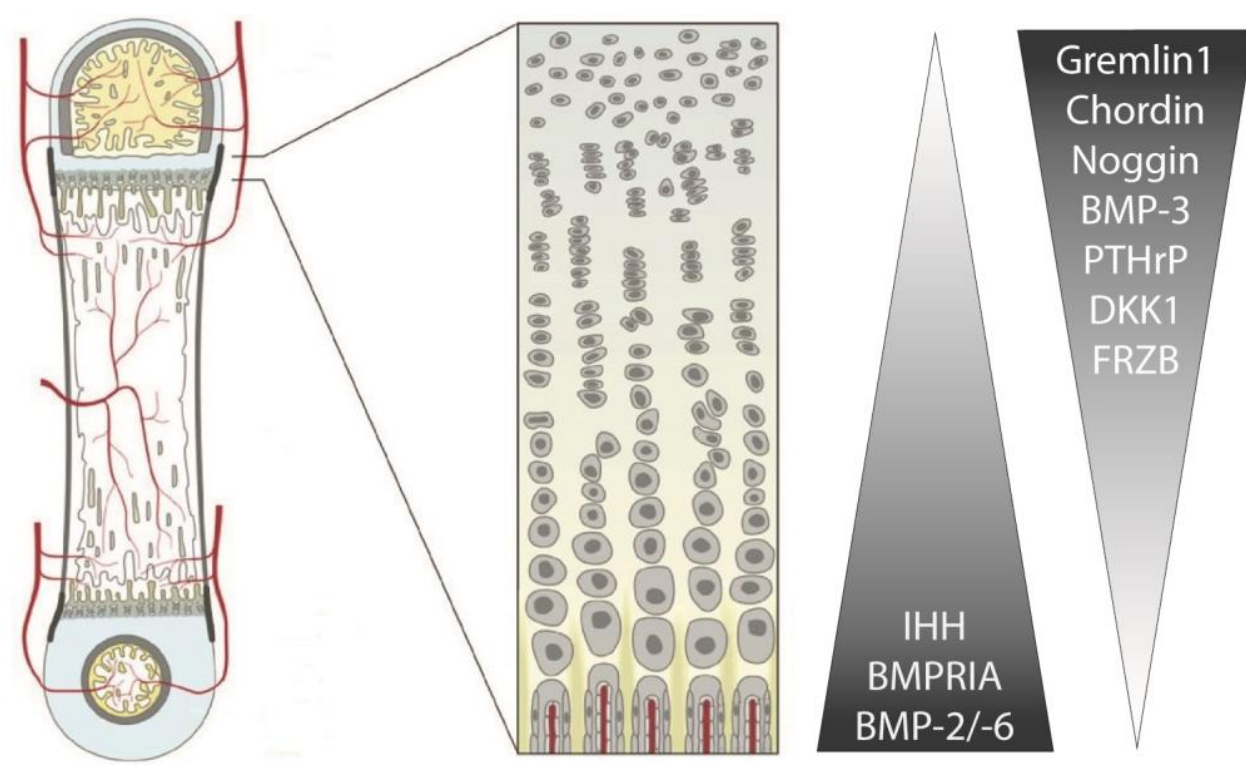

Figure 2. Schematic representation of the growth plate and the gradients formed by growth factors. The arrow direction describes a descendent gradient. Modified picture from http://serkadis.net/.

\section{The osteochondral tissue as a gradient tissue}

\subsection{Gradients in osteochondral development}

During development, a rich orchestration of growth factors act at different levels within the growth plate spatially and temporally (Fig. 2). To ensure the proliferation of progenitor cells on the superficial layer, but prevent it in the lower part, a PTHrP gradient is formed. "Elder cells" move downwards and keep dividing as long as the PTHrP effect is present. Once they reach the lower resting phase of the growth plate, the effect is no longer present and this make them proceeding toward the next step of maturation. Similarly, once the chondrocytes have reached the hypertrophic stage bone morphogenetic protein (BMP) signaling plays a major role in bone formation. The BMP effect is determined by a combination of cell susceptibility and BMP antagonist. BMP-2 and -6 are produced by osteoblasts and hypertrophic chondrocytes. Their expression is higher in the hypertrophic zones and decrease while moving upward in the resting and proliferative zone. Their 
effect is also decreasing from the hypertrophic zone toward the proliferation and resting zones since the expression of their receptor BMPRIA follows the same trend [58]. BMPs activity is finely regulated in order to stimulate the right portion of the growth plate, therefore an opposite gradient of BMPs antagonists can be also found. BMP-3 is considered an antagonist, since its action inhibits BMP stimulated bone formation [59]. Among the antagonists, noggin, gremlin, chordin and BMP-3 are more highly expressed in the resting zone compared to the proliferative and hypertrophic ones [58], thus resulting in an increased gradient of osteoinductive signals within the growth plate. The development of chondrocyte toward the hypertrophic state is finely tuned via the expression of antagonists such as Gremlin 1, Frizzled-Related Protein, and Dkk-1. Their expression presents a decreasing trend from the resting zone in which their presence is the highest toward the hypertrophic zone in which their levels are the lowest [31].

\subsection{Structural gradient - fibrils and ECM composition}

Due to its compartmental architecture, the $\mathrm{OC}$ tissue presents a variety of axial gradients along its structure. In the $\mathrm{OC}$ tissue, the structural gradient is defined by the composition and organization of the ECM (Fig. 1 and 3). As mentioned earlier, the subchondral bone is a mineralized tissue composed primarily by collagen type I and hydroxyapatite. Hydroxyapatite, the inorganic component of subchondral bone, comprises $85.8 \% \pm 3.4 \%$ of its dry weight, decreasing to $65.1 \% \pm 2.3 \%$ in the calcified cartilage to completely disappear in the hyaline cartilage [14]. Collagen type $I$ is present in bone and starts to decrease in content from the mineralized cartilage. In cartilage, it can be found only in the surface layer. In the mineralized cartilage lying above subchondral bone, the residing cells are a particular type of chondrocytes named hypertrophic chondrocyte, which present the peculiar characteristic of producing collagen type $\mathrm{X}$ and are embedded in mineralized ECM. In this deep zones of the $\mathrm{OC}$ tissue the direction of the fibrils do not play a major role, since the integrity and the shock absorption ability is given by the mineralization of the ECM and the presence of collagen type X. Collagen type II starts to appear in the radial zone in thick fibers, with a direction that is 
perpendicular to the articular surface. From the radial zone moving towards the superficial zone, mineralization also disappears. In the transitional zones collagen type II fibers increase in number, but decrease in diameter and assume a more parallel direction, to become totally parallel to the articular surface in the superficial zone. This direction determines the greatest tensile and shear strength [47]. Conversely, the amount of glycosaminoglycans (GAGs) forms a gradient opposite to the one generated by the collagen, decreasing from the radial zone toward the joint surface. GAGs are responsible for the gradient in stiffness within articular cartilage: as the total proteoglycan concentration decreases, the compressive stiffness also decreases [60].
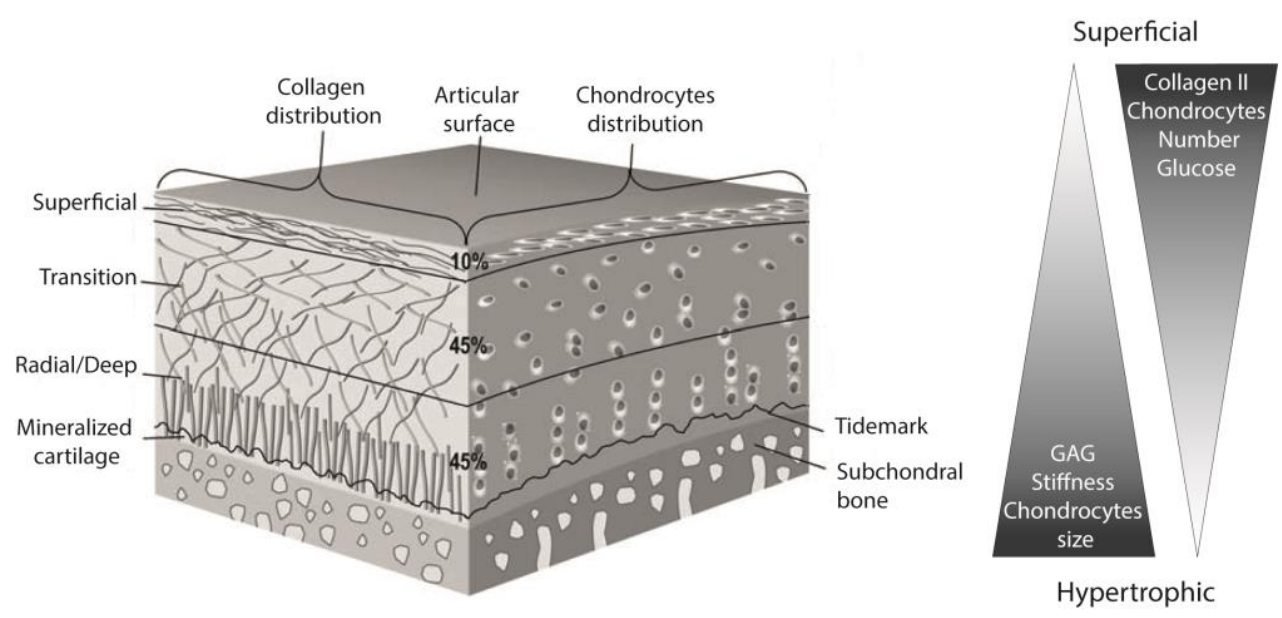

Figure 3. On the left side, schematic representation of the osteochondral tissue. On the right side, directions of its gradients. The stiffness gradient continue in the subchondral bone, whereas the nutrient gradient stops in the radial zone. Modified from Woodfield, 2005.

\subsection{Cellularity gradient and pericellular environment}

A variety of cells are present in the $O C$ tissue. As previously described, the subchondral bone presents osteoblasts for collagen and mineral deposition, osteoclasts for bone remodeling and calcium mobilization and osteocytes for their regulation and mechanotransduction [45]. Being highly vascularized, bone contains endothelial cells composing the vascular network, which are missing in articular cartilage due to its typical avascular nature. The variation from bone to cartilage 
tissue in terms of cells does not follow a gradient. On the other hand, within the cartilage tissue a gradient in cell distribution, size and deposited pericellular matrix can be observed. Within cartilage, the only cell type present has been always believed to be chondrocytes. Although defined as chondrocytes throughout the tissue, this simplification is misleading since profound differences are present in the behavior, morphology and function of these cells among the cartilage zones. Chondrocytes vary in number, size and disposition depending in which region they are located. The gradient in chondrocytes density per zone can be summarized by a variation from $7{ }^{\prime} 000$ to $24^{\prime} 000$ cells $/ \mathrm{mm}^{3}$ (Fig. 3). Beside the variation in cell number, chondrocyte appearance varies in a gradient way. The cell body decrease in size from the hypertrophic zone toward the superficial layer. Also the morphological and functional units of hyaline cartilage, the chondrons, display a gradient through the cartilage layers. In the radial zone, chondrons are arranged in columns. Even if chondrocytes have the characteristic of no direct cell-cell connections, the tail of the chondrons are entangled in order to guarantee continuity from one chondron to the following one. The number of chondrocytes per chondron is estimated to change from one to eight with the lowest number in the superficial zone and the highest in the radial zone [40,61]. Moving toward the articular surface, as the chondrocytes/chondron number decreases to one, the overall number of chondrocytes increases. Their appearance passes from column like structures to single entities dispersed in the ECM. An additional switch in cell number, function and morphology can be seen from the transition to the superficial region of the articular cartilage. The collagen produced by this cells change from collagen type II to collagen type I. Their morphology change from rounded to flat and they form a superficial cell layer with a very low amount of ECM separating them from each other. Additionally, the superficial region is the area with the highest proliferative rate $[40,55]$. From a developmental point of view, the maturation of chondrocytes follows an opposite trend compared to the cell density. In 2001, it has been determined by Hayes et al. that the development of chondrocytes follows an appositional direction [62]. The cartilage progenitor cells are located in the superficial region and their development is proven to pass through the cell phenotypes encountered from the superficial to the deep region. 
Eventually the chondrocyte would enter in the hypertrophic stage, which is thought to be the last stage of their development [26].

\subsection{Stiffness gradient}

From the subchondral bone, the mineralization and stiffness of the ECM decrease when moving toward the articular surface, as shown in Figure 3. This can be defined as a discrete gradient, since the subchondral bone and the mineralized cartilage present the highest amount of minerals that disappear completely in the other zones composing the articular cartilage. The subchondral bone has an elastic modulus of $3.9 \pm 1.5 \mathrm{GPa}$, which decreases in the mineralized cartilage to $0.32 \pm$ $0.25 \mathrm{GPa}$ [63]. From the mineralized cartilage region moving towards the articular surface, the stiffness decreases from $0.66 \pm 0.05 \mathrm{MPa}$ in the deep zone to $0.24 \pm$ $0.05 \mathrm{MPa}$ in the superficial zone [64]. Articular cartilage has also the peculiarity to change its mechanical properties when a loading is applied dynamically. Due to its highly viscoelastic character, its dynamic stiffness increases with increasing the loading frequency, spanning from $4 \mathrm{MPa}$ to $10 \mathrm{MPa}[65,66]$. The final load is borne by the bone and the cartilage has the function to transfer the forces through the ECM.

\subsection{Nutrients/02 gradient}

The bone side of the $\mathrm{OC}$ tissue is reached by blood vessels that are responsible for bone nutrient supply and waste removal. One of the major anatomical characteristics of cartilage, instead, is the absence of blood vessels through the entire width. Nutrients are transported to the cells mainly by diffusion from the synovial fluid. Nutrient transport is also sometimes thought to be assisted by movement of fluid in and out of cartilage in response to cyclic loading of the tissue [67]. Therefore, gradients of nutrients and metabolic byproducts exist through cartilage due to the balance between transport and rates of cellular metabolism [68]. This process is aided by the presence of proteoglycans that have the ability of retaining water, which flows in with the nutrients during loading relaxation and flows out with the metabolites during compression. Among the nutrients, glucose 
represents the primary source of energy. Pyruvate, the product of glycolysis, is converted in acetil coenzyme $\mathrm{A}$, which is the main supplier of carbon in Krebs cycle. A glucose gradient from the synovial fluid toward the deep zone of cartilage is also present as shown by Zhou et al. [68]. Being glucose not only the most important source for energy production, but also a key component in GAG synthesis, it has been shown that across cartilage from the synovial side to the subchondral bone, a glucose gradient exists [69] (Fig. 3). Similarly to glucose, the oxygenation of cartilage comes from the synovial fluid. Therefore, a gradient of oxygen is formed from the joint of the surface toward the radial zone (Fig. 3). The oxygen tension of synovial fluid in humans is $6.5-9.0 \%(50-70 \mathrm{~mm} \mathrm{Hg})[70,71]$. Measured oxygen tension in articular cartilage ranges from $7 \%(53 \mathrm{~mm} \mathrm{Hg})$ in the superficial layer to less than $1 \%(7.6 \mathrm{~mm} \mathrm{Hg})$ in the deep zone $[72,73]$ of the oxygen tension of synovial fluid.

\section{Gradient scaffolds for osteochondral regeneration}

\subsection{General requirements}

In the past few years the number publications involving scaffolds addressing the gradient nature of the $\mathrm{OC}$ tissue has greatly increased. Studies involving structural porosity gradient were proved to increase the cell seeding efficiency of human osteosarcoma cell line SaOs-2 [74] and to mimic in vitro the gradient structure of the cartilage in terms of chondrocytes and ECM distribution [75]. In literature it is possible to find several studies aiming at the treatment of articular cartilage alone or at the development of biphasic scaffolds for the treatment of bone and cartilage as separate tissues. Due to the wide range of gradients present in the osteochondral tissue, several choices can be made in terms of gradient scaffolds. Typically, bi-phasic or tri-phasic scaffolds have been developed, displaying either discrete or continuous gradients [76-79]. In order to define bi- or tri-phasic scaffolds different materials can be chosen. In this respect, the selection of biomaterials needs to take into account not only their biocompatibility, but also the effect that each material will play on the seeded cells. For the scaffold preparation, natural or 
synthetic polymers can be used. A logical criteria for the selection of natural polymer candidates passes through the components of the ECM. For the bone compartment, collagen type I combined with hydroxyapatite (HA) were used to produce scaffolds or to cover the underlying polymer by coating the pore surface [11], in order to mask the synthetic polymer and present an ECM like environment for cell attachment, growth and differentiation. For the regeneration of the cartilage portion, collagen type II, glycosaminoglycans, and hyaluronic acid have been used [80]. For both bone and hyaline cartilage, the most common synthetic polymers used are poly lactic acid (PLLA) [81], poly glycolic acid (PGA) [11] and polycaprolactone (PCL) [82].

Among other properties, an osteochondral scaffold should be biodegradable with a degradation rate matching the rate of ECM production, and should have high levels of porosity and pore interconnectivity for cell attachment, and gas, nutrient, and waste products exchange, [11]. The mechanical properties of the fabricated scaffolds should match the gradient in mechanical properties present in the osteochondral tissue, with a stiffer scaffold for the bone compartment and a gradually softer scaffold for the cartilage compartment. Additionally, the inner geometry of the construct must be taken into consideration, ideally mimicking the structural and architectural properties of the native tissue.

\begin{tabular}{|c|c|c|c|c|}
\hline Author & $\begin{array}{c}\text { In } \\
\text { vitro/in } \\
\text { vivo }\end{array}$ & $\begin{array}{c}\text { Material } \\
\text { Subchondral } \\
\text { bone + cells }\end{array}$ & $\begin{array}{c}\text { Material } \\
\text { Cartilage + cells }\end{array}$ & Main findings \\
\hline $\begin{array}{c}\text { Schaefer } \\
\text { et al. [4] }\end{array}$ & In vitro & $\begin{array}{c}\text { PGA non-woven } \\
\text { meshes; } \\
\text { chondrocytes }\end{array}$ & $\begin{array}{c}\text { PLGA/PEG } \\
\text { foams, } \\
\text { Periosteal cells }\end{array}$ & $\begin{array}{c}\text { Increased ECM } \\
\text { mineralization, } \\
\text { GAG synthesis, } \\
\text { integration with } \\
\text { subchondral } \\
\text { bone }\end{array}$ \\
\hline $\begin{array}{c}\text { Schek et } \\
\text { al. [6] }\end{array}$ & $\begin{array}{c}\text { In vivo, } \\
\text { nude }\end{array}$ & $\begin{array}{c}\text { HA ceramic } \\
\text { Fibroblasts } \\
\text { expressing BMP-7 }\end{array}$ & $\begin{array}{c}\text { PLLA sponge } \\
\text { Chondrocytes }\end{array}$ & $\begin{array}{c}\text { Bone and blood } \\
\text { vessel, } \\
\text { mineralized } \\
\text { transition tissue, } \\
\text { Cartilaginous } \\
\text { tissue }\end{array}$ \\
\hline $\begin{array}{c}\text { Cao et al. } \\
\text { [83] }\end{array}$ & In vitro & $\begin{array}{c}\text { Plotted PCL } \\
\text { Stromal cells from }\end{array}$ & $\begin{array}{c}\text { Plotted PCL } \\
\text { Rib cartilage }\end{array}$ & $\begin{array}{c}\text { Bone like tissue } \\
\text { formation }\end{array}$ \\
\hline
\end{tabular}




\begin{tabular}{|c|c|c|c|c|}
\hline & & iliac crest & chondrocytes & $\begin{array}{c}\text { Cartilage-like } \\
\text { ECM }\end{array}$ \\
\hline $\begin{array}{l}\text { Ding et al. } \\
\text { [82] }\end{array}$ & $\begin{array}{l}\text { In vivo, } \\
\text { nude } \\
\text { mice }\end{array}$ & $\begin{array}{c}\text { Plotted PCL + HA } \\
+ \text { MSC }\end{array}$ & $\begin{array}{l}\text { PGA/PLA non- } \\
\text { woven fibers + } \\
\text { chondrocytes }\end{array}$ & $\begin{array}{l}\text { Osteogenic and } \\
\text { chondrogenic } \\
\text { markers, } \\
\text { interface with } \\
\text { hypertrophic } \\
\text { cartilage }\end{array}$ \\
\hline $\begin{array}{l}\text { Kandel et } \\
\text { al. [84] }\end{array}$ & $\begin{array}{l}\text { In vivo, } \\
\text { sheep }\end{array}$ & CPP & $\begin{array}{l}\text { Chondrocyte } \\
\text { layer }\end{array}$ & $\begin{array}{l}\text { Osteochondral } \\
\text { regeneration, } \\
\text { some sign of } \\
\text { cartilage } \\
\text { maturation }\end{array}$ \\
\hline $\begin{array}{c}\text { Duan et al. } \\
\text { [85] }\end{array}$ & $\begin{array}{l}\text { In vivo, } \\
\text { rabbit }\end{array}$ & $\begin{array}{c}\text { Salt leached } \\
\text { PLGA 300-450 } \\
\mu \mathrm{m}+\mathrm{MSC}\end{array}$ & $\begin{array}{c}\text { Salt leached } \\
\text { PLGA 100-200 } \\
\mu \mathrm{m}+\mathrm{MSC}\end{array}$ & $\begin{array}{l}\text { simultaneous } \\
\text { regeneration of } \\
\text { articular } \\
\text { cartilage } \\
\text { and subchondral } \\
\text { bone } \\
\end{array}$ \\
\hline $\begin{array}{l}\text { Chen et al. } \\
\text { [86] }\end{array}$ & $\begin{array}{l}\text { In vivo, } \\
\text { rabbit }\end{array}$ & $\begin{array}{l}\text { BMP-2-activated } \\
\text { HA/chitosan- } \\
\text { gelatin }\end{array}$ & $\begin{array}{c}\text { TGF- } \beta 1- \\
\text { activated } \\
\text { chitosan-gelatin }\end{array}$ & $\begin{array}{l}\text { regeneration of } \\
\text { articular } \\
\text { cartilage and } \\
\text { subchondral } \\
\text { bone }\end{array}$ \\
\hline $\begin{array}{l}\text { Re'em et } \\
\text { al. [87] }\end{array}$ & $\begin{array}{l}\text { In vivo, } \\
\text { rabbit }\end{array}$ & $\begin{array}{c}\text { alginate-sulfate, } \\
\text { alginate, } 0.18 \% \text { - } \\
\text { gluconic } \\
\text { acid/hemicalcium } \\
\text { salt + BMP4 }\end{array}$ & $\begin{array}{c}\text { alginate-sulfate, } \\
\text { alginate, } \\
0.18 \% D \text {-gluconic } \\
\text { acid/hemicalcium } \\
\text { salt + TGF- } \beta 1\end{array}$ & $\begin{array}{c}\text { ECM } \\
\text { composition of } \\
\text { hyaline cartilage } \\
\text { with no signs of } \\
\text { mineralization, } \\
\text { new formed } \\
\text { woven bone in } \\
\text { the bottom part. }\end{array}$ \\
\hline
\end{tabular}

Table 1. Summary of the biphasic scaffolds described, indicating the biomaterials, cells and growth factors used and the main results for each construct.

\subsection{Choosing cells}

After considering general design criteria in fabricating a scaffold for osteochondral regeneration, one should consider whether the scaffold will be directly implanted or seeded with cells before implantation. In the latter case, two main solution have been proposed: (i) the direct use of already differentiated cells $[5,83,88]$ or (ii) the use of progenitor cells, namely hMSCs from different sources [8, 89]. When differentiated cells are used, the part of the scaffold which will be in contact with the subchondral bone is loaded with osteoblasts; in order to allow vascularization, this 
portion of the scaffolds must provide the space for vessel ingrowth. Therefore, an interconnected pore network is required. For the portion of the scaffold aimed to regenerate hyaline cartilage, the use of chondrocytes is a natural choice. Being cartilage not vascularized, the structure of the scaffold doesn't need as large pores as the ones in the bone side. The use of already differentiated cells presents some limitations, since cells must be harvested, expanded in laboratory, seeded in the construct and finally implanted. Another possibility is the seeding of progenitor cells [55]. Since both chondrocytes and osteoblast originates from the bone marrow, the use of bone marrow derived hMSCs has attracted a lot of interest for tissue engineering applications, either when these cells are used alone or in combination with scaffolds [90-92]. When hMSCs are used, the concept of scaffold design changes since it must present the right cues to direct their differentiation toward the osteogenic and chondrogenic lineage in the proper compartment of the construct. Several studies are aiming at generating scaffolds able to drive hMSC differentiation toward the targeted lineage. Among them, the use of growth factors in soluble form or bound to the scaffold structure are the ones presenting the strongest effects $[8,16,93]$. Recent studies demonstrated that hMSCs respond to stimuli coming from the matrix stiffness as well [94, 95]. Cells attaching to a soft material are more prone to differentiate toward the chondrogenic lineage, whereas cells on a stiffer material will be driven toward the osteogenic lineage. Yet, the use of hMSCs in the context of classical tissue engineering, where cells are expended and then seeded in vitro to allow for tissue formation before constructs can be implanted, presents some limitation such as the time of culture and the need of external factors for their differentiation in vitro. A further approach to overcome this problem may consist in the development of in vitro systems that drive the differentiation of hMSCs in a regional way, depending on the part of the osteochondral tissue that they will be in contact with. Once such a system is developed and validated with in vitro and in vivo studies, one could consider to directly implant this scaffold and exploit the intrinsic presence of hMSCs in the implantation site. When combined with surgical techniques like microfracture, where small holes are drilled to reach the bone marrow present in the subchondral bone compartment, the bone marrow will flow in the scaffold from the underlying 
bone providing the hMSCs that will colonize the scaffold and differentiate toward the right lineage depending on the cues provided by the construct.

\begin{tabular}{|c|c|c|c|c|c|}
\hline Author & $\begin{array}{l}\text { In } \\
\text { vitro/in } \\
\text { vivo }\end{array}$ & $\begin{array}{c}\text { Material } \\
\text { Subchondr } \\
\text { al bone + } \\
\text { cells }\end{array}$ & $\begin{array}{l}\text { Intermediate } \\
\text { zone material } \\
\text { + cells }\end{array}$ & $\begin{array}{l}\text { Material } \\
\text { Cartilage + } \\
\text { cells }\end{array}$ & Main findings \\
\hline $\begin{array}{l}\text { Sherwood } \\
\text { et al. [96] }\end{array}$ & In vitro & $\begin{array}{c}\text { L-PLGA } \\
(85: 15) / T C \\
\text { P with 55\% } \\
\text { porosity }\end{array}$ & $\begin{array}{l}\text { Increment in } \\
\text { porosity and } \\
\text { PLLA } \\
\text { content }\end{array}$ & $\begin{array}{c}\text { Salt leached } \\
\text { D,L-PLGA } \\
\text { (50:50)/L- } \\
\text { PLA with } \\
90 \% \\
\text { porosity + } \\
\text { chondrocyte } \\
\text { s }\end{array}$ & $\begin{array}{c}\text { Composite } \\
\text { tensile } \\
\text { strenght } \\
\text { similar to } \\
\text { cancellous } \\
\text { bone, } \\
\text { cartilage like } \\
\text { tissue } \\
\end{array}$ \\
\hline $\begin{array}{l}\text { Jiang et } \\
\text { al. [97] }\end{array}$ & In vitro & $\begin{array}{c}\text { PLGA } \\
\text { microspher } \\
\text { es }+ \\
\text { bioglass } \\
\text { seeded } \\
\text { with } \\
\text { osteoblasts }\end{array}$ & $\begin{array}{c}\text { Chondrocyte } \\
\text { s embedded } \\
\text { in an } \\
\text { agarose } \\
\text { hydrogel } \\
\text { + composite } \\
\text { microsphere } \\
\text { s } \\
\end{array}$ & $\begin{array}{c}\text { Chondrocyte } \\
\text { s embedded } \\
\text { in an } \\
\text { agarose } \\
\text { hydrogel }\end{array}$ & $\begin{array}{c}\text { Proper tissue } \\
\text { in each zone, } \\
\text { good } \\
\text { stratification }\end{array}$ \\
\hline $\begin{array}{l}\text { Heymer } \\
\text { et al. [98] }\end{array}$ & In vitro & $\begin{array}{c}\text { PLA + HA } \\
+\beta T C P\end{array}$ & $\begin{array}{l}\text { Hydrophobic } \\
\text { phase from } \\
\text { the } \\
\text { combination } \\
\text { of the two } \\
\text { phases }\end{array}$ & $\begin{array}{l}\text { Coll I + } \\
\text { hyaluronan } \\
+ \text { hMSCs }\end{array}$ & $\begin{array}{l}\text { Cartilage like } \\
\text { cells, coll II, } \\
\text { proteoglycans, } \\
\text { cartilage } \\
\text { specific } \\
\text { markers gene }\end{array}$ \\
\hline $\begin{array}{l}\text { Levingsto } \\
\mathrm{n} \text { et al. } \\
\text { [9] }\end{array}$ & In vitro & $\begin{array}{c}\text { collagen } \\
\text { type I and } \\
\text { HA + } \\
\text { MC3T3 }\end{array}$ & $\begin{array}{l}\text { collagen } \\
\text { type I, } \\
\text { collagen } \\
\text { type II and } \\
\text { HA + } \\
\text { MC3T3 }\end{array}$ & $\begin{array}{c}\text { collagen } \\
\text { type I and II, } \\
\text { hyaluronic } \\
\text { acid and } \mathrm{Na} \\
\text { salts }+ \\
\text { MC3T3 } \\
\end{array}$ & $\begin{array}{c}\text { Biocompatibilit } \\
\text { y of the } \\
\text { construct }\end{array}$ \\
\hline $\begin{array}{l}\text { Marquass } \\
\text { et al. [99] }\end{array}$ & $\begin{array}{l}\text { In vivo, } \\
\text { sheep }\end{array}$ & TCP & $\begin{array}{l}\text { activated } \\
\text { plasma gel }\end{array}$ & $\begin{array}{c}\text { collagen } \\
\text { type I } \\
\text { hydrogel }\end{array}$ & $\begin{array}{c}12 \text { months } \\
\text { repair } \\
\text { comparable to } \\
\text { autograft }\end{array}$ \\
\hline $\begin{array}{c}\text { Liu et al. } \\
\text { [100] }\end{array}$ & $\begin{array}{l}\text { In vivo, } \\
\text { rabbit }\end{array}$ & $\begin{array}{l}\text { TCP + } \\
\text { MSCs }\end{array}$ & & $\begin{array}{c}\text { collagen I } \\
\text { and } \\
\text { hyaluronan } \\
+ \\
\text { electrospun } \\
\text { PCL mesh + } \\
\end{array}$ & $\begin{array}{c}\text { improved } \\
\text { regeneration } \\
\text { histological } \\
\text { scores } \\
\text { matched } \\
\text { autografts } \\
\end{array}$ \\
\hline
\end{tabular}




\begin{tabular}{|c|c|c|c|c|c|}
\hline & & & & MSCs & \\
\hline $\begin{array}{c}\text { Dresing } \\
\text { et al. } \\
\text { [101] }\end{array}$ & $\begin{array}{l}\text { In vivo, } \\
\text { rabbit }\end{array}$ & $\begin{array}{c}\text { salt } \\
\text { leached } \\
\text { PUR and } \\
\text { nano HA }\end{array}$ & $\begin{array}{c}\text { Electrospun } \\
\text { PUR }\end{array}$ & $\begin{array}{c}\text { Salt leached } \\
\text { PUR }\end{array}$ & $\begin{array}{l}\text { no advantage } \\
\text { in tissue } \\
\text { healing }\end{array}$ \\
\hline $\begin{array}{l}\text { Jeon et } \\
\text { al. [88] }\end{array}$ & $\begin{array}{l}\text { In vivo, } \\
\text { Nude } \\
\text { rat/cow }\end{array}$ & $\begin{array}{c}\text { FDM } \\
\text { plotted } \\
\text { PCL }+ \\
\text { osteoblasts } \\
+ \text { rhBMP-7 }\end{array}$ & $\begin{array}{l}\text { electrospun } \\
\text { PCL layer + } \\
\text { osteoblasts } \\
+ \text { rhBMP-7 }\end{array}$ & $\begin{array}{c}2 \% \text { alginate } \\
+ \text { superficial } \\
\text { chondrocyte } \\
\text { on the upper } \\
\text { part and } \\
\text { middle-deep } \\
\text { chondrocyte } \\
\text { in the lower } \\
\text { zone }\end{array}$ & $\begin{array}{l}\text { No cartilage } \\
\text { compartiment } \\
\text { al structure, } \\
\text { no bone } \\
\text { ingrowth and } \\
\text { mineralization }\end{array}$ \\
\hline
\end{tabular}

Table 2. Summary of the triphasic scaffolds described, indicating the biomaterials, cells and growth factors used and the main results for each construct.

\subsection{Biphasic constructs}

In its most simplistic version the osteochondral tissue can be seen as a bi-phasic tissue being composed by a bone phase and a cartilaginous phase. In most studies, biphasic scaffolds are not only based on the different materials or structures of the two components, but also on the different cells cultured in the resulting scaffolds. In 2000, the work of Schaefer et al. presented an in vitro engineered scaffold based on PGA non-woven meshes seeded with primary bovine chondrocytes and PLGA/PEG foams seeded with expanded periosteal cells. The two constructs showed promising results in vitro, due to an increase in GAG production in the cartilage side, an increase in mineralization of the ECM in the bone side, and a good integration of the two phases after 4 weeks of culture [4]. Similarly, in the work of Schek et al., the constructs presented a biphasic design in material, cell types and growth factors. The scaffold consisted in the combination of a ceramic based on HA on the bone side and a PLLA sponge on the cartilage side. The two parts were seeded with fibroblasts expressing BMP-7 on the ceramic side and chondrocytes within the sponge. The implantation of these constructs in nude mice determined the generation of a bi-phasic tissue with all the structures present in the osteochondral interface, bone and blood vessel in the ceramic part, a mineralized transition tissue, and a cartilaginous tissue on the polymeric side [6]. 
These examples showed initial promising results in achieving OC tissue regeneration. Yet, the scaffold structures developed in these studies are still characterized by a tortuous and not completely interconnected pore network, thus limiting their potential translation to larger defects where nutrient diffusion and waste removal become more critical for the viability and functionality of the regenerated tissue. For these reasons, additive manufacturing technologies gathered a great interest and momentum in the past two decades, due to their versatility in tuning scaffold features such as fiber spacing, fiber diameter and fiber deposition pattern. This allows to obtain fully interconnected 3D structures with customizable pore network, resulting in a high flexibility in tuning physico-chemical, structural, and mechanical properties of the fabricated scaffolds. Cao et al. [83] studied the co-culture of iliac crest stromal cells and chondrocyte in a bi-phasic PCL construct. The scaffold was plotted and then partitioned vertically into two halves with a gap between them, leaving only a small portion in contact with each other. The first half was seeded with iliac crest stromal cells and cultured for 18 days under osteogenic conditions, the other half was seeded with rib cartilage chondrocytes. The resulting co-culture construct was cultured in vitro for 8 weeks in a co-culture medium. The two compartment were kept together by the addition of fibrin glue to both cell suspensions prior to seeding. Even though the in vitro results were satisfactory, additional analysis before further use of this scaffold in vivo should be performed as also suggested by the authors. More recently, Ding et al. [82] tested a biphasic scaffold to regenerate a goat femoral head. This in vivo study was carried in nude mice and the results were analyzed by using goat proximal femoral condyles as positive controls and implanted cell-free scaffolds as negative controls. The scaffold had a femoral condyle shape and consisted in a lower part made of a blend of PCL and HA plotted via fused deposition modeling, on top of which a non-woven fiber scaffolds of PGA/PLA was located to regenerate the articular cartilage tissue of the femoral head. The two components of the scaffolds were seeded with goat hMSCs in the bone compartment and chondrocytes in the cartilage compartment, respectively. The cell seeded constructs were combined after 2-3 weeks of culture under osteogenic medium for the lower part and chondrogenic medium for the top part. The combined scaffolds were implanted and 
analyzed after 10 weeks, showing expression of osteogenic and chondrogenic markers in the two components, whereas the negative controls presented only sparse fibrotic tissue. Furthermore, this system generated an interface zone in which hypertrophic cartilage with immature calcified tissue could be observed, thus approaching the structural and biophysical properties of the native tissue.

To test scaffolds for OC regeneration in vivo the use of mice or rats is limitative, allowing only subcutaneous implantations. Larger animal models such as rabbits, pigs, or goats are necessary in order to fully validate new scaffolds in an orthotopic implantation model. Kandel et al. [84] produced a porous calcium poly-phosphate (CPP) scaffold seeded with autologous chondrocyte and implanted the construct in a sheep osteochondral defect. The scaffolds supported osteochondral regeneration with shown indications of cartilage maturation, bone ingrowth and fusion proceeding from 4 to 9 months of implantation. Yet, some fibrotic tissue was also observed at the bone interface, thus indicating a sub-optimal integration of the construct. Duan et al. [85] tested biphasic scaffolds with different pore sizes in combination with and without hMSCs in a rabbit model for osteochondral regeneration. The scaffolds were prepared by PLGA salt leaching technique and presented a top part for the chondral regeneration with an height of $1 \mathrm{~mm}$ and an osseous layer of $4 \mathrm{~mm}$. The constructs were implanted for 12 weeks. The scaffolds without cells displayed a better results than the untreated defects, defects implanted with cell-free scaffolds showed inferior repair in the chondral layer. Among the 5 pore size combinations tested, the construct with best histological and biochemical scores had pore size of $100-200 \mu \mathrm{m}$ for the chondral side, and 300 450 for the osseous part in combination with cells.

The use of autologous already differentiated cells has some limitations in terms of source and harvesting procedures. Thus, to overcome these drawbacks and to mimic what happens in the healing process of bone and cartilage, research efforts moved to the use of MSCs. In order to direct their differentiation toward the chondrogenic and the osteogenic lineage, the design of scaffolds geared to further functionalization to present growth factors in the right construct location in space and time. The combination of hMSCs seeded scaffolds and delivery of biological signals was studied in vivo by Chen et al. [86]. The constructs consisted of a 
plasmid TGF- $\beta 1$-activated chitosan-gelatin scaffold for the chondrogenic layer and a plasmid BMP-2-activated HA/chitosan-gelatin scaffold for the osteogenic layer. The structures were separately seeded with hMSCs and cultured for 1 week. Prior to implantation, the separated constructs were fused with fibrin glue and cultured for another week in vitro. The constructs promoted the growth and differentiation of hMSCs and supported the regeneration of articular cartilage and subchondral bone in a rabbit knee defect after 12 weeks of implantation. The same outcome was reached one year later by Re'em et al. [102], with a cell-free scaffold based on $0.1 \%$ alginate-sulfate $(w / v), 1 \%$ alginate $(w / v)$ and $0.18 \%(w / v)$ D-gluconic acid/hemicalcium salt loaded with TGF- $\beta 1$ and BMP-4 prepared in situ. After 4 weeks of implantation in rabbits, the top layer was integrated with the surrounding cartilage and displayed the typical ECM composition of hyaline cartilage with no signs of mineralization. The bottom part presented new formed woven bone.

The use of bi-phasic scaffolds has shown some degree of success, therefore highlighting that the strategy to mimic as closely as possible the native $\mathrm{OC}$ tissue is a promising route for functional regeneration. However, when strictly looking at biomimicry principles bi-phasic scaffolds are not satisfactory since they treat bone and cartilage as two distinct compartments, whereas the two tissues are intimately interconnected, as previously explained. Additionally, in most of the above presented studies the presence of only two parts implicitly neglected the presence of the transition zone of calcified cartilage. Furthermore, bi-phasic scaffolds do not display all the gradients that characterize the osteochondral tissue and the further division of cartilage into its specific zones.

\subsection{Tri-phasic and multi-layered scaffolds}

In the effort to mimic more closely the native osteochondral tissue, tri-phasic or multi-layered scaffolds have been developed by several groups. Two studies presented an intriguing scaffold design but their biological relevance was not studied in vitro nor in vivo $[11,103]$. In the first case a gradient in mechanical properties was generated. In the second study, only natural materials from the osteochondral ECM were used and an interface similar in ECM properties to the 
natural $\mathrm{OC}$ tissue interface was generated via the phenomena of interdiffusion of the two material phases followed by freeze drying. The bone side of the triphasic scaffolds is the most consistent since the major part of the studies found in literature presented a composite of PLGA or PLA combined with tricalcium phosphate, bioglasses or HA. On the other hand the cartilaginous portion varied in composition, material used and production method. In 2002 Sherwood et al. [96] presented multiphasic scaffolds. Each phase consisted of a different porosity, material composition and stiffness. The cartilage compartment was made by D,LPLGA (50:50)/L-PLA with $90 \%$ porosity obtained by salt leaching. The bone compartment was a composite made by a L-PLGA (85:15)/tricalcium phosphate with a porosity of $55 \%$. In order to prevent delamination, the intermediate area presented an increase in porosity (from $65 \%$ to $85 \%$ ) as well as in lactic acid content (from PLGA 85:15 to PLGA 50:50 PLA) when moving from the bone to the cartilage compartment. The mechanical testing proved that the composite had a tensile strength similar to cancellous bone. Chondrocyte were seeded, preferably attached to the cartilage scaffold compartment and after 6 weeks of culture in vitro formed cartilage like tissue. By solving the problem of delamination, however, the resemblance of the construct to $\mathrm{OC}$ tissue was compromised. A better result in terms of tissue stratification within the construct was obtained by Jiang et al. [97]. In this study, the variation in phases was generated by different combinations of materials and cells. Similarly to the work of Sherwood, the bone compartment was based on PLGA microspheres with a mineral part consisting of bioglass seeded with osteoblasts. Chondrocytes embedded in an agarose hydrogel formed the cartilage compartment. The intermediate phase was similar to the cartilage one with the addition of PLGA and bioglass ceramic microspheres, in order to stimulate the formation of mineralized cartilage. In each region, cells formed the proper tissue. Even if in the intermediate zone no markers of hypertrophic cartilage were analyzed, chondrocyte within a mineralized matrix were observed. The stratification of tissues within the construct was satisfactory. However, hydrogels lack sufficient mechanical properties, which are in the order of magnitude of tens to hundreds $\mathrm{kPa}$ whereas cartilage and subchondral bone mechanical properties are in the order of 0.45-0.8 MPa [60] and 2.3 $\pm 1.5 \mathrm{GPa}$ [63], respectively. Beside the similarities in 
the bone compartment Heymer et al. [89] used a different approach for the design of the chondral side. A porous layer of $3 \mathrm{~mm}$ of bovine collagen type I and hyaluronan composed the cartilage portion, while the bone side was based on PLA composite with a mineral phase of HA and $\beta$-tricalcium phosphate. In between the two layer, an hydrophobic region resulted from joining the two structures. The study focused on cartilage regeneration only. hMSCs were suspended in a collagen type I gel and seeded on the upper layer. After 3 weeks of culture under chondrogenic conditions, chondrocyte-like cells were visible in the upper third of the top layer and were surrounded by collagen type II and proteoglycans. At a molecular level, cells expressed specific marker genes such as COMP, aggrecan, collagen type II and X. Despite promising results for cartilage regeneration, the lack of bone regeneration results in only a partial validation of such a scaffold design. Recently, Levingston et al. [9] developed a system to generate gradients in material composition, stiffness and porosity via an "iterative layering" freeze-drying technique, based on the consequent apposition of layers on top of each other followed by freeze-drying. This design differed from the previous one as the scaffold composition was based only on natural polymers commonly found in the osteochondral ECM. The bone layer was composed by a commercial form of collagen type I and HA. The intermediate layer was produced by a mixture of collagen type I, collagen type II and HA. Similarly, the cartilaginous layer was based on collagen type I and II, hyaluronic acid and sodium salts. The scaffolds were seeded with MC-3T3 preosteoblasts, which proliferated and populated all the compartments over a period of 14 days confirming the biocompatibility of the construct. Such constructs may be promising for osteochondral applications, although an in vitro study with human cells and validation in preclinical animal models are needed to further confirm these preliminary results.

Three-layered constructs were also tested in vivo in large animal models. In 2010 Marquass et al. [99] compared osteochondral autografts with scaffolds presenting a collagen type I hydrogel on the chondral side, an intermediate activated plasma gel phase and a tricalcium phosphate osseous phase. The constructs were seeded with pre-differentiated autologous MSCs toward the chondrogenic lineage and implanted for 6 and 12 months in an osteochondral defect in sheep knee. No major 
differences in terms of histological scores were displayed by the groups. At 12 months biomechanical and macroscopic analysis didn't show any difference. Autologous MSC-seeded triphasic implants showed comparable repair quality to osteochondral autografts in terms of histology and biomechanical testing. More recently, multilayered scaffolds based on synthetic polymers such as PCL and polyurethane, with and without the addition of natural ECM components were evaluated in vivo by Liu et al. [100] and by Dresing et al. [101]. In both studies MSCs were seeded and the scaffolds implanted in a rabbit model for 12 weeks. Starting from an electrospun PCL mesh as periosteal scaffold, Liu et al. freeze dried it first with a collagen I and hyaluronan solution in order to generate the cartilage portion, and then with tri-calcium phosphate for the osseous phase. The electrospun mesh promoted the alignment of seeded bone marrow derived MSCs seeded in a fashion resembling the superficial zone of hyaline cartilage. The cell seeded - triphasic construct led to an improved regeneration of an osteochondral defect in a rabbit model matching the histological scores of autografts [100]. The fully synthetic construct used by Dresing et al.[101] was based on poly(esterurethane) (PUR) in different structures for the regeneration of cartilage, subchondral bone and cortical bone. The cartilage compartment was made by salt leached PUR, the subchondral bone compartment by electrospun PUR, and the bone compartment by a salt leached combination of PUR and nano HA. The bone and cartilage side presented a similar porosity, $85 \%$ and $87 \%$, respectively. The major differences were found in terms of average pore size $121 \mu \mathrm{m}$ and $251 \mu \mathrm{m}$, respectively, and of the addition of the mineral phase resulting in a stiffness of 0.98 $\mathrm{N} / \mathrm{mm}$ for the PUR scaffold alone and of $2.18 \mathrm{~N} / \mathrm{mm}$ for the composite PUR. Despite the elastomeric PUR was easily press fitted in the osteochondral defect, it did not provide any advantage for tissue healing after 12 weeks. Furthermore, this construct could not be really considered as a gradient structure, since the intermediate phase could not be compared with the other two. Additionally, this marked difference between the subchondral bone and the calcified cartilage does not resemble the anatomical transition, in which the two compartment are highly interdigitated [104]. More recently, Jeon et al. [88] developed a system to study osteochondral development ectopically in a nude rat. The desired scaffold to be 
tested was fitted into a cylindrical shaped bovine osteochondral plugs and then implanted subcutaneously in rats. This novel system may allow a higher throughput screening of several osteochondral constructs at the same time than conventional subcutaneous implantation, accounting for the possibility to test up to 8 constructs per animal, thus reducing the number of animals used and associated costs In this study, a multiphasic scaffold was tested, based on bi-layered $2 \%$ alginate containing superficial chondrocyte on the upper part and middle-deep chondrocyte in the lower zone for the cartilage compartment, which was joined with a PCL fused deposition modeling scaffold on top of an electrospun PCL layer, both seeded with osteoblasts, for the bone and interface compartments. Immediately before implantation, the bone compartment was also loaded with rh-BMP7. After 12 weeks, the scaffolds were analyzed. The use of bi-layered cartilage gel, however, didn't recapitulate the compartmental structure of the native cartilage, nor zone specific GAG distribution. Likewise the bone compartment lacked bone ingrowth and mineralization, probably due to insufficient vascularization. Despite the obtained negative results, this study presented an elegant and new method for screening several osteochondral constructs. The division of the scaffold in several zones aimed at resembling the gradient behavior of the osteochondral interface. Yet, one of the main possible limitations in this study was the size of the construct, a $7 \mathrm{~mm}$ long scaffold, which might have encountered some difficulties in integrating with the surrounding tissue, likely due to the lack of associated vasculature.

\subsection{Continuous gradient scaffolds}

In several studies, the Detamore's group proposed and tested an effective method to overcome the limitation encountered in the production of discrete gradient scaffolds [16, 76, 77, 105-107]. The first step consisted in the generation of poly(D,L-lactide-co-glycolide) (PLGA) microspheres in which a desired compound can be added [108]. The loaded microspheres were dispersed in distilled water/PVA solution and placed in two syringes each connected to a pump to control the flow. The suspension was then pumped into a cylindrical mold by varying the flow in order to generate an opposite gradient. The water/PVA was 
pumped out and the particles were soaked in ethanol to facilitate physical attachment among adjacent microspheres. An additional freeze-drying step was then performed. The microspheres were loaded with $\mathrm{CaCO}_{3}$ and $\mathrm{TiO}_{2}$ [107], TGF$\beta 1$ and BMP-2 [106] and chondroitin-sulfate and bioglass [77]. If a single gradient was desired, one pump was loaded with pure PLGA microspheres. The biocompatibility of the system was tested on porcine chondrocytes [108] and human umbilical cord MSCs [107]. When the microspheres were loaded with bioactive signals, cells seeded on gradient scaffolds outperformed the controls after 6 weeks of culture in terms of cell number, protein content and gene expression of osteogenic and chondrogenic markers [106]. After these promising results in vitro, scaffolds based on microspheres loaded with BMP-2 on the bone side and TGF- $\beta 1$ on the cartilage side were implanted in a small mandibular condyle rabbit model [105]. The profile for gradient constructs was linear, such that the transition region from TGF- $\beta 1$ to BMP-2 comprised the second quarter of the scaffold volume, while the top quarter and bottom half contained all TGF- $\beta 1$ or BMP-2-loaded microspheres, respectively. After 6 weeks of implantation, histological and MRI analysis proved the addition of bioactive signals to be less effective than expected. On the cartilage side the mechanical support of the scaffold played a predominant role than the presence of TGF- $\beta 1$ in generating a smoother and thicker cartilage layer when compared with the non-treated defect. BMP-2 led to thicker trabeculae but as well on this side the mechanical support of the scaffold played a greater role, since the subchondral bone layer was more uniform in the gradient and blank scaffolds compared to the non-treated ones. The total bone apposition was not greatly influenced either, probably due to the small size of the defect. An evolution of this system was presented in 2013. The PLGA microspheres were loaded with bioactive signals in combination with components of the ECM. The region of the scaffold designed for osteogenic differentiation presented microspheres loaded with BMP-2 and bioglass, decreasing in number toward the other side of the scaffold, rich in microspheres loaded with TGF- $\beta 3$ and chondroitin sulfate. MSCs displayed a higher deposition of ECM markers when seeded on scaffolds loaded with the raw material in comparison with scaffolds presenting the growth factors alone. Cells seeded in scaffolds containing opposing 
gradients of CS/TGF- $\beta 3$ and BG/BMP-2 produced clear regional variations in the secretion of tissue-specific ECM. Overall, this microsphere-based scaffold proved to be an effective way to incorporate different biological signals of various nature in a scaffold and generate opposite gradients of more than one compound, thus closing the gap to mimic the gradient behavior of the osteochondral tissue.

Most of the relevant studies connected to the use of gradient scaffolds for the treatment of osteochondral defects are involving the use of scaffolds presenting discrete gradients only. Even though the osteochondral interface presents discrete variations when moving from the subchondral bone to the cartilagineous compartment, a continuous gradient is present within hyaline cartilage. A combinatorial approach of the multi-layered technique and the solution proposed by Detamore's group can find, therefore, a possible application in the development of a scaffold resembling even more the characteristics of the osteochondral interface.

a

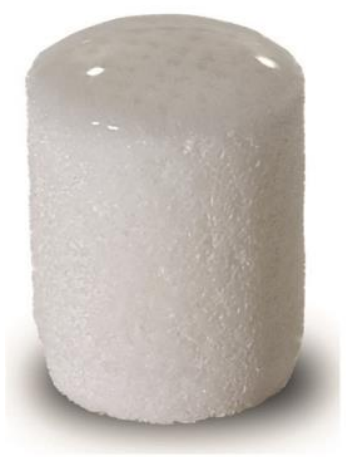

b

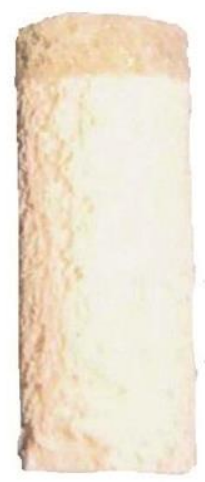

C

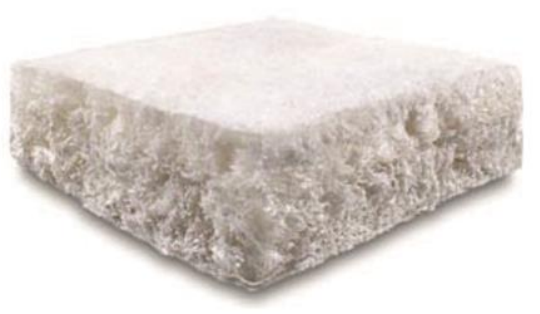

Figure 4. Pictures of the scaffolds which reached the clinical trials, a) Aragonite- based osteochondral scaffold (Agili-CTM, CartiHeal (2009) Ltd, Israel), b) polymeric PLGA-PGA and Calcium sulfate bi-layer scaffold (Trufit CBTM, Smith \& Nephew, USA) and c) HACollagen type I three-layer scaffold (MaioregenTM, Finceramica, Italy). Modified from Elizaveta Kon, 2014.

\subsection{The gradient in the clinics}

Currently, only 3 gradient scaffold designs reached the phase of clinical trials, they are shown in Figure 4. Two of them are bi-phasic scaffolds and the last one is tri- 
phasic scaffold design. TruFit ${ }^{T M}$ (Smith \& Nephew, Andover, MA) is a biphasic scaffold based on PLGA-PGA 75:25 and calcium phosphate, which are glued together with a small amount of solvent after preparation. The cartilage phase was prepared by the addition of $10 \%$ poly(glycolide) reinforcing fibers to a blend of PLGA-PGA 75:25 to improve the compressive modulus [109]. The addition of the bone compartment and the effectiveness of the construct in treating an osteochondral defect was assessed in a goat orthotopic model [110]. The $2.7 \mathrm{~mm}$ high calcium sulfate cylinder was glued with the previously prepared $1.2 \mathrm{~mm}$ high cartilage cylinder, and the glue provided a thin film of $1 \mathrm{~mm}$ in thickness at the articulating surface of the implant. Improved healing of the orthotopic defect was shown after 16 weeks from implantation, with abundant hyaline cartilage formation, integration, and good bony restoration. The addition of chondrocyte to the implant was also tested, but didn't show any additional beneficial effect. Two clinical studies conducted by Bedi et al. in 2010 [111] and Barber et al. in 2011 [112] showed controversial results. The first study described a slow improvement of the injured site. The second clinical study performed a CT scan on 9 patients with intervals between 2 and 63 months, showing no evidence of bone ingrowth, osteoconductivity, or ossification. The decrease in implant density over time reached levels of fibrous scar. Another study from Quarch et al. showed no clinical improvement in the 21 patients treated with this scaffold design [113]. After these results, the scaffold was used only to treat cartilage defects. Positive results were obtained by Carmont et al. [114] on a 18 year old patient with a large chondral defect. The patient returned to sport activities, indicating resolution of pain. Despite these controversial findings, a number of clinical trials were conducted [115-118]. In the study of Bekkers et al. [115], the scaffolds presented newly formed cartilagelike tissue within the implant, which didn't damage the opposing and surrounding tissue after 12 months. Other clinical studies showed in the best case modest improvement [116], but a 70\% failure in active patients in the worst cases [117]. From these studies, a general slow healing process with controversial end results provided by the TruFit ${ }^{\mathrm{TM}}$ plug have been observed, thus justifying for the continuous research for more functional scaffold designs. 
Another biphasic scaffold which reached the clinical practice was developed by Kon et al. (Agili-C ${ }^{\mathrm{TM}}$, CartiHeal (2009) Ltd, Israel). The design was based on calcium carbonate in the form of crystalline aragonite for the bone side and on hyaluronic acid for the cartilage compartment [119]. The first study performed in a goat model aimed at defining the best construct design. The impregnation with hyaluronic acids and the presence of drilled channels were tested. A defect of 6 $\mathrm{mm}$ in diameter and $8 \mathrm{~mm}$ deep was made in the medial femoral condyle and the scaffolds were implanted for 6 months. Scaffolds presenting 2 drilled phases outscored the other groups by displaying a smooth contour and newly formed hyaline cartilage well integrated with the surrounding native cartilage, and with subchondral bone respectively. This construct hits the clinic in a first-in-man experiment. It was used in in a 47 years old non-professional sportsman, resulting in successful treatment of a post traumatic femoral condyle lesion of $2 \mathrm{~cm}^{2}$. After 18 months the man returned to his pre-injury sport activity. MRI analysis at 24 months follow-up showed promising results in terms of restoration of the articular cartilage [120].

Among the multi-layered scaffolds for osteochondral regeneration, a biomimetic construct consisted of different compositions for each zone was also developed by Kon et al. [121]. The cartilage compartment was based on pure collagen type I, a tidemark-like structure was obtained by combining collagen type I and HA in a 60:40 weight ratio, and a mineralized portion corresponding to the subchondral bone presented a 30:70 weight ratio composition of collagen I and HA (FinCeramica, Faenza, Italy). The three components were synthetized separately and combined together on top of a Mylar sheet, freeze dried, and gamma sterilized. The intermediate and lower layer were formed by nucleating bone-like nanostructured non stoichiometric HA into self-assembling collagen fiber, reproducing what happens at the biological level in the neo-ossification process. The resulting construct presented a gradient in mineral content from the bone to the chondral side and a gradient in collagen content in the opposite direction. The first study was performed in a horse animal model, testing the efficacy of bi- and trilayered scaffolds for the regeneration of chondral and osteochondral lesions [122]. The tri-layered scaffolds were produced as mentioned above whereas the bi- 
layered scaffolds were missing the lower part, since they were intended for the regeneration of a chondral damage. After 6 weeks of implantation, both the chondral and osteochondral lesions were filled. The newly formed trabecular bone was visible at the subchondral level and a tide mark-like region was also present. Unfortunately, fibrocartilage and not hyaline cartilage filled the chondral compartment and a first alignment of the collagen fibers was observed. In a subsequent study, the addition of autologous chondrocytes seeded within the trilayered scaffold was studied in a sheep orthotopic model to assess any additional beneficial effect of cells [121]. After a 6 months implantation, the presence of newly formed cartilage in contact with the surrounding tissue was observed in the constructs with and without cells. In the lower compartment, newly-formed subchondral bone was observed. The presence within the subchondral compartment of collagen type II and cells with hypertrophic chondrocyte morphology suggested that the scaffold-mediated regeneration of subchondral bone followed an endochondral ossification process.

The use of the just described biomimetic graded scaffolds were applied in a clinical study for the repair of knee chondral or osteochondral lesion. An early analysis on its stability was carried out in a pilot clinical trial. The first study confirmed the attachment of the graft and only a partial detachment in 2 patients out of 15 . Visual scoring of the repaired tissue revealed a normal repair score in one case and a near-normal repair score in the other cases. Subchondral bone formation without the presence of biomaterial was showed by histological analysis, thus indicating the possible complete resorption of the biomaterial after 6 months. The cartilage tissue appeared not only repaired but engaged in an ongoing maturation process [123]. These encouraging results led to a longer follow-up with randomized studies. A cluster of 27 patients were followed for 2 and 5 years after surgery for knee chondral or osteochondral lesions (size 1.5-6 $\mathrm{cm}^{2}$ ) [124]. Results showed a significant increase of the quality of the regenerated tissue following the International Knee Documentation Committee subjective score. The person's activity level, measured by Tenger scores, depicted an increase from the preoperatory to the 2 and 5 years follow-up. The Tenger scores remained lower than the one before the injury, but didn't reach a statistically significant difference. After 
2 years the cartilage resulted completely filled in $65.2 \%$ of the treated cases, the construct was completely integrated in $69.6 \%$ of the cases, intact repair tissue surface was observed in $56.5 \%$ of the cases, and a homogeneous structure of the repair tissue in $34.8 \%$ of the patients. However, the subchondral lamina and bone resulted intact in only $7 \%$ and $47 \%$ of the cases, respectively [10]. At 5 years follow-up, MRI evaluation revealed a significant improvement in both cartilage and subchondral bone status.

\section{Conclusions}

In the present review, the gradients governing the regeneration of the osteochondral interface were presented alongside with an overview of the solution from the laboratory bench to the bed side. The osteochondral tissue is based on gradients from the early developmental stage until the fully developed body in the adult organism. Nowadays, a lot of effort is made to design scaffolds that lead the healing of the osteochondral interface trying to re-establish the gradient present in the patient. We have presented a few promising methods for the regeneration of the osteochondral tissue. To overcome the abnormalities that still persist after midterm follow-up in clinical trials so far available, a joint work between material scientists, clinicians and developmental biologists should be sought in order to elucidate those mechanisms and pathways involved in the early stage of bone and cartilage development and include those signals into structures able to trigger not only healing of the tissue, but also a proper regeneration. 


\section{References}

1. Dawson, J., et al., Epidemiology of hip and knee pain and its impact on overall health status in older adults. Rheumatology (Oxford), 2004. 43(4): p. 497-504.

2. Dunlop, D.D., et al., Arthritis prevalence and activity limitations in older adults. Arthritis Rheum, 2001. 44(1): p. 212-21.

3. Mannoni, A., et al., Epidemiological profile of symptomatic osteoarthritis in older adults: a population based study in Dicomano, Italy. Annals of the Rheumatic Diseases, 2003. 62(6): p. 576-578.

4. D. Schaefer!, I. Martin, P. Shastri, R.F. Padera, R. Langer, L.E. Freed and G. VunjakNovakovic, In vitro generation of osteochondral composites <1-s2.0-S0142961200001277main.pdf>. Biomaterials, 2000.

5. Martin, I., et al., Osteochondral tissue engineering. J Biomech, 2007. 40(4): p. 750-65.

6. Schek, R.M., et al., Engineered osteochondral grafts using biphasic composite solid free-form fabricated scaffolds. Tissue engineering, 2004. 10(9-10): p. 1376-1385.

7. Shimomura, K., et al., Osteochondral tissue engineering with biphasic scaffold: current strategies and techniques. Tissue Eng Part B Rev, 2014. 20(5): p. 468-76.

8. Grayson, W.L., et al., Spatial regulation of human mesenchymal stem cell differentiation in engineered osteochondral constructs: effects of pre-differentiation, soluble factors and medium perfusion. Osteoarthritis Cartilage, 2010. 18(5): p. 714-23.

9. Levingstone, T.J., et al., A biomimetic multi-layered collagen-based scaffold for osteochondral repair. Acta Biomaterialia, 2014. 10(5): p. 1996-2004.

10. Kon, E., et al., Novel nano-composite multilayered biomaterial for osteochondral regeneration: a pilot clinical trial. Am J Sports Med, 2011. 39(6): p. 1180-90.

11. Aydin, H.M., A Three-Layered Osteochondral Plug: Structural, Mechanical, and in vitro Biocompatibility Analysis. Advanced Engineering Materials, 2011. 13(12): p. B511-B517.

12. Woodfield, T.B., et al., Design of porous scaffolds for cartilage tissue engineering using a three-dimensional fiber-deposition technique. Biomaterials, 2004. 25(18): p. 4149-61.

13. Benders, K.E., et al., Extracellular matrix scaffolds for cartilage and bone regeneration. Trends Biotechnol, 2013. 31(3): p. 169-76.

14. Zhang, Y., et al., Analysis of the Mineral Composition of the Human Calcified Cartilage Zone. International Journal of Medical Sciences, 2012. 9(5): p. 353-360.

15. Oh, S.H., T.H. Kim, and J.H. Lee, Creating growth factor gradients in three dimensional porous matrix by centrifugation and surface immobilization. Biomaterials, 2011. 32(32): p. 8254-60.

16. Milind Singh, C.B., and Michael S. Detamore, Strategies and Applications for Incorporating Physical and Chemical Signal Gradients in Tissue Engineering. TISSUE ENGINEERING: Part B, 2008.

17. Kronenberg, H.M., Developmental regulation of the growth plate. Nature, 2003. 423(6937): p. 332-6.

18. Chimal-Monroy, J. and L. Diaz de Leon, Expression of N-cadherin, $\mathrm{N}$-CAM, fibronectin and tenascin is stimulated by TGF-beta1, beta2, beta3 and beta5 during the formation of precartilage condensations. Int J Dev Biol, 1999. 43(1): p. 59-67.

19. Nakashima, K. and B. de Crombrugghe, Transcriptional mechanisms in osteoblast differentiation and bone formation. Trends Genet, 2003. 19(8): p. 458-66.

20. de Crombrugghe, B., V. Lefebvre, and K. Nakashima, Regulatory mechanisms in the pathways of cartilage and bone formation. Current Opinion in Cell Biology, 2001. 13(6): p. 721-728.

21. Pogue, R. and K. Lyons, BMP signaling in the cartilage growth plate. Curr Top Dev Biol, 2006. 76: p. 1-48.

22. Wang, Y., et al., IGF-1R signaling in chondrocytes modulates growth plate development by interacting with the PTHrP/lhh pathway. J Bone Miner Res, 2011. 26(7): p. 1437-46.

23. Smits, P., et al., Sox5 and Sox6 are needed to develop and maintain source, columnar, and hypertrophic chondrocytes in the cartilage growth plate. J Cell Biol, 2004. 164(5): p. 747-58.

24. Kobayashi, T., et al., PTHrP and Indian hedgehog control differentiation of growth plate chondrocytes at multiple steps. Development, 2002. 129(12): p. 2977-86.

25. Goldring, M.B., K. Tsuchimochi, and K. ljiri, The control of chondrogenesis. Journal of Cellular Biochemistry, 2006. 97(1): p. 33-44. 
26. Pacifici, M., et al., Hypertrophic chondrocytes. The terminal stage of differentiation in the chondrogenic cell lineage? Ann N Y Acad Sci, 1990. 599: p. 45-57.

27. Bianco, P., et al., Bone formation via cartilage models: The "borderline" chondrocyte. Matrix Biology, 1998. 17(3): p. 185-192.

28. Yates, K.E., S. Shortkroff, and R.G. Reish, Wnt influence on chondrocyte differentiation and cartilage function. DNA Cell Biol, 2005. 24(7): p. 446-57.

29. Komiya, Y. and R. Habas, Wnt signal transduction pathways. Organogenesis, 2008. 4(2): p. 68-75.

30. Kühl, M., et al., The Wnt/Ca2+ pathway: a new vertebrate Wnt signaling pathway takes shape. Trends in Genetics, 2000. 16(7): p. 279-283.

31. Leijten, J.C., et al., Gremlin 1, frizzled-related protein, and Dkk-1 are key regulators of human articular cartilage homeostasis. Arthritis Rheum, 2012. 64(10): p. 3302-12.

32. Kawano, Y. and R. Kypta, Secreted antagonists of the Wnt signalling pathway. Journal of Cell Science, 2003. 116(13): p. 2627-2634.

33. Chen, G., C. Deng, and Y.-P. Li, TGF- $\beta$ and BMP Signaling in Osteoblast Differentiation and Bone Formation. International Journal of Biological Sciences, 2012. 8(2): p. 272-288.

34. Heldin, C.H., K. Miyazono, and P. ten Dijke, TGF-beta signalling from cell membrane to nucleus through SMAD proteins. Nature, 1997. 390(6659): p. 465-71.

35. Itoh, F., et al., Promoting bone morphogenetic protein signaling through negative regulation of inhibitory Smads. Vol. 20. 2001. 4132-4142.

36. Lin, A.H., et al., Global analysis of Smad2/3-dependent TGF-beta signaling in living mice reveals prominent tissue-specific responses to injury. J Immunol, 2005. 175(1): p. 547-54.

37. Furumatsu, T., et al., Smad3 Induces Chondrogenesis through the Activation of SOX9 via CREB-binding Protein/p300 Recruitment. Journal of Biological Chemistry, 2005. 280(9): p. 8343-8350.

38. Mundy, G.R., Nutritional modulators of bone remodeling during aging. Am J Clin Nutr, 2006. 83(2): p. 427S-430S.

39. Kwan Tat, S., et al., Treatment with ephrin B2 positively impacts the abnormal metabolism of human osteoarthritic chondrocytes. Arthritis Res Ther, 2009. 11(4): p. R119.

40. Hunziker, E.B., T.M. Quinn, and H.J. Hauselmann, Quantitative structural organization of normal adult human articular cartilage. Osteoarthritis Cartilage, 2002. 10(7): p. 564-72.

41. Bobinac, D., et al., Changes in articular cartilage and subchondral bone histomorphometry in osteoarthritic knee joints in humans. Bone, 2003. 32(3): p. 284-290.

42. Sharma, A., et al., Interplay between Cartilage and Subchondral Bone Contributing to Pathogenesis of Osteoarthritis. International Journal of Molecular Sciences, 2013. 14(10): p. 19805-19830.

43. Heinegard, D. and A. Oldberg, Structure and biology of cartilage and bone matrix noncollagenous macromolecules. FASEB J, 1989. 3(9): p. 2042-51.

44. Harada, S. and G.A. Rodan, Control of osteoblast function and regulation of bone mass. Nature, 2003. 423(6937): p. 349-55.

45. Knothe Tate, M.L., et al., The osteocyte. The International Journal of Biochemistry \& Cell Biology, 2004. 36(1): p. 1-8.

46. Oegema, T.R., Jr., et al., The interaction of the zone of calcified cartilage and subchondral bone in osteoarthritis. Microsc Res Tech, 1997. 37(4): p. 324-32.

47. Bhosale, A.M. and J.B. Richardson, Articular cartilage: structure, injuries and review of management. Br Med Bull, 2008. 87: p. 77-95.

48. Farnum, C.E., et al., Volume increase in growth plate chondrocytes during hypertrophy: the contribution of organic osmolytes. Bone, 2002. 30(4): p. 574-581.

49. Mason, R.M., Recent advances in the biochemistry of hyaluronic acid in cartilage. Prog Clin Biol Res, 1981. 54: p. 87-112.

50. Poole, C.A., M.H. Flint, and B.W. Beaumont, Morphological and functional interrelationships of articular cartilage matrices. Journal of Anatomy, 1984. 138(Pt 1): p. 113-138.

51. Miosge, N., et al., Light and electron microscopical immunohistochemical localization of the small proteoglycan core proteins decorin and biglycan in human knee joint cartilage. Histochem J, 1994. 26(12): p. 939-45.

52. Glant, T.T., et al., Appearance and persistence of fibronectin in cartilage. Specific interaction of fibronectin with collagen type II. Histochemistry, 1985. 82(2): p. 149-58.

53. Poole, C.A., Review. Articular cartilage chondrons: form, function and failure. Journal of Anatomy, 1997. 191(Pt 1): p. 1-13. 
54. Durr, J., et al., Identification and immunolocalization of laminin in cartilage. Exp Cell Res, 1996. 222(1): p. 225-33.

55. Blitterswijk, C.V., Tissue Engineering. book.

56. Musumeci, G., et al., Lubricin expression in human osteoarthritic knee meniscus and synovial fluid: a morphological, immunohistochemical and biochemical study. Acta Histochem, 2014. 116(5): p. 965-72.

57. Buckwalter, J.A., V.C. Mow, and A. Ratcliffe, Restoration of Injured or Degenerated Articular Cartilage. J Am Acad Orthop Surg, 1994. 2(4): p. 192-201.

58. Nilsson, O., et al., Gradients in bone morphogenetic protein-related gene expression across the growth plate. J Endocrinol, 2007. 193(1): p. 75-84.

59. Gamer, L.W., et al., Expression and Function of BMP3 During Chick Limb Development. Developmental dynamics : an official publication of the American Association of Anatomists, 2008. 237(6): p. 1691-1698.

60. MANSOUR, J.M., Biomechanics of Cartilage.

61. Hunziker, E.B., Articular cartilage repair: basic science and clinical progress. A review of the current status and prospects. Osteoarthritis and Cartilage, 2002. 10(6): p. 432-463.

62. Hayes, A.J., et al., The development of articular cartilage: evidence for an appositional growth mechanism. Anat Embryol (Berl), 2001. 203(6): p. 469-79.

63. Mente, P.L. and J.L. Lewis, Elastic modulus of calcified cartilage is an order of magnitude less than that of subchondral bone. J Orthop Res, 1994. 12(5): p. 637-47.

64. Rolauffs, B., et al., Vulnerability of the superficial zone of immature articular cartilage to compressive injury. Arthritis and rheumatism, 2010. 62(10): p. 3016-3027.

65. Treppo, S., et al., Comparison of biomechanical and biochemical properties of cartilage from human knee and ankle pairs. Journal of Orthopaedic Research, 2000. 18(5): p. 739-748.

66. Moroni, L., J.R. de Wijn, and C.A. van Blitterswijk, 3D fiber-deposited scaffolds for tissue engineering: Influence of pores geometry and architecture on dynamic mechanical properties. Biomaterials, 2006. 27(7): p. 974-985.

67. O'Hara, B.P., J.P. Urban, and A. Maroudas, Influence of cyclic loading on the nutrition of articular cartilage. Annals of the Rheumatic Diseases, 1990. 49(7): p. 536-539.

68. Zhou, S., Z. Cui, and J.P. Urban, Nutrient gradients in engineered cartilage: metabolic kinetics measurement and mass transfer modeling. Biotechnol Bioeng, 2008. 101(2): p. 408-21.

69. Prydz, K. and K.T. Dalen, Synthesis and sorting of proteoglycans. Journal of cell science, 2000. 113(2): p. 193-205.

70. Lund-Olesen, K., Oxygen tension in synovial fluids. Arthritis \& Rheumatism, 1970. 13(6): p. 769-776.

71. Falchuk, K.H., E.J. Goetzl, and J.P. Kulka, Respiratory gases of synovial fluids: An approach to synovial tissue circulatory-metabolic imbalance in rheumatoid arthritis. The American Journal of Medicine, 1970. 49(2): p. 223-231.

72. Fermor, B., et al., Oxygen, nitric oxide and articular cartilage. Eur Cell Mater, 2007. 13: p. 5665; discussion 65.

73. Silver, I.A., Measurement of $\mathrm{pH}$ and ionic composition of pericellular sites. Philos Trans $\mathrm{R}$ Soc Lond B Biol Sci, 1975. 271(912): p. 261-72.

74. Sobral, J.M., et al., Three-dimensional plotted scaffolds with controlled pore size gradients: Effect of scaffold geometry on mechanical performance and cell seeding efficiency. Acta Biomater, 2011. 7(3): p. 1009-18.

75. Woodfield, T.B., et al., Polymer scaffolds fabricated with pore-size gradients as a model for studying the zonal organization within tissue-engineered cartilage constructs. Tissue Eng, 2005. 11(9-10): p. 1297-311.

76. Mohan, N., et al., Continuous gradients of material composition and growth factors for effective regeneration of the osteochondral interface. Tissue Eng Part A, 2011. 17(21-22): p. 2845-55.

77. Neethu Mohan, V.G., BanuPriya Sridharan, Amanda Sutherland, Michael S. Detamore, The Potential of Encapsulating "Raw Materials" in 3D Osteochondral Gradient Scaffolds. Biotechnol. Bioeng. , 2014. 111: p. 829-841.

78. Milind Singh, N.D., Jean R. Salash, Jordan M. Christian, David S. Moore, Cory Berkland, Michael S. Detamore, Three-dimensional macroscopic scaffolds with a gradient in stiffness for functional regeneration of interfacial tissues. WILEY PERIODICALS, 2010.

79. Elizaveta Kon*, G.F., Francesco Perdisa, Giulia Venieri and Maurilio Marcacci, Clinical results of multilayered biomaterials for osteochondral regeneration. 2014. 
80. Zhao, W., et al., Degradable natural polymer hydrogels for articular cartilage tissue engineering. Journal of Chemical Technology \& Biotechnology, 2013. 88(3): p. 327-339.

81. Solchaga, L.A., et al., Repair of osteochondral defects with hyaluronan- and polyester-based scaffolds. Osteoarthritis Cartilage, 2005. 13(4): p. 297-309.

82. Ding, C., et al., Regeneration of a goat femoral head using a tissue-specific, biphasic scaffold fabricated with CAD/CAM technology. Biomaterials, 2013. 34(28): p. 6706-16.

83. TONG CAO, K.-H.H.a.S.-H.T., Scaffold Design and in Vitro Study of Osteochondral Coculture in a Three-Dimensional Porous Polycaprolactone Scaffold Fabricated by Fused Deposition Modeling. TISSUE ENGINEERING, 2003. 9.

84. Kandel, R.A., et al., Repair of osteochondral defects with biphasic cartilage-calcium polyphosphate constructs in a Sheep model. Biomaterials, 2006. 27(22): p. 4120-4131.

85. Duan, P., et al., The effects of pore size in bilayered poly(lactide-co-glycolide) scaffolds on restoring osteochondral defects in rabbits. J Biomed Mater Res A, 2013.

86. Chen, J., et al., Simultaneous regeneration of articular cartilage and subchondral bone in vivo using MSCs induced by a spatially controlled gene delivery system in bilayered integrated scaffolds. Biomaterials, 2011. 32(21): p. 4793-4805.

87. Re'em, T., et al., Simultaneous regeneration of articular cartilage and subchondral bone induced by spatially presented TGF-beta and BMP-4 in a bilayer affinity binding system. Acta Biomater, 2012. 8(9): p. 3283-93.

88. Jeon, J.E., et al., Multiphasic construct studied in an ectopic osteochondral defect model. J R Soc Interface, 2014. 11(95): p. 20140184.

89. Heymer, A., et al., Multiphasic collagen fibre-PLA composites seeded with human mesenchymal stem cells for osteochondral defect repair: an in vitro study. Journal of Tissue Engineering and Regenerative Medicine, 2009. 3(5): p. 389-397.

90. Sundelacruz, S. and D.L. Kaplan, Stem cell- and scaffold-based tissue engineering approaches to osteochondral regenerative medicine. Seminars in Cell \& Developmental Biology, 2009. 20(6): p. 646-655.

91. Anderson, J.A., et al., Stem Cell Therapies for Knee Cartilage Repair: The Current Status of Preclinical and Clinical Studies. The American Journal of Sports Medicine, 2014. 42(9): p. 2253-2261.

92. Panseri, S., et al., Osteochondral tissue engineering approaches for articular cartilage and subchondral bone regeneration. Knee Surgery, Sports Traumatology, Arthroscopy, 2012. 20(6): p. 1182-1191.

93. Budiraharjo, R., K.G. Neoh, and E.T. Kang, Enhancing bioactivity of chitosan film for osteogenesis and wound healing by covalent immobilization of BMP-2 or FGF-2. J Biomater Sci Polym Ed, 2013. 24(6): p. 645-62.

94. Even-Ram, S., V. Artym, and K.M. Yamada, Matrix control of stem cell fate. Cell, 2006. 126(4): p. 645-7.

95. Justin R. Tse, A.J.E., Stiffness Gradients Mimicking In Vivo Tissue Variation Regulate Mesenchymal Stem Cell Fate. Plos One, 2011.

96. Sherwood, J.K., et al., A three-dimensional osteochondral composite scaffold for articular cartilage repair. Biomaterials, 2002. 23(24): p. 4739-4751.

97. Jiang, J., et al., Bioactive Stratified Polymer Ceramic-Hydrogel Scaffold for Integrative Osteochondral Repair. Annals of Biomedical Engineering, 2010. 38(6): p. 2183-2196.

98. Heymer, A., et al., Multiphasic collagen fibre-PLA composites seeded with human mesenchymal stem cells for osteochondral defect repair: an in vitro study. J Tissue Eng Regen Med, 2009. 3(5): p. 389-97.

99. Marquass, B., et al., A novel MSC-seeded triphasic construct for the repair of osteochondral defects. Journal of Orthopaedic Research, 2010. 28(12): p. 1586-1599.

100. Liu, X., et al., Evaluation of oriented electrospun fibers for periosteal flap regeneration in biomimetic triphasic osteochondral implant. J Biomed Mater Res B Appl Biomater, 2014. 102(7): p. 1407-14.

101. Dresing, I., et al., Evaluation of a press-fit osteochondral poly(ester-urethane) scaffold in a rabbit defect model. Journal of Materials Science: Materials in Medicine, 2014. 25(7): p. 16911700.

102. Re'em, T., et al., Simultaneous regeneration of articular cartilage and subchondral bone induced by spatially presented TGF-beta and BMP-4 in a bilayer affinity binding system. Acta Biomaterialia, 2012. 8(9): p. 3283-3293. 
103. Harley, B.A., et al., Design of a multiphase osteochondral scaffold III: Fabrication of layered scaffolds with continuous interfaces. Journal of Biomedical Materials Research Part A, 2010. 92A(3): p. 1078-1093.

104. Ferguson, V.L., A.J. Bushby, and A. Boyde, Nanomechanical properties and mineral concentration in articular calcified cartilage and subchondral bone. Journal of Anatomy, 2003. 203(2): p. 191-202.

105. Dormer, N.H., et al., Osteochondral Interface Regeneration of Rabbit Mandibular Condyle With Bioactive Signal Gradients. Journal of Oral and Maxillofacial Surgery, 2011. 69(6): p. e50-e57.

106. Dormer, N.H., et al., Osteochondral Interface Tissue Engineering Using Macroscopic Gradients of Bioactive Signals. Annals of biomedical engineering, 2010. 38(6): p. 2167-2182.

107. Singh, M., et al., Three-dimensional macroscopic scaffolds with a gradient in stiffness for functional regeneration of interfacial tissues. J Biomed Mater Res A, 2010. 94(3): p. 870-6.

108. Singh, M., et al., Microsphere-based seamless scaffolds containing macroscopic gradients of encapsulated factors for tissue engineering. Tissue Eng Part C Methods, 2008. 14(4): p. 299309.

109. Slivka, M.A., et al., Porous, resorbable, fiber-reinforced scaffolds tailored for articular cartilage repair. Tissue Eng, 2001. 7(6): p. 767-80.

110. Niederauer, G.G., et al., Evaluation of multiphase implants for repair of focal osteochondral defects in goats. Biomaterials, 2000. 21(24): p. 2561-74.

111. Bedi, A., et al., The Maturation of Synthetic Scaffolds for Osteochondral Donor Sites of the Knee: An MRI and T2-Mapping Analysis. Cartilage, 2010. 1(1): p. 20-28.

112. Barber, F.A. and W.D. Dockery, A Computed Tomography Scan Assessment of Synthetic Multiphase Polymer Scaffolds Used for Osteochondral Defect Repair. Arthroscopy: The Journal of Arthroscopic \& Related Surgery, 2011. 27(1): p. 60-64.

113. Quarch, V.M., et al., Fate of large donor site defects in osteochondral transfer procedures in the knee joint with and without TruFit plugs. Arch Orthop Trauma Surg, 2014. 134(5): p. 65766.

114. Carmont, M.R., et al., Delayed Incorporation of a TruFit Plug: Perseverance Is Recommended. Arthroscopy: The Journal of Arthroscopic \& Related Surgery, 2009. 25(7): p. 810-814.

115. Bekkers, J.E., et al., Articular cartilage evaluation after TruFit plug implantation analyzed by delayed gadolinium-enhanced MRI of cartilage (dGEMRIC). Am J Sports Med, 2013. 41(6): $p$. 1290-5.

116. Dhollander, A.A., et al., A pilot study of the use of an osteochondral scaffold plug for cartilage repair in the knee and how to deal with early clinical failures. Arthroscopy, 2012. 28(2): p. 225-33.

117. Joshi, N., et al., Synthetic resorbable scaffolds for the treatment of isolated patellofemoral cartilage defects in young patients: magnetic resonance imaging and clinical evaluation. Am J Sports Med, 2012. 40(6): p. 1289-95.

118. Hindle, P., et al., Autologous osteochondral mosaicplasty or TruFit plugs for cartilage repair. Knee Surg Sports Traumatol Arthrosc, 2014. 22(6): p. 1235-40.

119. Kon, E., et al., Osteochondral regeneration using a novel aragonite-hyaluronate bi-phasic scaffold in a goat model. Knee Surgery, Sports Traumatology, Arthroscopy, 2014. 22(6): p. 1452-1464.

120. Kon, E., et al., Chronic posttraumatic cartilage lesion of the knee treated with an acellular osteochondral-regenerating implant: case history with rehabilitation guidelines. J Sport Rehabil, 2014. 23(3): p. 270-5.

121. Kon, E., et al., Orderly osteochondral regeneration in a sheep model using a novel nanocomposite multilayered biomaterial. Journal of Orthopaedic Research, 2009. 28(1): p. 116124.

122. Kon, E., et al., Novel nanostructured scaffold for osteochondral regeneration: pilot study in horses. J Tissue Eng Regen Med, 2010. 4(4): p. 300-8.

123. Kon, E., et al., $A$ novel nano-composite multi-layered biomaterial for treatment of osteochondral lesions: technique note and an early stability pilot clinical trial. Injury, 2010. 41(7): p. 693-701.

124. Kon, E., et al., Clinical results and MRI evolution of a nano-composite multilayered biomaterial for osteochondral regeneration at 5 years. Am J Sports Med, 2014. 42(1): p. 158-65. 


\section{CHAPTER 2}

\section{Gradients in pore size enhance the osteogenic differentiation of human mesenchymal stromal cells in three-dimensional scaffolds}

Andrea Di Luca, Barbara Ostrowska, Ivan Lorenzo-Moldero, Antonio Lepedda, Wojcech Swieszkowski, Clemens Van Blitterswijk, Lorenzo Moroni 


\begin{abstract}
Small fractures in bone tissue can heal by themselves, but in case of larger defects current therapies are not completely successful due to several drawbacks. A possible strategy relies on the combination of additive manufactured polymeric scaffolds and human mesenchymal stromal cells (hMSCs). The architecture of bone tissue is characterized by a structural gradient. Long bones display a structural gradient in the radial direction, while flat bones in the axial direction. Such gradient presents a variation in bone density from the cancellous bone to the cortical bone. Therefore, scaffolds presenting a gradient in porosity could be ideal candidates to improve bone tissue regeneration. In this study, we present a construct with a discrete gradient in pore size and characterize its ability to further support the osteogenic differentiation of hMSCs. Furthermore, we studied the behaviour of hMSCs within the different compartments of the gradient scaffolds, showing a correlation between osteogenic differentiation and ECM mineralization, and pore dimensions. Alkaline phosphatase activity and calcium content increased with increasing pore dimensions. Our results indicate that designing structural porosity gradients may be an appealing strategy to support gradual osteogenic differentiation of adult stem cells.
\end{abstract}




\section{Introduction}

Regenerative medicine is a multidisciplinary field aiming to regenerate tissues by combining biological factors and engineering fundamentals ${ }^{1}$. Recently the use of stem cells in regenerative medicine has gained momentum thanks to their capacity to differentiate into multiple lineages ${ }^{2,3}$. Human mesenchymal stem/stromal cells (hMSCs) can undergo chondrogenic, osteogenic and adipogenic differentiation ${ }^{3}$, among others, and are not associated to the ethical concerns of other stem cells like embronic ones. hMSC differentiation has been reported to depend on environmental cues such as oxygen and nutrient availability ${ }^{4,5}$, pore size ${ }^{6,7}$, material stiffness ${ }^{8}$, surface topography ${ }^{6,9}$ and more conventionally the adminisration of soluble factors ${ }^{10-12}$. All these parameters have been applied in the design and fabrication of scaffolds aiming at mimicking the natural three-dimensional (3D) enviroment where hMSCs reside. Different processing techniques have been developed to build scaffolds for tissue engineered constructs ${ }^{13}$, among which solvent casting, salt or particulate leaching and gas foaming ${ }^{14}$. Although all these techniques are easy to implement, the resulting scaffolds present several drawbacks, such as the lack of completely interconnected pores, a limited control of the pore size and geometry, and the formation of tortuous pore networks associated to limited nutrient diffusion ${ }^{15,16}$. Conversely, additive manufacturing (AM) emerged in the past decade as an appealing tool to fabricate scaffolds with a controlled and completely interconnected pore network. This can be achieved thanks to the possibility to fine tune processing parameters such as fiber diameter, fiber spacing, layer thickness, and layer angle deposition. Additionally, the computer aided design/computer aided manufacturing (CAD/CAM) process governing AM technologies allows tailoring pore geometry and size in a layer-by-layer manner 17,18. These parameters can be modulated in order to obtain a constant variation of the pore features within the same construct, thus forming structural gradients.

Gradients are present in the body leading a number of events and processes in the embryonic stage as well as in adult life. Structural gradients can be found in 
the body mainly at the interface between tissues. For example, processes such as osteochondral, tendon and ligament tissue development, as well as tumor formation, are governed by morphogens and oxygen gradients ${ }^{19-21}$. In the specific case of bone tissues, a structural gradient can be identified in a radial direction in long bones and in an axial direction in flat bones, presenting a variation in bone density from the cancellous bone to the coritical bone ${ }^{22}$. Clinically, current therapies for bone replacement, such as autograft and allograft, are not yet completely successful, due to several drawbacks such as the donor-site morbidity, the limited tissue availability and surgery complications, highlighting that this procedures are not always a possible option ${ }^{23,24}$.

The concept of gradient has been applied in different studies in two dimensional (2D) systems to control or analyze cell differentiation ${ }^{10}$ and migration ${ }^{25,26}$. 3D scaffolds presenting a gradient structure could provide cues similar to the native enviroment and may guide stem cells to differentiate toward the lineage of the targeted tissue to be regenerated. In literature, several studies involving gradient scaffolds have been presened. In order to direct the differentiation of hMSCs in certain areas of the construct, gradients of growth factors ${ }^{27}$ and material stiffness ${ }^{28}$ were generated. To the best of our knowledge, no studies have linked the stem cell osteogenic differentiation with structural gradients in porosity and pore size. Besides improving cell seeding efficiency due to the higher number of fiber connections ${ }^{29}$, structural gradients can result in locally different concentrations of available nutrients. Therefore, we hypothesized that the creation of a gradient in scaffold porosity and pore size could influence hMSC differentiation by impacting cell density and nutrient availability. Here, we fabricated $3 \mathrm{D}$ plotted scaffolds presenting an axial gradient in pore size and total porosity and assessed their effect on hMSC osteogenic differentiation. 


\section{Results}

\subsection{Scaffold and gradient characterization}

Four zones in the gradient scaffolds can be distinguished (Figure 1c and 1f), where the fiber spacing changed from bottom to top from $500 \mu \mathrm{m}$, to $700 \mu \mathrm{m}, 900 \mu \mathrm{m}$, $1100 \mu \mathrm{m}$. Control scaffolds were printed by keeping the fiber spacing constant at $500 \mu \mathrm{m}$ and $1100 \mu \mathrm{m}$ (Figure 1b, 1e and 1c, 1f). By increasing the fiber spacing, the volume of the pore in the different zones increased by 10 times from the smallest to the largest pore size. (Table S1). As expected, the overall porosity of the gradient scaffolds was in between the porosity of the controls (Table S2). The porosity of the different gradient areas increased from $58 \% \pm 0.07 \%$ close to the porosity of the NG $500(47.24 \% \pm 6.9 \%)$, to $81 \% \pm 0.04 \%$ that matched with porosity values of NG $1100(80.63 \% \pm 2.3 \%)$.
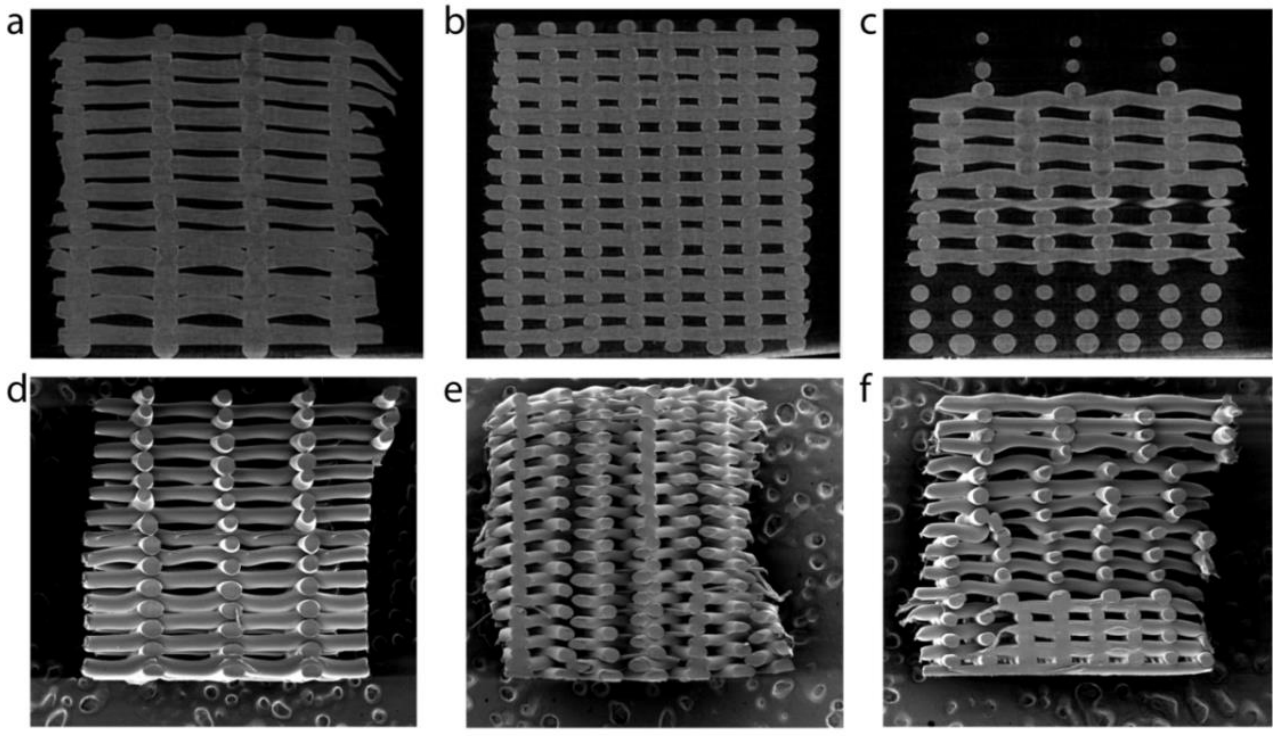

Figure 1. Figure 1. $\mu C T$ and SEM micrographs displaying NG1100 (a,d), NG500 (b,e) and G scaffolds $(\mathrm{c}, \mathrm{f})$. Scale bar $2 \mathrm{~mm}$. 


\section{2. hMSC growth and Improved osteogenic differentiation in the gradient scaffolds}

The amount of cells adhered on 300PEOT55PBT45 and PCL was around 325000 and 250000 cells per scaffold, respectively, which corresponded to a $65 \%$ cell seeding efficiency for 300PEOT55PBT45 and 50\% cell seeding efficiency for PCL (Figures S1 and S2). Though not statistically different, 300PEOT55PBT45 seemed to perform better in terms of cell attachment. After 8 days from cell seeding, cell number on 300PEOT55PBT45 scaffolds remained constant around 250000 cells, without major differences among the conditions or type of construct. The overall cell number did not vary significantly at day 35 , independently from the culture conditions or the type of construct (Figure 2a), thus inferring that hMSCs did not proliferate significantly in these $3 \mathrm{D}$ scaffolds during the culture period.

hMSCs showed a basal ALP level (Figure 2b). After 8 days of culture ( 7 days in proliferation medium and 1 day in basic or mineralization media), as expected, no main differences could be detected between the $G$ and NG scaffolds cultured in basic and mineralization media. The basal ALP levels decreased in time in basic media in NG and G scaffolds. After 28 days in mineralization media the ALP levels markedly increased in all the samples. hMSCs cultured in G scaffolds showed significantly increased ALP activity levels with respect to the cells cultured in the NG scaffolds (Figure 2b). As shown in Figure 2c, after 28 days under mineralization conditions the expression of late osteogenic markers differentiation such as osteocalcin and osteopontin was not increased. Early markers such as bone sialoprotein (BSP) and ALP were upregulated. Bone sialoprotein showed a 5fold increase with respect to the cells cultured in basic medium within the NG 500 construct. No major differences were noticed among the gradient scaffolds and the controls. The expression of ALP was increased by 12 times. Yet, no significant differences could be seen. 


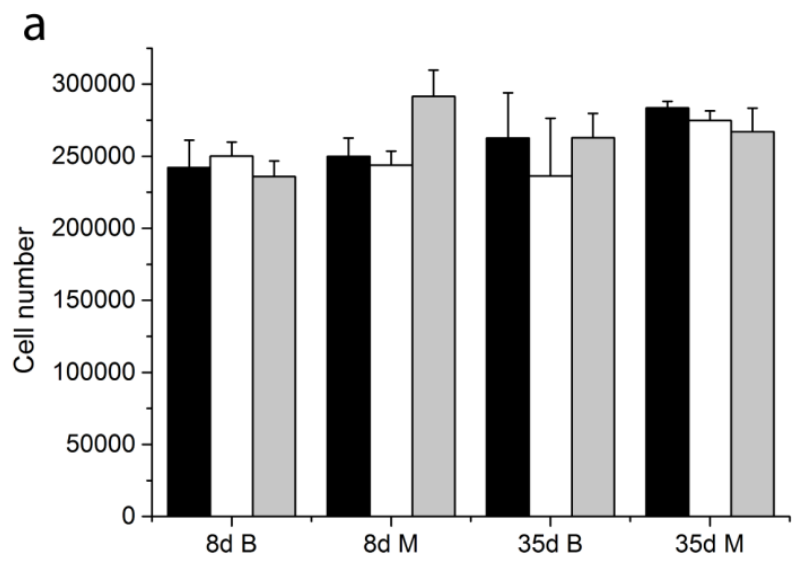

b
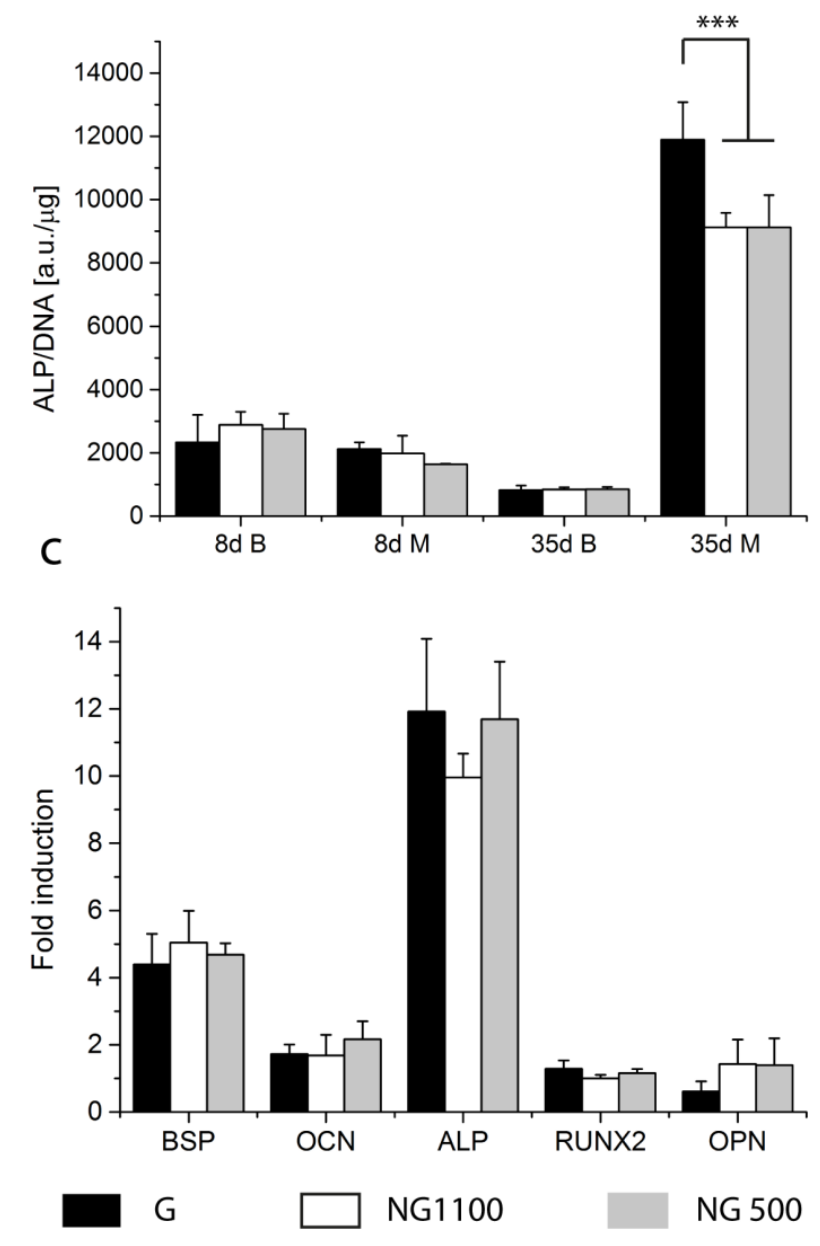
Figure 2. Cell number and ALP activity of D1 on 300PEOT55PBT45 normalized by $\mu \mathrm{g}$ of DNA after 8 and 35 days in culture $(a, b)$ and fold induction of osteogenic markers after 35 days (c). After 1 day in differentiation media (8 days in culture) no differences in ALP activity or cell number were visible, whereas 4 weeks of differentiation enhanced ALP activity in gradient scaffolds with respect to the controls. The cell number remained similar in all conditions. BSP, OCN and ALP genes were upregulated, no major differences were shown among the gradient and non-gradient scaffolds. ${ }^{* * *}$ shows significant difference, $p<0.001$, $n=3)$.

In order to confirm the results obtained in terms of osteogenic differentiation, another hMSC donor was tested in G, NG 500 and NG 1100 scaffolds. As the main differences were seen after 28 days in differentiation condition only this time point was tested. Cell numbers as well as the ALP activity in all the constructs were lower compared to the first donor. Yet, ALP activity was significantly higher in $G$ scaffolds than in the controls (Figure $3 b$ ), thus confirming what observed for the first donor. If the results of the 2 donors are normalized by the NG 1100 or NG 500 values, the second donor displayed a higher induction factor with a 2.5 fold increase in G scaffolds compared to NG scaffolds. The first donor showed an increased ALP activity of $25 \%$ in G scaffolds compared to NG ones (Figure S3). Cell number under basic conditions was significantly lower in NG1100, whereas under mineralization conditions the NG scaffolds showed a higher cell number compared to the $\mathrm{G}$ scaffolds (Figure 3a). In order to understand whether the scaffold material chemistry could influence the differentiation, the same analysis was conducted on scaffolds made of PCL. Despite lack of statistical significance, both cell number and ALP activity seemed to be higher in the control NG 500 compared to NG 1100. The gradient showed values that resembled the ones of the NG 500 control (Figure 3c,d). In addition, osteocalcin production also increased in mineralization medium in G and NG 1100 scaffolds compared to NG 500 scaffolds (Figure S4). 

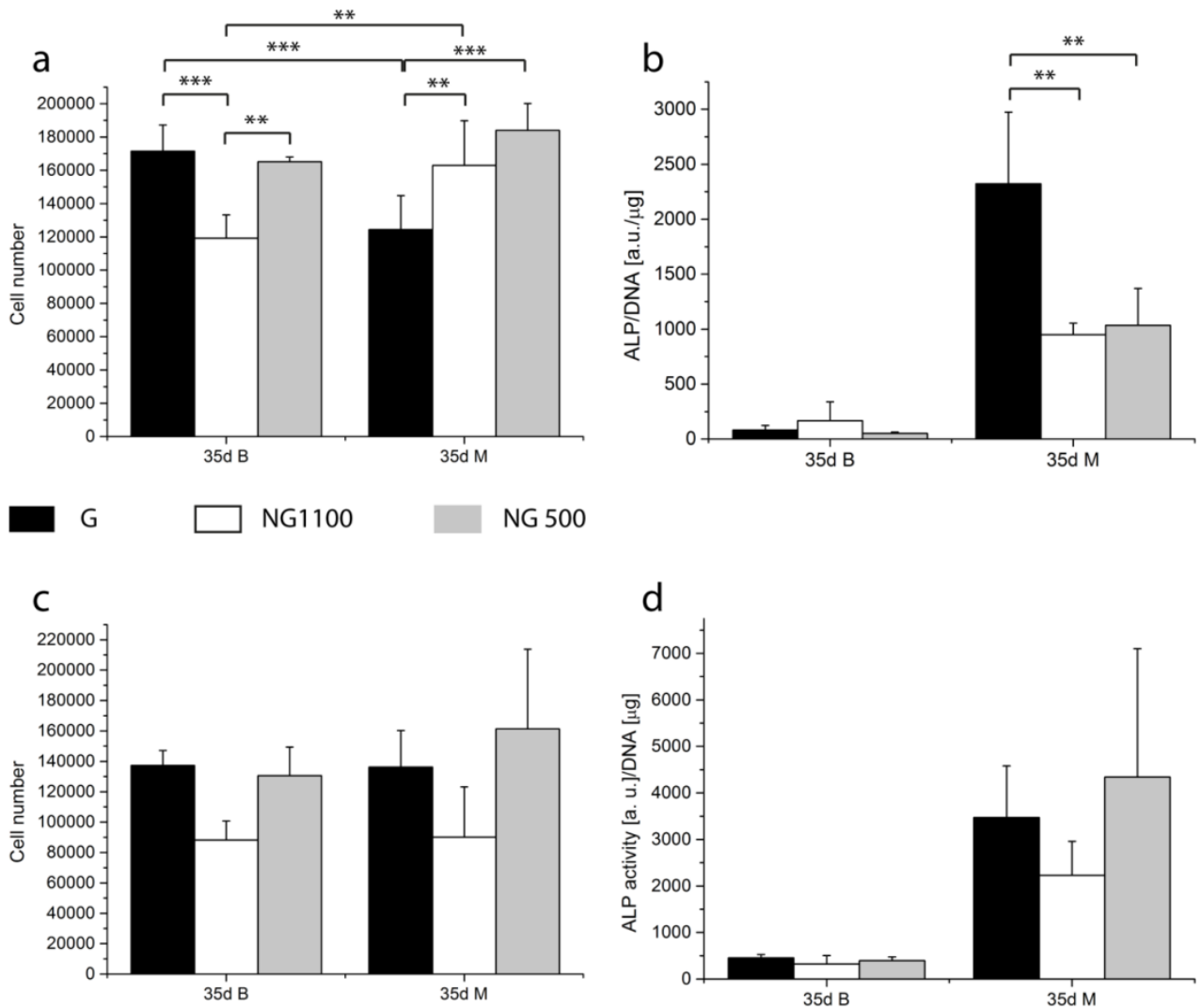

Figure 3. Cell number and ALP activity of donor 2 on 300PEOT55PBT45 scaffolds (a,b) and donor 1 on PCL scaffolds (c,d). Under basic conditions NG1100 scaffolds displayed a significantly lower cell number after 35 days of culture; under mineralization conditions the gradient showed a lower cell number. The ALP activity confirmed the results showed by donor 1 on 300PEOT55PBT45 scaffolds, the gradient significantly improved the ALP activity with respect to the non gradient scaffolds. When cultured on PCL scaffolds hMSCs didn't display any significant differences in terms of cell number and ALP activity in basic as well as in mineralization medium. ${ }^{* *}$ shows statistical significance $p<0.01$ and ${ }^{* * *} p<0.001$.

\subsection{Pore size driven differentiation of hMSCs}

As the overall ALP activity level increased in the $G$ scaffolds compared to the controls, a partition analysis on the different areas of the gradient scaffolds was performed to better understand the differences among the porosity zones of the $G$ scaffold. After 8 days cells were localized mainly in the zone with the lowest porosity independently from the media used (Figure 4a). The ALP activity of cells localized in the small pore zone was higher with respect to the other areas after 1 
day in basic and mineralization media (Figure 4b). This trend was inverted after culturing in differentiation media. ALP activity levels of the cells cultured in mineralization medium increased over time in all the partitioned areas (Figure 4b). The ALP activity increased with increasing the pore size.

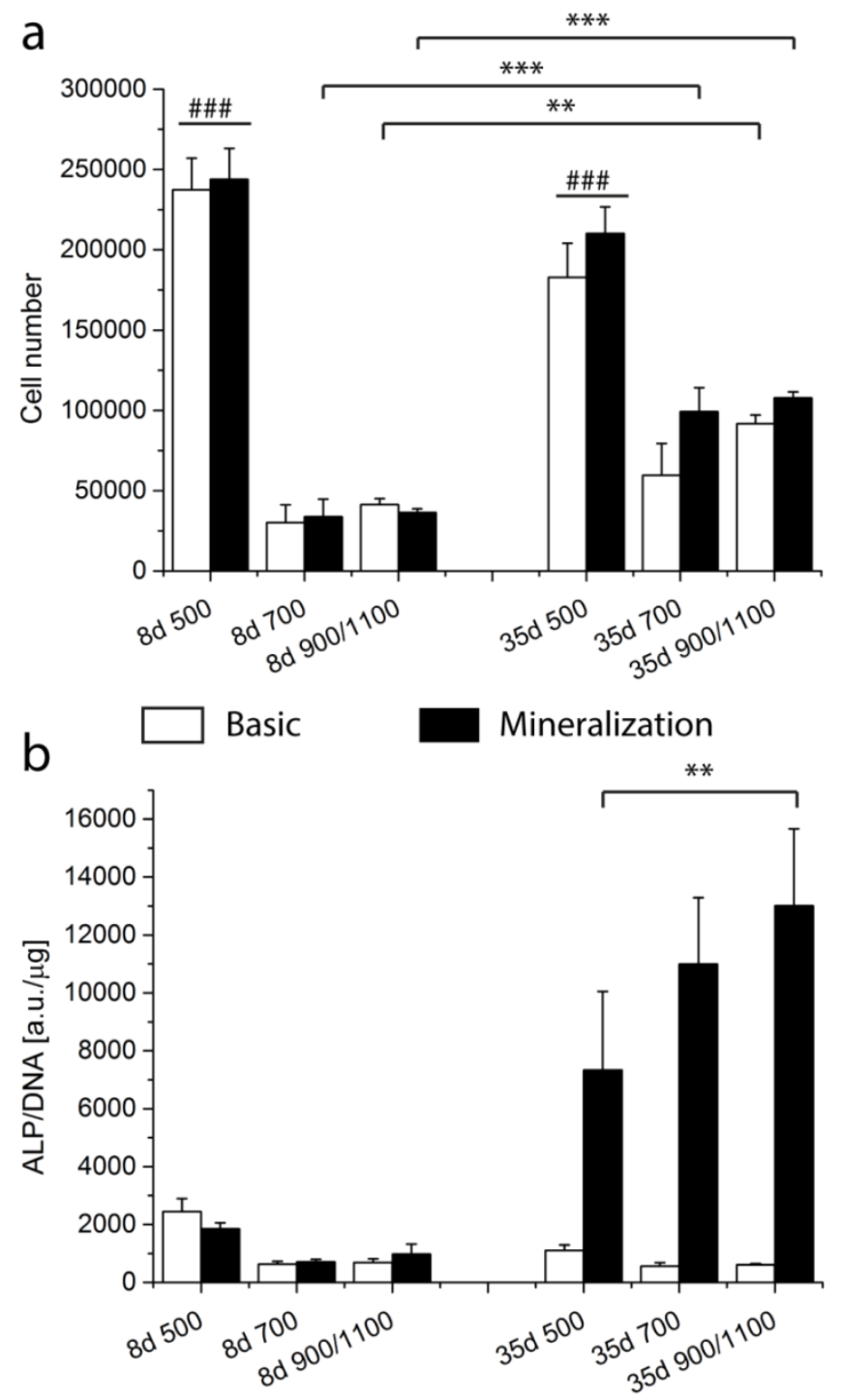

Figure 4. Cell number (a) and ALP activity (b) of D1 on 300PEOT55PBT45 are shown per gradient zone at 8 and 35 days. The cell number was significantly higher in the smallest pore size compared to the other areas no matter neither the time point nor the culture conditions. At 35 days of culture the cell number in the 700 and 900/1100 zone significantly 
increased with respect to the previous time point. The ALP activity at 35 days under mineralization conditions showed an increase trend opposite to the pore size. Cells residing in the 900/1100 zones displayed a significantly higher ALP activity with respect to the ones located in the smallest pore zone. \#\#\# indicates statistical significance within the same time point in graph a, $\mathrm{p}<0.001 ;{ }^{* *},{ }^{* *}$ depict statistical significance within the same conditions $p<0.01$ and $p<0.001$ respectively.

Also for the partition analysis, the second donor and scaffolds in PCL were tested. After 35 days of culture in both conditions cells were located in higher number in the small pore zones for both donors and materials (Figure $5 \mathrm{a}, \mathrm{c}$ ). Cells showed an increase in ALP activity with the increase in pore size for donor 1 in PCL (Figure 5b). D2 in 300PEOT55PBT45 confirmed the lowest ALP levels where pores were small. No major differences could be seen between the 700 and 900/1100 zones (Figure 5d). To further understand whether the observed gradual increase of ALP activity in the different regions of the G Scaffolds could be related to a different cell density, ALP activity was also normalized by cell number/pore volume. Results confirmed an increased ALP activity with increasing pore size (Figure S5). 

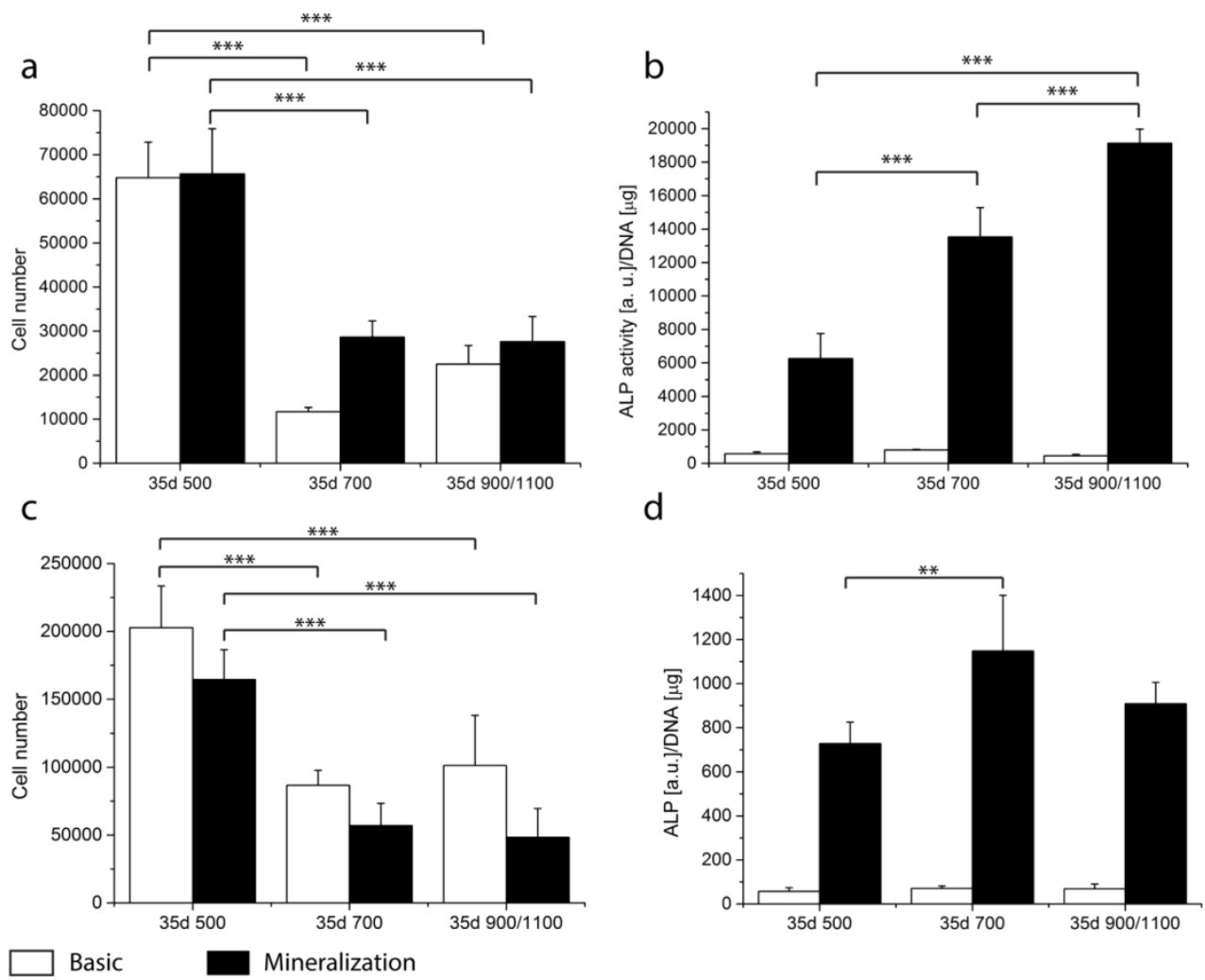

Figure 5. Cell number and ALP activity per gradient zone of donor $1 \mathrm{hMSCs}$ cultured on PCL scaffolds (a, b) and donor 2 on 300PEOT55PBT45 scaffolds (c, d). After 35 days the highest cell number is located in the area with the smallest pores. ALP activity displayed the same trend when donor 1 was cultured on PCL scaffolds, and a similar trend for donor 2 on 300PEOT55PBT45 scaffolds, with the lowest level in the 500 zone and the highest in 700 and $900 / 1100 .{ }^{* *}$ and ${ }^{* * *}$ statistical significance $p<0.01$ and $p<0.001$.

\subsection{ECM and mineralization analysis}

After 8 days in culture, pores were partially filled by ECM that looked fibrous in both culture conditions. After 35 days the amount of ECM increased and differentiated between the two conditions. Whereas in basic medium ECM kept having a dense fibrous appearance, in mineralization conditions some nodules were localized on

top of the dense fibrillar ECM structure. In order to confirm the possible mineralization of the ECM in correspondence to the observed nodules, an EDAX analysis was performed. In all the samples the mapping showed a co-localization 
of the colors representing both the calcium signal and the phosphate signal. The intensity of the two elements signal increased in correspondence of mineralization nodules. Qualitatively, nodules looked bigger in G and NG500 scaffolds compared to those found in the ECM of the NG1100 scaffolds (Figure 6).

G
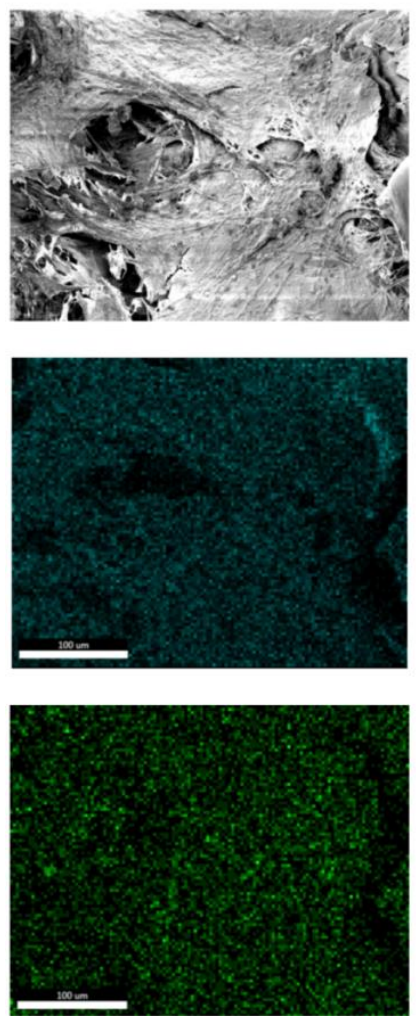

NG 500
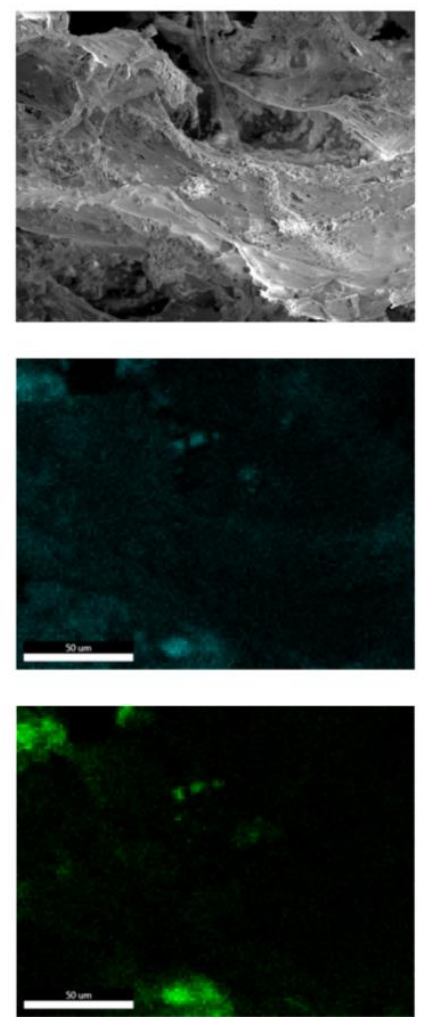

NG 1100
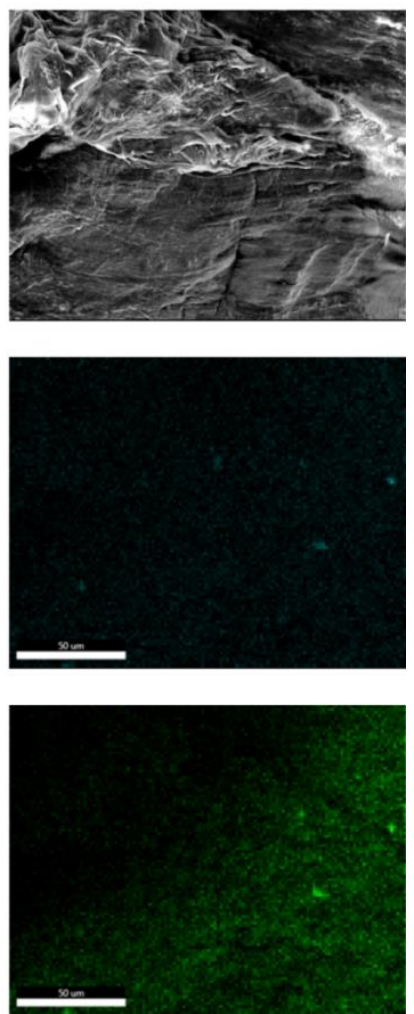

Figure 6. SEM micrograph and EDAX scan at day 35, revealing calcium (blue) and phosphate (green) for $\mathrm{G}$ and NG scaffolds. The co-localization of the colors suggested the presence of early ECM mineralization process. Scale bar $50 \mu \mathrm{m}$.

In order to quantify and confirm whether there were differences in the mineralization levels in the constructs, a calcium assay was performed. It was possible to observe an inverse trend in calcium content with respect to the porosity. Calcium levels were significantly higher in NG 500 compared to G and NG1100 scaffolds (Figure 7a). The partition analysis did not show any significant difference between the gradient zones (Figure 7b). However, when the scaffold volume was considered, NG1100 showed the highest calcium levels in full scaffolds. 

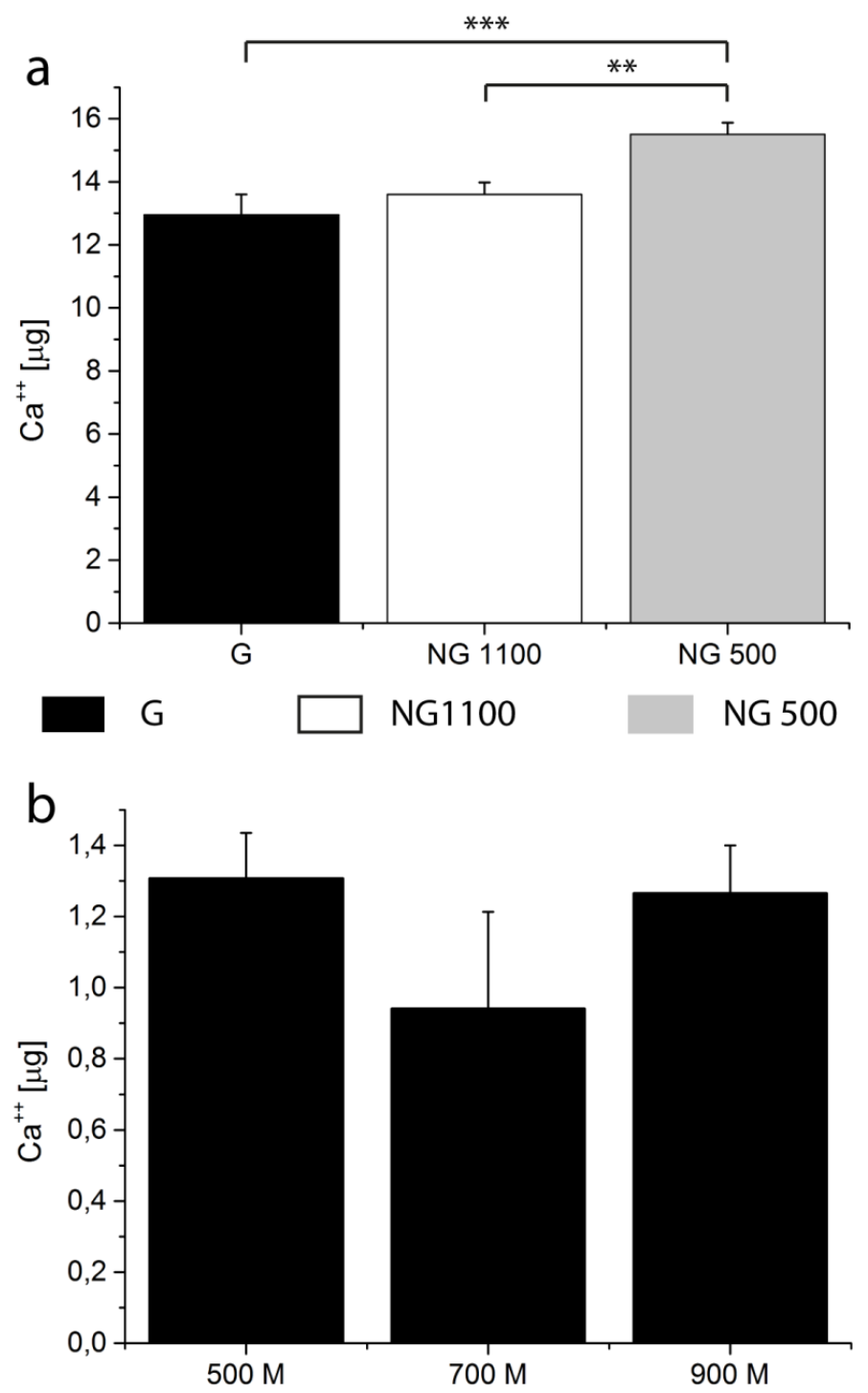

Figure 7. Calcium assay was performed on full scaffolds (a) as well as on partitioned gradient scaffolds (b) cultured under mineralization conditions for 28 days. Full scaffolds displayed an opposite trend in calcium content. The partition analysis did not show any significant difference among the gradient portions. ${ }^{* *}$ and ${ }^{* * *}$ statistical significance $p<0.01$ and $\mathrm{p}<0.001$ respectively.

Furthermore, when cell density was considered in the partition analysis, an increasing amount of calcium per cell density was measured with increasing pore size, thus confirming ALP results (Figure 8). To further dissect the role of cell 
density variations with varying pore size, we reasoned that differential availability of nutrients would be present in the different regions of gradient scaffolds. In particular, oxygen levels could decrease more rapidly with decreasing the pore size, thus leading more easily to higher hypoxic regions ${ }^{30}$. To prove this hypothesis, we measured hypoxia inducible factor (HIF)-1 $\alpha$ and HIF-2 $\alpha$. Results showed an increase of both HIFs with decreasing pore size (Figure S6).

\section{a Calcium Production per Scaffold Volume}

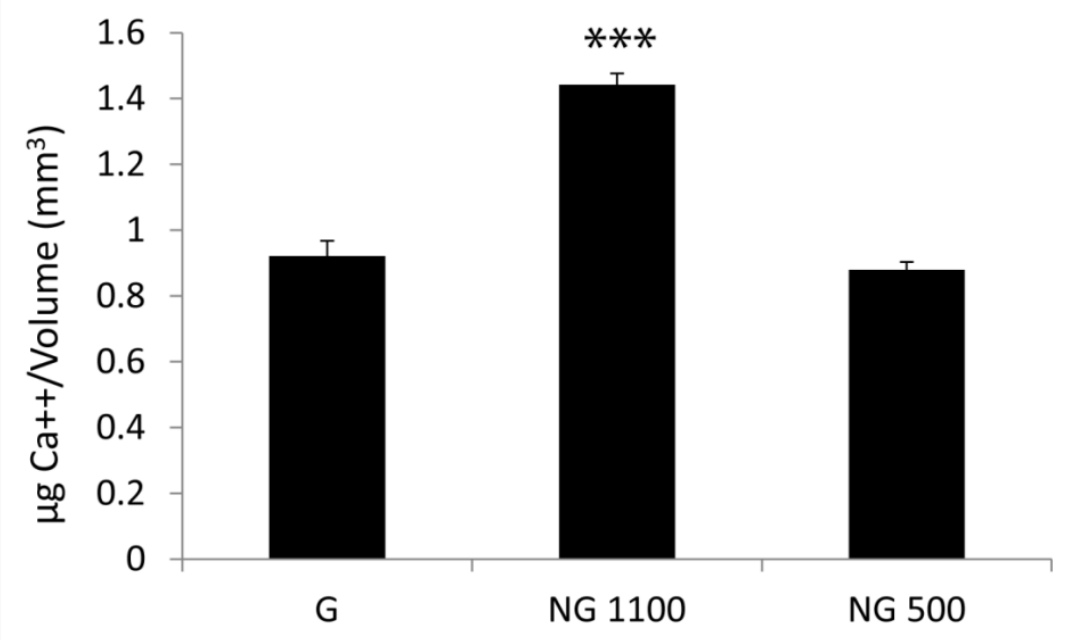

b Calcium Production per Pore Volume

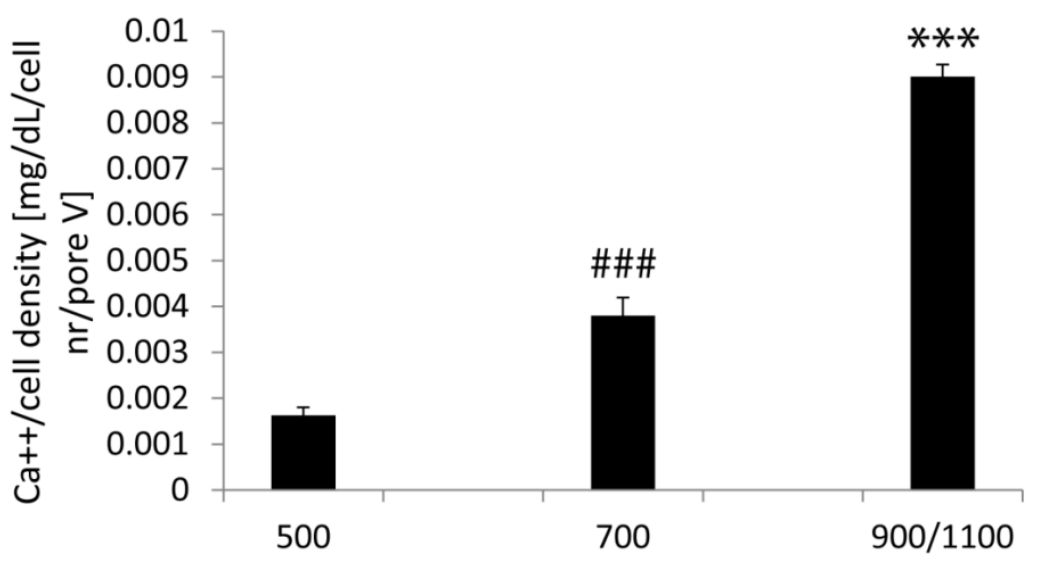

Figure 5. Calcium assay normalized by scaffold volume (a) as well as partition analysis of calcium levels normalized by cell density (b) cultured under mineralization conditions for 28 
days. ${ }^{* * *}$ shows statistical significance $p<0.001$ with respect to other scaffolds (a) or other regions. \#\#\# shows statistical significance $p<0.001$ compared to other gradient scaffold regions.

\section{Discussion}

Several strategies can be used to obtain a scaffold with a porosity gradient. Among them, fused deposition modeling emerged due to the possibility to fine tune several features such as fiber diameter, height and distance between the fibers. In the present work the fiber height (or layer thickness) was maintained constant and the fiber spacing was gradually increased along the $z$ axis. Scaffolds were homogeneously fabricated. The fiber size could experience small variations in the case of $G$ scaffolds due to the fact that with increasing the fiber spacing, and consequently the pore size, a larger distance has to be covered by the fibers before reaching the next contact point. This can create fiber size fluctuations due to the associated larger deformation of the molten polymer strand. Gradient scaffolds led to an axial gradient in pore size that affected the distribution and differentiation of hMSCs seeded in the resulting scaffolds. As already shown in previous studies, the design of the scaffolds and the pore geometry affect cell behavior in terms of adhesion, localization, ECM deposition and differentiation. Woodfield et al. proposed a 300PEOT55PBT45 gradient scaffolds for the zonal characterization of cartilage using primary chondrocytes ${ }^{31}$. More recently, Sobral et al. described cell adhesion and localization on gradient versus non gradient scaffolds, showing an improved cell seeding efficiency and distribution of an osteosarcoma cell line (SaOS) within the gradient scaffolds with respect to the non-gradient ones ${ }^{29}$. Despite what reported in literature, cell adhesion on the gradient and non-gradient scaffolds didn't show any significant difference in our study. This might be due to the different cell source used in our study, as it is known that hMSCs are more sensitive to environmental changes than already differentiated cells and cell lines. A difference between 300PEOT55PBT45 and PCL was observed, being the first one more favorable for cell adhesion, which might be due to a higher hydrophilicity (Figure S1). 
ALP is an enzyme responsible for the dephosphorylation of several molecules including nucleotides and proteins. ALP has always been related to osteoblast differentiation, as an increase of the enzyme activity was observed in the early stages of their commitment ${ }^{32}$. ALP activity in the gradient scaffolds was significantly higher after 28 days in mineralization medium in both donors compared to the non-gradient scaffolds. Despite the different levels of intensity, both donors showed a significantly higher ALP activity compared to the control in 300PEOT55PBT45 scaffolds. When presented as fold induction (Figure S3), the gradient increased ALP activity of the first donor by $25 \%$ and for the second donor by 2.5 times. The fold increase did not change when either NG500 or NG1100 were chosen as baseline. In case of PCL scaffolds, we could not observe a statistical difference between $G$ and NG scaffolds at a full scaffold level, despite higher ALP values for NG500 and G scaffolds (Figure 3d). This could be associated to the fact that $\mathrm{PCL}$ was shown to preferentially support chondrogenic more than osteogenic differentiation ${ }^{33}$. The partition analysis further highlighted the effect of the porosity on hMSC differentiation when they were cultured within the same constructs for both polymer chemistries, thus showing that also PCL supports a differential expression of ALP activity, which increased with increasing the pore size (Figures $4 \mathrm{~b}$ and 5 , and Figure $\mathrm{S} 4$ in supporting information). Therefore, further studies on additional biomaterial chemistries should be performed to conclude whether the influence of pore size gradients on hMSC osteogenic differentiation is specific for 300PEOT55PBT45 only or a more general phenomenon common to other biomaterials.

The cell number was significantly higher in the small pore zone at 8 and 35 days under basic and differentiation conditions. Comparing the cell number at 8 and 35 days it decreased in the $500 \mu \mathrm{m}$ zone and increased in the $700 \mu \mathrm{m}$ and 900/1100 $\mu \mathrm{m}$ zones. Since from the full analysis of the fabricated scaffolds it was shown that the cell number per scaffolds remained constant, a migration from the small pores toward the bigger ones can be hypothesized. Probably due to lower nutrient available determined by the higher number of cells and by the small pore size, hMSCs were stimulated to migrate toward pores with lower cell density and higher nutrient and oxygen availability ${ }^{34,35}$.The re-distribution of cells along a gradient in 
oxygen tension was shown by Ardakani et al..$^{36}$, thus corroborating our hypothesis. ALP activity showed an opposite trend with respect to the cell number. Although after 1 day in differentiation media (8 days in culture) no significant differences were seen between the areas of the gradient, after 28 days in differentiation media a gradient in ALP activity was generated having its maximum in the zone with the largest pores. Hsu et al. recently proved that under hypoxia condition hMSC differentiation toward the osteogenic lineage is impaired and the level of ALP expression attenuated ${ }^{30}$. A possible explanation could be found in the differences in pore size. Smaller pores closed faster, determining a longer lack of nutrient and oxygen availability for the cells residing in that zone with respect to the cells localized in the bigger pores areas. Thanks to the pore size change, the oxygen local concentration could have changed in the different areas of the $\mathrm{G}$ scaffolds. Therefore, more than cell density, oxygen distribution or pore size alone, these three factors seemed to be connected as a pore size increase resulted in a lower cell density, which consequently increased the local oxygen and nutrient availability. This could explain why the trend in ALP activity followed the direction of the gradient, which was further corroborated by a correspondent decrease of HIF$1 \alpha$ and HIF-2 $\alpha$ with increasing pore size. The molecular mechanism behind this phenomenon is still unclear and should be subject of further studies.

At the genetic level there were no major differences after 28 days of culture in relation to the influence of the analyzed structural scaffold gradient on hMSC differentiation. The increased expression of early genes such as ALP and BSP and, on the other hand, the missed upregulation of late markers such as OCN and OPN might suggest that the culture of hMSCs on these polymeric scaffolds didn't lead to a complete differentiation up to the later stages of mature osteoblasts. Despite the matrix mineralization followed a different trend compared to the ALP when looking at absolute calcium content, being the highest in the NG500 and the lowest in the NG1100 scaffolds, the same increasing trend with increasing pore size found for ALP was observed when taking into consideration cell density and pore volume.

Since the seeding procedure was performed by keeping the largest pores on top and the scaffold direction was not changed during culture, the gradient structure 
determined a higher localization of cells in the area with the small pores. To confirm that the seeding direction could play a role in the localization of the cells, the scaffolds were seeded and cultured in both ways. The dispersion of the cells within the scaffolds seemed to be influenced by its position at the moment of the seeding, since higher cell numbers were found in the area located at the bottom at the moment of the seeding (Figure S2). By comparing the two seeding direction, the ones presenting the highest cell number was still the one with the smallest pore size at the bottom. This area was the one presenting the highest surface available for cell attachment and growth. During culturing the cell number slightly decreased in the small pores zone and increased in the other zones (Figure $3 b$ ). This can be explained by 2 factors: i) the cells in the first week from the cells seeding were localized mainly in the area with the smaller pore size and evenly distributed in the remaining 3 zones; in the following 4 weeks, they expanded in number mainly in the bigger pore areas; ii) in the area with the smallest pore size, pore closure due to ECM deposition was faster. This might have affected the diffusion of oxygen and nutrients including the soluble factors present in the media. On the other hand, with increasing pore size cells have more room to grow and deposit the matrix. Filling of the pores with formed ECM took longer, ensuring a longer and better nutrient and oxygen diffusion. Our results showed an initial differentiation of hMSCs toward the osteogenic lineage. To clarify whether polymeric scaffolds are able to support a full differentiation in vitro, a longer study can be foreseen. Additionally, an animal study should be performed in order to define the effect of pore size in tissue formation in an ectopic location like a subcutaneous or intramuscular implantation. A further step should be the implantation of the gradient and non-gradient scaffolds in an orthotopic defect in a large animal models in order to study their performance in the healing of a critical defect.

\section{Conclusion}

A scaffold displaying a gradient in pore size along the $Z$ axis was plotted by varying the fiber spacing and its influence on hMSC differentiation was evaluated. The differentiation toward the osteogenic lineage of hMSCs cultured in mineralization 
media was improved in gradient scaffolds. The biomaterial used to fabricate the scaffolds did not seem to play a role, since PCL and 300PEOT55PBT45 showed the same trend when analyzing the local ALP activity in the different pore size gradient regions. Cells residing in different areas of the gradient displayed a trend in osteogenic differentiation following the pore size, probably due to a better supply of nutrient and oxygen in the compartments with the largest pores. Taken together the findings of this study introduce pore size gradients as a structural factor that could be taken into consideration when combining scaffolds and hMSCs for bone tissue engineering purposes.

\section{Materials \& Methods}

\subsection{Scaffolds preparation}

Scaffolds were fabricated via rapid prototyping (Bioscaffolder, SysENG, Germany). Scaffolds made of poly(ethylene oxide therephtalate)/poly(butylene therephtalate) (PEOT/PBT) and of poly( $\varepsilon$-caprolactone) (PCL) were produced. PEOT/PBT is a family of block co-polymers characterized by an aPEOTbPBTc nomenclature, where $a$ is the molecular weight of the starting PEG block and $b$ and $c$ are the PEOT/PBT ratio. Scaffolds made of both PCL and 300PEOT55PBT45 were already used in surgery and clinical trials are currently ongoing ${ }^{37}$. Briefly the polymers were placed in a stainless syringe and processed at $200{ }^{\circ} \mathrm{C}$ (300PEOT55PBT45, PolyVation, The Netherlands) and $100{ }^{\circ} \mathrm{C}$ (PCL, SigmaAldrich, USA). The molten polymer was extruded through a cartridge unit, by the application of a nitrogen flow with a pressure of 5 bar from a pressurized cap and an extrusion screw rotation of $200 \mathrm{rpm}$.

During plotting, the needle diameter, layer thickness and speed were kept constant at $200 \mu \mathrm{m}, 150 \mu \mathrm{m}$ and $180 \mathrm{~mm} / \mathrm{min}$, respectively. The fiber spacing was kept constant to $500 \mu \mathrm{m}$ and $1100 \mu \mathrm{m}$ for non-gradient (NG) scaffolds and varied from $500 \mu \mathrm{m}$ to $1100 \mu \mathrm{m}$ for gradient (G) scaffolds. The fiber spacing was changed 
every millimeter. The scaffolds were plotted in blocks of $20 \times 20 \mathrm{~mm}$ and $4 \mathrm{~mm}$ in height. The tested samples were $4 \times 4 \mathrm{~mm}$ cylinders punched out from the blocks.

\subsection{Cell expansion and culture}

Human mesenchymal stromal cells (hMSCs) were isolated from the bone marrow of donors with written informed consent ${ }^{38}$. Aspirates were resuspended using a $20 \mathrm{G}$ needle and plated at a density of 0.5 million mono-nucleated cells per $\mathrm{cm}^{2}$. Cells were grown in MSC proliferation medium, which contains minimal essential medium ( $\alpha$-MEM, Gibco, Breda the Netherlands) supplemented with $10 \%$ fetal bovine serum (FBS, Lonza), $100 \mathrm{U} / \mathrm{ml}$ penicillin (Gibco, breda the netherlands), 10 $\mu \mathrm{g} / \mathrm{ml}$ streptomycin (Gibco, USA), $2 \mathrm{mM} \mathrm{L-glutamin} \mathrm{(Gibco,} \mathrm{breda} \mathrm{the} \mathrm{netherlands),}$ $0.2 \mathrm{mM}$ L-ascorbic acid 2-phosphate magnesium salt (ASAp, Sigma-Aldrich, Zwijndrecht, The Netherlands) and $1 \mathrm{ng} / \mathrm{ml}$ of basic fibroblast growth factor-2 (bFGF-2, Fisher Scientific, Landsmeer, the Netherlands) at $37^{\circ} \mathrm{C}$ in a humid atmosphere with $5 \% \mathrm{CO}_{2}$. Cells were expanded up to approximately $80 \%$ confluency and either frozen for further use or seeded on the scaffolds.

\subsection{Cell seeding on scaffolds}

Two different hMSC donors were used in this study. D249 (D1, age 72, female) and D8004L (D2, age 22, male). For donor D249, bone marrow aspirates were obtained and experiments approved by the Medical Ethical committee of the local hospital (Dutch: Medisch Ethische Toetsingscommissie (MECT) van het Medisch Spectrum Twente) following the Dutch national ethics guidelines from patients who had given written informed consent. For donor D8004L, pre-selected hMSCs were retrieved from the Institute of Regenerative Medicine (Temple, Texas), which has supplied standardized preparations of MSCs to hundreds of laboratories under the auspices of an NIH/NCRR grant (P40 RR 17447-06). Human bone marrow aspirates were obtained under a protocol approved by an institutional review board. Briefly, a bone marrow aspirate was drawn and mononuclear cells were separated using density centrifugation. 
After trypsinization with $0.25 \%$ trypsin (Life Technologies, Bleiswijk, the Netherlands), cells (passage 2-4) were counted using a Bückner chamber and resuspended in proliferation media at a density of 500000 cells in $40 \mu \mathrm{L}$. The day before seeding, scaffolds were disinfected in $70 \% \mathrm{EtOH}$ for 30 min under stirring, washed 3 times in phosphate buffered saline solution (PBS) (Lonza, Breda, the Netherlands), and incubated overnight in cell proliferation media to allow protein adsorption on the scaffold's fibers. After protein adsorption, the $40 \mu \mathrm{L}$ of cell suspension were placed on the scaffolds in a drop wise fashion to account for a cell seeding density of 500000 cells/scaffold. The seeded scaffolds were placed for 4 hours in the incubator to allow cell adhesion before adding the cell culture medium.

Cells were cultured on the $G$ and NG scaffolds for 7 days in proliferation media. At day 7 , the proliferation media was changed and the cells within the scaffolds were cultured for another 1 and 28 days in basic medium, being the proliferation without the bFGF, and mineralization medium, consisting of basic medium supplemented with $10 \mathrm{nM}$ dexamethasone (Sigma-Aldrich, Zwijndrecht, The Netherlands) and 10 mM $\beta$-glycerol-phosphate (Sigma-Aldrich, Zwijndrecht, The Netherlands).

\subsection{Biochemical study}

\subsubsection{DNA analysis.}

The cell number per scaffold was calculated from the $\mu \mathrm{g}$ of DNA, obtained by a Cyquant DNA assay kit (Life Technologies, Bleiswijk, the Netherlands). Briefly, each scaffold was cut to improve lysis efficiency and freeze-thawed 5 times. After the freeze-thawing process, cells within the scaffolds were lysated by diluting the $20 x$ lysis buffer provided with the kit using a saline buffer $(180 \mathrm{mM} \mathrm{NaCl}, 1 \mathrm{mM}$ EDTA in distilled water). After $1 \mathrm{~h}$ of lysis, samples were sonicated 2 times for 10 seconds using a Branson sonifier 250 (Emerson Industrial Automation, USA). DNA content was quantified with a CyQuant kit (Invitrogen, Breda, the Netherlands) according to manufacturer's protocol and fluorescence was measured at $480 \mathrm{~nm}$ using a spectrophotometer LS50B (Perkin Elmer, The Netherlands). DNA concentrations were calculated from a $\lambda$ DNA standard curve. 


\subsubsection{ALP activity.}

To evaluate hMSC differentiation toward the osteogenic lineage, ALP content was measured using a CDP star kit (Roche, Woerden, The Netherlands). For this purpose, $10 \mu \mathrm{L}$ of sample was added to a well of a white 96 -well plate and $40 \mu \mathrm{L}$ of

substrate (Disodium 2-chloro-5-(4-methoxyspiro \{1,2-dioxetane-3,2'-(5'chloro)tricycle[3.3.1.13.7]decan\}-4-yl)-1-phenyl phosphate) was added. After 15 minutes incubation, luminescence was read using a spectrophotometer LS50B (Perkin Elmer). ALP activity was corrected for DNA content.

\subsubsection{Osteocalcin quantification.}

Osteocalcin (OCN) production was analyzed after 35 days of culture (7 days in basic medium followed by 28 days in mineralization medium) using ELISA (human osteocalcin ELISA kit, Invitrogen). The test was performed on 5 samples per condition. Briefly, the medium was removed and samples were washed using ice cold PBS. A buffer was prepared using 890 volumes of miliQ water, 100 volumes of RIPA buffer (Cell Technologies) and 10 volumes of Halt ${ }^{\mathrm{TM}}$ protease and phosphatase inhibitor (Thermo Scientific). 150 microliters of the buffer was added to each samples, which were incubated on ice for 10 minutes. The buffer was then collected from the samples and centrifuged at $11000 \mathrm{~g}$ for 15 minutes. The supernatant was collected and used for the ELISA tests which were performed according to the manufacturer's protocol. Briefly, $25 \mu \mathrm{l}$ of samples and standard solutions were added to the osteocalcin antibody-coated strip- well plates. $100 \mu \mathrm{l}$ of Anti-OST-HRP conjugate, respectively, were added to each correspondent well, and the plates were covered and incubated for 2 hours at room temperature. After incubation, the solutions in the wells were aspirated and the wells were washed 3 times using a washing solution provided in the kit. Then, $100 \mu \mathrm{l}$ of a chromogen solution (Tetramethylbenzidine) was added to each well and the plates were incubated for 30 minutes at room temperature in the dark. Finally, $100 \mu \mathrm{l}$ of stop solution was added to each well. The optical density of each well was read at 450 nm using a plate reader (MULTISKAN GO, Thermo Scientific). A standard curve 
was plotted in Microsoft excel and the concentration of OCN was determined in each well according to the standard curve.

\subsection{Gene expression analysis}

For gene expression analysis the scaffolds were taken from the medium washed twice with PBS, cut into small pieces and placed in an Eppendorf containing 750 $\mu \mathrm{L}$ of $\mathrm{TRIzo|} \circledast$ (Invitrogen) and stored at $-80^{\circ} \mathrm{C}$. In the case of partition analysis the gradient scaffolds were cut in order to separate the gradient zones and the 3 samples were located in the same vial prior the addition of the TRIzol $\AA_{\text {, in }}$ order to ensure the collection of enough RNA. RNA isolation was performed by using a Bioke RNA II nucleospin RNA isolation kit (Bioke, Leiden, The Netherlands). 150 $\mu \mathrm{L}$ of $\mathrm{CHCl}_{3}$ were added and the vials were vigorously mixed, followed by a centrifugation at $12000 \mathrm{~g}$ for 15 minutes at $4{ }^{\circ} \mathrm{C}$. The aqueous phase was transferred into a new tube and an equal amount of $70 \%$ ethanol was added. The mixture was transferred into a filter columns from the kit and the extraction was carried on by following the manufacturer's protocol. RNA concentration and purity was evaluated via an ND1000 spectrophotometer (Nanodrop Technologies, USA); cDNA was synthetized using iScript ${ }^{\mathrm{TM}}$ (BIO-RAD, Veenendaal, The Netherlands) according to manufacturer's protocol. Quantitative polymerase chain reaction (qPCR) was performed on the obtained cDNA by using the $\mathrm{iQ} \mathrm{SYBRBGree}$ Supermix (BIO-RAD, Veenendaal, The Netherlands) and the primers listed in Table 1. PCR reaction was carried out on the MyiQ2 Two-Color Real-Time PCR Detection System (BIO-RAD, Veenendaal, The Netherlands) under the following conditions, the cDNA was denatured for 10 minutes at $95{ }^{\circ} \mathrm{C}$, followed by 45 cycles, consisting of 15 seconds at $95^{\circ} \mathrm{C}, 15$ seconds at $60^{\circ} \mathrm{C}$ and 15 seconds at $72^{\circ} \mathrm{C}$. A melting curve was generated from each reaction to test the presence of primer dimers and aspecific products. The cycle threshold was calculated by the Bio-Rad iQ5 optical system software, in which the threshold was set in the lower log-linear region of the fluorescent signal. Ct values were normalized by the B2M housekeeping gene and $\Delta \mathrm{Ct}$ ((average of $\mathrm{Ct}$ control)-Ct value). Results were 
expressed as fold induction in mRNA expression normalized to the gene expression of the NG500 control scaffolds cultured in basic medium.

\begin{tabular}{|l|l|l|}
\hline Gene & Forward Primer & Reverse Primer \\
\hline B2M & ACAAAGTCACATGGTTCACA & GACTTGTCTTTCAGCAAGGA \\
\hline ALP & ACAAGCACTCCCACTTCATC & TTCAGCTCGTACTGCATGTC \\
\hline $\begin{array}{l}\text { Runx } \\
2\end{array}$ & TGGTTACTGTCATGGCGGGTA & TCTCAGATCGTTGAACCTTGCTA \\
\hline $\begin{array}{l}\text { Osteo } \\
\text { calcin }\end{array}$ & TGAGAGCCCTCACACTCCTC & CGCCTGGGTCTCTTCACTAC \\
\hline BSP & CCCCACCTTTTGGGAAAACCA & TCCCCGTTCTCACTTTCATAGAT \\
\hline OPN & CTCCATTGACTCGAACGACTC & CAGGTCTGCGAAACTTCTTAGAT \\
\hline
\end{tabular}

Table 1. Osteogenic markers used for the qPCR expressed as forward and reverse primer.

\subsection{Histological, computed tomography and SEM analysis}

$G$ and NG scaffolds were analyzed by scanning electron microscopy (SEM, Philips - XL 30 ESEM-FEG). Directly after plotting scaffold were punched, cut in half, gold sputtered and analyzed. SEM images were analyzed using Image $\mathrm{J}$ software in order to measure the fiber diameter, fiber spacing, and pore dimensions. Directly after plotting, scaffolds were also analyzed by computed tomography analysis using a source voltage and current of $40 \mathrm{kV}$ and $250 \mu \mathrm{A}$ respectively, 600 projections with an exposure of $100 \mathrm{~ms}$ and image pixel size of $3.97 \mu \mathrm{m}$.

Scaffolds cultured in mineralization medium for 28 days were fixed using $10 \%$ formalin, dehydrated by an increased series of ethanol concentration (50-60-70-8090-96-100\%) and cut in half. The final dehydration step was carried out using a Balzers CPD 030 Critical Point Drier.

Dry scaffolds were mounted on SEM stubs, gold sputtered (Cressington sputter coater 108 auto), and analyzed by Energy Dispersive X-Ray Analysis (EDAX, Ametek, USA). The picture of the area and the mapping of the localization of Calcium $(\mathrm{Ca})$ and phosphate $(\mathrm{P})$ element were acquired. 


\subsection{Calcium assay}

After 28 days in mineralization medium the scaffolds were collected, cut, sonicated and incubated in $1 \mathrm{M} \mathrm{HCl}$ solution for 3 days under agitation in order to release the Calcium. A QuantiChrom Calcium assay kit (DICA-500) (BioAssay System) was used to measure the calcium levels in each sample. The analysis was performed following the supplier protocol. Briefly, reagent $A$ and $B$ (provided with the kit) were mixed by combining equal volumes in order to obtain the working reagent. Five $\mu \mathrm{L}$ of each sample were pipetted in a clear 96 well plate and incubated for 3 minutes with $200 \mu \mathrm{L}$ of working reagent. Optical density was read at $612 \mathrm{~nm}$ using a Multiscan Go (Thermo Scientific, Breda, The Netherlands) plate reader.

\subsection{Partition analysis}

In order to analyze the behavior of hMSCs with the different pore sizes, at the end of the culture the gradient scaffolds were cut and the biochemical analysis was carried on the gradient zones separately. Due to the lower fiber number and the difficulty of separate them the areas with the biggest pores $(900 / 1100)$ were analyzed together.

\subsection{Statistical analysis}

All the quantitative data are expressed as mean \pm standard deviation. Statistics were performed using IBM SPSS Statistics 20. A two-way ANOVA with Tukey as post-hoc test were used. Differences between experimental groups were considered significant when $p \leq 0.05$.

\section{Acknowledgements}

Authors would like to acknowledge funding from the Dutch Technology Foundation (grant \# 11135). This research was also supported by the Province of Limburg and by the European Regional Development Fund within the Innovative Operational 
Program in the frame of project BIO-IMPLANT (Grant No. POIG.01.01.02-00022/09). 


\section{References}

1 Seidi, A., Ramalingam, M., Elloumi-Hannachi, I., Ostrovidov, S. \& Khademhosseini, A. Gradient biomaterials for soft-to-hard interface tissue engineering. Acta Biomater 7, 14411451, doi:10.1016/j.actbio.2011.01.011 (2011).

2 Melchels, F. P. W. et al. Additive manufacturing of tissues and organs. Progress in Polymer Science 37, 1079-1104, doi:10.1016/j.progpolymsci.2011.11.007 (2012).

3 Abdallah, B. M. \& Kassem, M. Human mesenchymal stem cells: from basic biology to clinical applications. Gene therapy 15, 109-116, doi:10.1038/sj.gt.3303067 (2008).

4 Marklein, R. A., Soranno, D. E. \& Burdick, J. A. Magnitude and presentation of mechanical signals influence adult stem cell behavior in 3-dimensional macroporous hydrogels. Soft Matter 8, 8113, doi:10.1039/c2sm25501d (2012).

5 Grayson, W. L., Zhao, F., Bunnell, B. \& Ma, T. Hypoxia enhances proliferation and tissue formation of human mesenchymal stem cells. Biochem Biophys Res Commun 358, 948-953, doi:10.1016/j.bbrc.2007.05.054 (2007).

6 Kawano, T., Sato, M., Yabu, H. \& Shimomura, M. Honeycomb-shaped surface topography induces differentiation of human mesenchymal stem cells (hMSCs): uniform porous polymer scaffolds prepared by the breath figure technique. Biomaterials Science 2, 52, doi:10.1039/c3bm60195a (2014).

$7 \quad$ Kasten, P. et al. Porosity and pore size of beta-tricalcium phosphate scaffold can influence protein production and osteogenic differentiation of human mesenchymal stem cells: an in vitro and in vivo study. Acta Biomater 4, 1904-1915, doi:10.1016/j.actbio.2008.05.017 (2008).

8 Justin R. Tse, A. J. E. Stiffness Gradients Mimicking In Vivo Tissue Variation Regulate Mesenchymal Stem Cell Fate. Plos One; DOI: 10.1371/journal.pone.0015978 (2011).

$9 \quad$ Chen, M. et al. Fabrication and characterization of a rapid prototyped tissue engineering scaffold with embedded multicomponent matrix for controlled drug release. International journal of nanomedicine 7, 4285-4297, doi:10.2147/IJN.S33083 (2012).

10 Lavery, K., Swain, P., Falb, D. \& Alaoui-Ismaili, M. H. BMP-2/4 and BMP-6/7 differentially utilize cell surface receptors to induce osteoblastic differentiation of human bone marrowderived mesenchymal stem cells. The Journal of biological chemistry 283, 20948-20958, doi:10.1074/jbc.M800850200 (2008).

11 Lin, S., Sangaj, N., Razafiarison, T., Zhang, C. \& Varghese, S. Influence of physical properties of biomaterials on cellular behavior. Pharm Res 28, 1422-1430, doi:10.1007/s11095-011-0378-9 (2011).

12 DANIĖLE NOËL, D. G., CELINE BOUQUET, FLORENCE APPARAILLY, CLAIRE BONY,, PASCALE PLENCE, V. M., GADI TURGEMAN, MICHEL PERRICAUDET, JACQUES SANY, \& JORGENSEN, C. Short-Term BMP-2 Expression Is Sufficient for In Vivo Osteochondral Differentiation of Mesenchymal Stem Cells 22, 74-85; (2004).

13 Hutmacher, D. W. Scaffolds in tissue engineering bone and cartilage. Biomaterials 21, 25292543; (2000).

14 Zein, I., Humacher, D.W., Tan, K.C., Teoh. S.H.. Fused deposition modeling of novel scaffold architectures for tissue engineering applications. Biomaterials 23, 1169-85, (2001).

15 Genzer, J. Templating Surfaces with Gradient Assemblies. The Journal of Adhesion 81, 417435, doi:10.1080/00218460590944855 (2005).

16 Bidan, C. M. et al. Geometry as a factor for tissue growth: towards shape optimization of tissue engineering scaffolds. Adv Healthc Mater 2, 186-194, doi:10.1002/adhm.201200159 (2013).

17 Moroni, L., de Wijn, J. R., van Blitterswijk, C. A.. Three-dimensional fiber-deposited PEOT/PBT copolymer scaffolds for tissue engineering: Influence of porosity, molecular network mesh size, and swelling in aqueous media on dynamic mechanical properties. $J$ Biomed Mater Res A, 75, 957-65; (2005).

18 Moroni, L., Poort, G., Van Keulen, F., de Wijn, J. R. \& van Blitterswijk, C. A. Dynamic mechanical properties of 3D fiber-deposited PEOT/PBT scaffolds: an experimental and numerical analysis. J Biomed Mater Res A 78, 605-614, doi:10.1002/jbm.a.30716 (2006).

19 Yeong, W. Y., Chua, C. K., Leong, K. F. \& Chandrasekaran, M. Rapid prototyping in tissue engineering: challenges and potential. Trends in biotechnology 22, 643-652 (2004). 
de Andrea, C. E. \& Hogendoorn, P. C. Epiphyseal growth plate and secondary peripheral chondrosarcoma: the neighbours matter. The Journal of pathology 226, 219-228, doi:10.1002/path.3003 (2012).

21 Zhou, S., Cui, Z. \& Urban, J. P. Factors influencing the oxygen concentration gradient from the synovial surface of articular cartilage to the cartilage-bone interface: a modeling study. Arthritis and rheumatism 50, 3915-3924, doi:10.1002/art.20675 (2004).

Wang, Q., Wang, Q. \& Wan, C. Preparation and evaluation of a biomimetic scaffold with porosity gradients in vitro. Anais da Academia Brasileira de Ciencias 84, 9-16 (2012).

Giannoudis, P. V., Chris Arts, J. J., Schmidmaier, G. \& Larsson, S. What should be the characteristics of the ideal bone graft substitute? Injury 42, S1-S2, doi: 10.1016/j.injury.2011.06.001 (2011).

Giannoudis, P. V., Dinopoulos, H. \& Tsiridis, E. Bone substitutes: An update. Injury 36, S20S27, doi: 10.1016/j.injury.2005.07.029 (2005).

Dupont, S. et al. Role of YAP/TAZ in mechanotransduction. Nature 474, 179-183, doi:10.1038/nature10137 (2011).

$\mathrm{Wu}$, J. et al. Gradient biomaterials and their influences on cell migration. Interface focus $\mathbf{2}$, 337-355, doi:10.1098/rsfs.2011.0124 (2012).

Wang, X. et al. Growth factor gradients via microsphere delivery in biopolymer scaffolds for osteochondral tissue engineering. Journal of Controlled Release 134, 81-90, doi: 10.1016/j.jconrel.2008.10.021 (2009).

Singh, M. et al. Three-dimensional macroscopic scaffolds with a gradient in stiffness for functional regeneration of interfacial tissues. J Biomed Mater Res A 94, 870-876, doi:10.1002/jbm.a.32765 (2010).

Sobral, J. M., Caridade, S. G., Sousa, R. A., Mano, J. F. \& Reis, R. L. Three-dimensional plotted scaffolds with controlled pore size gradients: Effect of scaffold geometry on mechanical performance and cell seeding efficiency. Acta Biomater 7, 1009-1018, doi:10.1016/j.actbio.2010.11.003 (2011).

Hsu, S. H., Chen, C. T. \& Wei, Y. H. Inhibitory effects of hypoxia on metabolic switch and osteogenic differentiation of human mesenchymal stem cells. Stem cells (Dayton, Ohio) 31, 2779-2788, doi:10.1002/stem.1441 (2013).

Woodfield, T. B. et al. Polymer scaffolds fabricated with pore-size gradients as a model for studying the zonal organization within tissue-engineered cartilage constructs. Tissue Eng 11, 1297-1311 (2005).

32 Siffert, R. S. The role of alkaline phosphatase in osteogenesis. The Journal of experimental medicine 93, 415-426 (1951).

33 Chastain, S. R., Kundu, A. K., Dhar, S., Calvert, J. W. \& Putnam, A. J. Adhesion of mesenchymal stem cells to polymer scaffolds occurs via distinct ECM ligands and controls their osteogenic differentiation. Journal of biomedical materials research 78, 73-85 (2006).

Ju, S. Y. et al. Effect of hypoxic treatment on bone marrow cells that are able to migrate to the injured liver. Cell biology international 33, 31-35, doi:10.1016/j.cellbi.2008.10.002 (2009).

indothelial cells triggers NO production, cell migration, and angiogenesis. Journal of cellular physiology 221, 359-366, doi:10.1002/jcp.21861 (2009).

36 Ardakani, A. G., Cheema, U., Brown, R. A. \& Shipley, R. J. Quantifying the correlation between spatially defined oxygen gradients and cell fate in an engineered three-dimensional culture model. Journal of the Royal Society, Interface / the Royal Society 11, 20140501, doi:10.1098/rsif.2014.0501 (2014).

37 Bartha, L. et al. A clinical feasibility study to evaluate the safety and efficacy of PEOT/PBT implants for human donor site filling during mosaicplasty. European journal of orthopaedic surgery \& traumatology : orthopedie traumatologie 23, 81-91, doi:10.1007/s00590-011-0907-6 (2013).

38 Both, S. K., van der Muijsenberg, A. J., van Blitterswijk, C. A., de Boer, J. \& de Bruijn, J. D. A rapid and efficient method for expansion of human mesenchymal stem cells. Tissue Eng 13, 3-9, doi:10.1089/ten.2005.0513 (2007). 


\section{SUPPORTING INFORMATION}

\begin{tabular}{|c|c|c|c|}
\hline Fiber spacing $[\mu \mathrm{m}]$ & Porosity [\%] & Pore size $[\mu \mathrm{m}]$ & $\begin{array}{c}\text { Pore volume } \\
{\left[\mathrm{mm}^{3}\right]}\end{array}$ \\
\hline 500 & $58 \%$ & $270 \pm 10$ & $0.015 \pm 0.002$ \\
\hline 700 & $70 \%$ & $500 \pm 30$ & $0.055 \pm 0.008$ \\
\hline 900 & $77 \%$ & $710 \pm 20$ & $0.098 \pm 0.009$ \\
\hline 1100 & $81 \%$ & $870 \pm 50$ & $0.181 \pm 0.026$ \\
\hline
\end{tabular}

Table S1. Table displaying the pore volume per gradient zone. The average volume increased by 10 times from the smallest to the largest pore size area.

\begin{tabular}{|c|c|}
\hline Sample & Porosity [\%] \\
\hline G & $67.83 \pm 3.3$ \\
\hline NG 500 & $47.24 \pm 6.9$ \\
\hline NG 1100 & $80.63 \pm 2.3$ \\
\hline
\end{tabular}

Table S2. Table displaying the porosity of the full scaffolds. As expected the porosity of the $\mathrm{G}$ scaffolds were in between the porosity of the controls.

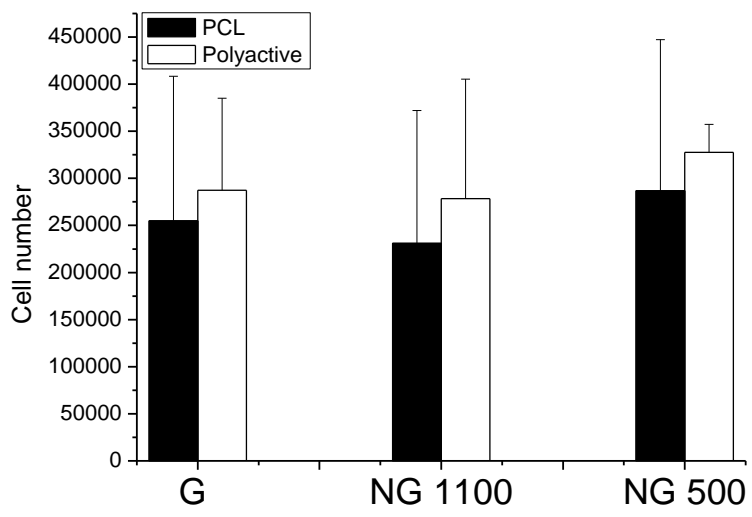

Figure S1. Cell seeding efficiency of 300PEOT55PBT45 after 8 hours. The two materials showed a similar cell seeding efficiency. 


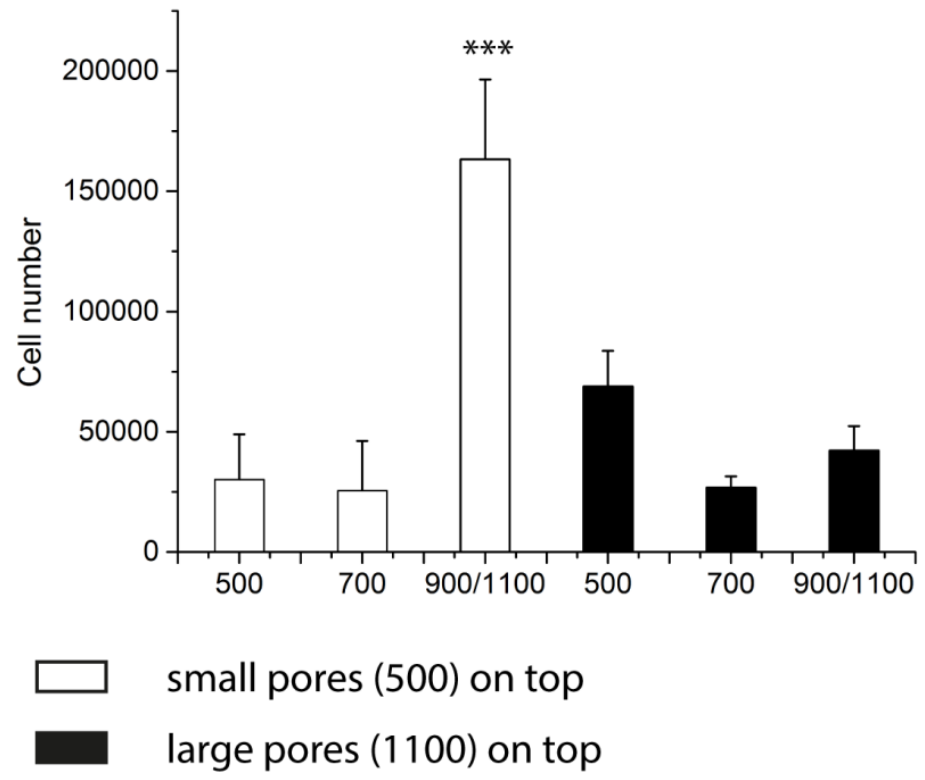

Figure S2. Plot showing the cell number per gradient zone related to the seeding direction. The highest number of cells is located in the area that at the seeding moment is located at the bottom. 

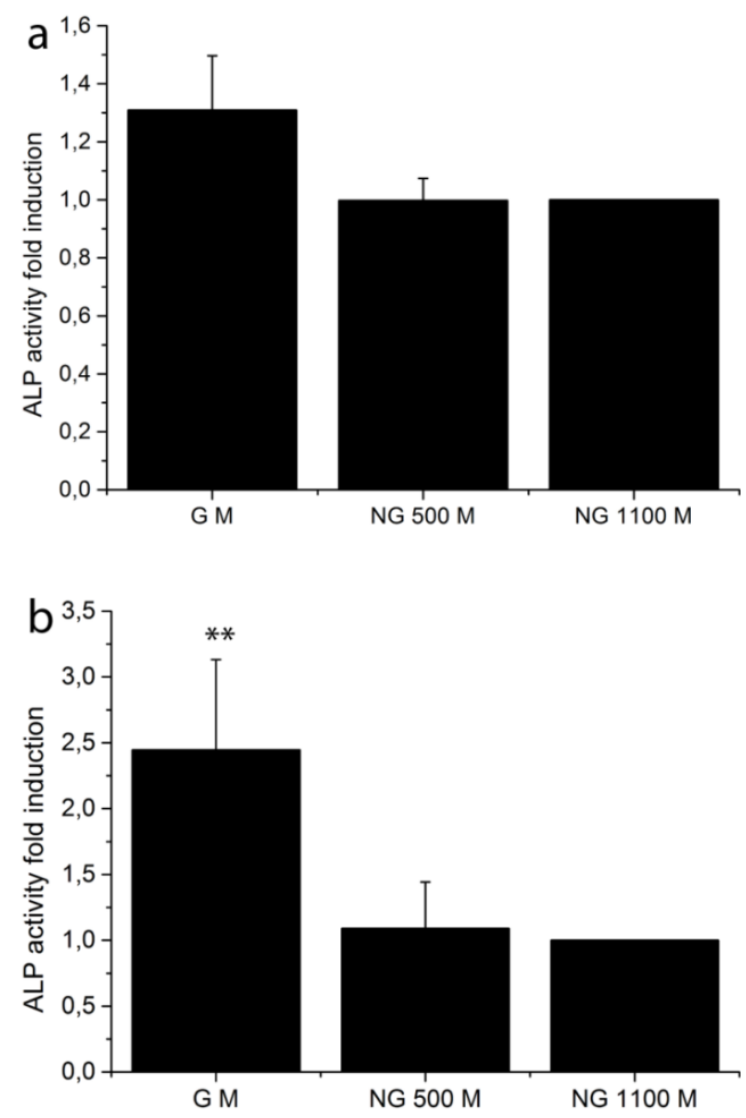

Figure S3. ALP activity of D1 (a) and D2 (b) expressed as fold induction normalized by the ALP activity levels of NG1100. In D1 the ALP activity was increased by $25 \%$ and significantly increased by 2.5 times in D2. A normalization by NG500 gave the same fold induction. ${ }^{* *}$ statistical significance $p<0.01$. 


\section{Osteocalcin Production per Scaffold Volume - 28 days}

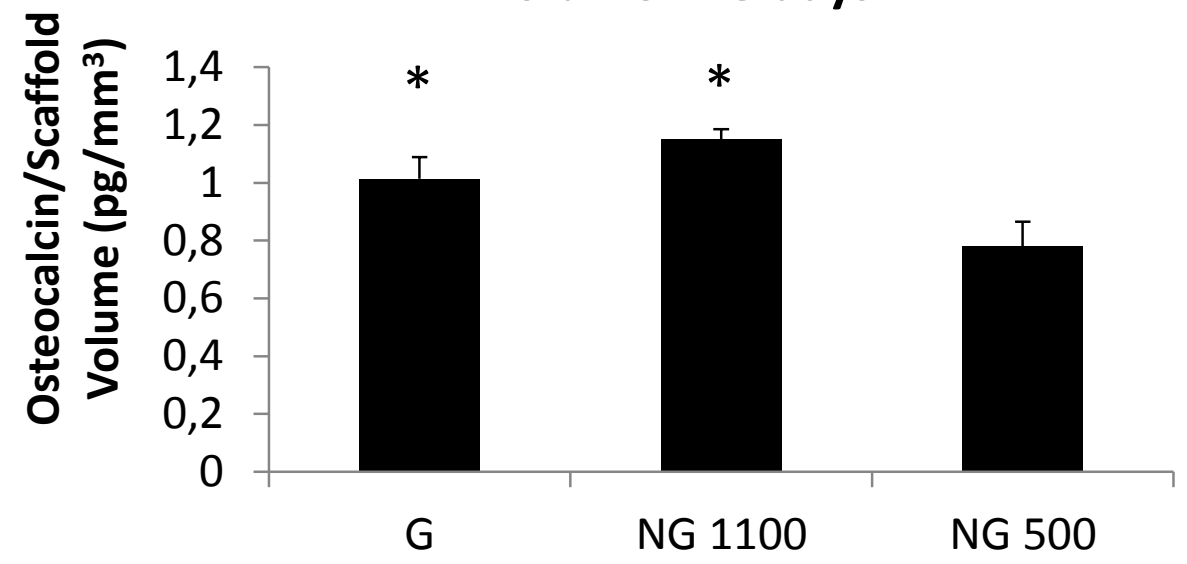

Figure S4. Osteocalcin production measured by ELISA assay on G, NG 1100 and NG 500 scaffolds show significantly higher amounts of osteocalcin in G and NG 1100 scaffolds, compared to NG 500 scaffolds. ${ }^{*}$ indicates statistical significance with $p<0.05$.
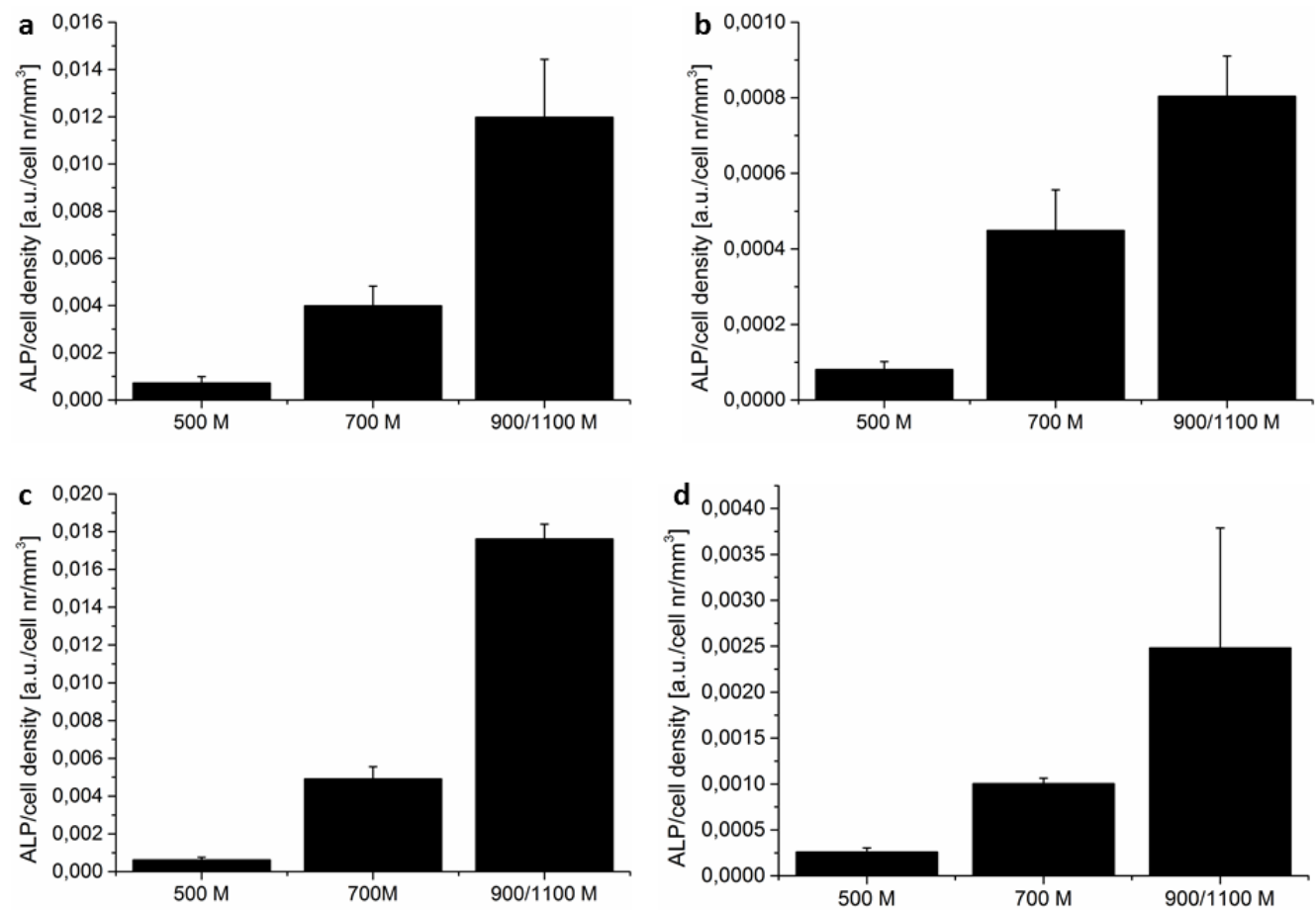
Figure S5. Partition analysis of ALP activity of D1 (a, c) and D2 (b, d) in 300PEOT55PBT (a, b) and PCL (c, d) gradient scaffolds normalized by cell density.
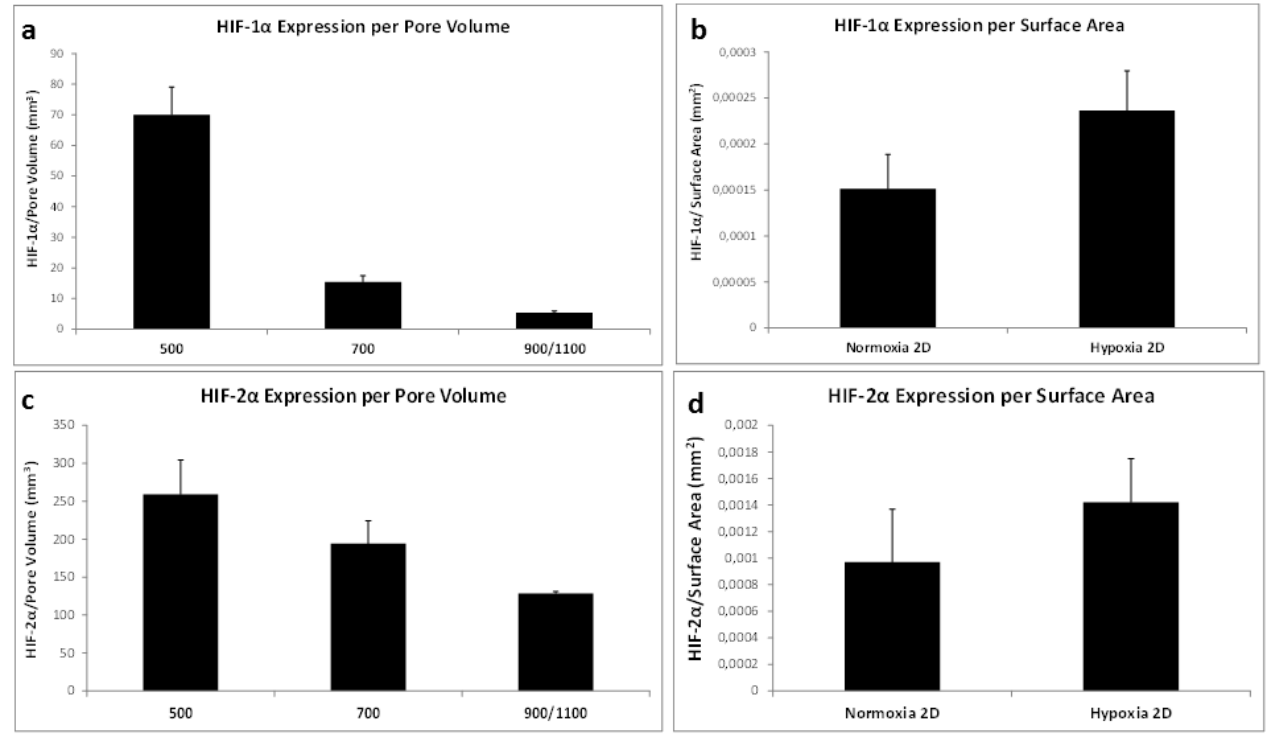

Figure S6. HIF-1 $\alpha$ (a) and HIF-2 $\alpha$ (c) expression in the different regions of gradient scaffolds, showing a decrease of HIF factors with increasing the pore size. (b, d) control experiments in 2D cell culture plates in normoxic and hypoxic conditions 


\section{CHAPTER 3}

\section{Influencing chondrogenic differentiation of human mesenchymal stromal cells in scaffolds displaying a structural gradient in pore size}

Andrea Di Luca, Karol Szlazak, Ivan Lorenzo-Moldero, Corina A. Ghebes, Antonio

Lepedda, Wojcech Swieszkowski, Clemens Van Blitterswijk, Lorenzo Moroni 


\begin{abstract}
Articular cartilage lesions have a limited ability to heal by themselves. Yet, golden standard treatments for cartilage repair such as drilling, microfracture and mosaicplasty provide further damage and an unstable solution that degenerates into fibrocartilage in time. Articular cartilage presents a number of gradients in cell number and size along with structural gradients in extra cellular matrix (ECM) composition. Therefore, creating scaffolds that display a structural gradient can be an appealing strategy for cartilage tissue regeneration treatments. In the present study, a scaffold with an in-built discrete gradient in pore size was produced by additive manufacturing. Human mesenchymal stromal cells (hMSCs) were seeded within the gradient scaffolds and their proliferation, differentiation and ECM deposition was evaluated with respect to 2 non-gradient scaffolds. Glycosaminoglycan (GAG) deposition was significantly higher in gradient scaffolds and non-gradient scaffolds with the smallest pore size compared to non-gradient scaffolds with the largest pore size. A gradual increase of chondrogenic markers was observed within the gradient structures with decreasing pore size, which was also accompanied by an increasingly compact ECM formation. Therefore, scaffolds displaying a structural gradient in pore size seem to be a promising strategy to aid in the process of hMSC chondrogenic differentiation and could be considered for improved cartilage tissue regeneration applications.
\end{abstract}




\section{Introduction}

Cartilage is the tissue located at the extremity of long bones and is responsible for the transmission of forces from the articular surface to the underlying subchondral bone [1]. Cartilage can be affected by progressive degenerative diseases such as osteoarthritis, which involves the osteochondral or chondral tissue depending on the depth of the injury. This process can be triggered by a severe trauma, repetitive minor injuries or aging process [2]. Currently, an optimum treatment for such type of lesions is still under debate. Articular cartilage lesions generally do not heal, or heal only partially under certain biological conditions [3]. Among the available treatments, most of them are based on the disruption of the damaged site such as drilling, microfracture or spongialization $[4,5]$. Bleeding from the subchondral bone would then ensure the delivery of mononuclear cells and growth factors contained in the bone marrow responsible for the temporary healing of the damaged site. Other proposed techniques consist in the transplantation of autologous cells [6, 7], perichondrial/periosteal grafts [8], and autologous or allogenic osteochondral transplantation $[9,10]$. Since all these proposed solutions present several drawbacks related to morbidity, risks of infections, lack of available tissues, and redegeneration of the repaired tissue, the use of biomaterial-based strategies to promote and direct tissue growth emerged in the past decades. Several techniques have been proposed in literature to produce scaffolds, such as freeze drying [11], salt leaching [12, 13], solvent casting [13] and gas foaming [14]. The success of a scaffold resides in some characteristic features such as total porosity, pore size and interconnectivity, mechanical and physico-chemical properties. The fore mentioned techniques lack control of one or more of these characteristics. Additive manufacturing has been used to fabricate scaffolds due to its ability of overcoming these drawbacks and the possibility to fine tune scaffolds characteristics such as pore shape and size. Additionally, a gradual variation of the scaffolds characteristics can be achieved within the same construct, allowing the design and production of scaffolds displaying structural gradients [15, 16]. Gradients are also present in the human body from the developmental phase to adult life. Morphogen gradients are known to lead to the formation of the osteochondral tissue [17]. At 
the tissue interface, the gradual variation from one tissue to the other is based on gradients. Skin and bone present a gradient structure in the axial direction and the radial direction, respectively. Cartilage is a tissue which display an internal variation of properties such as stiffness [18-20], extracellular matrix (ECM) composition [2, 18] and cell shape and number [21, 22] along the axial direction as well. In particular, articular cartilage display a gradient in proteoglycans, collagen type II, and water content. While proteoglycan content increases from the calcified region to the surface plateau, collagen type II and water content increases from the surface plateau to the calcified zone [23]. Therefore, the use of gradient scaffolds could be a feasible solution for cartilage regeneration. In the present study, scaffolds with a discrete gradient in pore size in the axial direction were fabricated by additive manufacturing. The proliferation, ECM deposition and differentiation of human bone marrow derived mesenchymal stromal cells (hMSCs) toward the chondrogenic lineage were evaluated. Furthermore, we analyzed the differentiation of hMSCs within the compartments of the gradient in order to correlate their differentiation to the size of the pores. We hypothesized that a variation in pore size could indirectly result into a local gradient in nutrient availability during culture. Correspondently, this may result in gradual hypoxic culture conditions, which are known to facilitate chondrogenesis [24, 25]. hMSCs have been chosen due to their potential to differentiate into different skeletal lineages, among others [26, 27]. Their presence in the mononuclear cell fraction of the subchondral bone marrow further guarantees a potentially straightforward clinical application, as hMSCs are often thought to be responsible for cartilage repair in current clinical surgical procedures such as microfracture.

\section{Materials \& Methods}

\subsection{Scaffolds preparation}

Scaffolds were fabricated via 3D fiber deposition (Bioscaffolder, SysENG, Germany). Scaffolds made of poly(ethylene oxide therephtalate)/poly(butylene 
therephtalate) (PEOT/PBT) were produced. PEOT/PBT is a family of block copolymers characterized by an aPEOTbPBTc nomenclature, where $a$ is the molecular weight of the starting PEG block and $b$ and $c$ are the PEOT/PBT ratio. A 300PEOT55PBT 45 composition was chosen due to its proven potential for skeletal regeneration [28, 29]. Briefly, the polymer (300PEOT55PBT45, PolyVation, The Netherlands) was placed in a stainless syringe and processed at $200{ }^{\circ} \mathrm{C}$. The molten polymer was extruded through a cartridge unit, by the application of a nitrogen flow with a pressure of 5 bar from a pressurized cap and an extrusion screw rotation of $200 \mathrm{rpm}$.

During plotting, the needle diameter, layer thickness and speed were kept constant at $200 \mu \mathrm{m}, 150 \mu \mathrm{m}$ and $180 \mathrm{~mm} / \mathrm{min}$, respectively. The fiber spacing was kept constant to $500 \mu \mathrm{m}$ and $1100 \mu \mathrm{m}$ for non-gradient (NG) scaffolds and varied from $500 \mu \mathrm{m}$ to $1100 \mu \mathrm{m}$ for gradient (G) scaffolds. The fiber spacing was changed every millimeter. The scaffolds were plotted in blocks of $20 \times 20 \mathrm{~mm}$ and $4 \mathrm{~mm}$ in height. The tested samples were $4 \times 4 \mathrm{~mm}$ cylinders punched out from the blocks.

\subsection{Cell expansion and culture}

hMSCs (male, age 22) were retrieved from the Institute of Regenerative Medicine (Temple, Texas). Briefly, a bone marrow aspirate was drawn and mononuclear cells were separated using density centrifugation. The cells were plated to obtain adherent hMSCs, which were harvested when cells reached $60-80 \%$ confluence. These were considered passage 0 (P0) cells. These P0 cells were expanded, harvested and frozen at passage 1 (P1) for distribution. Cells were grown in MSC proliferation medium, which contains minimal essential medium ( $\alpha-M E M$, Life Technologies, Bleiswijk, the Netherlands) supplemented with $10 \%$ fetal bovine serum (FBS, Lonza), $100 \mathrm{U} / \mathrm{ml}$ penicillin (Life Technologies, Bleiswijk, the Netherlands), $10 \mu \mathrm{g} / \mathrm{ml}$ streptomycin (Life Technologies, Bleiswijk, the Netherlands), $2 \mathrm{mM}$ L-glutamin (Life Technologies, Bleiswijk, the Netherlands), 0.2 $\mathrm{mM}$ L-ascorbic acid 2-phosphate magnesium salt (ASAp, Sigma-Aldrich, Zwijndrecht, The Netherlands) and $1 \mathrm{ng} / \mathrm{ml}$ of basic fibroblast growth factor-2 (bFGF-2, Fisher Scientific, Landsmeer, the Netherlands) at $37^{\circ} \mathrm{C}$ in a humid 
atmosphere with $5 \% \mathrm{CO}_{2}$. Cells were expanded up to approximately $80 \%$ confluency and either frozen for further use or seeded on the scaffolds.

\subsection{Cell seeding on scaffolds}

After trypsinizaition with $0.25 \%$ trypsin (Life Technologies, Bleiswijk, the Netherlands), cells (passage 2-4) were counted using a Bückner chamber and resuspended in proliferation medium at a density of 500'000 cells in $40 \mu \mathrm{L}$. The day before seeding, scaffolds were disinfected in $70 \% \mathrm{EtOH}$ for 30 min under stirring, washed 3 times in phosphate buffered saline solution (PBS) (Lonza, Breda, the Netherlands), and incubated overnight in proliferation medium to allow protein adsorption on the scaffold's fibers. After protein adsorption, the $40 \mu \mathrm{L}$ of cell suspension were placed on the scaffold in a drop wise fashion to account for a cell seeding density of 500'000 cells/scaffold. The seeded scaffolds were placed for 4 hours in the incubator to allow cell adhesion before adding the cell culture medium. Cells were cultured on the $G$ and NG scaffolds for 7 days in proliferation medium. At day 7 , the proliferation medium was changed and the cell-seeded scaffolds were cultured for another 28 days in basic medium presenting the same formulation as the proliferation medium without bFGF, or chondrogenic medium consisting of DMEM supplemented with $50 \mathrm{mg} / \mathrm{mL}$ ITS-premix (Bexton Dickinson), $0.4 \mathrm{mM}$ Proline (Sigma-Aldrich, Zwijndrecht, The Netherlands), $50 \mathrm{mg} / \mathrm{mL}$ ascorbic acid (ASAp, Sigma-Aldrich, Zwijndrecht, The Netherlands), $100 \mathrm{mg} / \mathrm{mL}$ sodium pyruvate (Sigma-Aldrich, Zwijndrecht, The Netherlands), $100 \mathrm{U} / \mathrm{ml}$ penicillin (Life Technologies, Bleiswijk, the Netherlands), $10 \mu \mathrm{g} / \mathrm{ml}$ streptomycin (Life Technologies, Bleiswijk, the Netherlands), $10 \mathrm{ng} / \mathrm{mL}$ transforming growth factor $\beta 3$ (TGF- $\beta 3$ ) (Life Technologies, Bleiswijk, the Netherlands) and $10^{-7} \mathrm{M}$ dexamethasone (Sigma-Aldrich, Zwijndrecht, The Netherlands). Scaffolds were flipped every day so to exchange "bottom" and "top" side of the scaffolds to take into account the possible effect of gravity on cell distribution, where the "top" side is considered as the one with the smaller pore size in the gradient scaffolds. 


\subsection{Partition analysis}

To see the differences within the hMSCs cultured in the different area of the gradient scaffolds, after 14 and 28 days under differentiation conditions, the constructs were collected, washed 3 times with PBS and cut in 3 portions of $1 \mathrm{~mm}$ in height. The division of the pore size 900 with the pore size 1100 was not possible due to the poor mechanical properties; therefore, the 2 pore size portions were analyzed together. Biochemical studies and gene expression analysis were performed to the full and partitioned scaffolds.

\subsection{Biochemical study}

\subsubsection{DNA analysis.}

The cell number per scaffold was calculated from the $\mu \mathrm{g}$ of DNA, obtained by a Cyquant DNA assay kit (Life Technologies, Bleiswijk, the Netherlands). Briefly, each scaffold was cut to improve lysis efficiency and freeze-thawed 5 times. After the freeze-thawing process, cells within the scaffolds were lysated by diluting the $20 \mathrm{x}$ lysis buffer provided with the kit using a saline buffer $(180 \mathrm{mM} \mathrm{NaCl}, 1 \mathrm{mM}$ EDTA in distilled water). After $1 \mathrm{~h}$ of lysis, samples were sonicated 2 times for 10 seconds using a Branson sonifier 250 (Emerson Industrial Automation, USA). DNA content was quantified with a CyQuant kit (Life Technologies, Bleiswijk, the Netherlands) according to manufacturer's protocol and fluorescence was measured at $480 \mathrm{~nm}$ using a spectrophotometer LS50B (Perkin Elmer, The Netherlands). DNA concentrations were calculated from a $\lambda$ DNA standard curve.

\subsubsection{GAG amount}

To evaluate the differentiation toward the chondrogenic lineage, the GAG amount was quantified using 1,9-Dimethyl Methylene Blue (DMMB) assay. Therefore, 25 $\mu \mathrm{L}$ of sample were placed into a transparent flat bottom 96 well plate and $5 \mu \mathrm{L}$ of 2.3M NaCl solution were added, then $150 \mu \mathrm{L}$ of DMMB solution were added and absorbance was read using a Multiscan Go (Fisher Scientific, Landsmeer, the 
Netherlands) plate reader at a wavelength of $525 \mathrm{~nm}$. GAG content was quantified with a chondroitin standard curve and corrected for DNA content.

\subsubsection{Western Blot Analysis}

hMSC passage 2-4 were cultured in normoxic (21\% oxygen) conditions on both petri dishes and 3D scaffolds, and in hypoxic (2,5\% oxygen) conditions on petri dishes only (positive control). The cells were lysed in radio-immunoprecipitation assay (RIPA) lysis buffer (sc-24948, SantaCruz Biotechnology). The protein content was determined using a Pierce BCA protein assay kit (\#23227, Thermo Scientific Pierce), with bovine serum albumin used as a standard. Each sample was mixed with 4x Laemmli Sample Buffer (\#161-0737, BioRad) under denaturing conditions. $20 \mu \mathrm{g}$ of protein sample were loaded per lane and resolved on a $4-20 \%$ precast polyacrylamide gel (\#456-8094, BioRad). Proteins were transferred to a PVDF membrane for immunoblotting. After blocking $1 \mathrm{~h}$ at room temperature with $5 \%$ BSA in Tris-buffered saline containing 0.1\% Tween-20 (Santa Cruz Biotechnology), the membranes were incubated overnight at $4^{\circ} \mathrm{C}$ with HIF-1 $\alpha$ (28b) antibody (\#sc-13515, Santa Cruz Biotechnology), or HIF-2a antibody (\#sc-46691, Santa Cruz Biotechnology), diluted 1:250 in Tris-buffered saline containing 0.1\% Tween-20). GAPDH antibody (\#sc-365062, Santa Cruz Biotechnologies) diluted 1:2500 was used as loading control for normalization. Membranes were washed with Tris-buffered saline, and incubated with horseradish peroxidase (HRP)conjugated secondary antibodies (DAKO) diluted 1:2500 in Tris-buffered saline containing $0.1 \%$ Tween-20 for $45 \mathrm{~min}$ at room temperature. After washing, blots were developed using Clarity Western ECL Substrate (\#1705060, BioRad) and the HRP activity of the blots were imaged using a BioRad Chemidoc Touch imager. Finally, blots were semi-quantified using ImageJ.

\subsection{SEM analysis and computed tomography analysis}

$G$ and NG scaffolds were analyzed by scanning electron microscopy (SEM, Philips - XL 30 ESEM-FEG). Directly after plotting, scaffolds were punched, cut in half, gold sputtered (Cressington sputter coater 108 auto) and analyzed. SEM images 
were analyzed using Image $\mathrm{J}$ software in order to measure the fiber diameter, fiber spacing, and pore dimensions. Pore dimensions can be calculated by subtracting the fiber dimension from the fiber spacing in the $x-y$ plane, while they are identical to the layer thickness in the $z$ direction [30]. Scaffolds cultured in basic and chondrogenic media for 28 days were fixed using $10 \%$ formalin, dehydrated by an increasing series of ethanol concentration (50-60-70-80-90-96-100\%) and cut in half. The final dehydration step was carried out via immersion in Hexamethyldisilazane (Sigma-Aldrich, Zwijndrecht, The Netherlands) and overnight evaporation. Dry scaffolds were mounted on SEM stubs, gold sputtered, and analyzed for tissue formation. Directly after plotting, scaffolds were analyzed by micro-computed tomography $(\mu \mathrm{CT})$. The $\mathrm{X}$-ray measurements were performed using SkyScan 1172 (Bruker) with the following working parameters of X-ray tube: $40 \mathrm{kV}$ and $250 \mu \mathrm{A}$. For a $3.97 \mu \mathrm{m}$ pixel size, 600 projections were taken with exposure time of $100 \mathrm{~ms}$ and 5 integrations for each exposition. The projections were reconstructed with the special software (NRecon, ver. 1.6.10.4, Bruker microCT) and then exported to CTAn (ver. 1.15.4.0+, Bruker microCT) for further 3D image analysis. Reconstructed slices were processed, resulting in 3D binary images. Object volume, available surface and fiber spacing were calculated for the whole volume of analyzed scaffolds.

\subsection{Gene expression analysis}

For gene expression analysis, the scaffolds were taken from the medium, washed twice with PBS, cut into small pieces, placed in an Eppendorf containing $750 \mu \mathrm{L}$ of TRIzol ${ }^{8}$ (Invitrogen) and stored at $-80^{\circ} \mathrm{C}$. In case of partition analysis, the gradient scaffolds were cut in order to separate the gradient zones and the 3 samples were located in the same vial prior the addition of TRIzol $\Theta$, in order to ensure the collection of enough RNA. RNA isolation was performed by using a Bioke RNA II nucleospin RNA isolation kit (Bioke, Leiden, The Netherlands). $150 \mu \mathrm{L}$ of $\mathrm{CHCl}_{3}$ were added and the vials were vigorously mixed, followed by a centrifugation at $12000 \mathrm{~g}$ for 15 minutes at $4 \stackrel{\circ}{\circ}$. The aqueous phase was transferred into a new tube and an equal amount of $70 \%$ ethanol was added. The mixture was transferred 
into a filter column from the kit and the extraction was carried on following the manufacturer's protocol. RNA concentration and purity was evaluated via an ND1000 spectrophotometer (Nanodrop Technologies, USA); cDNA was synthetized using iScript ${ }^{\mathrm{TM}}$ (BIO-RAD, Veenendaal, The Netherlands) according to the manufacturer's protocol. Quantitative polymerase chain reaction (qPCR) was performed on the obtained $c D N A$ by using the $i Q$ SYBR $B$ Green Supermix (BIORAD, Veenendaal, The Netherlands) and the primers listed in Table 1. PCR reaction was carried out on a MyiQ2 Two-Color Real-Time PCR Detection System (BIO-RAD, Veenendaal, The Netherlands) under the following conditions: the $\mathrm{CDNa}$ was denatured for 10 minutes at $95 \stackrel{\circ}{\circ}$, followed by 45 cycles, consisting of 15

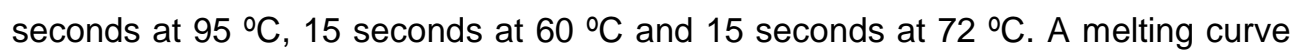
was generated from each reaction to test the presence of primer dimers and unspecific products. The cycle threshold was calculated by the Bio-Rad iQ5 optical system software, in which the threshold was set in the lower log-linear region of the fluorescent signal. Ct values were normalized by the B2M housekeeping gene and $\triangle \mathrm{Ct}$ ((average of $\mathrm{Ct}$ control)-Ct value). Results were expressed as fold induction in mRNA expression normalized to the gene expression of the gradient scaffolds cultured in basic medium. In the partition analysis, the relative RNA expression was normalized by the big pore region (900/1100) cultured under chondrogenic conditions.

\begin{tabular}{|c|c|c|}
\hline Gene & Forward Primer & Reverse Primer \\
\hline B2M & ACAAAGTCACATGGTTCACA & GACTTGTCTTTCAGCAAGGA \\
\hline Aggrecan & AGGCAGCGTGATCCTTACC & GGCCTCTCCAGTCTCATTCTC \\
\hline Sox9 & TGGGCAAGCTCTGGAGACTTC & ATCCGGGTGGTCCTTCTTGTG \\
\hline Collagen & CGTCCAGATGACCTTCCTACG & TGAGCAGGGCCTTCTTGAG \\
\hline Ila & & \\
\hline
\end{tabular}

Table 1. List of primers used for the gene expression anlysis of hMSC differentiation toward the chondrogenic lineage. 


\subsection{Statistical analysis}

All the quantitative data are expressed as mean \pm standard deviation. Statistics were performed using IBM SPSS Statistics 20. A two-way ANOVA with Tukey as post-hoc test was used. Statistical significance between the control group and the experimental groups is indicated with $\left({ }^{*}\right)$ which represents a $p$-value < $0.05,\left({ }^{* *}\right)$ which represents a $p$-value $<0.01$, and $\left(^{* * *}\right)$ which represents a $p$ value $<0.001$

\section{Results}

\subsection{Scaffold characterization}

Four zones in the gradient scaffold can be distinguished (Figure 1a and 1d), where the fiber spacing changed from bottom to top from $463 \mu \mathrm{m} \pm 17 \mu \mathrm{m}$, to $646 \mu \mathrm{m} \pm 9$ $\mu \mathrm{m}, 836 \mu \mathrm{m} \pm 23 \mu \mathrm{m}, 1023 \mu \mathrm{m} \pm 26 \mu \mathrm{m}$. The resulting pore size was $326 \mu \mathrm{m} \pm 17$ $\mu \mathrm{m}$ when the fiber spacing was set at $500 \mu \mathrm{m}, 540 \mu \mathrm{m} \pm 11 \mu \mathrm{m}$ when the fiber spacing was set at $700 \mu \mathrm{m}, 744 \mu \mathrm{m} \pm 16 \mu \mathrm{m}$ and $968 \mu \mathrm{m} \pm 25 \mu \mathrm{m}$ when the fiber spacing was set at 900 and $1100 \mu \mathrm{m}$ respectively as analyzed from SEM. The fiber diameter was measured as $155 \pm 20 \mu \mathrm{m}$. The $\mu \mathrm{CT}$ analysis showed a pore size of $237.67 \mu \mathrm{m} \pm 7.77 \mu \mathrm{m}$ when the fiber spacing was set at $500 \mu \mathrm{m}, 389.33 \mu \mathrm{m} \pm 4.93$ $\mu \mathrm{m}$ with a fiber spacing of $700 \mu \mathrm{m}, 509 \mu \mathrm{m} \pm 4.36 \mu \mathrm{m}$ and $538.33 \mu \mathrm{m} \pm 50.64 \mu \mathrm{m}$ when the fiber spacing was at $900 \mu \mathrm{m}$ and $1100 \mu \mathrm{m}$ respectively (Table 2b). Control scaffolds were plotted by keeping the fiber spacing constant at $500 \mu \mathrm{m}$ and $1100 \mu \mathrm{m}$, which was measured as $458 \mu \mathrm{m} \pm 15 \mu \mathrm{m}$ and $939 \mu \mathrm{m} \pm 88 \mu \mathrm{m}$, respectively, by SEM (Figure $1 \mathrm{c}$, and $\mathrm{d}$ ). By increasing the fiber spacing, the volume of the pore in the different zones correspondently increased by $50 \%$ from the smallest to the largest pore size. (Figure 1e). In parallel, by increasing the pore size the surface available decreased from $114.75 \mathrm{~mm}^{2} \pm 11.34 \mathrm{~mm}^{2}$ in the smaller pore area to $44.72 \mathrm{~mm}^{2} \pm 14.16 \mathrm{~mm}^{2}$ in the largest pore area (Table 2b). As expected, the overall porosity of the gradient scaffolds was in between the porosity of the controls (Figure 1f). The porosity of the different gradient areas increased from $57 \% \pm 0.07 \%$ close to the porosity of the NG $500(47.24 \% \pm 6.9 \%)$, to $80 \% \pm$ 
$0.04 \%$ that matched with porosity values of NG 1100 (80.63\% $\pm 2.3 \%$ ). The porosity calculated with the $\mu \mathrm{CT}$ showed that the porosity of $\mathrm{G}$ scaffolds were close to the one of NG500 scaffolds, $69.52 \% \pm 0.31 \%$ and $70.80 \% \pm 0.06 \%$. NG1100 scaffolds displayed a porosity of $78.88 \% \pm 0.7 \%$ (Table $2 a$ ).

a

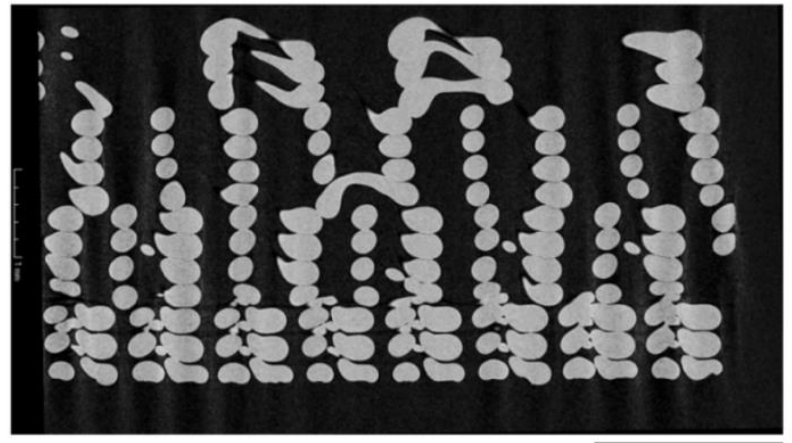

b

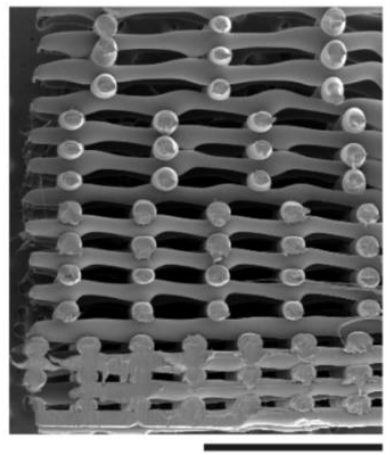

C

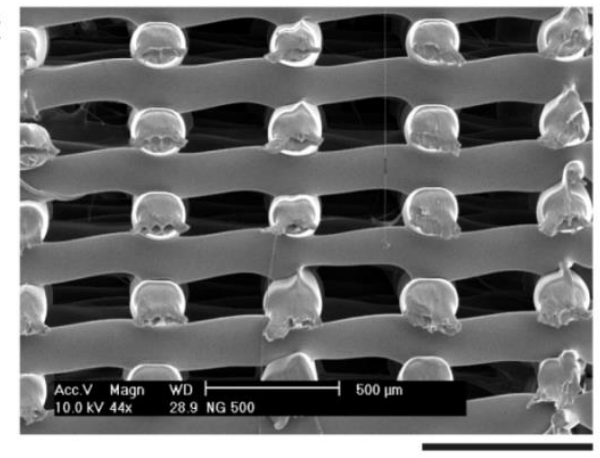

d

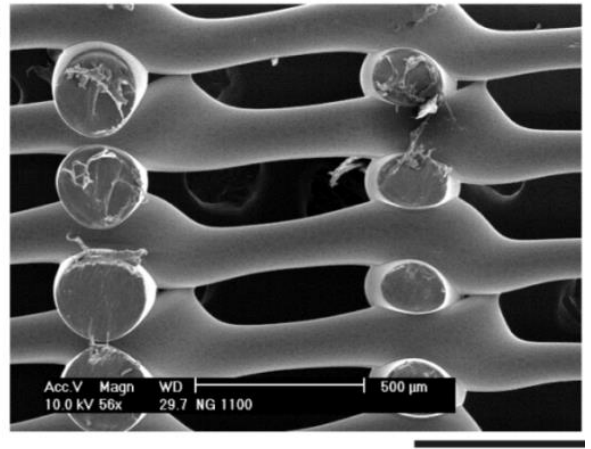

e

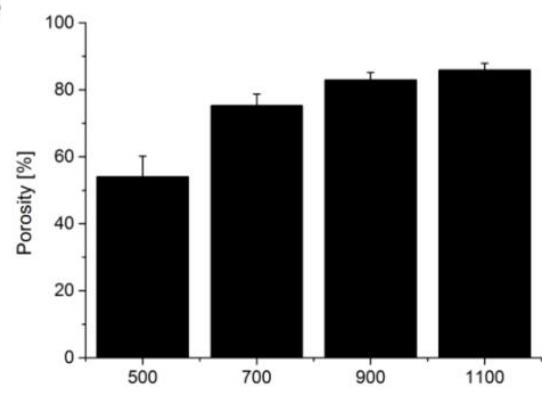

f

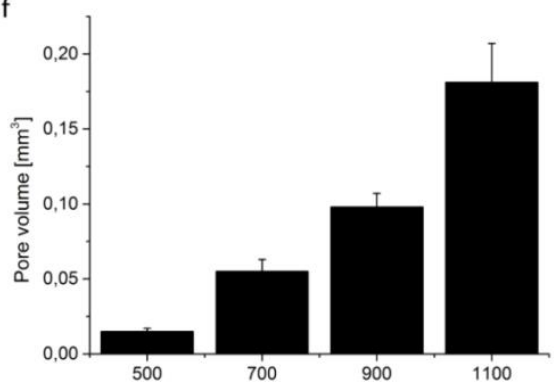

Figure 1. Micrograph of the gradient and control scaffolds; a - b) $\mu \mathrm{CT}$ reconstruction and SEM pictures of the $G$ scaffold cross section. Pore size is increasing with the fiber spacing along the $z$ axis (scale bar $2 \mathrm{~mm}$ ), SEM micrograph of the control scaffolds NG500 (c) and 
NG1100 (d) (scale bar $500 \mu \mathrm{m}$ ). Graph displaying the increase in porosity (e) and pore volume $(f)$ along the $z$ axis in the gradient scaffold.

\begin{tabular}{|c|c|c|c|}
\hline$A$ & $\begin{array}{l}\equiv=5 \\
\text { En }\end{array}$ & $\frac{253}{3-3}$ & $\begin{array}{l}=1 \equiv \\
\text { NG1100 }\end{array}$ \\
\hline $\begin{array}{l}\text { Objectiv } \\
\text { e } \\
\text { volume } \\
\left(\mathrm{mm}^{3}\right)\end{array}$ & $14.06 \pm 2.79$ & $15.47 \pm 0.01$ & $10.75 \pm 0.98$ \\
\hline $\begin{array}{l}\text { Availabl } \\
\text { e } \\
\text { surface } \\
\left(\mathrm{mm}^{2}\right)\end{array}$ & $292.91 \pm 16.57$ & $366.82 \pm 1.94$ & $198.57 \pm 19.42$ \\
\hline $\begin{array}{l}\text { Porosity } \\
(\%)\end{array}$ & $69.52 \pm 0.31$ & $70.80 \pm 0.06$ & $78.88 \pm 0.70$ \\
\hline $\begin{array}{l}\text { Structur } \\
\mathrm{e} \\
\text { thicknes } \\
\mathrm{s}(\mu \mathrm{m})\end{array}$ & $218.42 \pm 1.64$ & $164.04 \pm 0.39$ & $235.54 \pm 2.75$ \\
\hline $\begin{array}{l}\text { Averag } \\
\text { e pore } \\
\text { size } \\
(\mu \mathrm{m})\end{array}$ & $464 \pm 14.7$ & $303.5 \pm 0.7$ & $650.3 \pm 55.1$ \\
\hline
\end{tabular}

\begin{tabular}{|c|c|c|c|c|c|}
\hline b GRADIENT & $\begin{array}{c}\text { Fiber } \\
\text { spacing }\end{array}$ & $\begin{array}{l}\text { Pore } \\
\text { size } \\
(\mu \mathrm{m})\end{array}$ & $\begin{array}{c}\text { Porosity } \\
(\%)\end{array}$ & $\begin{array}{c}\text { Available } \\
\text { surface } \\
\left(\mathrm{mm}^{2}\right)\end{array}$ & $\begin{array}{c}\text { Pore } \\
\text { volume } \\
\left(\mathrm{mm}^{3}\right)\end{array}$ \\
\hline$: \div: 8$ & 1100 & $\begin{array}{l}538.3 \pm \\
50.6\end{array}$ & $\begin{array}{l}81.8 \pm \\
0.8\end{array}$ & $44.7 \pm 14.2$ & $\begin{array}{c}8.1 \pm \\
0.4\end{array}$ \\
\hline $8: 8:$ & 900 & $\begin{array}{c}509 \pm \\
4.36\end{array}$ & $\begin{array}{l}76.0 \pm \\
0.7\end{array}$ & $59.8 \pm 7.6$ & $\begin{array}{l}7.2 \pm \\
0.2\end{array}$ \\
\hline$E \equiv \equiv$ & 700 & $\begin{array}{l}389.3 \pm \\
4.9\end{array}$ & $\begin{array}{l}69.6 \pm \\
1.5\end{array}$ & $73.6 \pm 6.0$ & $\begin{array}{c}6.6 \pm \\
0.1\end{array}$ \\
\hline 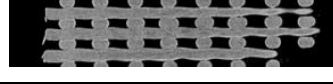 & 500 & $\begin{array}{l}237.7 \pm \\
7.8\end{array}$ & $\begin{array}{c}55.1 \pm \\
1.4\end{array}$ & $\begin{array}{c}114.7 \pm \\
11.3\end{array}$ & $\begin{array}{l}5.5 \pm \\
0.3\end{array}$ \\
\hline
\end{tabular}

Table 2. Table depicting the structural properties of the entire scaffolds (a) and of the different zones of the gradient scaffolds (b). All the scaffold properties of the gradient 
scaffolds, with exception of the porosity, are between the values of the controls. Along the $z$ axis there is an increase in pore size from the 500 to the 1100 zone and a parallel decrease of the available surface.

\subsection{Cell seeding efficiency and cell number}

The presence of the gradient as well as the different pore size of the controls didn't affect cell attachment at 8 hours from seeding (Fig. 2a). At 28 days of differentiation, scaffolds cultured in basic conditions displayed a higher cell number compared to the ones cultured under chondrogenic conditions (Fig. 2b). Even at this late time point the porosity didn't affected the cell number per structure, since there was no statistical difference among gradient and non-gradient scaffolds.
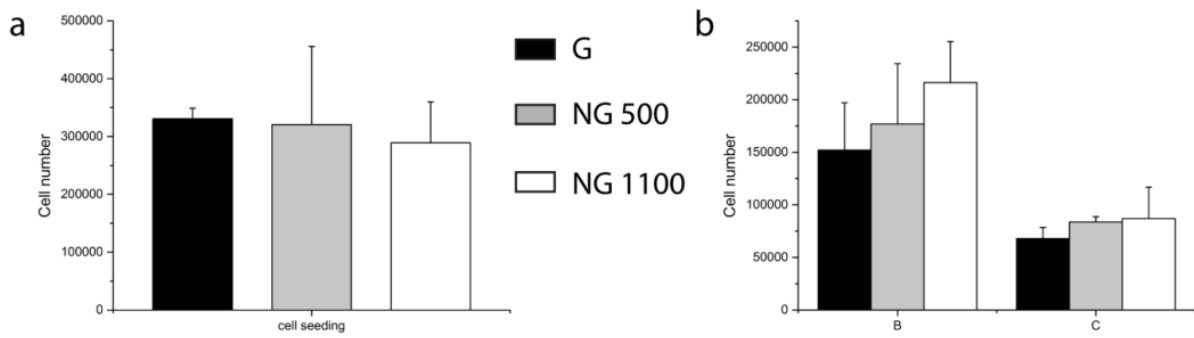

Figure 26. Graphs displaying the cell seeding efficiency of each construct after 8 hours (a) and the cell number per construct after 28 days of culture under basic and chondrogenic conditions (b).

\subsection{Induction of chondrogenic markers}

After 14 days under chondrogenic conditions 2 out of 3 markers chosen to detect chondrogenesis were upregulated (Fig. 3a). The early marker sox9 appeared highly upregulated with a 7-fold induction for the gradient and a 6- and 8-fold induction for the NG500 and NG1100, respectively. The late marker aggrecan was even more upregulated with a 38-fold induction for the gradient, and a 32- and 31fold induction for NG500 and NG1100 respectively. Collagen type II displayed an initial increase in its expression with an upregulation of 2 to 3 times fold. No major differences could be detected among the gradient and non-gradient structures. After 28 days under differentiation conditions the produced glycosaminoglycan 
(GAG) displayed a trend in which the NG1100 scaffolds showed $13.08 \pm 4.98 \mu \mathrm{g}$ of GAG/DNA, which was significantly lower than the amount of GAG deposited by NG500 scaffolds ( $26.2 \pm 2.07 \mu \mathrm{g}$ of GAG/DNA) as shown in Figure $3 \mathrm{~b}$. G scaffolds showed an amount of $G A G / D N A$ of $21.39 \pm 2.92$, which was significantly higher with respect to the amount produced in NG1100 scaffolds.

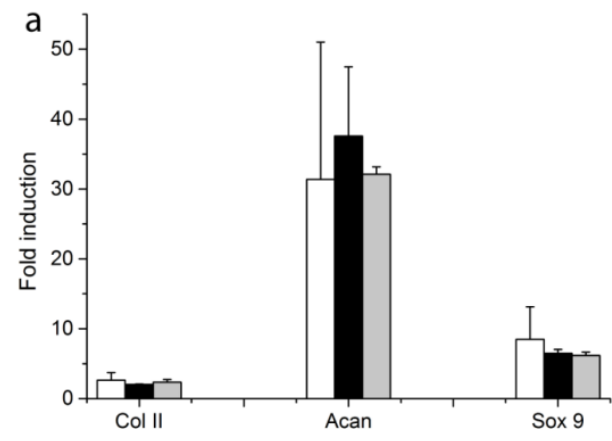

m $\mathrm{G}$

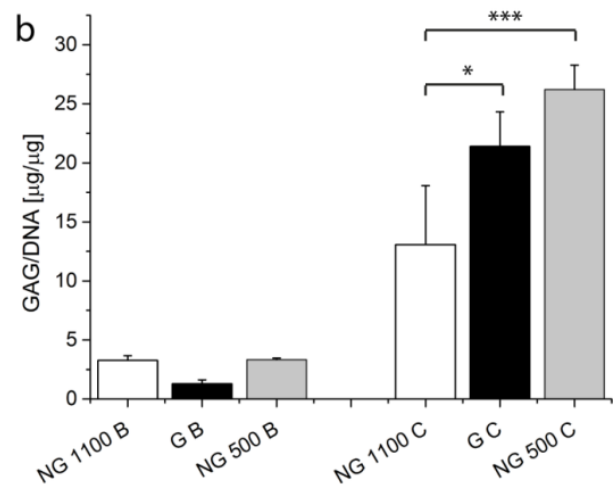

NG 500

NG 1100

Figure 3. Chondrogenic differentiation at genic level after 14 days (a) and in GAG deposited after 28 days (b) by hMSCs residing in $\mathrm{G}$ and NG scaffolds. The expression in chondrogenic markers did not show any statistical difference among the scaffolds. Small pores and the gradient displayed a beneficial effect on chondrogenic differentiation with respect to bigger pores. As expected the culture under chondrogenic conditions significantly enhanced the GAG/DNA amount with respect to the culture in basic media. * Statistical significance $p<$ 0,05 and ${ }^{* * *} p<0,001$.

\subsection{ECM analysis}

After 28 days, the constructs cultured in basic medium displayed cells adhering to the fibers with produced ECM bridging the pores and connecting subsequent fibers (Figure 4 a-f). When cultured under chondrogenic conditions, all the samples displayed the presence of ECM closing most of the pores (Fig $4 \mathrm{~g}-\mathrm{I}$ ). Samples cultured under chondrogenic conditions displayed a consistently higher ECM.

As shown in Figure 4 (m-p) from the small pores to the big pores throughout the entire pore network, the gradient scaffolds were filled with ECM. The ECM organization seemed more compact in the area with fiber spacing of $700 \mu \mathrm{m}$ and $500 \mu \mathrm{m}$ (Figure $4 \mathrm{n}, \mathrm{o}$ ), and became looser when moving to the bottom part of the scaffolds characterized by the higher fiber spacing (Figure 4 p). Higher 
magnification showed in both $G$ and $N G$ scaffolds spread cell morphology, yet with more ECM fibrillary bundles present in G scaffolds (Supporting Information, Figure S2).

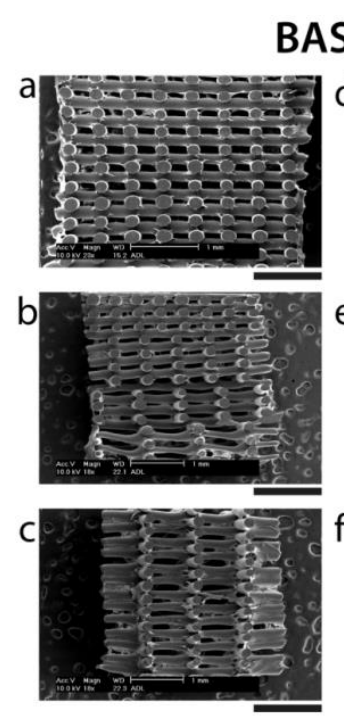

G

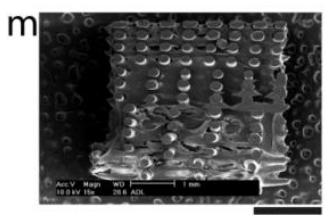

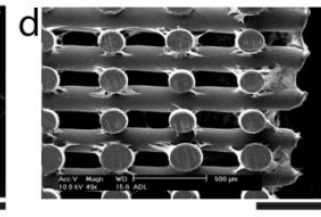
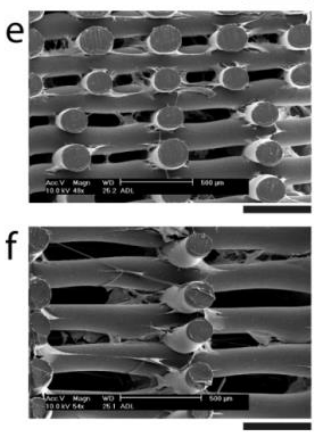

500

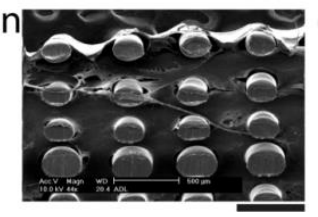

CHONDROGENIC
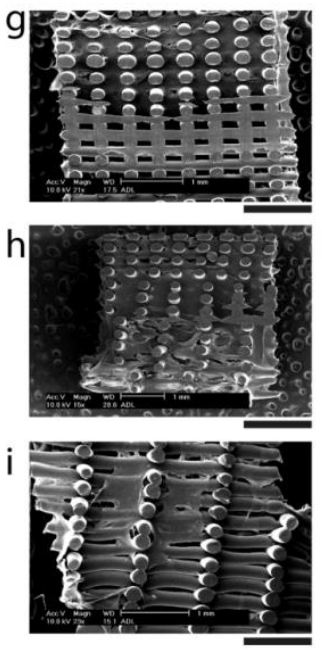

$700 / 900$

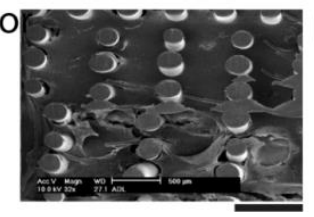

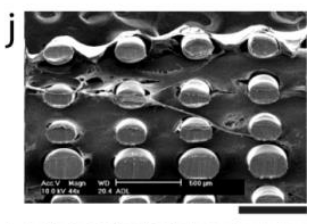
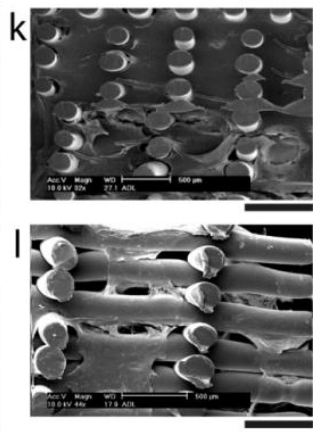

$900 / 1100$

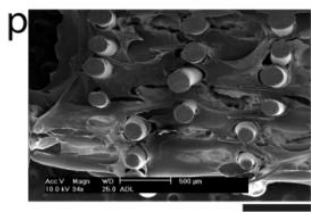

Figure 4. SEM micrograph displaying the ECM deposition of hMSCs cultured under basic (a-f) and chondrogenic medium ( $\mathrm{g}-\mathrm{l})$ after 28 days. Under chondrogenic conditions, gradient scaffolds $(h, k)$ displayed an increased amount of ECM deposited in respect of NG $500(\mathrm{~g}, \mathrm{j})$ and NG 1100 (i, I) under the same conditions. Scaffolds cultured under basic medium displayed a very low amount of ECM without major differences among the constructs. Scale bar: $1 \mathrm{~mm}(\mathrm{a}-\mathrm{c}, \mathrm{g}-\mathrm{i})$ and $500 \mu \mathrm{m}(\mathrm{d}-\mathrm{f}, \mathrm{j}-\mathrm{l})$. In the lower part of the figure $(\mathrm{m}-\mathrm{p})$ micrographs displaying the ECM organization of the entire G scaffold cultured under chondrogenic conditions and SEM pictures taken at higher magnifications of the gradient zones. Scale bar: $1 \mathrm{~mm}(\mathrm{~m})$ and $500 \mu \mathrm{m}(\mathrm{n}-\mathrm{p})$. 


\section{5 hMSC differentiation and ECM deposition in respect of the pore size zone}

\subsubsection{Gene expression per gradient zone}

As showed in figure 5, the expression of aggrecan, collagen type II and sox9 genes were analyzed in each portion of the gradient scaffolds. The early marker Sox 9 presented a 5 -fold induction in the smallest pore region, significantly higher than in the biggest pore region. The portion with the fiber spacing of $700 \mu \mathrm{m}$ presented a 2-fold induction, similar to the largest pore region. Collagen type II showed a similar trend with a 7-fold increase when the fiber spacing was set at $500 \mu \mathrm{m}$ and a 3-fold increase with a fiber spacing of $700 \mu \mathrm{m}$. Aggrecan displayed the highest differences between the portions with a 21- and a 4-fold increase in the $500 \mu \mathrm{m}$ and $700 \mu \mathrm{m}$ zones, respectively.

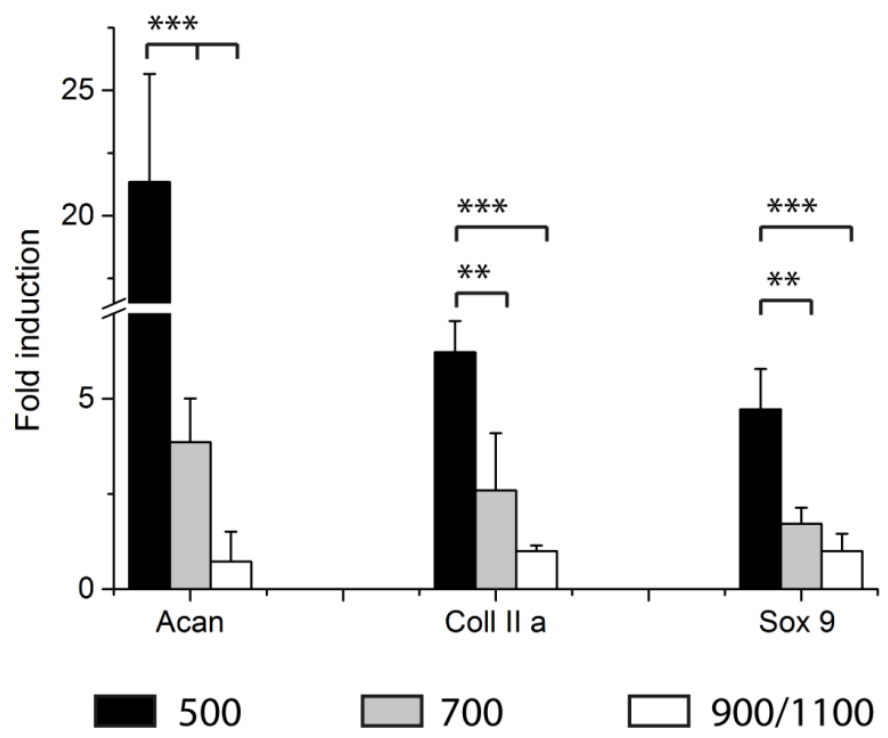

Figure 5. Differentiation of hMSCs residing in the different compartments of the gradient scaffold at genic level. The smaller pores seemed to trigger a higher expression of chondrogenic markers. All the differences between the zones were statistically significant for all the genes. Aggrecan variation was significant also between the $700 \mu \mathrm{m}$ and 900/1100 $\mu \mathrm{m}$ fiber spacing regions. ${ }^{* *}$ statistical significance $p<0.01,{ }^{* *}{ }^{*} p<0.001$. 


\subsubsection{Cellularity and ECM within the gradient}

After 28 days of culture, hMSC number decreased with increasing the pore size in chondrogenic medium (Figure 6b), while there was no difference in basic medium. GAG amount followed the same trend (Figure 6a). The portion with the smallest pore size was filled with a GAG/DNA amount of $65.06 \pm 7.85 \mu \mathrm{g}$, which decreased to $19 \pm 2.73 \mu \mathrm{g}$ in the further porosity zone and to $7.27 \pm 2.44$ in the largest pore zone (Figure 6a). A similar trend was seen under basic conditions where the 900/1100 $\mu \mathrm{m}$ portion showed a significantly lower amount of GAG/DNA with respect to the $700 \mu \mathrm{m}$ and $500 \mu \mathrm{m}$ zones, which had a similar amount. The same trend and statistical significances were maintained by normalizing the amount of GAG per zone when the structural properties were taken into consideration (Supporting Information, Figure S1). The cell number was the highest in the 500 $\mu \mathrm{m}$ part, which displayed $87564 \pm 16218$ with respect to the $700 \mu \mathrm{m}$ and $900 / 1100$ $\mu \mathrm{m}$ with $57708 \pm 7470$ and $44970 \pm 7955$, respectively. To further prove our hypothesis that protein gradient formation could be correlated to differential hypoxic conditions due to different cell nutrient availability in the different scaffold regions, we also analyzed the production of hypoxia inducible factors (HIF). Our results confirmed that in the smallest pore size region a higher HIF-1 $\alpha$ and HIF-2 $\alpha$ secretion was measured. Both HIFs production decreased with increasing pore size (Supporting Information, Figure 3). 

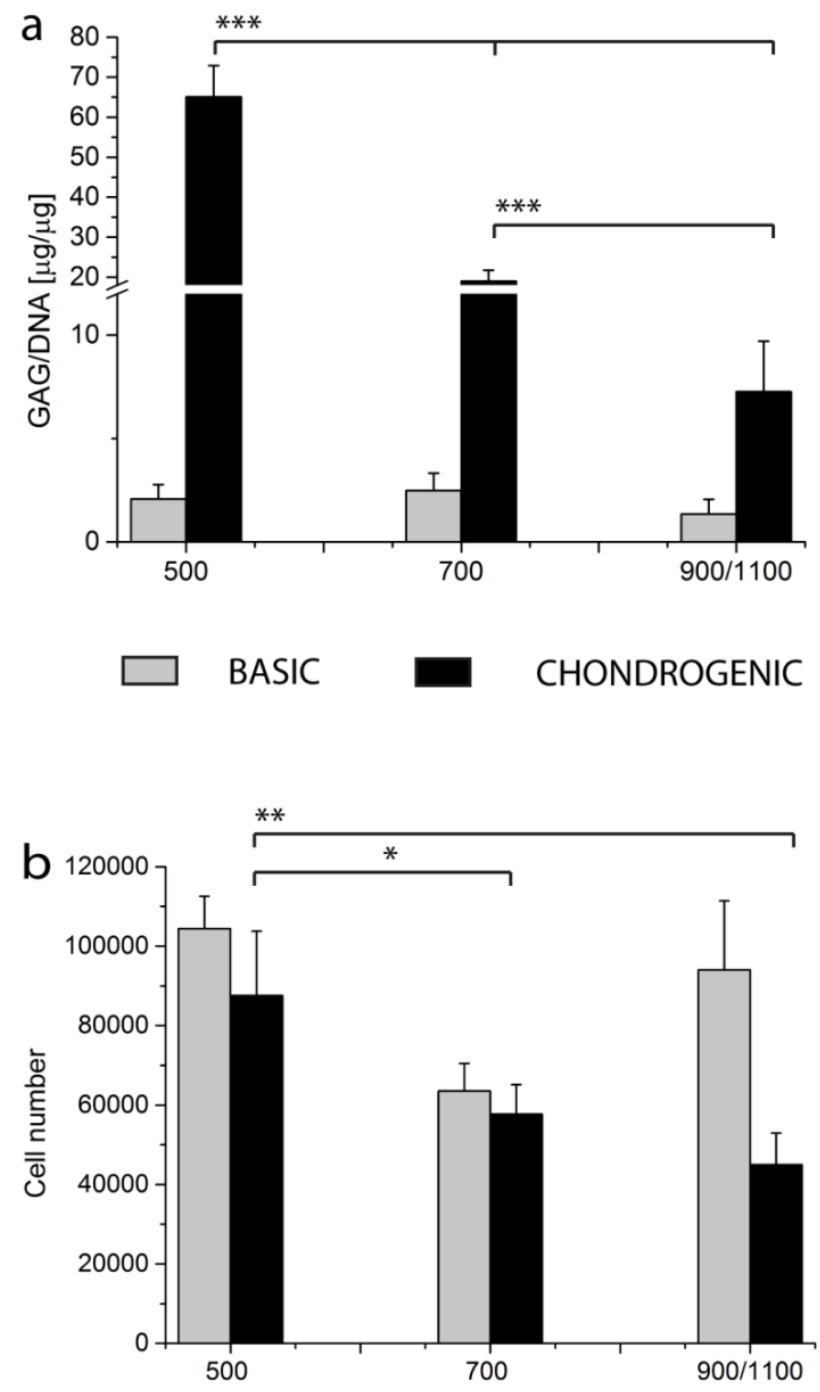

Figure 6. GAG/DNA (a) and cell number (b) in the different compartment of the gradient scaffolds. Under chondrogenic conditions both the GAG/DNA and the cell number showed a decreasing trend from the zone with smaller pores to the zone with bigger pores. Under basic conditions no trend can be seen, the cell number was higher at the bottom and top part of the scaffolds, whereas GAG/DNA was lower in the portion with bigger pores. * statistical significance $\mathrm{p}<0.05,{ }^{* *} \mathrm{P}<0.01$ and ${ }^{* * *} \mathrm{P}<0.001$. 


\section{Discussion}

In the present study, scaffolds with a discrete gradient in pore size were fabricated and showed to influence hMSC chondrogenic differentiation. The pores increased in size along the longitudinal axis by increasing the fiber spacing by $200 \mu \mathrm{m}$ every millimeter. As expected this led to an increase in porosity and pore volume. Despite some fluctuations in the fiber size, especially in the gradient scaffolds where the continuous change of the contact points between subsequent layers may have contributed to a different plastic deformation of the molten polymer during extrusion, the fabricated scaffolds displayed a controlled tunable pore network architecture. A further improvement on such fluctuations could be obtained, for example, by better controlling the heating path of the molten polymer from the cartridge where it is placed to the needle where it is extruded. Limiting the time window in which the polymer is heated would result in less thermal degradation, which would consequently result in less variations due to change of molten viscosity of the material. Other additive manufacturing technologies have been also used to create gradients in pore size, such as stereolithography [31]. In this respect, the technology that we have used is not necessarily better than other additive manufacturing technologies. The novelty of our study lies in the design of the pore size gradients specifically tuned for stem cell driven articular cartilage regeneration, while other studies using additive manufactured scaffolds focused only on improvement of cell entrapment or better cartilage regeneration with primary differentiated chondrocytes.

We expected that the gradient along the $z$ axis should have generated a sieve effect increasing cell adhesion due to an increased number of fiber connections. Sobral et al. [15] showed how a gradient structure can enhance the cell entrapment ability of a scaffold by increasing the fiber connections point where cell adhesion begins. Our findings are in contrast to this study, since no differences could be seen in cell number after 8 hours. Even though a sieve effect could be expected in our scaffolds, this did not affect the cell seeding with respect to the control scaffolds. Furthermore, to compensate as much as possible the effect of gravity on cell seeding distribution, scaffolds were flipped every day, which is known to 
improve cell homogeneous distribution in additive manufactured scaffolds [32]. The different gradient direction of the fore mentioned study and the consequent number of fibers encountered along the $z$ axis by the cells could have determined a sieve effect greater than the one showed by our scaffolds. As expected, by culturing the scaffolds in basic and chondrogenic medium a significant difference in cell number between the conditions was measured. Cell adhesion on the scaffolds is mediated by proteins such as laminin, fibronectin and vitronectin [33-35], which are commonly present in the FBS contained in the basic medium. As chondrogenic medium does not contain FBS, it is reasonable to expect a decrease in cell number with culturing time, which is also in line with the low cell density of articular cartilage tissue.

The scaffold features in terms of porosity and pore size did not affect hMSC proliferation either. It is known that structural properties such as pore shape and size affect ECM deposition [16, 36]. In particular, from the study of Woodfield et al., when cells are cultured within structures with small pores, the release of GAG is significantly enhanced with respect to structures presenting bigger pores [16]. Similarly, in our study the scaffolds presenting a homogeneous fiber spacing of 500 $\mu \mathrm{m}$ showed a significantly higher amount of GAG/DNA with respect to the NG1100 scaffolds (Figure $3 \mathrm{~b}$ ). The gradient scaffolds supported an amount of GAG/DNA which is closer to the amount presented by the scaffolds with a homogenous pore size of $326 \mu \mathrm{m} \pm 17 \mu \mathrm{m}$ and significantly higher than the amount present in the scaffolds with a homogenous pore size of $968 \mu \mathrm{m} \pm 25 \mu \mathrm{m}$.

In vivo, chondrogenesis begins from the condensation of hMSCs after the exposure to TGF- $\beta$, which leads to the expression of molecules involved in the condensation process such as tenacin $\mathrm{C}$, fibronectin and $\mathrm{N}$-chaderin $[37,38]$. Condensation leads to the expression of the transcription factor sex determining region Y-box 9 (sox-9). The cells within the aggregates proceed to chondroblasts and begin to produce aggrecan and collagen type II, IX, and XI. This development is carried on by the effect of sox-9 in combination with the influence of a growth factor cocktail, including amongst others insulin growth factor-1, fibroblast growth factor-2, and bone morphogenetic proteins (BMPs) -2, -4, -7, and -14 [37, 39, 40]. A study of Steinert et al.[41] showed a correlation between the size of cell 
aggregate, number of cells and GAG deposited. By increasing the size of the cell aggregate, the cell number and the GAG deposited also increased. In the present study, we hypothesized that the structural properties of the construct could have determined a faster filling of the smaller pores and the consequent aggregate-like state of hMSCs. This may have led to a faster and more pronounced differentiation toward the chondrogenic lineage. In the full scaffolds, the pore geometry seemed to affect only the ECM deposition and not the differentiation, since no major differences could be seen in terms of chondrogenic markers at the genic level. The ECM produced seemed to be mainly based on proteoglycans, since the synthesis of coll II mRNA was not significantly induced, whereas ACAN gene expression was enhanced in all the constructs already after 2 weeks.

A similar explanation can be proposed for the behavior of hMSCs residing in the different gradient compartments. The portion with a fiber spacing of $500 \mu \mathrm{m}$ is the one presenting the smallest pore size and the highest cell number. It is known that the initial cell density can drive the differentiation of the hMSCs [42, 43]. The highest cell density per volume unit within the $500 \mu \mathrm{m}$ part may have led the cells to assume an aggregate like state, such as the initial mesenchymal cell condensation stage of the chondrogenic differentiation. $\mathrm{N}$-cadherin mediates cellcell interaction during cell condensation. Wnt-7a positively regulates $\mathrm{N}$-cadherin expression in that stage, and it is regulated by TGF-beta. TGF-beta is added in the differentiation medium, it activates p38 MAPK pathway inhibiting the Wnt-7a/betacatenin-dependent degradation of Sox9, therefore favoring ECM deposition and chondrogenesis $[44,45]$. Bigger pores determined a delay in the activation of this process, generating a gradient in expression of chondrogenic markers opposite than the pore size increase. At the genetic level as well as at the protein level, the same trend was visible with an over expression of sox9, collagen type II and aggrecan in the small pore area compared to the $700 \mu \mathrm{m}$ and the $900 / 1100 \mu \mathrm{m}$ fiber spacing zones. In the study by $\mathrm{Oh}$ et al. the chondrogenic differentiation of hMSCs increased by increasing the pore size. In a PCL scaffolds with a gradient in pore size ranging from $90 \mu \mathrm{m}$ to $400 \mu \mathrm{m}$, the more favorable environment for chondrogenic differentiation was showed by the pore size ranging from 370-400 $\mu \mathrm{m}$ after 21 days of culture [46]. Similarly, in the present study the highest 
chondrogenic differentiation was displayed by the zone with pore size of $326 \mu \mathrm{m} \pm$ $17 \mu \mathrm{m}$, close to the optimum presented in the fore mentioned study. A further increase in pore size depicted an inverted trend with respect to Oh's study.

The GAG deposited can be determined just by the higher cell number in one compartment with respect to another. In order to decouple the effect of cell number, normalization by DNA was performed. The amount of GAG produced within the gradient zones can be expressed as total amount of GAG measured in the $500 \mu \mathrm{m}, 700 \mu \mathrm{m}$ and 900/1100 $\mu \mathrm{m}$ zones. Therefore, normalization by pore volume unit was also performed. Independently from cell density and pore volume, the gradient zone with the smallest pore always resulted with the greater amount of produced GAG. The increased cell density in the $500 \mu \mathrm{m}$ zone and the lower pore size may result in a decreased oxygen tension and nutrient availability. In literature several papers correlated the oxygen tension and chondrogenesis [47-49]. Recently, Lee et al.[50] showed the correlation between the survival pathway phosphatidylinositol 3-kinase (PI3K)/Akt/FoxO pathway, activated by hypoxia, and the increased chondrogenic differentiation of hMSCs. The low oxygen tension determines a cytosolic accumulation of hypoxia inducible factor 1 (HIF-1) and its consequent translocation in the nucleus where it acts as transcription factor. The full underlying mechanism remains still unclear [50]. Exposure to hypoxic conditions activates the PI3K/Akt pathway, involved in terminal chondrocyte differentiation, promotes chondrocytes proliferation and inhibits their hypertrophic differentiation. The enhanced chondrogenic differentiation in the small pore compartment can be based on the generation of a hypoxic environment due to the cell density and pore size. This was further supported by the analysis of HIF-1a and HIF-2 $\alpha$, which both increased with decreasing pore size in our scaffolds (Supporting Information, Figure 3).

Articular cartilage presents gradients in cell number, oxygen and nutrient availability and ECM components which follow different directions [1]. In particular, the top part of the scaffolds showed the highest cell number which is decreasing with the increasing in pore size. This would suggest the use of the small pores as superficial layer and the $900 / 1100 \mu \mathrm{m}$ portion as deep zone. In the native articular cartilage the GAG amount decreases when moving from the deep to the superficial 
layer where the chondrocytes show a lower differentiation degree. Also the nutrient availability and oxygen tension decrease from the superficial zone moving to the deep zone. According to our findings the $500 \mu \mathrm{m}$ fiber spacing zone, corresponding to a pore size of $\sim 300 \mu \mathrm{m}$, is the one showing the highest expression in chondrogenic markers, in glycosaminoglycans synthesis, as well as in HIF secretion. Despite Oh's study showed that below $300 \mu \mathrm{m}$ in pore size chondrogenesis is not improved, we could not exclude that by further decreasing the pore size below $300 \mu \mathrm{m}$ a further increase in chondrogenic differentiation could have been observed with our scaffolds as they display a completely interconnected pore network. Further studies could look into such smaller pore size, taking into account that with this technique the pore size limitation is dictated by the fiber dimension and reaches a resolution limit of approximately $100 \mu \mathrm{m}$. Further studies will be aimed at identifying more components of the ECM in the different zones. Presence and structure of collagen type II fibrils can be markers to correlate the structure of the native cartilage with the one formed within the scaffold. Additionally, an analysis on the expression of collagen $X$ may clarify if the state of hypertrophic cartilage is reached and may contribute deciding which direction to choose for the implantation of the gradient scaffold in an in vivo model. Supplementary studies are still needed to understand if the hMSCs within a porosity gradient are able to reach the state of mature cartilage and if the gradients found in the native cartilage are formed in vivo. A further application could be the use of such scaffolds for osteochondral regeneration, where the small pores are indeed used for chondrogenesis, while the larger pores are used for osteogenesis. In this respect, we have conducted additional studies on the influence of such gradient scaffolds on the osteogenic differentiation of MSCs and found out that with increasing pore size osteogenic differentiation was enhanced [51]. Nonetheless, fabricating scaffolds with structural gradients seems a promising approach to support a hierarchical chondrogenic differentiation of adult stem cells. 


\section{Conclusions}

Articular cartilage can be seen as a gradient tissue. Therefore, the use of gradient scaffolds in combination with undifferentiated cells can be foreseen as tools for its regeneration. In the present study, a scaffold presenting a gradient in porosity was successfully produced and the differentiation of hMSCs showed enhanced chondrogenic gene expression and an increased GAG deposition with decreasing the pore size, likely due to a related higher cell density and the consequent generation of hypoxic conditions. The cells growing within the gradient scaffolds seemed to support a higher ECM deposition in comparison with the NG scaffolds. The same trend was observed among the gradient zones. Structural gradients could, thus, be considered as a promising strategy for the design of scaffolds aiding to the process of adult stem cell chondrogenic differentiation.

\section{Acknowledgements}

This research was financially supported by the Technology foundation STW (STW, 11135) and by the Province of Limburg. 


\section{References}

[1] A. Di Luca, C.A. Van Blitterswijk, L. Moroni, The osteochondral interface as a gradient tissue: From development to the fabrication of gradient scaffolds for regenerative medicine. Birth Defects Res $\mathrm{C}$ Embryo Today: Reviews. 105 (2015) 34-52.

[2] A.M. Bhosale, J.B. Richardson, Articular cartilage: structure, injuries and review of management. Brit Med Bull. 87 (2008) 77-95.

[3] Y. Na, H. Oh, Y. Ahn, Y. Han, Suturing property of tough double network hydrogels for bio-repair materials. Korea-Aust Rheol J. 27 (2015) 25-31.

[4] M.A. Oberlander, C.L. Baker, B.E. Morgan. Patellofemoral arthrosis: the treatment options. Am J Orthop (Belle Mead NJ). 27 (1998) 263-70.

[5] J.R. Steadman, W.G. Rodkey, J.J. Rodrigo, Microfracture: Surgical Technique and Rehabilitation to Treat Chondral Defects. Clin Orthop Relat Res. 391 (2001) S362-S9.

[6] S. Wakitani, K. Imoto, T. Yamamoto, M. Saito, N. Murata, M. Yoneda, Human autologous culture expanded bone marrow mesenchymal cell transplantation for repair of cartilage defects in osteoarthritic knees. Osteoarthritis and Cartilage.10 (2002) 199-206.

[7] B. Johnstone, J.U. Yoo, Autologous Mesenchymal Progenitor Cells in Articular Cartilage Repair. Clin Orthop Relat Res. 367 (1999) S156-S62.

[8] E.B. Hunziker, Articular cartilage repair: are the intrinsic biological constraints undermining this process insuperable? Osteoarthritis and Cartilage. 7 (1999) 15-28.

[9] R. Gudas, R.J. Kalesinskas, V. Kimtys, E. Stankevicius, V. Toliusis, G. Bernotavicius, A. Smailys, A prospective randomized clinical study of mosaic osteochondral autologous transplantation versus microfracture for the treatment of osteochondral defects in the knee joint in young athletes. Arthroscopy. 21 (2005) 1066-75.

[10] M.T. Ghazavi, K.P. Pritzker, A.M. Davis, A.E. Gross, Fresh osteochoondral allografts for posttraumatic osteochondral defects of the knee, J Bone Joint Surg, British Volume. 79-B (1997) 1008-13.

[11] T.J. Levingstone, A. Matsiko, G.R. Dickson, F.J. O'Brien, J.P. Gleeson, A biomimetic multi-layered collagen-based scaffold for osteochondral repair. Acta Biomater. 10 (2014) 1996-2004.

[12] S.W. Suh, J.Y. Shin, J. Kim, J. Kim, C.H. Beak, D.I. Kim, H. Kim, S.S. Jeon, I.W. Choo, Effect of Different Particles on Cell Proliferation in Polymer Scaffolds Using a Solvent-Casting and Particulate Leaching Technique. ASAIO Journal. 48 (2002) 460-4.

[13] D. Sin, X. Miao, G. Liu, F. Wei, G. Chadwick, C. Yan, T. Friis, Polyurethane (PU) scaffolds prepared by solvent casting/particulate leaching (SCPL) combined with centrifugation. Mater Sci Eng C, 30 (2010) 78-85.

[14] Leatrese D. Harris B-SK, David J. Mooney. Open pore biodegradable matrices formed with gas foaming. J Biomed Mater Res. 42(3) (1998) 396-402.

[15] J.M. Sobral, S.G. Caridade, R.A. Sousa, J.F. Mano, R.L. Reis. Three-dimensional plotted scaffolds with controlled pore size gradients: Effect of scaffold geometry on mechanical performance and cell seeding efficiency. Acta Biomater. 7 (2011) 1009-18.

[16] T.B.F. Woodfield C.A. Van Blitterswijk, J. de Wijn, T.J. Sims, A.P. Hollander and J. Riesle. Polymer Scaffolds Fabricated with Pore-Size Gradients as a Model for Studying the Zonal Organization within Tissue-Engineered Cartilage Constructs. Tissue Eng. 11(9-10) (2005) 1297-311

[17] J.C. Leijten, J. Emons, C. Sticht, S. van Gool, E. Decker, A. Uitterlinden, G. Rappold, A. Hofman, F. Rivadeneira, S. Scherjon, J.M. Wit, J. van Meurs, C.A. van Blitterswijk, M. Karperien, Gremlin 1, frizzled-related protein, and Dkk-1 are key regulators of human articular cartilage homeostasis. Arthritis Rheum. 64 (2012) 3302-12.

[18] MANSOUR JM. Kinesiology: The Mechanics and Pathomechanics of Human Movement, Chapter 5, Biomechanics of Cartilage, (2003).

[19] P.L. Mente, J.L. Lewis, Elastic modulus of calcified cartilage is an order of magnitude less than that of subchondral bone. J Orthop Res. 12 (1994) 637-47.

[20] B. Rolauffs, C. Muehleman, J. Li, B. Kurz, K.E. Kuettner, E. Frank, A.J. Grodzinsky, Vulnerability of the superficial zone of immature articular cartilage to compressive injury. Arthritis Rheum. 62 (2010) 3016-27.

[21] A.J. Hayes, S. MacPherson, H. Morrison, G. Dowthwaite, C.W. Archer. The development of articular cartilage: evidence for an appositional growth mechanism. Anat Embryol. 203 (2001) 469-79.

[22] C.A. van Blitterswijk, Tissue engineering. Amsterdam; Boston: Elsevier/Academic Press; 2008.

[23] V.C. Mow, A. Ratcliffe, A.R. Poole, Cartilage and diarthrodial joints as paradigms for hierarchical materials and structures. Biomaterials.13 (1992) 67-97. 
[24] Y.X. Preeti Malladi, M. Chiou, A.J. Giaccia, M.T. Longaker. Effect of reduced oxygen tension on chondrogenesis and osteogenesis in adipose-derived mesenchymal cells. Am J Physiol Cell Physiol. 290 (2006) 1139-46.

[25] N. Zhou, N. Hu, J.Y. Liao, L.B. Lin, C. Zhao, W.K. Si, Z. Yang, S.X. Yi, T.X. Fan, W. Bao, X. Liang, X. Wei, H. Chen, C. Chen, Q. Chen, X. Lin, W. Huang. HIF-1a as a Regulator of BMP2-Induced Chondrogenic Differentiation, Osteogenic Differentiation and Endochondral Ossification in Stem Cells. Cell Physiol Biochem. 36 (2015) 44-60.

[26] R.J. Deans, A.B. Moseley. Mesenchymal stem cells: Biology and potential clinical uses. Exp Hematol. 28 (2000) 875-84.

[27] F.P. Barry,J.M. Murphy. Mesenchymal stem cells: clinical applications and biological characterization. Int J Biochem Cell Biol. 36 (2004) 568-84.

[28] P.J.Emans, E.J. Jansen, D. van lersel, T.J. Welting, T.B. Woodfield, S.K. Bulstra, J. Riesle, L.W. van Rhijn, R. Kuijer, Tissue-engineered constructs: the effect of scaffold architecture in osteochondral repair. J Tissue Eng Regen Med. 7 (2013) 751-6.

[29] M.B. Claase, D.W. Grijpma, S.C. Mendes, J.D. de Bruijn, J. Feijen, Porous PEOT/PBT scaffolds for bone tissue engineering: Preparation, characterization, and in vitro bone marrow cell culturing. $J$ Biomed Mater Res A. 64A (2003) 291-300.

[30] L. Moroni, J.R. de Wijn, C.A. van Blitterswijk. Three-dimensional fiber-deposited PEOT/PBT copolymer scaffolds for tissue engineering: influence of porosity, molecular network mesh size, and swelling in aqueous media on dynamic mechanical properties. J Biomed Mater Res A. 75 (2005) 95765.

[31] F.P. Melchels, B. Tonnarelli, A.L. Olivares, I. Martin, D. Lacroix, J. Feijen, D.J. Wendt, D.W. Grijpma, The influence of the scaffold design on the distribution of adhering cells after perfusion cell seeding. Biomaterials, 32 (2011) 2878-84.

[32] A.M. Leferink, W.J. Hendrikson, J. Rouwkema, M. Karperien, C.A. van Blitterswijk, L. Moroni, Increased cell seeding efficiency in bioplotted three-dimensional PEOT/PBT scaffolds. J Tissue Eng Regen Med. 2013.

[33] J. Wei, T. Igarashi, N. Okumori, T. Igarashi, T. Maetani, B. Liu, M. Yoshinari, Influence of surface wettability on competitive protein adsorption and initial attachment of osteoblasts. Biomedical materials. 4 (2009) 045002.

[34] E. Ruoslahti, M. Pierschbacher, New perspectives in cell adhesion: RGD and integrins. Science. 238 (1987) 491-7.

[35] R. Pytela, M.D. Pierschbacher, E. Ruoslahti, A 125/115-kDa cell surface receptor specific for vitronectin interacts with the arginine-glycine-aspartic acid adhesion sequence derived from fibronectin. Proc Natl Acad Sci U S A. 82 (1985) 5766-70.

[36] C.M. Bidan, K.P. Kommareddy, M. Rumpler, P. Kollmannsberger, P. Fratzl, J.W. Dunlop. Geometry as a factor for tissue growth: towards shape optimization of tissue engineering scaffolds. Adv Healthc Mater. 2 (2013) 186-94.

[37] H.M. Kronenberg, Developmental regulation of the growth plate. Nature. 423 (2003) 332-6.

[38] J. Chimal-Monroy, L. Diaz de Leon, Expression of N-cadherin, N-CAM, fibronectin and tenascin is stimulated by TGF-beta1, beta2, beta3 and beta5 during the formation of precartilage condensations. Int J Dev Biol. 43 (1999) 59-67.

[39] R. Pogue, K. Lyons,. BMP signaling in the cartilage growth plate. Curr Top Dev Biol. 76 (2006) 1 48.

[40] Y. Wang, Z. Cheng, H.Z. Elalieh, E. Nakamura, M.T. Nguyen, S. Mackem, T.L. Clemens, D.D. Bikle, W. Chang. IGF-1R signaling in chondrocytes modulates growth plate development by interacting with the PTHrP/lhh pathway. J Bone Miner Res. 26 (2011) 1437-46.

[41] A.F. Steinert, G.D. Palmer, C. Pilapil, U. Noth, C.H. Evans, S.C. Ghivizzani. Enhanced in vitro chondrogenesis of primary mesenchymal stem cells by combined gene transfer. Tissue Eng Part A. 15 (2009) 1127-39.

[42] M.F. Pittenger, A.M. Mackay, S.C. Beck, R.K. Jaiswal, R. Douglas, J.D. Mosca, M.A. Moorman, D.W. Simonetti, S. Craig, D.R. Marshak. Multilineage Potential of Adult Human Mesenchymal Stem Cells. Science. 284 (1999) 143-7.

[43] R. McBeath, D.M. Pirone, C.M. Nelson, K. Bhadriraju, C.S. Chen, Cell Shape, Cytoskeletal Tension, and RhoA Regulate Stem Cell Lineage Commitment. Dev Cell. 6 (2004) 483-95.

[44] R. Tuli, S. Tuli, S. Nandi, X. Huang, P.A. Manner, W.J. Hozack, K.G. Danielson, D.J. Hall, R.S. Tuan, Transforming growth factor-beta-mediated chondrogenesis of human mesenchymal progenitor cells involves $\mathrm{N}$-cadherin and mitogen-activated protein kinase and $\mathrm{Wnt}$ signaling cross-talk. J Biol Chem. 278 (2003) 41227-36. 
[45] E.J. Jin, S.Y. Lee, Y.A. Choi, J.C. Jung, O.S. Bang, S.S. Kang. BMP-2-enhanced chondrogenesis involves p38 MAPK-mediated down-regulation of Wnt-7a pathway. Molecules and cells. 22 (2006) 3539 .

[46] Se Heang Oh THK, Gun II Im, and Jin Ho Lee. Investigation of Pore Size Effect on Chondrogenic Differentiation of Adipose Stem Cells Using a Pore Size Gradient Scaffold. Biomacromolecules. 11 (2010) 1948-55.

[47] M. Hirao, N. Tamai, N. Tsumaki, H. Yoshikawa, A. Myoui, Oxygen tension regulates chondrocyte differentiation and function during endochondral ossification. J Biol Chem. 281 (2006) 31079-92.

[48] T. Bornes, N. Jomha, A. Mulet-Sierra, A. Adesida, Hypoxic culture of bone marrow-derived mesenchymal stromal stem cells differentially enhances in vitro chondrogenesis within cell-seeded collagen and hyaluronic acid porous scaffolds. Stem Cell Res Ther. 6 (2015) 84.

[49] M. Zhu, Q. Feng, L. Bian, Differential effect of hypoxia on human mesenchymal stem cell chondrogenesis and hypertrophy in hyaluronic acid hydrogels. Acta Biomater. 10 (2014) 1333-40.

[50] H.H. Lee, C.C. Chang, M.J. Shieh, J.P. Wang, Y.T. Chen, T.H. Young, T.H. Young, S.C. Hung, Hypoxia Enhances Chondrogenesis and Prevents Terminal Differentiation through PI3K/Akt/FoxO Dependent Anti-Apoptotic Effect. Sci Rep. 3 (2013) 2683.

[51] A. Di Luca, I. Lorenzo-Moldero, B. Ostrowska, A. Lepedda, C.A. van Blitterswijk, L. Moroni, Gradients in pore size enhance the osteogenic differentiation of human mesenchymal stromal cells in three-dimensional scafolds. Scientific Reports. 2016. 


\section{Supporting information}
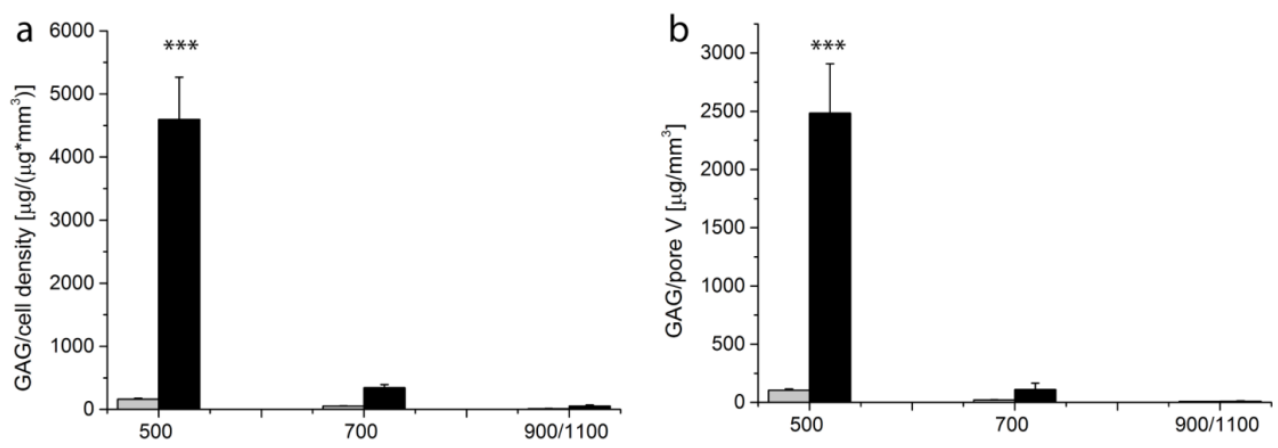

Figure S1. Graphs displaying the GAG deposited per zone (a) and the GAG deposited per zone normalized by the pore volume (b). The normalization performed did not influence the trend, the gradient portion with the smallest pore is the one displaying a greater amount of GAG in respect to the other gradient zones. ${ }^{* \star *}$ statistical significance $p<0,001$.
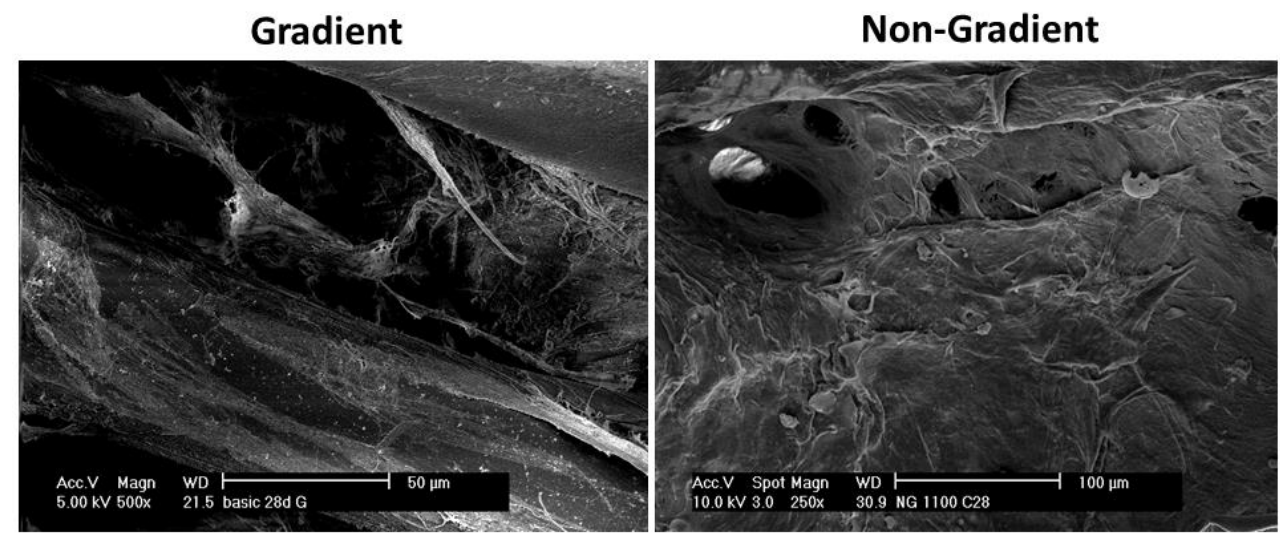

Figure S2. High magnification SEM images showing more ECM fibrillary bundles in Gradient scaffolds, while only spread cell morphology in Non-Gradient scaffolds. 

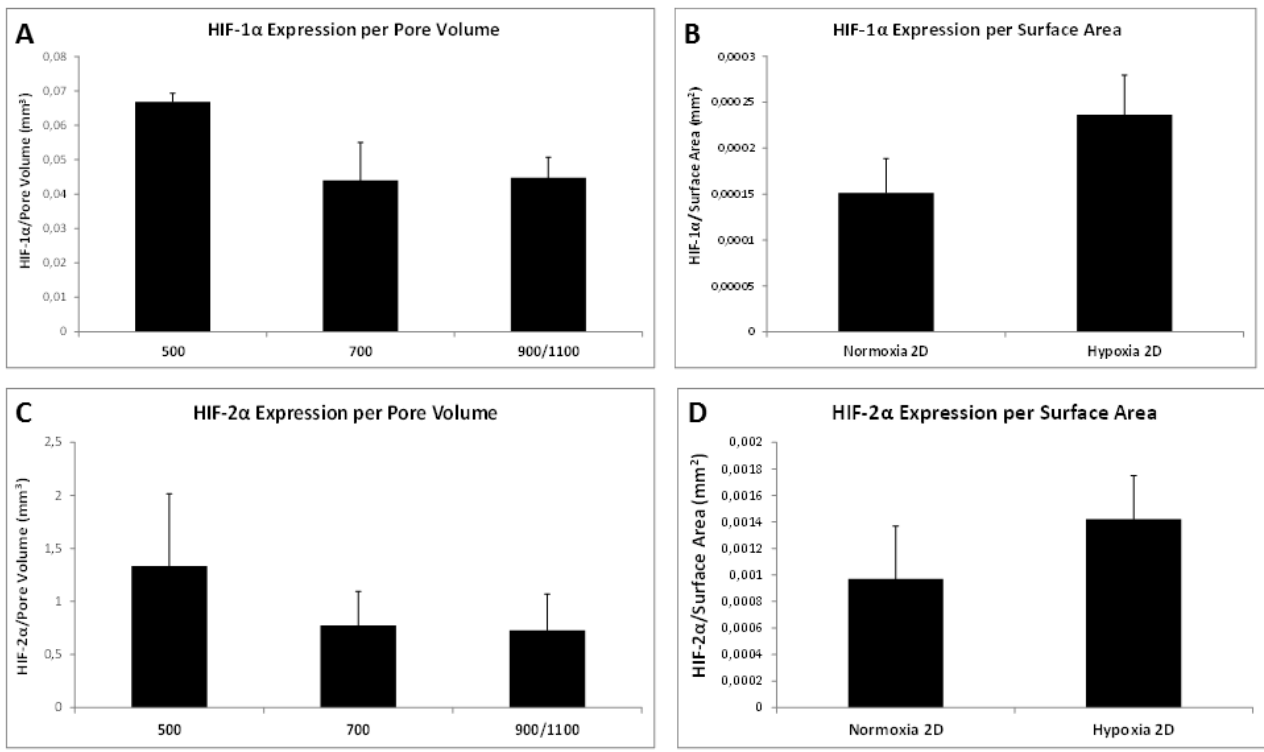

Figure S3. Partition analysis of (A) HIF-1 $\alpha$ and (C) HIF-2 $\alpha$ on different pore size regions of the gradient scaffolds show higher HIF expression for lower pore size. (C, D) Cells were also cultured in $2 \mathrm{D}$ in normoxic and hypoxic conditions as negative and positive controls, respectively. 


\section{CHAPTER 4}

\section{Toward mimicking the bone structure: design of novel hierarchical scaffolds with a tailored radial porosity gradient}

Andrea Di Luca*, Alessia Longoni*, Giuseppe Criscenti, Carlos Mota, Clemens van Blitterswijk and Lorenzo Moroni

${ }^{*}$ First shared co/authorship

Published on Biofabrication 8 (4), 045007. 2016 Oct 11. 


\section{Abstract}

Guiding bone regeneration poses still unmet challenges due to several drawbacks of current standard treatments in the clinics. A possible solution may rely on the use of three-dimensional (3D) scaffolds with optimized structural properties in combination with human mesenchymal stem cells (hMSCs). Bone presents a radial gradient structure from the outside, where the cortical bone is more compact (porosity ranging from $5 \%$ to $10 \%$ ), toward the inner part, where the cancellous bone is more porous (porosity ranging from $50 \%$ to $90 \%$ ). Here, we present a new scaffold design with a built-in gradient in porosity, which approximate the radial bone structure. The pores of the outer ring were $500 \mu \mathrm{m}$, the ones in the middle zone were $750 \mu \mathrm{m}$ and the inner part presented pores of $1000 \mu \mathrm{m}$. The porosity of each scaffold region resembled the gradient present in bone, with the outer ring having a porosity of $29.6 \% \pm 5 \%$, the middle and inner regions a porosity of $50.8 \%$ $\pm 8.1 \%$ and $77.6 \% \pm 3.2 \%$ respectively. hMSC behavior was analyzed in terms of growth, extracellular matrix (ECM) deposition and differentiation toward the osteogenic lineage. A trend was displayed by the hMSCs residing in different zones of the gradient scaffolds after 7, 14 and 28 days of culture in mineralization medium. Osteogenic differentiation was influenced by pore size and location in scaffolds displaying a radial porosity gradient. Cell differentiation was confirmed by gene expression with upregulation of Runx2 and bone sialoprotein markers. Mineralization staining further confirmed the maturation of cell differentiation, as indicated by the presence of calcium and phosphate mineral deposits. 


\section{Introduction}

Bone is the second most common transplanted tissue in the clinics. Bone is also one of the few tissues in our body with the highest capacity to regenerate after an injury. Yet, for severe critical sized defects, such regenerative capacity is impaired. Hence, bone regeneration is still a key field among regenerative medicine applications. The golden standard for bone replacement relies on autografts and allografts, the use of biological material harvested mainly from the iliac crest of the patient or from a deceased donor [1]. Among their advantages, the former possess desirable properties such as osteoinductivity and osteoconductivity [2], which are needed in order to promote bone repair. The latter present a lower percentage of graft incorporation and lower osteogenic properties. Beside their common use, these biological substitutes present several drawbacks such as donor-site morbidity (autograft), limited tissue availability and surgery complications [3]. Furthermore, allografts present the additional problem of risk of immune rejection and of pathogen transmission from donor to host [4]. In order to overcome these problems, a considerable effort has been done for developing convenient alternatives that will promote the regenerative ability of the host body [5]. Among these, the use of $3 \mathrm{D}$ scaffolds able of guiding and supporting cell activity has gained a lot of interest.

Several techniques such as gas foaming [6, 7], freeze drying [8], solvent casting and salt leaching have been used to generate scaffolds [9, 10]. Even though they are easy to implement, the resulting scaffolds lack several key properties, such as pore interconnectivity and mechanical properties, and present limitations in terms of tuning their pore network architecture. In order to overcome these limitations, in the past decade additive manufacturing (AM) emerged as alternative technique for scaffolds production. Thanks to the computer aided design and computer aided manufacturing (CAD-CAM) process, pore size and shape, fiber size and material composition can be varied during scaffold fabrication allowing, for example, the generation of gradient structures. In particular, extrusion-based AM technologies involve the use of a moving extrusion head that operates in the $X$ and $Y$ axes. The biomaterial of choice, typically a polymer, is heated above its melting temperature 
to reach a viscous state, and extruded from a nozzle in thin layers onto a stage. After the completion of each layer, the height of the extrusion head is increased and the next layer is plotted [11]. It has been already proved that this technique can be used to produce complex 3D scaffolds with defined porosity, pore size and interconnectivity [12].

Another important factor in tissue regeneration relies on the choice of a specific cell type. For bone regeneration already terminally differentiated cells such as osteoblasts can be used and would be the most logical choice. However, a further bone defect would be needed to obtain these cells. A valid alternative to osteoblasts is represented by mesenchymal stem cells (MSC), also known as skeletal stem cells or bone marrow stromal cells [13]. It has been proved that these progenitor cells can differentiate into osteogenic, chondrogenic and adipogenic lineages [14] and preserve this differentiation potential after in vitro expansion [15]. Bone marrow, periosteum, adipose tissue, umbilical cord and placenta are considered possible human MSC sources [16]. Their differentiation can be driven by several environmental cues such as material physico-chemical properties (stiffness, surface energy) [17, 18], scaffold structural properties (porosity and pore shape) [19, 20], and the presence of soluble factors (BMPs, TGF- $\beta s)$ [21-23].

Apart from the general desirable characteristics of biocompatibility, osteoinductivity and osteoconductivity, the ideal scaffold for bone tissue regeneration should possess adequate mechanical and structural properties [24]. The scaffold should have enough mechanical strength to maintain the proper architecture withstanding the hydrostatic pressure [25]. Once implanted, the construct should theoretically also match the mechanical properties of the living bone to allow an early mobilization of the injured site [26]. Regarding the scaffold architecture, the construct should have pores within the range of 200-900 $\mu \mathrm{m}$ and a fully interconnected pore network to allow cell and tissue in-growth and an efficient distribution of nutrients and oxygen [26]. However, it must be considered that an increase of the porosity coincide with a decrease of the mechanical properties [27], therefore a proper balance between these two elements is needed. Bones of the adult skeleton don't have a homogeneous structure, as they are composed by two regions that display different architectures. The outer highly dense region, known 
as cortical bone, has a porosity that ranges from $5 \%$ to $30 \%$ (mostly in the range $5 \%-10 \%$ ) whereas the inner region, called cancellous bone, has a porosity that ranges from $50 \%$ to $90 \%$. Due to the differences in porosity, cancellous bone has an elastic modulus and an ultimate compressive strength 10 times lower that the one of the cortical bone [28].

Considering these gradient structural properties related to bone porosity and pore size, an appealing new strategy to design scaffolds for bone regeneration could rely on approaching such structure to promote regional cell differentiation. We have already shown that axial gradients in pore size and architecture can improve hMSC differentiation toward the osteogenic or chondrogenic lineages [29]. Therefore, in the present study we hypothesized that it is unlikely that a uniform porous biomaterial can be suitable for cortical bone regeneration either. Inspired by cortical bone architecture, we fabricated for the first time hierarchical scaffolds with a new radial graded porosity in order to mimic the variation in bone density from the cortical bone to the cancellous bone. We studied whether such graded pore size and porosity has an influence on human MSCs behaviour.

\section{Materials and Methods}

\subsection{Scaffold design, plotting and characterization}

\subsubsection{Scaffold plotting}

Scaffolds were fabricated via an extrusion-based AM technology (Bioscaffolder, SysENG, Germany). Scaffolds were made of poly(ethylene oxide therephtalate)/poly(butylene therephtalate) (PEOT/PBT). PEOT/PBT is a family of block co-polymers characterized by an aPEOTbPBTc nomenclature, where a is the molecular weight of the starting PEG block and $b$ and $c$ are the PEOT/PBT ratio. Briefly, the syringe was filled with the polymer (300PEOT55PBT45, PolyVation, The Netherlands), heated at $190^{\circ} \mathrm{C}$, and extruded by applying a pressure of 5 bar, an auger screw rotation of $100 \mathrm{rpm}$ and a cartridge translation speed of 250-325 $\mathrm{mm} / \mathrm{min}$. The scaffold architecture was determined by a 0-90 layer configuration where fibers were deposited with a $90^{\circ}$ orientation steps between successive 
layers. The final scaffold was characterized by a cylindrical shape with a height of $3.75 \mathrm{~mm}$ and an outer diameter of $12 \mathrm{~mm}$ (the inner and middle diameter were 4 and $8 \mathrm{~mm}$, respectively). The fiber diameter and height was kept constant at 250 $\mu \mathrm{m}$ and $150 \mu \mathrm{m}$. To produce the radial porosity gradient (RPG) constructs, the fiber spacing was varied from $500 \mu \mathrm{m}$ in the outer region to $750 \mu \mathrm{m}$ in the middle region, and to $1000 \mu \mathrm{m}$ in the inner region. Three types of scaffold were used as control. Two were fabricated with a homogeneous porosity, in particular maintaining the smallest $(500 \mu \mathrm{m})$ and the biggest $(1000 \mu \mathrm{m})$ fiber spacing of the RPG scaffolds. The third one was designed with an inverted gradient of porosity (IPG), with the biggest pore volume outside and the smallest inside.

\subsubsection{Porosity Evaluation}

The porosity of the RPG and control scaffolds was evaluated following Landers et al. theoretical approach [30]:

$$
P=1-\frac{V \text { scaffold }}{V \text { cube }}=1-\frac{\pi}{4} * \frac{d_{1}^{2}}{d_{2} * d_{3}}
$$

Where, $P$ is the scaffold porosity, while $d_{1}$ is the fiber diameter, $d_{2}$ is the fiber spacing, and $d_{3}$ is the layer thickness of each scaffold.

\subsubsection{Mechanical Characterization}

The mechanical properties of RPG scaffolds and relative controls in wet and dry conditions were evaluated through uniaxial compression tests. For each configuration, 4 samples were analyzed. The length-to-diameter $(\mathrm{L} / \mathrm{d})$ aspect ratio $(1 / 3)$ provided a uniform compression stress in the region where the strain was measured. This value is the best compromise for cylindrical samples to avoid spurious bending or buckling (if $L / d>>1 / 3$ ) or a state of not uniaxial compression (if $L / d<<1 / 3$ ). In our case, $L / d \approx 0.319 \approx 1 / 3$ for the fabricated scaffolds. Samples were positioned in a standard compression block and aligned to the $500 \mathrm{~N}$ load cell of a Zwick testing machine. To evaluate the influence of an aqueous environment 
and possible perfusion effects, tests were performed under two different environmental conditions: in a nitrogen atmosphere (dry condition) and in culture medium (alpha-MEM) at $37^{\circ} \mathrm{C}$ (wet condition). In the second case, the samples were left overnight in the medium at $37^{\circ} \mathrm{C}$. All the specimens were preloaded with $0.1 \mathrm{~N}$ and preconditioned by a series of ten cycles until reaching a strain of $5 \%$ with a strain rate of $1 \mathrm{~mm} / \mathrm{min}$ to reduce the hysteresis. Subsequently, the scaffolds were compressed at $1 \mathrm{~mm} / \mathrm{min}$ until failure. From the stress-strain curve, the elastic region and the Young's Modulus $(E)$ were evaluated.

\subsection{Cell Seeding and Culture}

\subsubsection{Cell Expansion and Culture Conditions}

Pre-selected hMSCs (male, age 22) were purchased from Prockop laboratories (Temple, Texas). Cells at passage 2 were expanded at initial seeding density of about 1000 cells $/ \mathrm{cm}^{2}$ in proliferation medium at approximately $80 \%$ confluency prior the seeding on 3D scaffolds. Cells were grown at $37^{\circ} \mathrm{C}$ in a humidified atmosphere with $5 \% \mathrm{CO}_{2}$. Basic medium (BM) was used after cell seeding on the scaffolds as control for mineralization medium (MM). BM consisted of alpha minimum essential medium (MEM- $\alpha$, Gibco) supplemented with $10 \%$ fetal bovine serum (FBS, Lonza), L-glutamine (2mM, Gibco), penicillin (100 U/ml, Gibco) and streptomycin (100 $\mu \mathrm{g} / \mathrm{ml}$, Gibco), and ascorbic acid (0.2 mM, Sigma). MM, used for cell differentiation toward the osteogenic lineage, consisted of BM containing 10 $\mathrm{nM}$ dexamethasone (Sigma) and 0.01M $\beta$ -

glycerophosphate (BGP, Sigma). During cell expansion, proliferation medium, which consisted of BM supplemented with basic fibroblast growth factor $(1 \mathrm{ng} / \mathrm{ml}$, bFGF, Instruchemie) was used.

\subsubsection{Cell Seeding and Culture on FDM-scaffolds}

The scaffolds were sterilized in $70 \%$ ethanol for 45 minutes, subsequently washed twice in PBS for 5 minutes and finally incubated in BM overnight to pre-wet them and promote protein adsorption. Before seeding, scaffolds were dried and placed in a non-treated 24-well plate (NUNC). Harvested P3 hMCs were seeded on 
scaffolds with a density of 500000 cells in $100 \mu \mathrm{l}$ of BM. To obtain a homogeneous distribution in static conditions, the scaffolds were ideally divided into 4 quadrants and the initial seeding volume was split into 4 drops of $25 \mu \mathrm{l}$ (125000 cells/drop), one for each quadrant. After 4 hours of incubation in which cells were allowed to adhere, the medium was filled up to $1.5 \mathrm{ml}$. The next day, medium was changed to the experimental culture conditions (BM and $\mathrm{MM}$ ). The media were refreshed every 2-3 days and every time the scaffolds were rotated of $180^{\circ}$ in order to reduce the effect of the gravity force. Cells were grown at $37^{\circ} \mathrm{C}$ in a humidified atmosphere with $5 \% \mathrm{CO}_{2}$.

\subsection{Biochemical Analysis}

All the biochemical studies were performed after 7, 14 and 28 days of culture in MM and BM. DNA and ALP assays were performed in a "partition" manner: the three different areas of the scaffold (outer, middle and inner part) were divided using a cutter and analysed separately in order to have a better understanding of how cellular behaviour is affected by each porosity. Triplicates were used for each condition at each time point.

\subsubsection{DNA Assay}

Cell number per area was obtained from the $\mu \mathrm{g}$ of DNA, calculated with CyQuant DNA assay kit (Molecular Probes, Invitrogen) according to the manufacturer's description. Briefly, after the separation of the three areas of the scaffolds, they were further cut in order to improve the lysis efficiency. Samples were stored at -20 ${ }^{\circ} \mathrm{C}$ and freeze-thawed 5 times. Afterwards, cells within the scaffolds were lysated using the same cell lysis buffer used for the ALP activity assay. After 1 hour of incubation at room temperature, the samples were sonicated (Branson Sonifier) twice for 15 seconds. To avoid the interference caused by the binding of the dye to the RNA, $100 \mu \mathrm{l}$ of the samples were incubated for one hour at room temperature with $100 \mu \mathrm{l}$ of lysis buffer provided by the kit (Component B diluted in $180 \mathrm{mM}$ $\mathrm{NaCl}, 1 \mathrm{mM}$ EDTA in distilled water in the ratio 1:20) in which RNAse was diluted 1000 times. Quantification of the total DNA was performed using a green 
fluorescent dye provided by the kit (excitation 480nm, emission $520 \mathrm{~nm}$ ). Fluorescence was measured at $480 \mathrm{~nm}$ using a spectrophotometer LS50B (Victor 3, Perkin Elmer) and DNA concentrations were calculated from a $\lambda$ DNA standard curve. Cell density in each area of the gradient scaffolds was calculated by measuring the DNA content of each area, which was normalized by the pore volume of total amount of pores in that area of the scaffold.

\subsubsection{ALP Activity Assay}

Constructs were incubated for one hour at room temperature in a cell lysis buffer composed of $0.1 \mathrm{M} \mathrm{KH}_{2} \mathrm{PO}_{4}$ and $0.1 \%$ Triton X-100 (Acros Chemicals), at pH 7.8. ALP activity quantification was performed using a CDP-Star kit (Roche), according to the manufacturer's protocol. Briefly, $40 \mu \mathrm{l}$ of CDP-Star reagent were added to 10 $\mu \mathrm{l}$ of cell lysate. After 15 minutes of incubation at room temperature in the dark, ALP activity was measured with a spectrophotometer LS50B (Victor 3, Perkin Elmer). The obtained values were normalized to the DNA amount per area and to the single pore volume in order to take into account also the structural differences between the areas. Average conditions were represented as a relative value as all the results were compared to the ALP activity found in the inner part of the radial scaffold cultured in BM for 7 days.

\subsection{Gene Expression Analysis}

Gene expression studies were performed after 7, 14 and 28 days of culture in MM and BM. Also in this case the analyses were performed in a partition manner, dividing the three areas with different porosity. Quadruplicates were used for each condition at each time point.

\subsubsection{RNA Isolation and cDNA Synthesis}

In order to analyze the expression of osteogenic markers, samples were cut and transferred to $2 \mathrm{ml}$ eppendorf tubes. Before storing at $-80^{\circ} \mathrm{C}, 750 \mu \mathrm{l}$ of TRIzol ${ }^{\circ}$ (Invitrogen) were added. In order to isolate the RNA, $150 \mu \mathrm{l}$ of chloroform were added to all the samples and mixed by vigorously shaking the tubes. The obtained 
mixture was centrifuged at $12,000 \mathrm{~g}$ for 20 minutes at $4{ }^{\circ} \mathrm{C}$. The aqueous clear phase containing the RNA was transferred into a new eppendorf tube and mixed with an equal quantity of $70 \%$ ethanol. The product was transferred to the filter columns taken from ISOLATE II RNA mini kit (Bioline) for RNA isolation and the following steps were performed according to the manufacturer's protocol. RNA was eluted in $40 \mu \mathrm{l}$ of RNAse-free water and its concentration and purity was determined using an ND1000 spectrophotometer. For cDNA synthesis, iScript ${ }^{\mathrm{TM}}$ (Bio-Rad) was used according to manufacturer's protocol.

\subsubsection{Quantitative Polymerase Chain Reaction (qPCR)}

The obtained CDNA was used to perform $\mathrm{qPCR}$ with an $\mathrm{iQ}$ SYBR $B$ Green Supermix (Bio-Rad). Primers are listed in Table 1. PCR reactions were performed using CFX Connect ${ }^{\mathrm{TM}}$ Real-Time System (Bio-Rad) under the following conditions: cDNA was denaturated for 10 minutes at $95{ }^{\circ} \mathrm{C}$, followed by 40 cycles consisting of 15 seconds at $95^{\circ} \mathrm{C}, 15$ seconds at $60^{\circ} \mathrm{C}$ and 15 seconds at $72^{\circ} \mathrm{C}$. A melting curve was generated for each reaction in order to test primer dimer formation and nonspecific amplification. The cycle threshold (CT) values were determined using the Bio-Rad iQ5 optical system software, in which a threshold value was set for the fluorescent signal in the lower log-linear part above the baseline. The obtained CT values were normalized to the ones of the housekeeping gene (B2M) and to the $\triangle C T$, which is the subtraction of the CT value from the average of the CT of the control condition. The results were expressed as the relative mRNA expression calculated as $2-\Delta C T$, and successively normalized to the gene expression level determined in the inner part of the radial scaffold after 7 days of cell culture in BM.

\begin{tabular}{|c|c|c|}
\hline Gene & Forward primer & Reverse primer \\
\hline B2M & ACAAAGTCACATGGTTCACA & GACTTGTCTTTCAGCAAGGA \\
\hline Runx2 & TGGTTACTGTCATGGCGGGTA & TCTCAGATCGTTGAACCTTGCTA \\
\hline BSP & CCCCACCTTTTGGGAAAACCA & TCCCCGTTCTCACTTTCATAGAT \\
\hline
\end{tabular}

Table 1. List of primers used for gne expression analysis in RPG and control scaffolds. 


\subsection{Imaging}

\subsubsection{Scanning Electron Microscopy (SEM) and Energy-Dispersive X-Ray Spectroscopy (EDX) Analysis}

Cell attachment and distribution after 24 hours, 7, 14 and 28 days were observed using a Philips XL ESEM-FEG. Scaffolds were rinsed twice with PBS and fixed in $10 \%$ formalin for 30 minutes. Subsequently, the samples were cut in progressive sections and dehydrated in sequential ethanol series $(50 \%, 60 \%, 70 \%, 80 \%, 90 \%$, $96 \%$ and $100 \%$ ), 30 minutes for each concentration. For the final dehydration step, scaffolds were immersed in hexamethyldisilazane (Sigma-Aldrich)

and the solvent was left to evaporate overnight. Finally samples were gold sputtered (Cressington) prior to SEM analysis. SEM images were obtained under high vacuum with an acceleration voltage of $10 \mathrm{kV}$ and a working distance of 25 $\mathrm{mm}$. An EDX (Ametek, USA) system integrated to the SEM was used in order analyze the possible co-localization of Calcium $(\mathrm{Ca})$ and Phosphate $(\mathrm{P})$ in the extracellular matrix. The spectra were taken under high vacuum with an acceleration voltage of $10 \mathrm{kV}$ and a working distance of $10 \mathrm{~mm}$.

\subsubsection{Alizarin Red S Staining}

Calcium mineralization was qualitatively determined by alizarin red $\mathrm{S}$ staining after 28 days of culture. Samples were fixed in 10\% formalin for 30 minutes and washed twice with distilled water. Subsequently, scaffolds were cut in progressive sections in order to analyze calcium deposition also in the inner part. Each section was stained with alizarin red $S$ for 2 minutes and washed several times with distilled water. Pictures were taken using a stereomicroscope (Nikon SMZ800 with Qimaging Retiga 1300 camera).

\subsection{Statistical Analysis}

A one-way statistical analysis of variance (ANOVA) followed by a Tukey's post hoc test was used with a significant level $p$ of 0.05 to determine differences between the groups. To evaluate the differences between BM and MM, a two-tailed 
Student's t-test was performed. The confidence level was set to 0.05 . Values in this study are reported as mean and standard deviation.

\section{Results}

\subsection{Scaffold Characterization}

\subsubsection{Real Porosity Evaluation}

SEM images were used in order to measure the real values of fiber diameter (d1), fiber spacing (d2) and layer thickness (d3) (Figure 1). The values of $d 1$ and $d 3$ were theoretically kept constant in the different areas and their values were set to $250 \mu \mathrm{m}$ and $150 \mu \mathrm{m}$, respectively. The layer thickness revealed consistency between the theoretical and the real values. Moreover, it was constant in the different areas of the scaffold as $\mathrm{d} 3$ real values were $153 \mu \mathrm{m} \pm 12 \mu \mathrm{m}, 157 \mu \mathrm{m} \pm 10$ $\mu \mathrm{m}$ and $154 \mu \mathrm{m} \pm 12 \mu \mathrm{m}$ in the internal, middle and external areas, respectively. Higher differences were observed in the fiber diameter, as d1 was $202 \mu \mathrm{m} \pm 15 \mu \mathrm{m}$ in the internal part, $282 \mu \mathrm{m} \pm 29 \mu \mathrm{m}$ in the middle part and $261 \mu \mathrm{m} \pm 43 \mu \mathrm{m}$ in the external one. Fiber spacing was the only parameter that changed in order to have different pore volumes in the three areas of the RPG scaffolds. The obtained values were consistent with the theoretical ones, as d2 was $475 \mu \mathrm{m} \pm 4 \mu \mathrm{m}$ in the external part, $750 \mu \mathrm{m} \pm 26 \mu \mathrm{m}$ in the middle part and $939 \mu \mathrm{m} \pm 20 \mu \mathrm{m}$ in the internal one. The real porosities of each region are listed in Table 2. 


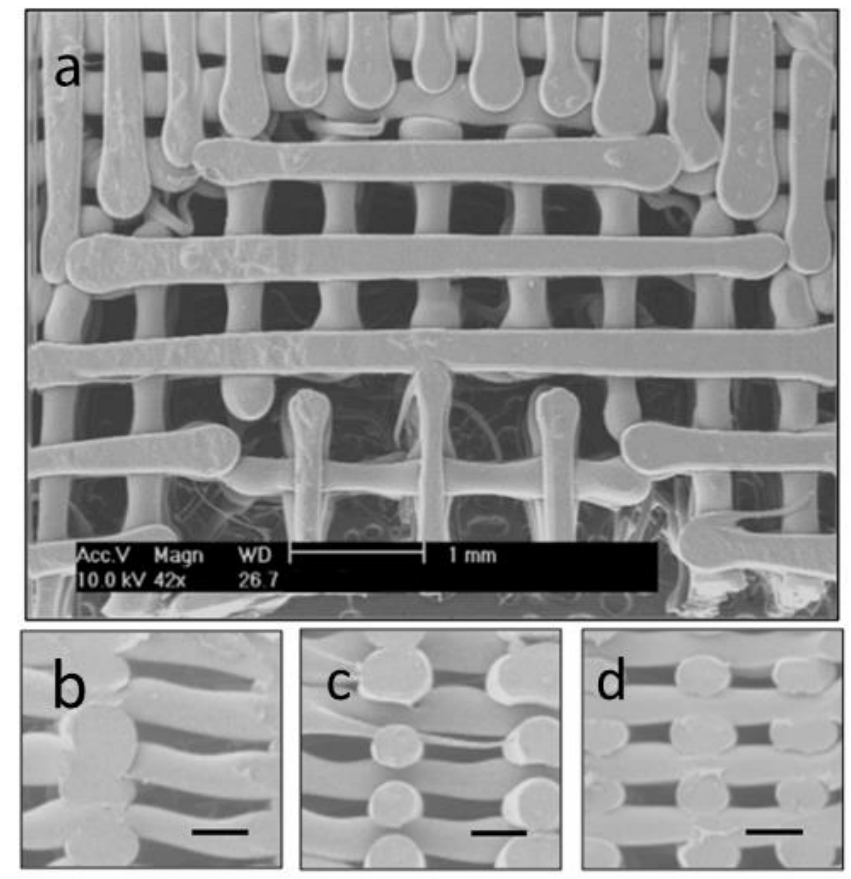

Figure 1. Figure 1. SEM micrograph showing the different areas of the RPG scaffolds. (a) represents a top view whereas (b)-(d) represent side views of the different areas of the RPG scaffolds, namely the inner (b), the middle (c), and the outer (d) ones respectively. Scale bars: (a) $1 \mathrm{~mm}$; (b)-(d) $200 \mu \mathrm{m}$.

\begin{tabular}{|c|c|c|c|}
\cline { 2 - 4 } \multicolumn{1}{c|}{} & Inner Region & Middle Region & Outer Region \\
\hline $\begin{array}{c}\text { Theoretical } \\
\text { Porosity (\%) }\end{array}$ & 68 & 56 & 35 \\
\hline $\begin{array}{c}\text { Experimental } \\
\text { Porosity (\%) }\end{array}$ & $77.6 \pm 3.2$ & $50.8 \pm 8.1$ & $29.6 \pm 5.0$ \\
\hline
\end{tabular}




\begin{tabular}{|c|c|c|c|}
\hline $\begin{array}{c}\text { Pore Volume } \\
\left(\mathrm{mm}^{3}\right)\end{array}$ & $7^{*} 10^{-2} \pm 1.8^{*} 10^{-2}$ & $2.2^{*} 10^{-2} \pm 7.1^{*} 10^{-3}$ & $5.9^{*} 10^{-3} \pm 1.7^{*} 10^{-3}$ \\
\hline $\mathbf{d}_{1}(\mu \mathrm{m})$ & $201.8 \pm 14.6$ & $282 \pm 29.1$ & $261 \pm 43$ \\
\hline $\mathbf{d}_{2}(\mu \mathrm{m})$ & $938.6 \pm 20.4$ & $750.2 \pm 26.5$ & $474.4 \pm 4.4$ \\
\hline $\mathbf{d}_{3}(\mu \mathrm{m})$ & $153.4 \pm 11.7$ & $157.2 \pm 9.8$ & $154.8 \pm 11.6$ \\
\hline
\end{tabular}

Table 2. Table displaying the experimentally evaluated structural parameters of the RPG scaffolds.

\subsubsection{Mechanical Characterization}

The mechanical properties of RPG and control scaffolds were tested in order to evaluate the influence of the different porosities. In order to take into account the structural differences between the scaffolds, the same cross-sectional area (CSA) was considered for each type of scaffold. In particular, the CSA was defined as the total surface of the scaffold considered as a bulk structure. The stress was then defined as the ratio between the applied force and the CSA. Therefore, the use of the same CSA implied a different stress value and finally a different Young's Modulus, which was related to the real structure of the scaffold. Comparing the Young's Modulus in dry conditions, the control with the smallest porosity showed a significantly higher value compared to the RPG and the IPG scaffolds (Figure 2, Tables 3 and 4). In wet conditions, no differences were observed among the samples, apart from the control with the bigger pore volume and the IPG scaffolds. Considering the RPG scaffolds, no differences were found between dry and wet conditions. When comparing the Young's Modulus of RPG scaffold with the controls with homogenous pore volume, a lower value was measured in dry 
conditions, whereas RPG scaffolds had a higher modulus similar to the homogeneous controls in wet conditions.
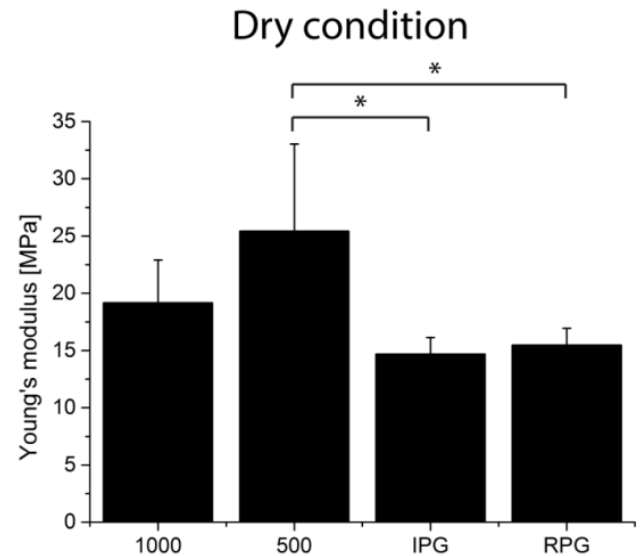

\section{Wet condition}

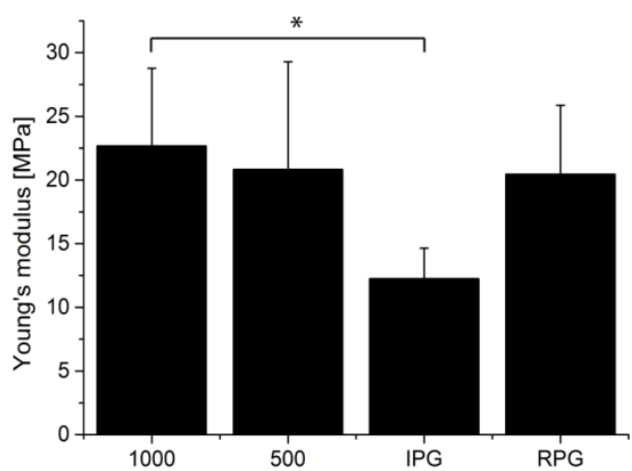

Figure 2. Young's Modulus was evaluated both in dry and wet conditions in order to simulate the culture conditions for the RPG and the controls. In dry conditions, the 500 control scaffold showed the highest Young's Modulus, whereas these differences decreased in wet conditions. ${ }^{*}$ indicates statistical significance $(P<0.05)$. RPG means radial porosity gradient; IPG means inverted radial porosity gradient; 500 and 1000 refer to scaffolds with homogenous pore size, where a fiber spacing of $500 \mu \mathrm{m}$ produced scaffolds with the smallest pore size and a fiber spacing of $1000 \mu \mathrm{m}$ produced scaffolds with the largest pore size analysed in the RPG and IPG scaffolds.

\begin{tabular}{|c|c|c|}
\hline $\begin{array}{c}\text { DRY } \\
\text { CONDITIONS }\end{array}$ & Young's modulus [MPa] & Linearity range \\
\hline 1000 & $19.17 \pm 3.73$ & $16-26$ \\
\hline 500 & $25.43 \pm 7.6$ & $19-27$ \\
\hline IPG & $14.68 \pm 1.46$ & $18-26$ \\
\hline RPG & $15.47 \pm 1.46$ & $11-18$ \\
\hline
\end{tabular}

Table 3. Young's modulus of the gradient and control scaffolds under dry conditions along with the linearity range.

\begin{tabular}{|c|c|c|}
\hline $\begin{array}{c}\text { WET } \\
\text { CONDITIONS }\end{array}$ & Young's modulus [MPa] & Linearity range \\
\hline 1000 & $22.68 \pm 6.09$ & $16-26$ \\
\hline 500 & $20.83 \pm 8.46$ & $18-30$ \\
\hline IPG & $12.26 \pm 2.39$ & $14-22$ \\
\hline RPG & $20.46 \pm 5.41$ & $18-25$ \\
\hline
\end{tabular}

Table 4. Young's modulus of the gradient and control scaffolds under wet conditions along with the linearity range. 


\subsection{Cell Seeding Efficiency and Distribution}

Cell seeding efficiency (CSE) was evaluated after 24 hours (Figure 3). CSE values ranged from $23.6 \% \pm 4.8$ to $35.8 \% \pm 7.3 \%$ and no statistically significant differences were found among the controls and the RPG scaffolds. Once it was proved that both in controls and RPG scaffolds there was approximately the same initial amount of cells, the effect of the pore size architecture on cell distribution inside the RPG scaffold was evaluated. The DNA quantification showed that there were no statistically significant differences among inner, middle and outer region. This result further confirmed that the differences in pore size and porosity did not affect cell distribution. The cells seemed to be homogeneously distributed inside the samples. In order to evaluate cell morphology and distribution, both controls and RPG scaffolds were cut in 3 progressive sections and each one was analysed using SEM. The adhered cells appeared well spread on the fibers and homogeneously distributed (Figure 3C). hMSCs seeded in the area with the lowest porosity already started to create bridges between the fibers. No cell clusters due to the cell seeding were observed. This could be due to the high surface available compared to the number of seeded cells. 
a

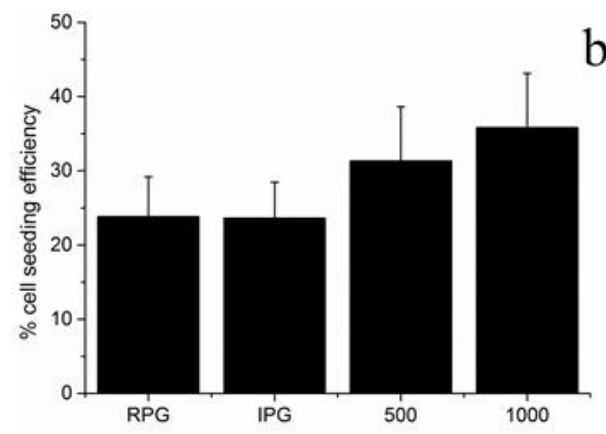

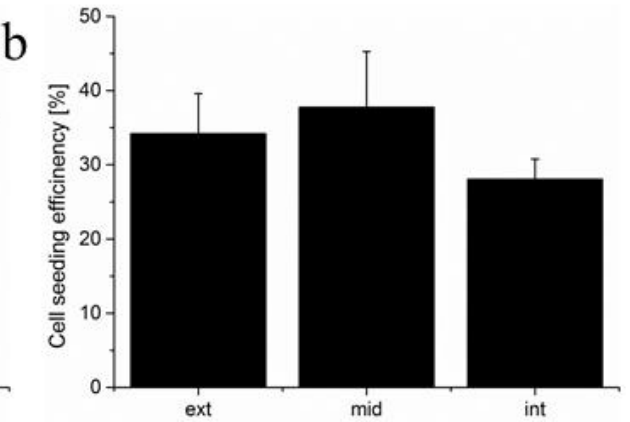

$\mathrm{C}$
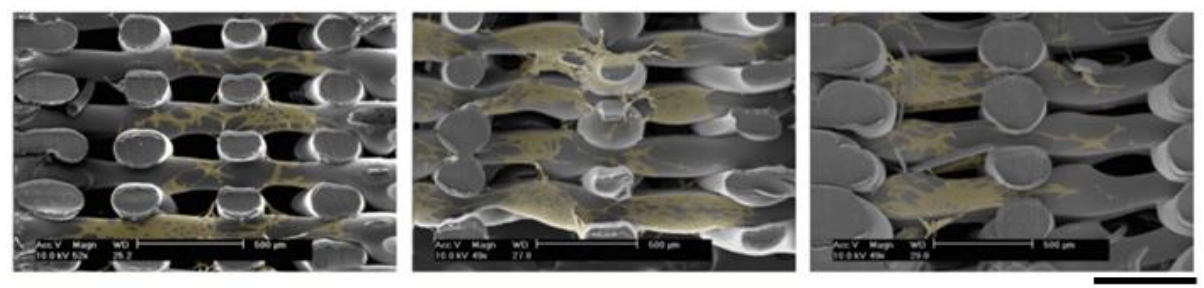

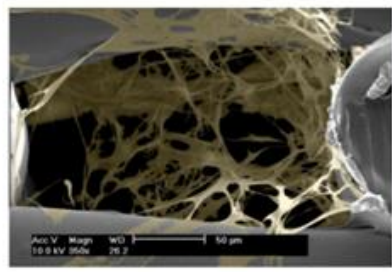

500

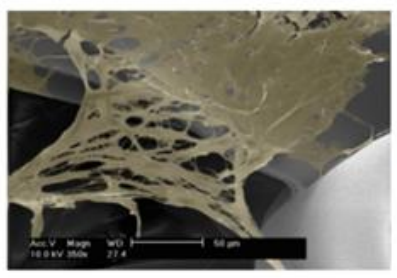

750

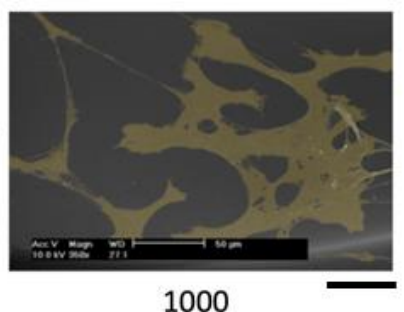

Figure3. Graphs representing cell seeding efficiency after $24 \mathrm{~h}$ in the full scaffolds (a) and in each portion of the gradient (b). SEM pictures of the 3 zones are also shown (c).No differences were observed between the RPG scaffolds and the controls, indicating that the architecture did not affect the CSE (a). Consistently, no differences were observed between the three areas with different porosities of the RPG scaffold (b). Cell spatial distribution in the three different areas after $24 \mathrm{~h}$ (c).No clusters were reported and hMSCs appeared homogeneously distributed. Scale bar: first row $500 \mu \mathrm{m}$, second row $50 \mu \mathrm{m}$. RPG means radial porosity gradient; IPG means inverted radial porosity gradient; 500 and 1000 refer to scaffolds with homogenous pore size, where a fiber spacing of $500 \mu \mathrm{m}$ produced scaffolds with the smallest pore size and a fiber spacing of $1000 \mu \mathrm{m}$ produced scaffolds with the largest pore size analyzed in the RPG and IPG scaffolds. ext, mid, and int refer to the external, middle, and internal regions in the different analyzed scaffolds.

\subsection{Effect of Porosity and Pore Size on hMSC Differentiation}

\subsubsection{Evaluation of ALP Activity}

ALP activity was evaluated after 7,14 and 28 days (Figure 4). When considering only normalization with respect to cell content, a statistically higher ALP activity was measured in MM compared to BM only after 28 days. A general trend showing 
a higher ALP/DNA ratio in the external region of the scaffolds was measured at all time points. Such trend was statistically significant in $\mathrm{MM}$ at day 14 in the 500 control scaffolds and at day 28 in 1000 control scaffolds as well as in IPG and RPG. Cell density in each area of the RPG and the control scaffolds was calculated at each time point. Higher densities were observed in the internal part of IPG scaffolds. Similarly, higher densities were found in the external region of RPG scaffolds, though at lower levels than IPG internal regions. No differences were observed between the densities of the two control scaffolds with a homogeneous porosity after 7 and 14 days. After 28 days of culture cell densities in the different areas of the control with the smallest pore size became significantly higher compared to the ones of the control with the biggest pore size (Figure 5). However, no variations were observed between the different areas within the same scaffold. In order to have a visual confirmation of the data obtained for the cell density, the samples were cut in progressive sections and analyzed with the SEM. Figure 5 shows representative pictures for each pore size and time point. The images confirmed that the pore closure process started earlier and proceeded faster in the scaffold with the smallest porosity. 

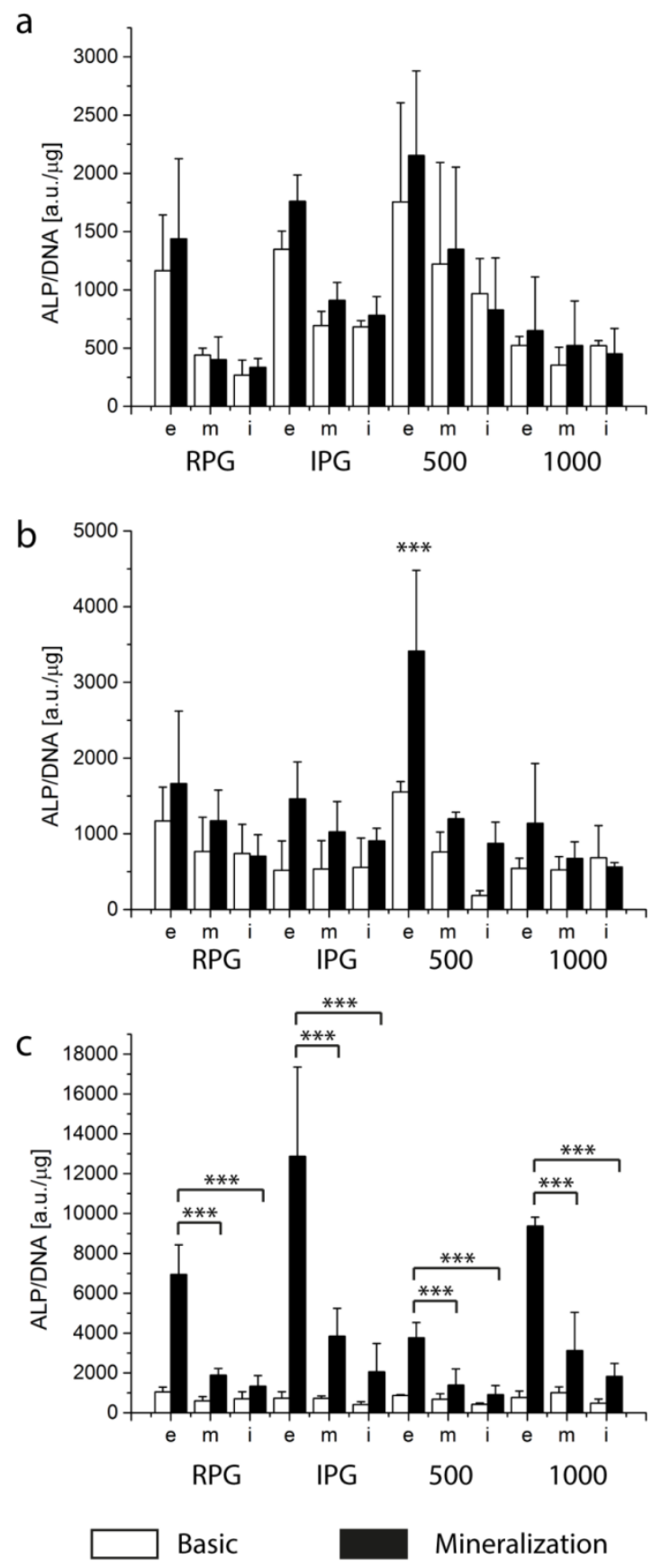

Figure 4. Plots displaying the ALP activity normalized by DNAat $7 d(a), 14 d(b)$ and $28 d(c)$, in the different zones of the non-gradient, IPG andRPG scaffolds. ${ }^{* *}$ statistical significance 
versus other zones within the same scaffold and under the same conditions, $p<0.001$. In (b), IPGe scaffolds cultured in basic medium showed statistical significance toward all the other conditions and scaffold zones with exception to IPGe scaffolds in mineralization medium. RPG means radial porosity gradient; IPG means inverted radial porosity gradient; 500 and 1000 refer to scaffolds with homogenous pore size, where a fiber spacing of $500 \mu \mathrm{m}$ produced scaffolds with the smallest pore size and a fiber spacing of $1000 \mu \mathrm{m}$ produced scaffolds with the largest pore size analyzed in the RPG and IPG scaffolds. e, $m$, and i refer to the external, middle, and internal regions in the different scaffolds analyzed.

When considering also the different pore volume in the IPG and RPG scaffolds, however, a significantly higher ALP activity was observed in the external region of the IPG scaffolds, corresponding to the largest pore size, at all time points (Figure 6). A similar trend was observed for RPG scaffolds for the internal region, again corresponding to the largest pore size, though not statistically significant compared to the other regions. 

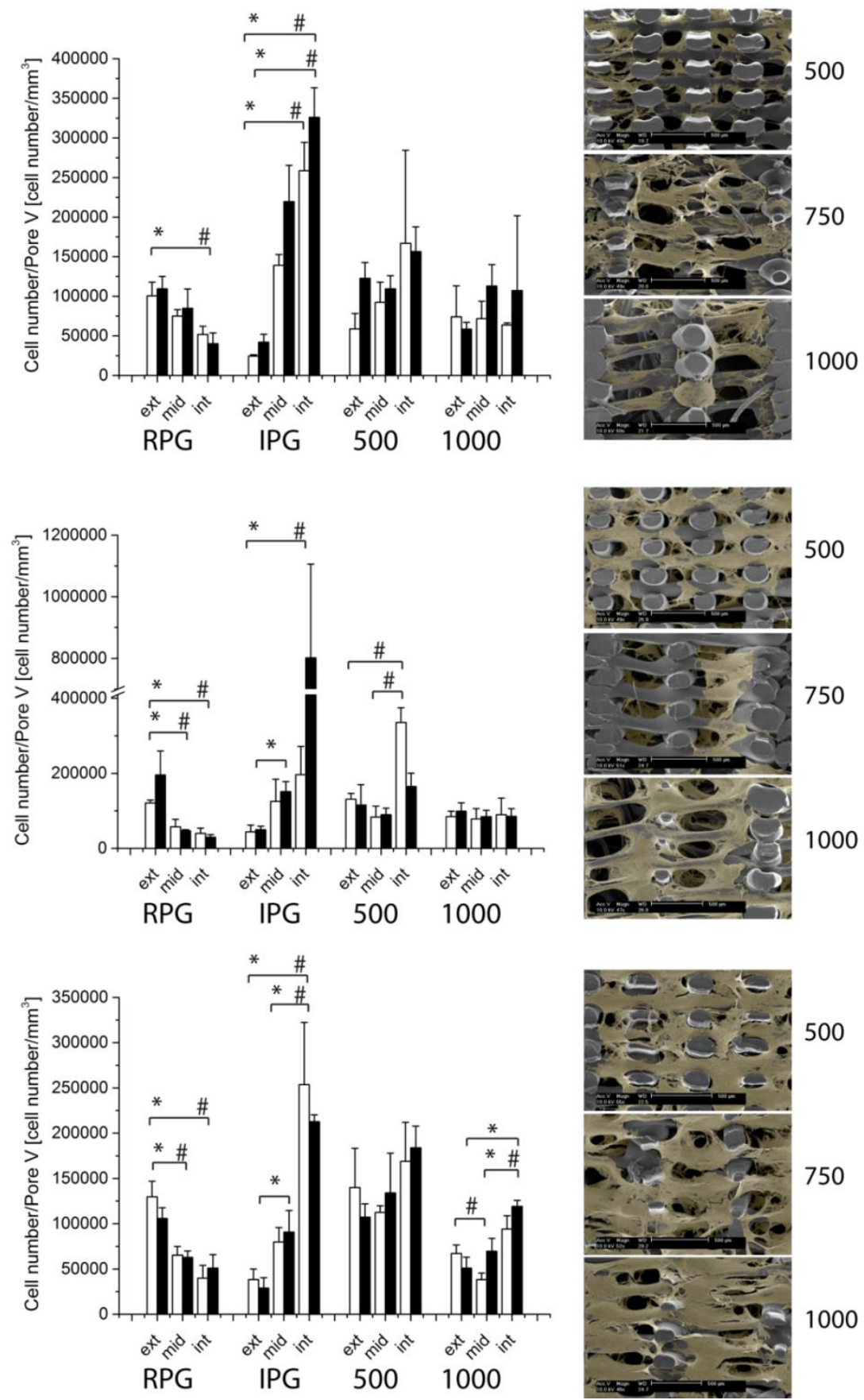

Figure 5. Cell density in the different area of control scaffolds and RPG and IPG scaffolds along time, (a)7d, (b) $14 \mathrm{~d}$ and (c) $28 \mathrm{~d}$. On the right side of each plot SEM micrograph of 
the cells residing in small pores $(500 \mu \mathrm{m})$, medium pores $(750 \mu \mathrm{m})$ and large pores $(1000$ $\mu \mathrm{m})$ at different times. IPG and RPG scaffolds displayed differences in cell density from $7 \mathrm{~d}$ whereas in scaffolds with constant pore size the differences become significant after $14 \mathrm{~d}$. * significance between mineralization conditions, \# significance between basic conditions, $\mathrm{p}<0.05$. Scale bar in SEM micrograph $500 \mu \mathrm{m}$. RPG means radial porosity gradient; IPG means inverted radial porosity gradient; 500 and 1000 refer to scaffolds with homogenous pore size, where a fiber spacing of $500 \mu \mathrm{m}$ produced scaffolds with the smallest pore size and a fiber spacing of $1000 \mu \mathrm{m}$ produced scaffolds with the largest pore size analyzed in the RPG and IPG scaffolds. ext, mid, and int refer to the external, middle, and internal regions in the different scaffolds analyzed.

\subsubsection{Gene Expression Analysis}

Gene expression of BSP and Runx 2 was evaluated after 7, 14 and 28 days of culture for RPG and IPG scaffolds. All values were normalized against the expression obtained after 7 days of culture in BM in the inner part of RPG scaffolds in order to compare the upregulation of the two genes in the different areas throughout the culture period (Figure 7). Generally, osteogenic gene expression was more upregulated in the external region of RPG and IPG scaffolds. After 14 and 28 days a higher expression of Runx2 was observed in the outer region of the RPG scaffolds characterize by smaller pore size. Consistently with Runx2 upregulation, also BSP showed an upregulation after 14 and 28 days with higher fold change in the outer region of the RPG scaffolds. In IPG scaffolds, Runx2 expression was higher in the inner part characterized by lower pore size after 14 days of culture. At day 28, however, its expression became significantly higher in the outer region characterized by larger pore size. This increase corresponded to a significant increase of BSP expression in the outer region. 

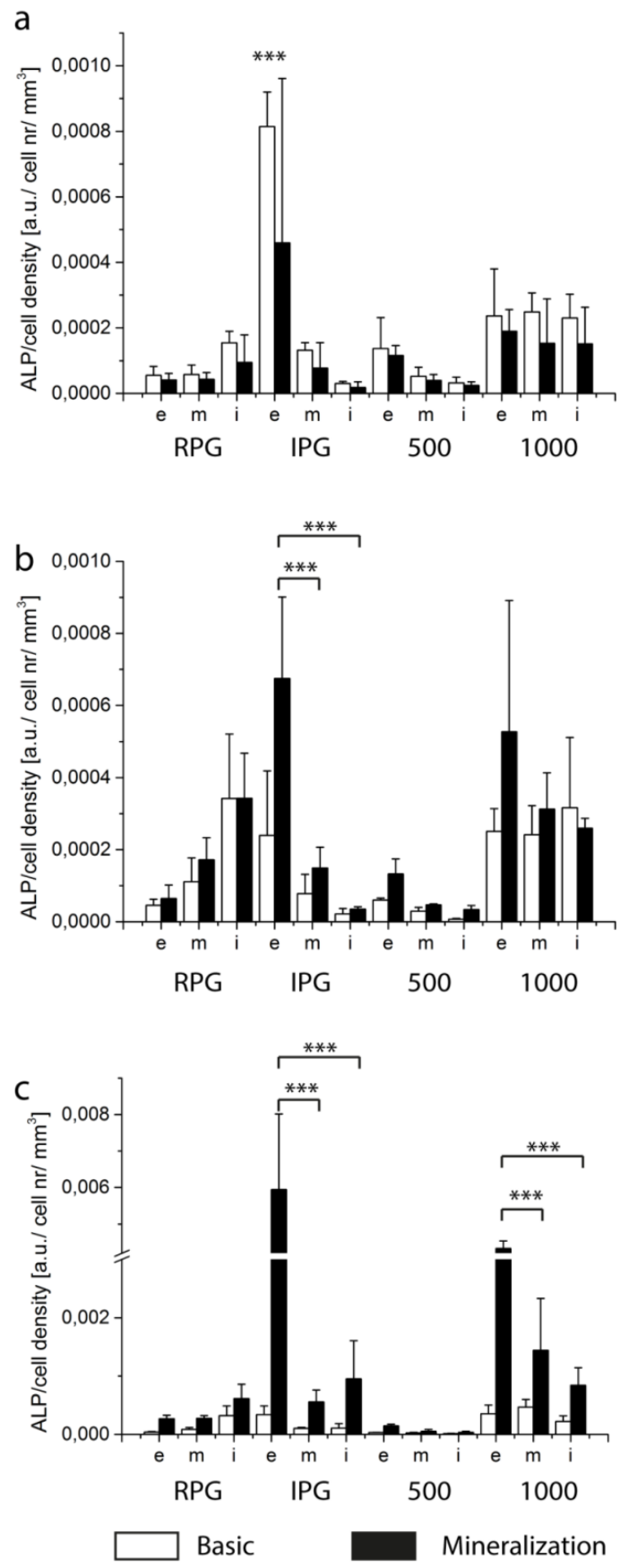

Figure 6. Graphs displaying ALP activity normalized by cell density at 7 days (a), 14 days (b) and 28 days (c), in the different zones of the non-gradient, IPG and RPG scaffolds. *** 
statistical significance versus other zones within the same scaffold and under the same conditions, $p<0.001$. In (a), IPGe scaffolds cultured in basic medium showed statistical significance toward all the other conditions and scaffold zones with exception to IPGe scaffolds in mineralization medium. RPG means radial porosity gradient; IPG means inverted radial porosity gradient; 500 and 1000 refer to scaffolds with homogenous pore size, where a fiber spacing of $500 \mu \mathrm{m}$ produced scaffolds with the smallest pore size and a fiber spacing of $1000 \mu \mathrm{m}$ produced scaffolds with the largest pore size analyzed in the RPG and IPG scaffolds. e, $\mathrm{m}$, and $\mathrm{i}$ refer to the external, middle, and internal regions in the different scaffolds analyzed.

\section{Runx2}

a

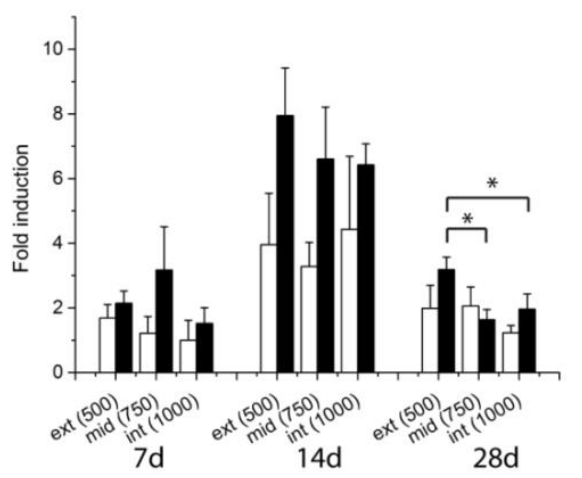

C

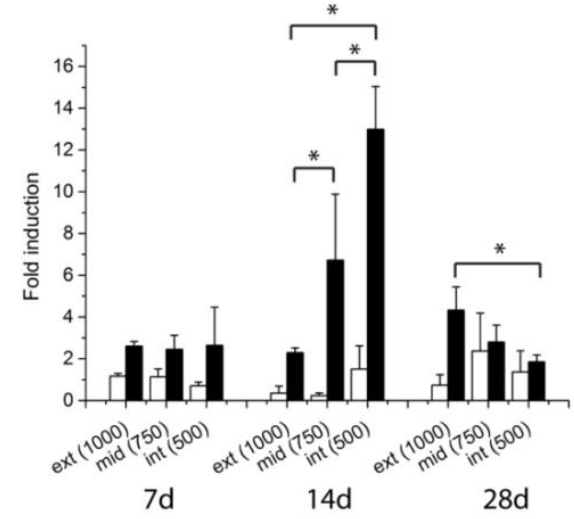

b

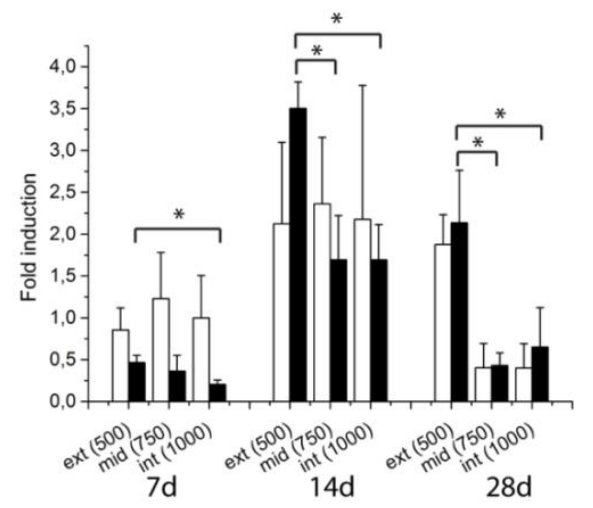

d

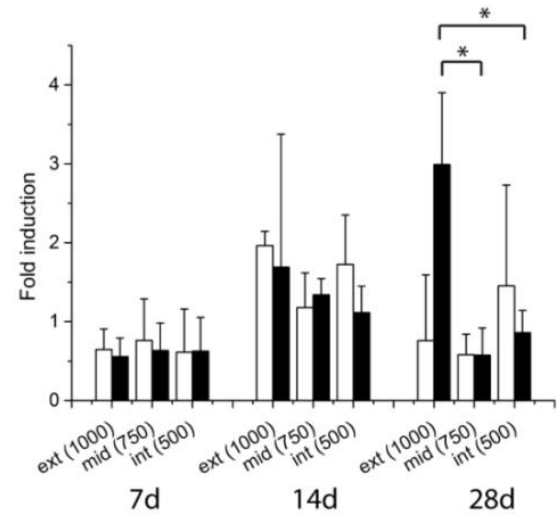

Figure 7. Runx2 and BSP expression in RPG (a, b) and IPG (c, d) scaffolds. In both types of scaffolds Runx2 showed the highest upregulation (up to 12 fold induction in the inner part of IPG scaffolds) after 14 days. BSP expression seemed to correlate with Runx2, especially in the RPG scaffolds. * indicates significantly differences $(\mathrm{P}<0.05)$ between regions. 


\subsubsection{Alizarin Red S staining and Qualitative Observation of Calcium Deposition}

An Alizarin Red S staining after 28 days of culture was employed in order to determine the presence of calcium minerals in the scaffold and analyze matrix mineralization. Despite at a first glance it was hard to observe differences between the scaffolds cultured in $\mathrm{BM}$ and $\mathrm{MM}$, at higher magnification it was possible to identify calcium deposition only on the fibers of scaffolds cultured in MM (Figure 8). No main differences were observed among the different areas of RPG scaffolds. To confirm the presence of mineralization nodules, a point analysis with the EDX was performed on the sample cultured in MM, revealing the presence of both calcium and phosphate.

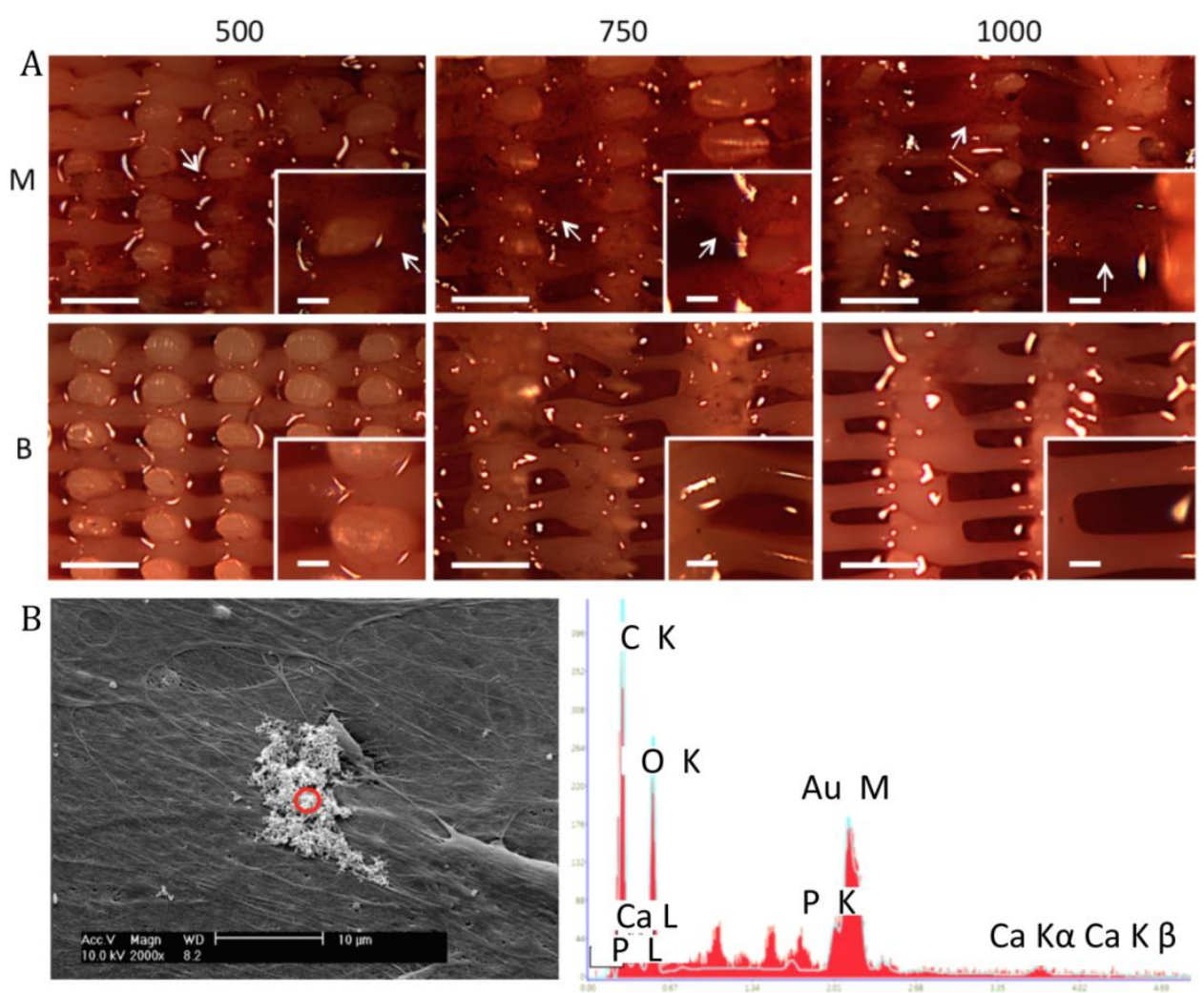

Figure 8. Calcium phosphate presence confirmed by alizarin red staining $(A)$ and SEM-EDX analysis $(B)$. White arrows $(A)$ indicate the presence of calcium on the matrix. Only few 
deposit were seen in cells after 28 days of culture in BM. Scale bars represent in the main pictures $500 \mu \mathrm{m}$ and in the insertion $100 \mu \mathrm{m}$. SEM image (B) shows a mineralization nodule. The EDX analysis confirmed the presence of both calcium and phosphate.

\section{Discussion}

A scaffold with a radial gradient structure was designed, plotted and analyzed for its capacity to guide the differentiation of hMSCs in presence and absence of osteogenic soluble factors. The experimental values of the structural parameters characterizing RPG scaffolds were similar to the designed ones and within the range of natural bone. Cortical bone has a porosity that ranges from $5 \%$ to $30 \%$ (mostly in the range of 5\%-10\%) whereas cancellous bone displays a porosity that ranges from $50 \%$ to $90 \%$ [28]. Consistently with this data, porosities of $77.6 \% \pm$ $3.2 \%, 50.8 \% \pm 8.1 \%$ and $29.6 \% \pm 5 \%$ were displayed by the outer, middle and inner region, respectively. For the inner region, the experimental porosity was higher compared to the expected one, whereas it was smaller for the middle and outer region. This difference was mainly due to the variation in fiber diameter $\left(d_{1}\right)$, which was higher than expected in the middle and external areas whereas it was smaller in the internal one. The possibility to tailor structural properties like pore size, porosity and pore distribution within the same scaffold have always appeared as an appealing tool for fundamental studies on the interactions between cells and such structural parameters as well as for more practical tissue regeneration applications. The use of gradient structures, for example, could facilitate the implant capability of withstanding different mechanical loads at specific regions minimizing stress-shielding [31]. Several materials and different techniques have been used in order to obtain a controlled structural gradient. Oh et al, for example, fabricated a gradient scaffold with a range of porosities from $80 \%$ to $94 \%$ exploiting the centrifugation of a cylindrical mould containing fibril-like polycaprolactone (PCL) followed by fibril bonding by heat treatment to study the interactions between different cell types and pore size [32]. Similarly to this work, Tampieri et al. tried to fabricate porous hydroxyapatite $(\mathrm{HA})$ bodies in order to mimic bone tissue morphology varying the characteristics of starting HA powders and the impregnation strategy of cellulosic sponges with rheologically optimized slurries 
[33]. Despite the remarkable results obtained in these studies highlighting the advantages of using gradients in scaffold design, there are only few examples in literature of their actual applications. This could be due to long and complex manufacturing procedures and to the lack of complete control over the scaffold internal architecture of the biofabrication approaches so far developed. The novelty of this study is the design and fabrication of a radial porosity graded structure exploiting AM technology. Using this technique, it was possible to overcome the above mentioned drawbacks, as the plotting procedure consisted in a less complex one-step method which allowed an excellent control over pore size, shape, orientation, and interconnection [31]. Despite this technique has already been used to produce gradient structures aimed at maximizing cell seeding efficiency [34], only another study showing the effect of pore size and porosity on chondrocyte differentiation was performed [35]. More recently, we have reported on the fabrication of axial gradient scaffolds, where pore size was changed longitudinally along the scaffolds, and showed the influence of such structural gradients on hMSCs skeletal differentiation [36, 37]. In this respect, the analysis of radial structural pore size and porosity gradients on the osteogenic differentiation capacity of hMSCs is here reported for the first time on AM scaffolds. The RPG scaffolds were characterized by a complex structure with porosity variation between regions. This implied a different cross-sectional area (CSA) in the different regions, which influenced the stress distribution on the scaffold. For this reason, the same CSA was considered for each type of analyzed scaffold in order to evaluate the influence of the structural differences. RPG scaffolds displayed a similar stiffness with respect to the controls; this is probably due to structural factors such as the orientation and relative positioning of the fibers along the scaffold, which can play a role in determining the final mechanical performance [34]. RPG and IPG scaffolds showed no statistical differences, probably because of the similar total CSA.

The initial cell seeding procedure is a critical step in a tissue engineering process. CSE and cell distribution analysis were performed in order to exclude the possibility that the further obtained results could be related to a different initial experimental condition among the RPG scaffolds and the controls. In order to minimize cell loss, 
the seeding parameters were chosen following Leferink et al. [38]. CSE values ranged from $23.6 \% \pm 4.8$ to $35.8 \% \pm 7.3 \%$, which correlated well with values in literature [39]. In contrast to recent studies, which have highlighted that in AM scaffolds the pore structure and scaffold architecture can play an important role in CSE of human osteosarcoma cells [34], no statistically significant differences were found among the controls and RPG scaffolds. Similar results were obtained by Leferink et al. comparing the CSE of hMSCs on three types of cylindrical scaffolds characterized by the same outer dimensions but different porosities. In particular, structure with porosities of $48 \%, 65 \%$ and $90 \%$ were investigated [38].

To evaluate cell differentiation, ALP activity was used as first readout. The increase in ALP activity along the entire culture period in mineralization medium represented a first indication of hMSC differentiation toward the osteogenic lineage. When taking into consideration only the cell amount in each region of the gradient scaffolds, the ALP activity seemed to be independent from the sequence of the pore size regions in the RPG And IPG scaffolds. ALP activity was significantly higher always in the external regions, being the large pore size region in the IPG scaffolds and the small pore size region in the RPG scaffolds, thus suggesting a stronger influence of nutrient (e.g. oxygen) availability compared to the scaffold's graded structural properties This difference in the enzyme activity, however, could be also correlated to different cell densities in the three regions of the RPG and the IPG scaffolds, as shown in Figure 5. Goldstein proved that in 2D the initial cell seeding density influenced both hMSC proliferation and differentiation. Dense seeding $\left(8 * 10^{4}\right.$ cells $\left./ \mathrm{cm}^{2}\right)$ reduced cells proliferation, growth and collagen synthesis whereas it appeared to enhance differentiation markers like ALP activity and mineralization [40]. Consistently with the above mentioned studies, when cell density was also taken into consideration in our studies, the ALP activity was significantly higher in the largest pore size regions (Figure 6). Cell migration could represent a possible explanation to these differences between areas: growing inside the scaffold, cells are filling the pores, thus reducing the diffusion of nutrient and oxygen. It might be that limitations in nutrient availability and the consequent hypoxic conditions led hMSC migration toward the outer part of the scaffold, creating an area of higher cell density near the edges [41]. The controls with the 
biggest pore size and the RPG scaffolds could be less affected by hMSC migration because the higher porosity of the central part of the scaffolds might allow better diffusion. Similar trends were also displayed by these scaffolds when cultured in basic medium, although the level of ALP activity expression were much lower compared to mineralization medium. Further investigations need to be performed in order to analyze oxygen and nutrient diffusion inside the different types of scaffold to confirm this hypothesis.

Despite ALP is a widely used marker in osteogenic differentiation of hMSCs, high levels of ALP activity not always correlate with the differentiation toward the osteogenic lineage. Kollmer et al. demonstrated that several markers (e.g ALP, collagen type I and osteopontin) currently used to investigate hMSC differentiation toward the osteogenic lineage are also shared with the adipogenic lineage [42]. In order to understand if the different ALP activity in the areas of the scaffolds corresponded to a heterogeneous hMSC differentiation, the gene expression of two additional markers was analyzed. Runt-related transcription factor Runx2 is an essential regulator of hMSC differentiation into the osteogenic lineage [43]. In vitro studies indicated that Runx2 triggers the expression of major bone protein genes like collagen type I (Col1a1), osteopontin, bone sialoprotein and osteocalcin during the early stage of osteoblast differentiation. Therefore, Runx2 lead to mesenchymal cells acquiring an osteoblastic phenotype, inhibiting at the same time the differentiation into adypocytes and chondrocytes. However, it also maintains osteoblastic cells in an immature stage, preventing their maturation and their transition into osteocytes [44]. Bone sialoprotein (BSP) is a phosphorylated and sulphated glycoprotein that constitutes from 8 to $12 \%$ of the total non-collagenous proteins in bone. Although BSP function has not been fully characterized yet, its expression is highly specific for mineralizing tissues [45]. After the preliminary screening phase, only IPG was used as control for the gene expression analysis, as ideally it allows monitoring at the same time the contribution of both the variables considered in this study - the pore size and the spatial distribution - on hMSC differentiation. In both RPG and IPG scaffolds, Runx2 expression decreased after 28 days of culture in mineralization medium. This might be associated with a maturation of osteoblastic phenotype, as it has been proved that Runx2 expression 
must be downregulated to mature from pre-osteoblasts to differentiated osteoblasts [46]. BSP upregulation was found consistently with Runx2 upregulation in mineralization medium, probably due to the presence OSE2 (osteoblast-specific cis-acting element, which is the binding site of Runx2)-like elements in the promoter of BSP gene [47]. The late upregulation of Runx2 and BSP in the outer part of IPG scaffolds might be explained with a delay in hMSC differentiation in the area with the higher pore volume. The trend showed by the gene analysis highlighted an increased fold induction in the area with smallest pore, independently from their localization within the scaffold. Since from the ALP and gene analysis, hMSC seemed to be proceeding toward a mature osteogenic differentiation, a late markers analysis was performed. EDX and alizarin red staining were used to analyze the mineralization of the ECM matrix. During bone mineralization, transient amorphous calcium phosphates and poorly crystalline apatite are formed. Later, these precursors undergo several crystalline phase transitions before the more stable crystalline hydroxyapatite (HA) finally forms. Anionic proteins attached to collagen are believed to be responsible for the stabilization of amorphous calcium phosphate in the early stage of bone mineralization, and the subsequent formation of nanometer-sized particles [48]. Early stage of mineralized calcium phosphate deposits could be found in RPG scaffolds in mineralization medium culture conditions (Figure 7). The identification of these firsts mineralization nodules represented a clear evidence of late hMSC maturation toward the osteoblastic phenotype [49]. Despite EDX is not ideal for making definitive statements about the stoichiometry of calcium phosphates on gold-coated samples since gold will confound the EDX phosphorus signal, the atomic spectrum together with the Alizarin Red images were clear indicators that hMSCs have deposited calcium and phosphate on the scaffold's surface [50]. SEM analysis further confirmed that the pore closure process started earlier and proceeded faster in the scaffold with the smallest porosity. Therefore, the earlier differentiation of these areas could be associated with the earlier reaching of a 3D confluence. Even though in the areas with a bigger pore size the pores were not completely closed by the bridging hMSCs, a more homogeneous tissue ingrowth was observed especially in the outer and middle part of the IPG scaffolds. In 
contrast with these observations, in the areas with the smallest pore size cells appeared to be more confluent in some part of the scaffolds but less homogeneously distributed. Similar results were obtained also by Mygind et al. [51]. These findings could provide a possible explanation for the absence of differences in cell densities between the control scaffolds and among their three areas. Therefore, it is plausible that, even though the cell number in the different area was the same, their spatial distribution affected their density locally, which could have promoted hMSC differentiation. It was proved that cadherins and cellcell gap junctions are required for full expression of the osteoblastic phenotype. For this reason it is thought that a higher cell density, which finally resulted in a higher degree of cell-cell communication, could lead to a higher rate of differentiation [52]. Although cell density seemed to supply a possible explanation for the collected data, other factors could have contributed to influence hMSC behaviour. Several studies showed that hMSC differentiation could be affected by hypoxia, as under hypoxic condition the degradation of HIF-1 $\alpha$ is prevented. This finally leads to the induction of pluripotent genes like Oct-4 and klf-4 and to the inhibition of cell terminal differentiation [53]. It is possible that the cells in the inner part of the scaffolds and in the areas with a lower pore volume are under hypoxic condition as the oxygen and the nutrient diffusion is affected by the pore closure during the culture time [54]. Therefore, a more in depth analysis of the cause of this different hMSC behavior related to the different pore size is required to assess whether cell density or local availability of oxygen could be the cause of the observed influence of pore size on hMSC osteogenic differentiation. Further studies should also aim at understanding if these results are dependent only from the structural radial gradient, or also from the chemical composition of the scaffolds. From our previous experience with axial gradients, the effect of pore size gradients on hMSC osteogenic differentiation was evident on both PEOT/PBT as well as PCL scaffolds [36]. Therefore, we could speculate that also in case of radial gradients the effect of structural properties may be predominant over the chemical composition of the scaffolds. Despite a further confirmation of these promising in vitro studies should be performed with more hMSC donors and in preclinical animal models, 
engineering structural radial gradients in 3D scaffolds could be an appealing new strategy to support adult stem cell driven bone regeneration.

\section{Conclusions}

A scaffold with an in-built radial interconnected porosity gradient was fabricated. To evaluate if hMSC behavior could be affected by the porosity and pore size of RPG scaffolds, several markers were investigated. ALP activity showed a higher activity in the areas with the larger pore size both in the RPG and IPG scaffolds when the pore architecture is taken into consideration. However, ALP activity seemed also to be dependent from the geometric location (inside vs outside) of the pore region when cellular activity is analyzed. These evidences suggested that the pore size and location could influence hMSC differentiation toward the osteoblastic phenotype. RUNX2 and BSP gene upregulation, with higher fold change in the regions with the smaller pore volume, further confirmed hMSC differentiation toward the osteogenic lineage. To have a better understanding of the maturation stage of hMSCs, a mineralization staining was performed, which highlighted the presence of calcium phosphate deposits. The presence of colocalized calcium and phosphate was confirmed through point analyses by EDX. A possible explanation for this different hMSC behavior can be related to the different cell density according to the different pore sizes in the internal, middle, and external regions of RPG scaffolds. Therefore, tailoring scaffolds' pore size might be a good strategy to affect hMSC osteogenic differentiation.

\section{Acknowledgements}

We are grateful to the Dutch Technology Foundation (STW, grant \# 11135) for financial support. This project/research was also possible thanks to the Dutch province of Limburg. 


\section{References}

1. Carter, G., Harvesting and implanting allograft bone. AORN J, 1999. 70(4): p. 660-70; quiz 672-6.

2. Ilan, D.I. and A.L. Ladd, Bone graft substitutes. Operative Techniques in Plastic and Reconstructive Surgery, 2002. 9(4): p. 151-160.

3. Giannoudis, P.V., H. Dinopoulos, and E. Tsiridis, Bone substitutes: an update. Injury, 2005. 36 Suppl 3: p. S20-7.

4. Conrad, E.U., et al., Transmission of the hepatitis- $C$ virus by tissue transplantation. J Bone Joint Surg Am, 1995. 77(2): p. 214-24.

5. Giannoudis, P.V., et al., What should be the characteristics of the ideal bone graft substitute? Injury, 2011. 42 Suppl 2: p. S1-2.

6. Leatrese D. Harris, B.-S.K., David J. Mooney, Open pore biodegradable matrices formed with gas foaming. 1998.

7. Salerno, A., S. lannace, and P.A. Netti, Graded biomimetic osteochondral scaffold prepared via CO2 foaming and micronized $\mathrm{NaCl}$ leaching. Materials Letters, 2012. 82(0): p. 137-140.

8. Levingstone, T.J., et al., A biomimetic multi-layered collagen-based scaffold for osteochondral repair. Acta Biomaterialia, 2014. 10(5): p. 1996-2004.

9. Sin, D., et al., Polyurethane (PU) scaffolds prepared by solvent casting/particulate leaching (SCPL) combined with centrifugation. Materials Science and Engineering: C, 2010. 30(1): p. 78-85.

10. Suh, S.W., et al., Effect of Different Particles on Cell Proliferation in Polymer Scaffolds Using a Solvent-Casting and Particulate Leaching Technique. ASAIO Journal, 2002. 48(5): p. 460464.

11. Hutmacher, D.W., Scaffolds in tissue engineering bone and cartilage. Biomaterials, 2000. 21(24): p. 2529-2543.

12. Iwan Zein, D.W.H., *, Kim Cheng Tan, Swee Hin Teoh, Fused deposition modeling of novel scaffold architectures for tissue engineering applications. Biomaterials, 2001.

13. Bianco, P. and P.G. Robey, Stem cells in tissue engineering. Nature, 2001. 414(6859): p. 118-121.

14. Williams, J.T., et al., Cells isolated from adult human skeletal muscle capable of differentiating into multiple mesodermal phenotypes. Am Surg, 1999. 65(1): p. 22-6.

15. Bruder, S.P., N. Jaiswal, and S.E. Haynesworth, Growth kinetics, self-renewal, and the osteogenic potential of purified human mesenchymal stem cells during extensive subcultivation and following cryopreservation. J Cell Biochem, 1997. 64(2): p. 278-94.

16. Augello, A. and C. De Bari, The regulation of differentiation in mesenchymal stem cells. Hum Gene Ther, 2010. 21(10): p. 1226-38.

17. Tse, J.R. and A.J. Engler, Stiffness Gradients Mimicking In Vivo Tissue Variation Regulate Mesenchymal Stem Cell Fate. PLoS ONE, 2011. 6(1): p. e15978.

18. Park, J.S., et al., The effect of matrix stiffness on the differentiation of mesenchymal stem cells in response to TGF-beta. Biomaterials, 2011. 32(16): p. 3921-30.

19. Kilian, K.A., et al., Geometric cues for directing the differentiation of mesenchymal stem cells. Proceedings of the National Academy of Sciences, 2010. 107(11): p. 4872-4877.

20. Kasten, P., et al., Porosity and pore size of beta-tricalcium phosphate scaffold can influence protein production and osteogenic differentiation of human mesenchymal stem cells: an in vitro and in vivo study. Acta Biomater, 2008. 4(6): p. 1904-15.

21. Chen, G., C. Deng, and Y.-P. Li, TGF- $\beta$ and BMP Signaling in Osteoblast Differentiation and Bone Formation. International Journal of Biological Sciences, 2012. 8(2): p. 272-288.

22. Re'em, T., et al., Simultaneous regeneration of articular cartilage and subchondral bone induced by spatially presented TGF-beta and BMP-4 in a bilayer affinity binding system. Acta Biomater, 2012. 8(9): p. 3283-93.

23. Reyes, R., et al., Repair of an osteochondral defect by sustained delivery of BMP-2 or TGFbeta1 from a bilayered alginate-PLGA scaffold. J Tissue Eng Regen Med, 2014. 8(7): p. 521-33.

24. Bose, S., M. Roy, and A. Bandyopadhyay, Recent advances in bone tissue engineering scaffolds. Trends Biotechnol, 2012. 30(10): p. 546-54.

25. Guarino, V., F. Causa, and L. Ambrosio, Bioactive scaffolds for bone and ligament tissue. Expert Rev Med Devices, 2007. 4(3): p. 405-18. 
26. Salgado, A.J., O.P. Coutinho, and R.L. Reis, Bone tissue engineering: state of the art and future trends. Macromol Biosci, 2004. 4(8): p. 743-65.

27. Hou, Q., D.W. Grijpma, and J. Feijen, Porous polymeric structures for tissue engineering prepared by a coagulation, compression moulding and salt leaching technique. Biomaterials, 2003. 24(11): p. 1937-1947.

28. Sikavitsas, V.I., J.S. Temenoff, and A.G. Mikos, Biomaterials and bone mechanotransduction. Biomaterials, 2001. 22(19): p. 2581-93.

29. Di Luca, A., et al., Gradients in pore size enhance the osteogenic differentiation of human mesenchymal stromal cells in three-dimensional scaffolds. Scientific Reports, 2016. 6: p. 22898.

30. Landers, R., et al., Fabrication of soft tissue engineering scaffolds by means of rapid prototyping techniques. Journal of materials science, 2002. 37: p. 3107-3116.

31. Miao, X. and D. Sun, Graded/Gradient Porous Biomaterials. Materials, 2009. 3(1): p. 26-47.

32. Oh, S.H., et al., In vitro and in vivo characteristics of PCL scaffolds with pore size gradient fabricated by a centrifugation method. Biomaterials, 2007. 28(9): p. 1664-71.

33. Tampieri, A., et al., Porosity-graded hydroxyapatite ceramics to replace natural bone. Biomaterials, 2001. 22(11): p. 1365-70.

34. Sobral, J.M., et al., Three-dimensional plotted scaffolds with controlled pore size gradients: Effect of scaffold geometry on mechanical performance and cell seeding efficiency. Acta Biomater, 2011. 7(3): p. 1009-18.

35. Woodfield, T.B., et al., Polymer scaffolds fabricated with pore-size gradients as a model for studying the zonal organization within tissue-engineered cartilage constructs. Tissue Eng, 2005. 11(9-10): p. 1297-311.

36. Di Luca, A., et al., Gradients in pore size enhance the osteogenic differentiation of human mesenchymal stromal cells in three-dimensional scaffolds. Sci Rep, 2016. 6: p. 22898.

37. Di Luca, A., et al., Influencing chondrogenic differentiation of human mesenchymal stromal cells in scaffolds displaying a structural gradient in pore size. Acta Biomater, 2016. 36: p. 2109.

38. Leferink, A.M., et al., Increased cell seeding efficiency in bioplotted three-dimensional PEOT/PBT scaffolds. Journal of Tissue Engineering and Regenerative Medicine, 2013: $p$. n/a-n/a.

39. Pfister, A., et al., Biofunctional rapid prototyping for tissue-engineering applications: $3 D$ bioplotting versus 3D printing. Journal of Polymer Science Part A: Polymer Chemistry, 2004. 42(3): p. 624-638.

40. Goldstein, A.S., Effect of seeding osteoprogenitor cells as dense clusters on cell growth and differentiation. Tissue Eng, 2001. 7(6): p. 817-27.

41. Dunn, J.C., et al., Analysis of cell growth in three-dimensional scaffolds. Tissue Eng, 2006. 12(4): p. 705-16.

42. Kollmer, M., et al., Markers Are Shared Between Adipogenic and Osteogenic Differentiated Mesenchymal Stem Cells. J Dev Biol Tissue Eng, 2013. 5(2): p. 18-25.

43. Komori, T., et al., Targeted disruption of Cbfa1 results in a complete lack of bone formation owing to maturational arrest of osteoblasts. Cell, 1997. 89(5): p. 755-64.

44. Komori, T., Regulation of osteoblast differentiation by transcription factors. J Cell Biochem, 2006. 99(5): p. 1233-9.

45. Bianco, P., et al., Expression of bone sialoprotein (BSP) in developing human tissues. Calcif Tissue Int, 1991. 49(6): p. 421-6.

46. Liu, W., et al., Overexpression of Cbfa1 in osteoblasts inhibits osteoblast maturation and causes osteopenia with multiple fractures. J Cell Biol, 2001. 155(1): p. 157-66.

47. Tu, Q., et al., Haploinsufficiency of Runx2 results in bone formation decrease and different BSP expression pattern changes in two transgenic mouse models. J Cell Physiol, 2008. 217(1): p. 40-7.

48. Song, J., V. Malathong, and C.R. Bertozzi, Mineralization of synthetic polymer scaffolds: a bottom-up approach for the development of artificial bone. J Am Chem Soc, 2005. 127(10): p. 3366-72.

49. Huang, W., et al., Signaling and transcriptional regulation in osteoblast commitment and differentiation. Front Biosci, 2007. 12: p. 3068-92.

50. Ruckh, T.T., et al., Osteogenic differentiation of bone marrow stromal cells on poly(epsiloncaprolactone) nanofiber scaffolds. Acta Biomater, 2010. 6(8): p. 2949-59. 
51. Mygind, T., et al., Mesenchymal stem cell ingrowth and differentiation on coralline hydroxyapatite scaffolds. Biomaterials, 2007. 28(6): p. 1036-47.

52. Schiller, P.C., et al., Gap-junctional communication is required for the maturation process of osteoblastic cells in culture. Bone, 2001. 28(4): p. 362-9.

53. Buravkova, L.B., et al., Mesenchymal stem cells and hypoxia: where are we? Mitochondrion, 2014. 19 Pt A: p. 105-12.

54. Karande, T.S., J.L. Ong, and C.M. Agrawal, Diffusion in musculoskeletal tissue engineering scaffolds: design issues related to porosity, permeability, architecture, and nutrient mixing. Ann Biomed Eng, 2004. 32(12): p. 1728-43. 


\section{CHAPTER 5}

\section{Tuning Cell Differentiation into a 3D Scaffold Presenting a Pore Shape Gradient for Osteochondral Regeneration}

Andrea Di Luca, Ivan Lorenzo-Moldero, Carlos Mota, Antonio Lepedda, Dietmar Auhl, Clemens van Blitterswijk, Lorenzo Moroni 


\begin{abstract}
Osteochondral regeneration remains nowadays a major problem since the outcome of current techniques is not satisfactory in terms of functional tissue formation and development. A possible solution is the combination of human mesenchymal stem cells ( $\mathrm{hMSCs}$ ) with additive manufacturing (AM) technologies to fabricate scaffolds with instructive properties. In this study, the differentiation of hMSCs within a scaffold presenting a gradient in pore shape is presented. The variation in pore shape was determined by varying the angle formed by the fibers of two consequent layers. The fiber deposition patterns were 0-90, which generated squared pores, 0-45, 0-30 and 0-15, which generated rhomboidal pores with an increasing major axis as the deposition angle decreased. Within the gradient construct, squared pores supported a better chondrogenic differentiation whereas cells residing in the rhomboidal pores displayed a better osteogenic differentiation. When cultured under osteochondral conditions the trend in both osteogenic and chondrogenic markers was maintained. Engineering the pore shape, thus creating axial gradients in structural properties, seems to be an instructive strategy to fabricate functional $3 \mathrm{D}$ scaffolds able to influence hMSC differentiation for osteochondral tissue regeneration.
\end{abstract}




\section{Introduction}

Severe injuries in the human body result in a healing process that leads to scar formation and a loss of function of the injured area. The purpose of tissue regeneration is to repair the targeted tissue restoring the function of the injured site, by combining engineer fundamentals and biological factors [1]. In the past couple of decades stem cells gathered the attention of the scientific community due to their ability to differentiate into multiple lineages [2, 3]. Human mesenchymal stromal or stem cells (hMSCs) can differentiate into osteoblasts, chondrocytes and adipocytes, among other cell types, their use does not raise the same ethical concerns of embryonic stem cells [2] and are safer in terms of potential tumor formation. Furthermore, when used in combination with biomaterials, one could think of designing three-dimensional (3D) scaffolds with instructive properties that can harness hMSC differentiation potential directly at the site of implantation.

Several environmental cues influence hMSC differentiation, such as oxygen and nutrient availability $[4,5]$, substrate characteristics such as physico-chemical and mechanical properties [6, 7], porosity and pore geometry [8-10], and the addition of soluble factors [2]. In their natural state, cells grow and solve their function in a 3D environment. For tissue regeneration purposes, many strategies have been applied to generate $3 \mathrm{D}$ structures providing a macroscopic environment that allows to lodge cells and let them produce the required proteins forming tissue extracellular matrix. Among others, salt leaching, solvent casting and gas foaming have been extensively used in the past decades [11, 12]. The major limitation of these techniques is the impossibility to tune the size, interconnectivity and geometry of the pore structure, hence impeding the perfusion of the inner part of the construct by nutrients and the migration of cells within the scaffold. A set of possible tools that enables to overcome this problem consists of additive manufacturing (AM) technologies, emerged in the regenerative medicine field in the past years due to the possibility of tuning structural parameters like the diameter, distance and orientation of the struts comprising the final 3D scaffolds. By doing this in a layerby-layer manner, the resulting scaffolds have a fully interconnected pore structures with tunable pore size and shape [10]. 
Gradients are present all over the body, leading a number of events and processes in the embryonic as well as in the adult stage of life. For example, processes such as the development of osteochondral, tendon and ligament tissues are governed by morphogens and oxygen gradients [13]. Structural gradients can be found in the body mainly at the interface between tissues. The osteochondral tissue, in particular, represents an interesting biological interface to take into consideration, both from a biological and a clinical point of view. From the subchondral bone side of the osteochondral tissue, the ECM passes from a mineralized collagen type I, to a less mineralized collagen type $X$ and a non-mineralized collagen type $I I$ in the deep zone of the articular cartilage. This variation in ECM composition continues throughout the entire articular cartilage tissue with an increase in collagen type II content and a parallel decrease of glyscosaminoglycans (GAGs) from the deep to the superficial zone. Mechanical properties and nutrient availability follow a similar trend [14]. Biologically, the mechanism at the base of stem cell driven osteochondral differentiation is not fully dissected. It is known that the presence of certain soluble factors are able to start hMSC differentiation toward the osteogenic (dexamethasone, BMP-2) $[15,16]$ or the chondrogenic lineage (insulin, TGF- $\beta 1$ and TGF- $\beta 3)[17,18]$. The regeneration of the osteochondral tissue requires, therefore, 3D scaffolds able to present the right cues in a spatially defined way. Clinically, current therapies to regenerate the osteochondral tissue are not yet completely successful. All the currently available treatments such as reparative surgery, allografts, autografts and the implantation/transplantation of autologous chondrocytes inflict further tissue destruction in other healthy tissue sites before any therapeutic effect can be achieved. Furthermore, some of these clinical treatments are additionally limited to the availability of donor supply and lead to the formation of fibrocartilage, thus not restoring the proper function of hyaline cartilage $[19,20]$.

Among all the cues influencing cell behaviour, scaffold's pore geometry and shape is known to play an important role. Pore geometry is known to have an effect on tissue growth within a pore in vitro and in vivo [21, 22]. The study of Berner et al. showed increased cranial bone healing and mechanical properties when the fiber's laydown pattern was $0 \% 90^{\circ}$ with respect to $0 \% 60 \% / 120^{\circ}$ [22]. Bidan et al. showed 
that when the MC-3T3 pre-osteoblasts were seeded into scaffolds displaying squared and cross-shaped pores, a different profile of ECM deposition could be observed, with an initial ECM deposition increased in cross shaped pores [21]. Hence, the design of scaffolds with structural gradients such as pore shape may stimulate the differentiation of hMSCs and lead to improved tissue regeneration strategies.

The concept of gradient has been applied already in different studies in 2D and 3D [23-30]. The work of Woodfield and Sobral displayed an improved cell seeding efficiency on AM scaffolds presenting a pore-size gradient for already differentiated cells such as bovine chondrocytes and human osteosarcoma cells (SaOs-2), respectively [10,31]. Yet, no study focused on the effect of scaffolds's structural gradients on the differentiation of hMSCs. Here, we present 3D scaffolds with a gradient in pore shape and we analyse the influence of such structural gradients on seeded hMSC activity for osteochondral tissue regeneration. We further dissect the structural gradient in order to highlight the differences in behaviour among the compartment of the construct based on changes in pore shape.

\section{Results}

\subsection{Construct Analysis}

A gradient structure was plotted by varying the fiber deposition pattern along the $Z$ axis. The resulting fiber diameter and fiber height were not affected by the angle deposition, being $150 \mu \mathrm{m} \pm 20 \mu \mathrm{m}$ and $140 \mu \mathrm{m} \pm 20 \mu \mathrm{m}$, respectively. On the other hand the fiber spacing varied from one gradient region to another. Squared pores displayed a fiber spacing of $510 \mu \mathrm{m} \pm 30 \mu \mathrm{m}, 0-45$ deposition pattern showed a fiber spacing of $490 \mu \mathrm{m} \pm 40 \mu \mathrm{m}, 0-30$ and $0-15$ had a fiber spacing of $530 \mu \mathrm{m} \pm 20$ $\mu \mathrm{m}$ and $480 \mu \mathrm{m} \pm 20 \mu \mathrm{m}$ respectively. As showed in Table 1, the change of the fiber deposition pattern resulted in a variation of the pore shape and size whereas the porosity was not affected. The 0-90 configuration determined squared pores and a porosity of $74 \% \pm 3 \%$, which became rhomboidal with a porosity of $72 \% \pm$ $5 \%$ by varying the deposition pattern to $0-45$. The further decrease in angle deposition resulted in stretching the rhomboidal pores with an increase of the major 
diagonal and a parallel decrease of the minor diagonal. The fiber deposition pattern 0-30 and $0-15$ showed a porosity of $75 \% \pm 7 \%$ and $73 \% \pm 6 \%$, respectively. Beside the variation in pore shape all the pore features (perimeter, area and volume) changed along the gradient direction with a minor difference between 0-90 and 045 angle depositions and a marked one among 0-45, 0-30, and 0-15 angle depositions. Scaffolds with constant pore geometry (0-90 and 0-15) were plotted and used as controls. The scaffolds were cylindrical in shape with a diameter of 6 $\mathrm{mm}$ and a height of $3.6 \mathrm{~mm}$. The porosity of the gradient (G) scaffolds was $74 \% \pm$ $5 \%$, while 0-90 non-gradient (NG90) and 0-15 non-gradient (NG15) scaffolds showed a porosity of $74 \% \pm 3 \%$ and $73 \% \pm 6 \%$ respectively. Mechanical properties analysis (Figure 1) indicated that $G$ scaffolds have a stiffer response to compression for a force range of $1 \mathrm{~N}$ compared to NG90 and NG15 scaffolds, respectively. The recovery of gradient scaffolds appeared less prone to hysteresis effects compared to non-gradient scaffolds. However, for a force range between $5 \mathrm{~N}$ and $10 \mathrm{~N}$, the loading and unloading response became similar (Figure 1b). The total deformations upon compression were in the range of $2 \%$ to $6 \%$ of height reduction, thus well within the elastic region. 


\begin{tabular}{|l|c|c|c|c|}
\hline $\begin{array}{c}\text { Fiber } \\
\text { deposition } \\
\text { pattern }\end{array}$ & $0-15$ & $0-30$ & $0-45$ & $0-90$ \\
\hline & $\begin{array}{c}\text { Rhomboid } \\
1=9 \pm 0.13 \mathrm{~mm}\end{array}$ & $\begin{array}{c}\text { Rhomboid } \\
1=1.04 \pm 0.06 \\
\mathrm{~mm}\end{array}$ & $\begin{array}{c}\text { Rhomboid } \\
1=0.75 \pm 0.04 \\
\mathrm{~mm}\end{array}$ & $\begin{array}{c}\text { Square } \\
1=0.56 \pm 0.03 \\
\mathrm{~mm}\end{array}$ \\
\hline & $15^{\circ}$ & & & \\
\hline
\end{tabular}

Table 1. The variation in pore size determined an increase in pore perimeter, area and volume from squares to rhomboids without significantly affecting the porosity. 

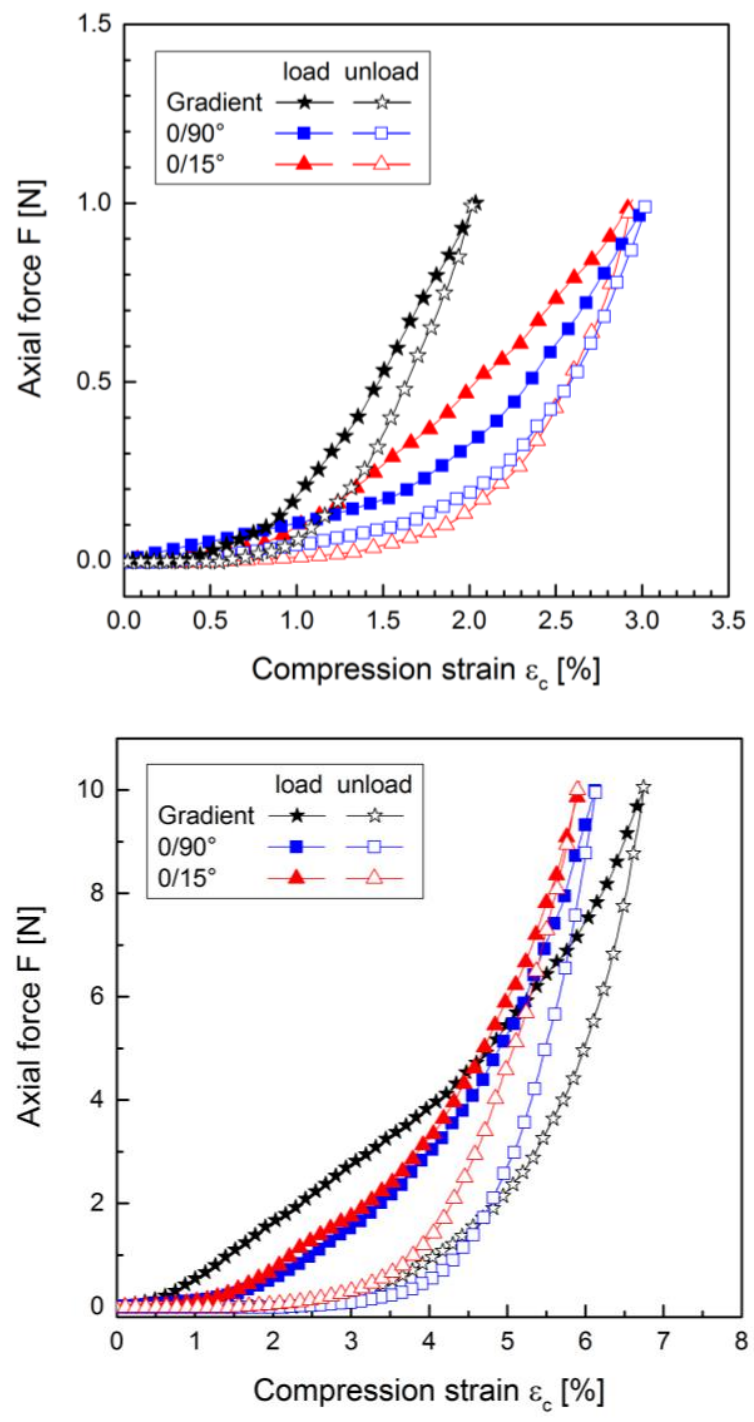

Figure 1. Mechanical response of gradient and non-gradient $\left(0 / 15^{\circ}\right.$ and $\left.0 / 90^{\circ}\right)$ scaffolds to axial compression load and unload with $1 \mu \mathrm{m} / \mathrm{s}$ in the axial $z$ direction at $37^{\circ} \mathrm{C}$ up to (left) $1 \mathrm{~N}$ and (right) $10 \mathrm{~N}$.

\subsection{Cell seeding, growth, and ECM deposition}

Cell seeding efficiency was not significantly affected by the geometry of the construct (Figure S1). The NG15 scaffolds showed a lower cell adhesion, about $60 \%$, in the first 8 hours. The gradient and the NG90 scaffolds showed a cell 
seeding efficiency of about $75-80 \%$. The direction of the gradient didn't affect cell attachment after 8 hours (Figure S1). For the rest of the study, the chosen gradient direction during the seeding was maintained with squared pores at the bottom and rhomboidal on top of the scaffolds.

Cell number increased in the first week under proliferation conditions and kept increasing also in the first week of differentiation. During the following 3 weeks of differentiation, the cell number decreased in the first week and remained stable for the last 2 weeks of culture (Figure 2). Under osteogenic and chondrogenic conditions the cell number maintained the same trend (Figure S2).

Despite the absence of differences in cell number, after 35 days of culture ( 7 days in proliferation medium followed by 28 days in basic, osteogenic, or chondrogenic medium) the gradient constructs showed an increased ECM production compared to the controls (Figure 3a), with a more rounded cell morphology on the 0-90 areas compared to the 0-15 regions (Figure S3). In NG15 and NG90 scaffolds, cells mainly wrapped the fibers (Figure $3 e, f$ ) and in some cases bridged some fibers in NG90 (Figures $3 e, h$ ). In the gradient construct the ECM was not only bridging the fibers, but filling the pores as well (Figures $3 a, d, g$ ).

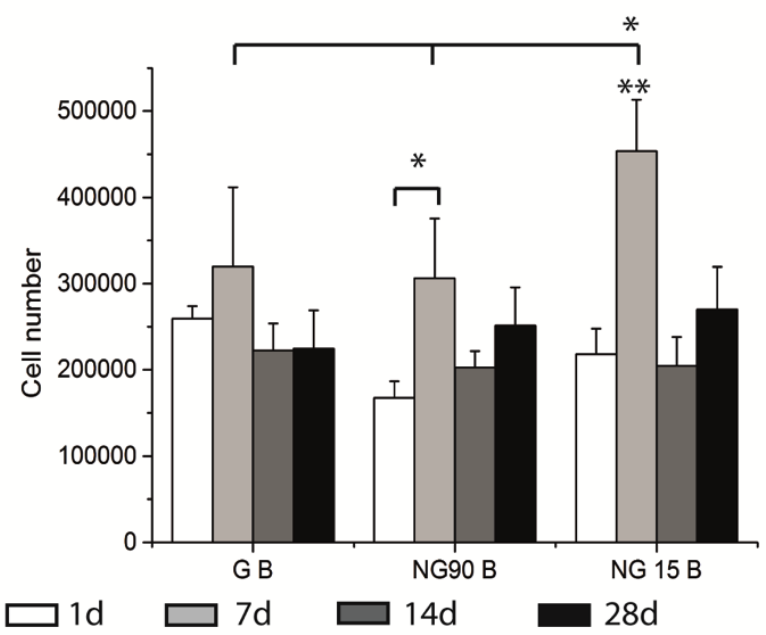

Figure 2. Cell number over time in the constructs cultured in basic medium. The same trend was shown by all the constructs over time. No major differences could be seen among the scaffolds at the same time point, with the exception of NG15B at 7 days that was 
significantly higher in respect to the other conditions and time points. * Statistical significance $p<0,05 ;{ }^{* *} p<0,01$.
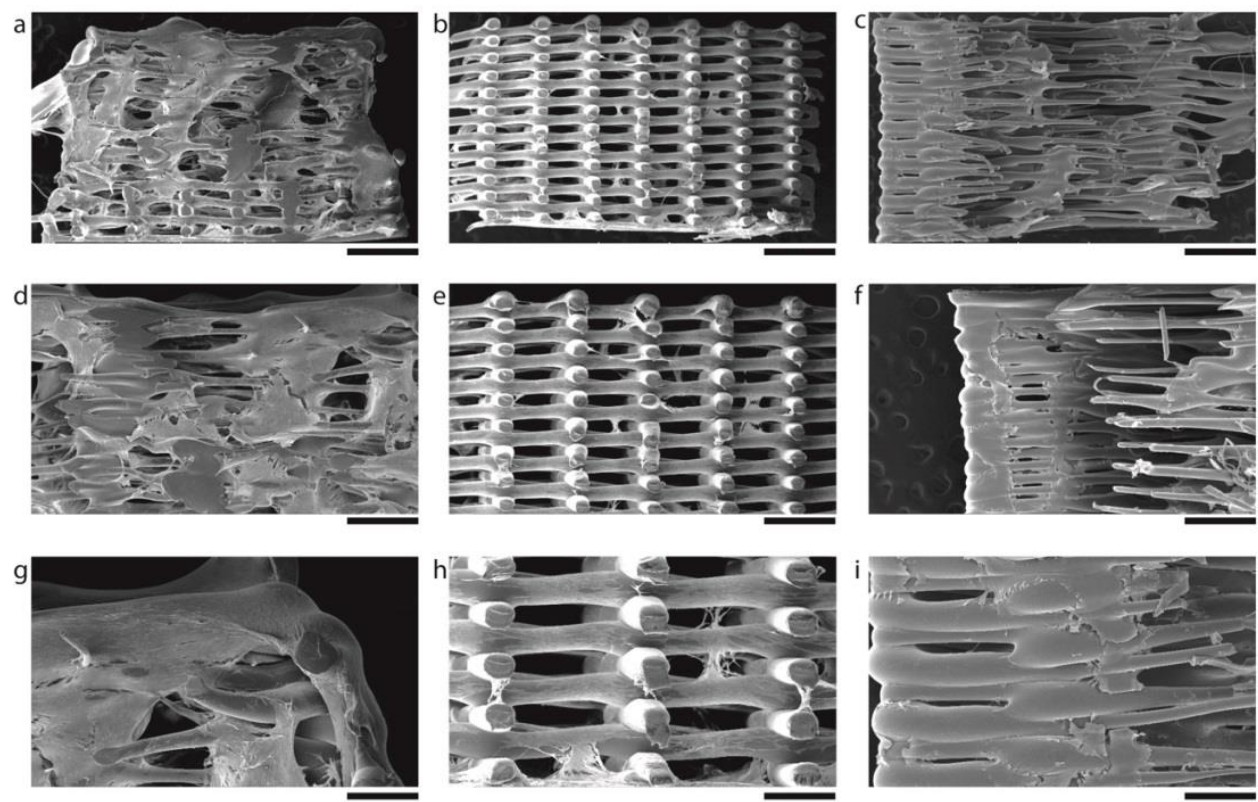

Figure 3. ECM production at day 28 in basic medium. A full view and close-up of $G(a, d, g)$, NG 90 (b, e, h) and NG 15 (c, f, i) scaffolds, respectively, is displayed at increasing magnifications. Despite the absence of differences in cell number after 28 days, the gradient constructs showed an increased ECM deposition, probably due to an increased number of fiber contact points. Scale bar $1 \mathrm{~mm}(\mathrm{a}-\mathrm{f}), 500 \mu \mathrm{m}(\mathrm{g}-\mathrm{i})$.

\section{3. hMSC differentiation}

Under chondrogenic conditions the structural pore shape gradient of the scaffolds was correlated with an enhanced expression of chondrogenesis related genes after 14 days (Figure 4a). Aggrecan and collagen II alpha were 4 to 5 times higher compared to the controls, whereas Sox-9 displayed a 3-fold increase with respect to NG15. In terms of biochemical analysis, no major differences could be seen for GAGs synthesis among the groups at the same time point, even though there was a significant increase of GAGs production during culture under chondrogenic conditions (Figure 5a). Collagen type II production was also increased in G scaffolds after 2 weeks (Figure S5). Under basic conditions, as expected, the 
increase in GAG amount was less pronounced. During the first 2 weeks, GAGs production increased significantly, while in the last 2 weeks it remained constant.

Under osteogenic conditions no major differences could be seen among osteogenesis related genes for the different constructs, with the exception of BMP2 which was significantly increased in G scaffolds compared to NG15 and NG90 scaffolds (Figure 4b, Figure S4). Bone sialoprotein seemed to be significantly enhanced in NG90 with respect to NG15. The ALP activity didn't show any significant difference among the gradient scaffolds and controls at any time point (Figure 5b). Osteocalcin production was increased in NG15 and G scaffolds compared to NG90 scaffolds (Figure S5). hMSCs cultured in basic medium displayed an increase in ALP activity in the first seven days followed by a decrease during the rest of the study.
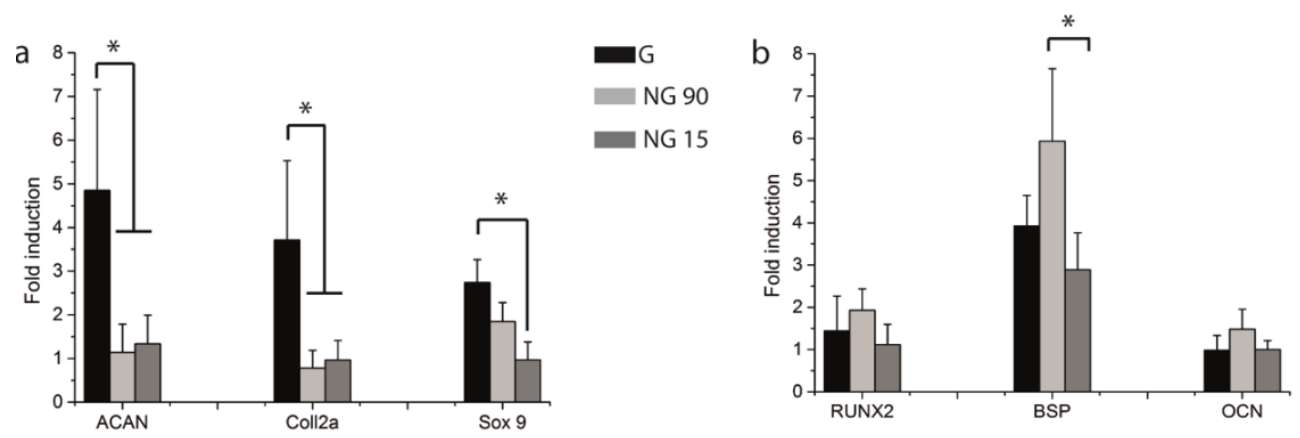

Figure 4. Fold increase of chondrogenic (a) and osteogenic (b) markers under chondrogenic and osteogenic conditions respectively. Under chondrogenic conditions the gradient greatly enhanced the expression of chondreogenic markers in comparison to the controls. Under osteogenic conditions the NG 90 scaffolds enhanced the expression of BSP after 14 days. The fold induction was normalized by the gene expression of hMSCs cultured in gradient scaffolds in basic medium. ${ }^{*} p<0,05$. 

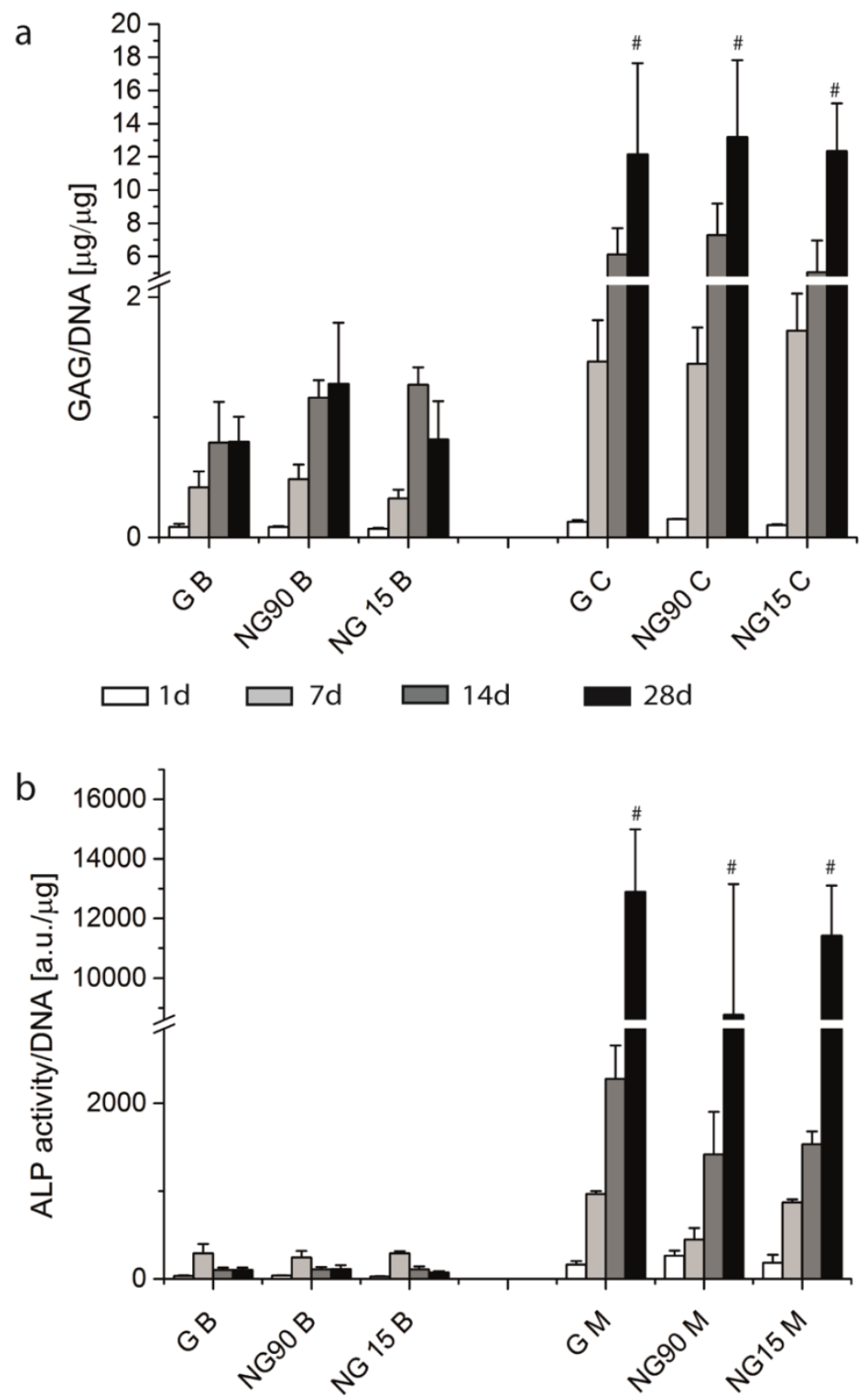

Figure 5. GAGs amount over time normalized by DNA amount (a) and ALP activity over time normalized by DNA amount (b). Under chondrogenic conditions an increase in GAGs was detected, even though no significant differences could be seen among the constructs within the same time point. Similarly, under mineralization conditions the ALP activity 
increase over time without significant differences among the $G$ and the NG scaffolds. \# shows statistical significance with respect to previous time points, $p<0.05$.

\section{4. hMSC differentiation within the gradient zones}

To better understand if any difference is present among the cells residing in the different gradient zones, a partition analysis was performed after 14 and 28 days of differentiation, followed by gene expression analysis and biochemical analysis respectively. Chondrogenic markers displayed a significant difference among the rhomboidal region and the squared pore region, in which the genes were overexpressed (Figure 6a). The same trend was shown by the biochemical analysis in terms of GAGs synthesis (Figure 7a). The amount of GAG deposited was normalized by the cell density intended as DNA amount per pore volume (Figure S6a) and by the pore volume only (Figure S6b). The trend remained unvaried also when the pore volume was considered in the analysis, thus suggesting that the observed enhanced differentiation is dependent only from the pore shape. Under osteogenic conditions an opposite trend with respect to the chondrogenic one could be observed, in which the rhomboidal shapes enhanced, the expression of osteogenic differentiation related genes (Figure 6b). Also ALP activity (Figure $7 \mathrm{~b}$ ) was significantly higher in the rhomboidal pores (0-30/15) with respect to the other zones.

The culture of gradient structures in medium containing both osteogenic and chondrogenic soluble signals resulted in an increased of GAGs deposition (Figure 7c) in the squared pore region (0-90) and of ALP (Figure 7d) activity in the rhomboidal pore (0-30/0-15) shaper zone, thus confirming the results obtained in single differentiation medium. As displayed in Figure S7a and b, the trend did not vary when the structural component was considered for chondrogenic analysis. A possible reason for the observed trends could be due to the local variations in nutrients availability, in particular oxygen, which could result in different degree of hypoxia in the gradient scaffolds regions. To test this hypothesis, hypoxia inducible factor (HIF)-1 $\alpha$ and HIF-2 $\alpha$ were measured (Figure S8), showing an increased expression in the more chondrogenic squared pore region. 

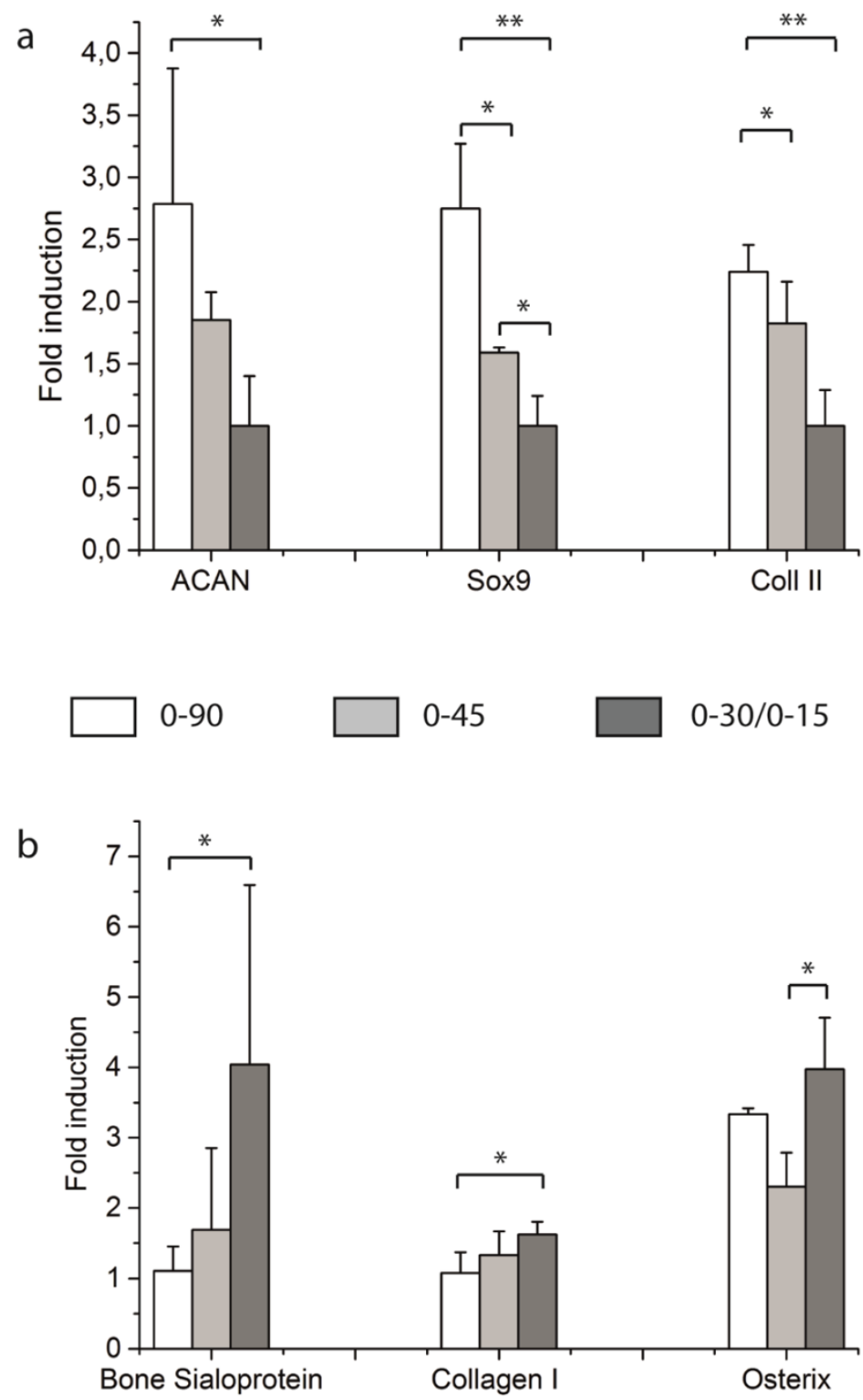

Figure 6. Fold increase of chondrogenic (a) and osteogenic (b) markers under chondrogenic and osteogenic conditions respectively after 28 days in the different gradient zones. Under chondrogenic conditions the squared shape gradient greatly enhanced the expression of chondrogenic markers. Under osteogenic conditions the trend was inverted. * Statistical significance with respect to the rhomboidal squared area, $p<0,05 ;{ }^{* *} p<0,01$. 


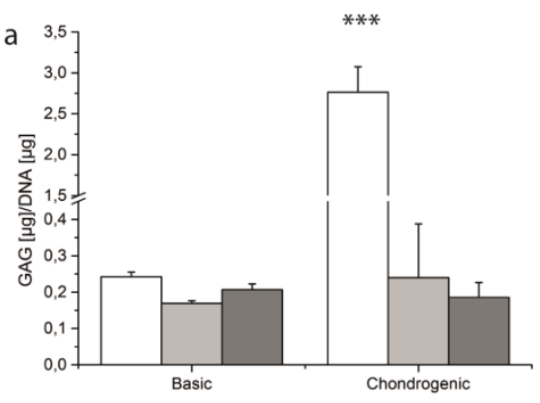

$0-90$

C

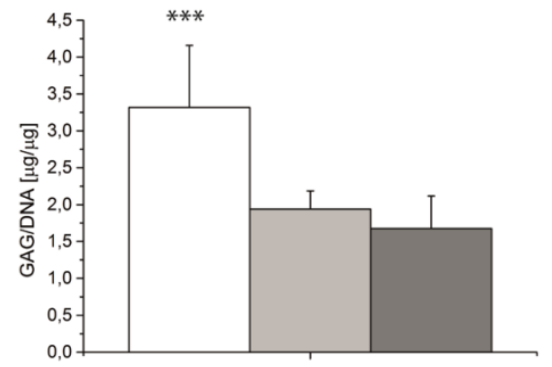

b

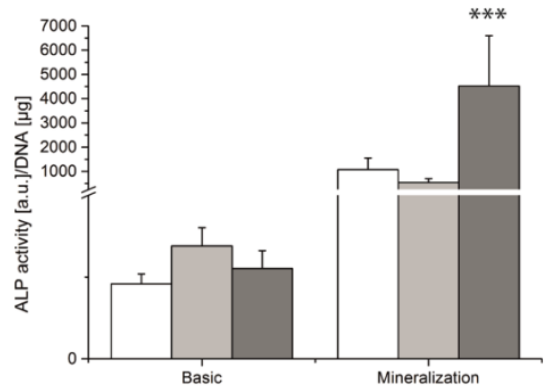

$0-45$

$0-30 / 0-15$
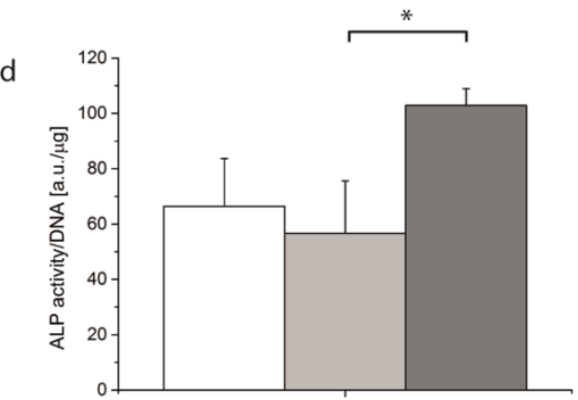

Figure 7. After 28 days, the ALP activity was significantly increased by the rhomboidal geometry (b) whereas the GAG deposition was greater in the squared pore area (a). GAGs (c) and ALP (d) analysis of gradient scaffolds cultured under osteochondral conditions. Briefly, the hMSCs within the scaffolds were cultured in a medium composed by chondrogenic and osteogenic medium in a 1:1 ratio. The opposite trend in differentiation was maintained. The rhomboidal pores supported a better ALP activity while the squared pores displayed a higher GAG deposition. * Statistical significance with respect to the rhomboidal squared area, $p<0,05 ;{ }^{* * *} p<0,001$.

\section{Discussion}

In this study, we presented a novel strategy to generate a structural gradient in pore size and shape in 3D scaffolds fabricated by AM, in which the fiber deposition pattern was changed every $0.9 \mathrm{~mm}$. The conventional plotting setting $0-a$ is determined by " 0 " as the starting direction of the layer and " $a$ " as the increase in the angle between the fibers of one layer and the subsequent one $[12,22]$. Conventional plotting has lead so far mainly to squared pores in the 0-90 pattern and honeycomb like pore shape in the other patterns, in which pores are defined by several fibers from different layers [32]. In this study, the pattern 0-a-0-a was used (for example $0-45-0-45$ ) in order to have a pore defined by 2 subsequent 
layers and have a constant pore shape for 6 layers $(0.9 \mathrm{~mm})$. The variation in fiber deposition pattern determined a variation in the pore geometry and size, where decreasing the angle of deposition resulted in an increased rhomboidal shape and a consequent increase in pore perimeter, area, and volume. The increase was not continuous, since it was a discrete gradient in which 4 zones could be identified, whereas the control scaffolds showed the same pore geometry and size along the longitudinal axis. The scaffolds were made by PCL, since this material was already applied in surgery and clinical trials are currently ongoing [33, 34]. When considering the mechanical analysis of the fabricated scaffolds, different deformations or slopes between the loading and unloading phases highlighted the characteristic damping effects at corresponding response times. Different reductions in the stiffness compared to a solid cylinder arise from the complex order and geometry of the AM scaffolds. Thus, the angle variations in the layer deposition led to variations in the mechanical response, which is dominated by the interactions of individual strands within the regular ordered shapes of the scaffolds. The mechanical behavior can be understood and explained from structural mechanics, where the layer dimensions and directions have to be taken into account.

In literature, the improved cells seeding efficiency in plotted scaffold showing a gradient in porosity has been already reported [31]. In our constructs no major differences could be seen among the samples in which the cell seeding efficiency was within the $60-80 \%$ range. Even though the cell number profile was the same in the gradient constructs and controls, enhanced ECM deposition could be seen in gradient scaffolds. The geometry seemed not to affect the cell number after seeding or the hMSC proliferation trend (Figure 2). In control scaffolds cells covered the fibers and in the $0-90$ configuration bridged the fibers through the pores, whereas in gradient scaffolds ECM was present all over the structure independently from the pore geometry. Bidan et al. observed different pore closure patterns of scaffolds seeded with MC3T3-E1 pre-osteoblasts depending on the pore geometry. Pore closure began at the edges and moved toward the center of the pore [21]; in this study, the increased number of fiber connections of the gradient scaffolds might have brought to a faster and higher pore closure with 
respect to the controls, probably due to an increased number of fiber connections where the pore closure could begin.

Several scaffolds based on PLGA microspheres were studied, which presented a gradient in order to promote hMSC differentiation (i.e. stiffness and growth factors) $[30,35,36]$; the solely pore geometry was never studied as a cue supporting hMSC differentiation. After 14 days of culture, we showed that the chondrogenic markers aggrecan, sox9 and collagen Ila were significantly upregulated with respect to the non-gradient scaffolds. Despite a continuous increase in GAGs amount in all the scaffolds tested with culture time, evidencing the correct chondrogenic differentiation of hMSCs in presence of soluble factors, differences among gradient and non-gradient scaffolds were not visible at a bulk biochemical level when measuring GAGs deposition.

No major differences were shown by hMSCs growing in gradient or non-gradient scaffolds in relation to early gene osteogenic markers such as runx2 and late markers such as osteocalcin after 14 days. Also at the protein level no major differences were shown between the ALP activity of cells cultured in G and NG scaffolds. According to Yilgor et al., a link between the geometry of the construct and the osteogenic differentiation of hMSCs might be established and the scaffold geometry could be optimized to improve hMSC osteogenic differentiation [37]. Our findings are in line with this hypothesis. In Yilgor's study, PCL scaffolds made with different architectures were analyzed. It was shown that the 0-90 conformation with an offset of $380 \mu \mathrm{m} \pm 20 \mu \mathrm{m}$ (the size of 1 fiber) displayed the highest ALP levels compared to other scaffolds with $0-90$ and $0-45$ angle deposition. During the culture time here analyzed, the ALP levels started to increase after 7 days from the beginning of the differentiation and continued to increase until 28 days, which is in line with what can be found in literature for PCL scaffolds [38, 39].

To investigate the differences between the hMSCs residing in each pore geometry region within the gradient scaffold, a partition analysis was performed. After 14 days of culture, aggrecan, sox 9 and collagen type lla presented a 3-fold induction in squared pores (0-90 design) and 2-fold increase in the 0-45 pattern with respect to the 0-30/0-15 patterns. The trend was maintained after 28 days of differentiation at the biochemical level, since GAG/DNA amount was significantly higher in the 
squared pores compared to rhomboidal pores. An opposite trend was found in literature in studies correlating the behavior of chondrocytes in 3D plotted scaffolds displaying a pore-size gradient both at the genetic and biochemical levels [40], where genetic and biochemical markers increased with increasing the pore size. Similarly, Oh et al. showed an increase of chondrogenic markers expression such as sox-9 and collagen type lla from small pores (90-105 $\mu \mathrm{m})$ to larger pores (370$400 \mu \mathrm{m})$ in adipose derived stem cells [41]. Our pore dimensions increased from the squared-pore to the rhomboidal ones. The squared pore size is defined by the side, while each rhomboidal pore size is defined by the minor diagonal (d), which did not vary significantly, and the major diagonal (D), which varied greatly. Squared pores had a dimension of $560 \mu \mathrm{m} \pm 30 \mu \mathrm{m}$ in size, the $0-45$ plotting pattern generated pores with a $d$ of $500 \mu \mathrm{m} \pm 40 \mu \mathrm{m}$ and a $\mathrm{D}$ of $1350 \mu \mathrm{m} \pm 90 \mu \mathrm{m}$, whereas the 0-30 and 0-15 showed a d of $540 \mu \mathrm{m} \pm 40 \mu \mathrm{m}$ and $470 \mu \mathrm{m} \pm 20 \mu \mathrm{m}$ and $\mathrm{a} D$ of $1970 \mu \mathrm{m} \pm 80 \mu \mathrm{m}$ and $3010 \mu \mathrm{m} \pm 130 \mu \mathrm{m}$, respectively. The height of the pores remained constant at $140 \mu \mathrm{m} \pm 10 \mu \mathrm{m}$. In our scaffolds, the smallest pores were bigger than the larger pores of the Oh's study and the trend observed was opposite. The different range in pore size and the effect of the pore shape might explain this discrepancy. Several studies [21, 42, 43] proved that the pore shape can influence ECM deposition, cellular cytoskeleton architecture and speed of pore closure. In this study, both pore size and shape changed along the $z$ axis. GAGs are one of the major components of chondrocyte-deposited ECM. To distinguish between the influence of cellular density and scaffold architecture on GAGs formation, we normalized by DNA amount and by pore volume (Figure S7), respectively. The pore size was included by normalizing the GAG amount by the cell density intended as DNA amount per volume unit. Results displayed the same trend with the same statistical significance, defining the pore shape as the main justification for the different behaviour of hMSCs residing in the compartment of the G scaffolds.

An opposite trend was found for hMSC osteogenic differentiation. At the genetic level a trend could be seen for bone sialoprotein, collagen type I and osterix, suggesting an enhanced osteogenic differentiation in the rhomboidal pores compared to the squared ones. The results were confirmed by the biochemical 
analysis that showed an increased ALP activity in the rhomboidal 0-30/0-15 pore geometry when compared to the $0-45$ rhomboidal or the $0-90$ squared pore geometry. In literature there are no studies, to the best of our knowledge, relating pore shape to hMSC osteogenic differentiation that, in accordance to some studies $[9,44,45]$, is not affected by the overall porosity of the construct. In this respect, the overall porosity of the gradient zones is the same, therefore it could be concluded that the differences present within the gradient scaffolds may be attributed to the variation in pore shape and size. In case of ALP, we didn't dissect the specific role of pore size by that of pore shape by normalizing for pore volume as we did for GAG deposition, because the ALP test measures the activity of the protein and not its production.

It was already proved that by supplementing the medium with soluble factors stimulating the osteogenic and chondrogenic hMSC differentiation simultaneously, both lineages will be generated in culture [46]. To further confirm the correlation between pore shape and the differences in hMSC differentiation lineages, gradient scaffolds were seeded with hMSCs and cultured in a medium providing differentiation signal toward the osteogenic and the chondrogenic lineage. To do so, the osteogenic and chondrogenic medium were combined in a 1:1 ratio. The results further confirmed the findings of our previous tests. Rhomboidal pores displayed a significantly higher ALP activity, whereas the highest amount of GAGs was located in the squared pores. The effect of pore shape was confirmed with the normalization by cell density (Figure S7).

A further possible explanation for the observed influence of pore shape gradients could be due to the local differences in oxygen availability to the cells. As in developmental biology it is known that in presence of hypoxia chondrogenesis is facilitated, we reasoned that in these pore shape gradient scaffolds a change of pore shape could result in a change of local oxygen available, thus affecting the expression of hypoxia inducible factors. In particular, HIF-1 $\alpha$ and HIF-2 $\alpha$ were measured. Hypoxia-inducible factors, including HIF-1 $\alpha$ and HIF-2 $\alpha$ induce glycolysis and angiogenesis in order to try restoring oxygen homeostasis. Hypoxiainducible expression of some genes, such as Glut-1, p53, p21 or Bcl-2, is dependent on HIF-1 $\alpha$ translocation to the cell nucleus. HIF-2 $\alpha$ regulates the 
vascular endothelial growth factor (VEGF) expression and its expression levels influence the relative contribution of aerobic and anaerobic cellular metabolism. Results showed, indeed, an increase of HIF-1 $\alpha$ and HIF-2 $\alpha$ with decreasing the pore rhomboidal geometry from $0-15$ to $0-90$ degrees (Figure S8), corresponding to the increased chondrogenesis observed by genetic and protein analysis.

The combination of the pore shape gradient in combination with pre-differentiated cells in a pre-osteochondral construct may be considered as future perspective for the improved outcome of implants for osteochondral lesion treatment. According to our findings, gradient scaffolds should be implanted with the squared pores on the chondral side and the rhomboidal on the bone side. Further studies in animal models aiming to a better understanding if such behavior is maintained in vivo are needed. Additional research should be aimed at the analysis of the ECM composition in order to understand to which extent the construct mimics the anatomical and molecular transition from the subchondral bone to the chondral side in vivo. From a biofabrication perspective, it would be very interesting to show whether different pore size and shape gradients would result in a similar influence on stem cell activity in combination with soluble factors. In this respect, other AM technologies like stereolithography might be also considered, as this would allow to expand the geometrical features of the fabricated pore network. Melchels et al., for example, showed the creation of sinusoidal-like pore network patterns and how varying such network resulted in better cell seeding efficiency [47]. The palette of biomaterials could be also expanded to determine if the observed effects are independent from the material chemistry or, more likely, can be further modulated by it.

In the past years microfracture technique was applied as surgical treatment of osteochondral lesions [48], its application in combination with scaffolds displayed an enhanced healing of osteochondral defects [49]. A possible study aiming at clinical translation, for example, could foresee the use of our gradient scaffolds in an orthotopic osteochondral defect with the squared pores facing the cartilage side and the rhomboidal ones facing the bone side, as previously suggested, where MSCs could be retrieved from the bone marrow through microfracture procedures. Practical examples where such scaffolds could be used in osteochondral locations 
comprise defects in long bone joints such as knees, hips, and ankles, which are typically affected more frequently from traumatic accidents or ageing.

\section{Conclusion}

In this study a construct produced via additive manufacturing and presenting a gradient in pore shape was produced and its effect on hMSC proliferation and differentiation was analyzed. A new fiber deposition pattern was presented, allowing the generation of pores of different shapes within the same construct. The 0-90 fiber alignment generated squared pores that supported enhanced differentiation of hMSCs toward the chondrogenic lineage, as shown by GAGs deposition and the expression of chondrogenic related genes. The variation in the fiber deposition pattern led to an increasing rhomboidal pore conformation by switching to $0-45,0-30$ and $0-15$ angle depositions. The most rhomboidal pore geometry sustained enhanced ALP activity and osteogenic related genes expression with respect to the other gradient zones. This trend was maintained when the gradient scaffold was cultured in a medium supporting both osteogenic and chondrogenic differentiation. The study confirmed that the pore shape can play a role as a modulator of cell differentiation in vitro.

\section{Experimental Section}

Scaffold preparation: Scaffolds were fabricated via additive manufacturing with a Bioscaffolder (SysENG, Germany). Scaffolds made of poly( $\varepsilon$-caprolactone) (PCL, Sigma-Aldrich, USA) were produced. Briefly the polymer was placed in a stainless syringe and processed at $100{ }^{\circ} \mathrm{C}$. The application of 5 bars of pressure via a nitrogen flow from a pressurized cap drove the molten polymer through an extrusion screw rotating at $200 \mathrm{rpm}$.

During plotting, the needle diameter, layer thickness, fiber spacing and speed were kept constant at $200 \mu \mathrm{m}, 150 \mu \mathrm{m}, 800 \mu \mathrm{m}$ and $180 \mathrm{~mm} / \mathrm{min}$, respectively. The fiber deposition pattern was kept constant at 0-90 (NG 90) and 0-15 (NG 15) for the control scaffolds, while the gradient scaffold was plotted by varying the fiber deposition pattern every millimeter from $0-90$ to $0-45,0-30$, and $0-15$. The scaffolds were plotted as cylinders of $6 \mathrm{~mm}$ in diameter and $3.6 \mathrm{~mm}$ in height. 
Mechanical analysis: Compression tests were conducted using a rheometer (Discovery Hybrid Rheometer DHR-1, TA Instruments, US), which is equipped with a force rebalanced transducer (FRT) for axial forces up to $50 \mathrm{~N}$ and sensitivity of $0.01 \mathrm{~N}$. Cylindrical specimens of about $4 \mathrm{~mm}$ in height were placed in between parallel metal plates of $25 \mathrm{~mm}$ in diameter. The vertical gap height and axial force were recorded at $37{ }^{\circ} \mathrm{C}$ during controlled deformation speed experiments with 1 $\mu \mathrm{m} / \mathrm{s}$ up to maximum forces of $1 \mathrm{~N}$ as well as $10 \mathrm{~N}$ and converted to an apparent compression strain. The unloading phase was also recorded with $1 \mu \mathrm{m} / \mathrm{s}$ in order to obtain information on the recovery behavior and possible hysteresis effects.

Cell expansion and culture: hMSCs (male, age 22) were retrieved from the Institute of Regenerative Medicine (Temple, Texas). Briefly, a bone marrow aspirate was drawn and mononuclear cells were separated using density centrifugation. The cells were plated to obtain adherent hMSCs, which were harvested when cells reached $60-80 \%$ confluence. These were considered passage 0 (P0) cells. These P0 cells were expanded, harvested and frozen at passage 1 (P1) for distribution. Cells were grown in MSC proliferation medium, which contains minimal essential medium (a-MEM, Life Technologies, Bleiswijk, the Netherlands) supplemented with $10 \%$ fetal bovine serum (FBS, Lonza), $100 \mathrm{U} / \mathrm{ml}$ penicillin (Life Technologies, Bleiswijk, the Netherlands), $10 \mu \mathrm{g} / \mathrm{ml}$ streptomycin (Life Technologies, Bleiswijk, the Netherlands), $2 \mathrm{mM}$ L-glutamine (Life Technologies, Bleiswijk, the Netherlands), $0.2 \mathrm{mM} \mathrm{L-ascorbic} \mathrm{acid} \mathrm{2-phosphate} \mathrm{magnesium} \mathrm{salt} \mathrm{(ASAp,} \mathrm{Sigma-}$ Aldrich, Zwijndrecht, The Netherlands) and $1 \mathrm{ng} / \mathrm{ml}$ of basic fibroblast growth factor-2 (bFGF-2, Fisher Scientific, Landsmeer, the Netherlands) at $37^{\circ} \mathrm{C}$ in a humid atmosphere with $5 \% \mathrm{CO}_{2}$. Cells were expanded up to approximately $80 \%$ confluency and either frozen for further use or seeded on the scaffolds.

Cell seeding on scaffolds: Cells were seeded under static condition within the $G$ and NG scaffolds. Briefly, after trypsinization with $0.25 \%$ trypsin (Life Technologies, Bleiswijk, the Netherlands), cells (passage 2-4) were counted using a Bückner chamber and re-suspended in proliferation medium at a density of 500'000 cells in $40 \mu \mathrm{L}$. The day before seeding, scaffolds were disinfected in $70 \% \mathrm{EtOH}$ for $30 \mathrm{~min}$ under stirring, washed 3 times in phosphate buffered saline solution (PBS) (Lonza, Breda, the Netherlands), and incubated overnight in cell proliferation medium to 
allow protein adsorption on the scaffold's fibers. After protein adsorption, the $40 \mu \mathrm{L}$ of cell suspension were placed on the scaffold in a drop wise fashion to account for a cell seeding density of 500'000 cells/scaffold. The seeded scaffolds were placed for 4 hours in the incubator to allow cell adhesion before adding the cell culture medium.

Cells were cultured on the $G$ and NG scaffolds for 7 days in proliferation medium. At day 7 , the proliferation medium was changed and the cells within the scaffolds were cultured for another 28 days in basic medium, mineralization medium consisting of basic medium supplemented with $10 \mathrm{nM}$ dexamethasone (SigmaAldrich Zwijndrecht, The Netherlands) and $10 \mathrm{mM} \beta$-glycerol-phosphate (SigmaAldrich, Zwijndrecht, The Netherlands), and chondrogenic medium consisting of DMEM supplemented with $50 \mathrm{mg} / \mathrm{mL}$ ITS-premix (Bexton Dickinson), $0.4 \mathrm{mM}$ Proline (Sigma-Aldrich, Zwijndrecht, The Netherlands), $50 \mathrm{mg} / \mathrm{mL}$ Ascorbic acid (ASAp, Sigma-Aldrich, Zwijndrecht, The Netherlands), $100 \mathrm{mg} / \mathrm{mL}$ sodium pyruvate (Sigma-Aldrich, Zwijndrecht, The Netherlands), $100 \mathrm{U} / \mathrm{ml}$ penicillin (Life Technologies, Bleiswijk, the Netherlands), $10 \mu \mathrm{g} / \mathrm{ml}$ streptomycin (Life Technologies, Bleiswijk, the Netherlands), $10 \mathrm{ng} / \mathrm{mL}$ transforming growth factor $\beta 3$ (TGF-ß3) (Life Technologies, Bleiswijk, the Netherlands) and $10^{-7} \mathrm{M}$ dexamethasone (Sigma-Aldrich, Zwijndrecht, The Netherlands). For the culture of the $G$ scaffolds under osteochondral conditions osteogenic and chondrogenic medium were mixed in a ratio 1:1 immediately prior the medium change. The medium was refreshed every 2 days.

Biochemical assays: The cell number per scaffold was calculated from the $\mu \mathrm{g}$ of DNA, obtained by a Cyquant DNA assay kit (Life Technologies, Bleiswijk, the Netherlands). Briefly, each scaffold was cut to improve lysis efficiency and freezethawed 5 times. After the freeze-thawing process, cells within the scaffolds were lysated by diluting the 20x lysis buffer provided with the kit using a saline buffer $(180 \mathrm{mM} \mathrm{NaCl}, 1 \mathrm{mM}$ EDTA in distilled water). After $1 \mathrm{~h}$ of lysis, samples were sonicated 2 times for 10 seconds using a Branson sonifier 250 (Emerson Industrial Automation, USA). DNA content was quantified with a CyQuant kit (Life Technologies, Bleiswijk, the Netherlands) according to manufacturer's protocol and fluorescence was measured at $480 \mathrm{~nm}$ using a spectrophotometer LS50B (Perkin 
Elmer, The Netherlands). DNA concentrations were calculated from a $\lambda$ DNA standard curve.

To evaluate hMSC differentiation toward the osteogenic lineage, alkaline phosphatase (ALP) activity was measured using a CDP star kit (Roche, Almere, The Netherlands). For this purpose, $10 \mu \mathrm{L}$ of sample was added to a well of a white 96-well plate and $40 \mu \mathrm{L}$ of substrate was added. After 15 minutes incubation, luminescence was read using a spectrophotometer LS50B (Perkin Elmer, The Netherlands). ALP activity was corrected for DNA content.

To evaluate the differentiation toward the chondrogenic lineage, glycosaminoglycans (GAGs) amount was quantified using 1,9-Dimethyl Methylene Blue (DMMB) assay. Specifically, $25 \mu \mathrm{L}$ of sample were placed into a transparent flat bottom 96 well plate and $5 \mu \mathrm{L}$ of $2.3 \mathrm{M} \mathrm{NaCl}$ solution were added. Then, $150 \mu \mathrm{L}$ of DMMB solution were added and absorbance was read using a Multiscan Go (Fisher Scientific, Landsmeer, the Netherlands) plate reader at a wavelength of $525 \mathrm{~nm}$. GAGs content was quantified with a chondroitin standard curve and corrected for DNA content.

Osteocalcin $(\mathrm{OCN})$ and collagen type-II hMSC production was analysed after 14 days of culture using ELISA (human osteocalcin and collagen type II ELISA kits, Invitrogen). The test was performed on 5 samples per condition. In short, the medium was removed and samples were washed using ice cold PBS. A buffer was prepared using 890 volumes of miliQ water, 100 volumes of RIPA buffer (Cell Technologies) and 10 volumes of Halt ${ }^{\mathrm{TM}}$ protease and phosphatase inhibitor (Thermo Scientific). 150 microliters of the buffer was added to each samples and the samples were incubated on ice for 10 minutes. The buffer was then collected from the samples and centrifuged at $11000 \mathrm{~g}$ for 15 minutes. The supernatant was collected and used for the ELISA tests which were performed according to manufacturer's protocol. Briefly, $25 \mu \mathrm{l}$ of samples and standard solutions were added to the osteocalcin and collagen type II antibody-coated strip- well plates. $100 \mu \mathrm{l}$ of Anti-OST-HRP and $100 \mathrm{ul}$ of Anti-Col2-HRP conjugates, respectively, were added to each correspondent well, and the plates were covered and incubated for 2 hours at room temperature. After incubation, the solutions in the wells were aspirated and the wells were washed 
3 times using a washing solution provided in the kit. Then, $100 \mu \mathrm{l}$ of a chromogen solution (Tetramethylbenzidine) was added to each well and the plates were incubated for 30 minutes at room temperature in the dark. Finally, $100 \mu \mathrm{l}$ of stop solution was added to each well. The optical density of each well was read at $450 \mathrm{~nm}$ using a plate reader (MULTISKAN GO, Thermo Scientific). A standard curve was plotted in Microsoft excel and concentration of OCN and collagen type II were determined in each well according to the standard curves. Western Blot analysis: hMSC passage 2-4 were cultured in normoxic (21\% oxygen) conditions on both petri dishes and AM scaffolds, and in hypoxic (2,5\% oxygen) conditions on petri dishes only (positive control). The cells were lysed in radioimmunoprecipitation assay (RIPA) lysis buffer (sc-24948, SantaCruz Biotechnology), and the protein content was determined using Pierce BCA protein assay kit (\#23227, Thermo Scientific Pierce), with bovine serum albumin as the standard. Each sample was mixed with 4x Laemmli Sample Buffer (\#161-0737, BioRad) under denaturing conditions. $20 \square \mathrm{g}$ of protein sample were loaded per lane and resolved on a 4-20\% precast polyacrylamide gel (\#456-8094, BioRad). Then, proteins were transferred to a PVDF membrane for immunoblotting. After blocking $1 \mathrm{~h}$ at room temperature with $5 \%$ BSA in Tris-buffered saline containing 0.1\% Tween-20 (Santa Cruz Biotechnology), the membranes were incubated overnight at $4^{\circ} \mathrm{C}$ with HIF-1a (28b) antibody (\#sc-13515, Santa Cruz Biotechnology), or HIF-2 $\alpha$ antibody (\#sc-46691, Santa Cruz Biotechnology), diluted 1:250 in Tris-buffered saline containing 0.1\% Tween-20). GAPDH antibody (\#sc365062, Santa Cruz Biotechnologies) diluted 1:2500 was used as loading control for normalization. Membranes were washed with Tris-buffered saline, and incubated with horseradish peroxidase (HRP)-conjugated secondary antibodies (DAKO) diluted 1:2500 in Tris-buffered saline containing 0.1\% Tween-20 for $45 \mathrm{~min}$ at room temperature. After washing, blots were developed using Clarity Western ECL Substrate (\#1705060, BioRad) and the HRP activity of the blots were imaged using a BioRad Chemidoc Touch imager. Finally, blots were semi-quantified using ImageJ.

Microscopy analysis: $\mathrm{G}$ and NG scaffolds were analyzed by scanning electron microscopy (SEM, Philips - XL 30 ESEM-FEG). Directly after plotting, scaffolds 
were cut in half, gold sputtered and analyzed. SEM images were analyzed using Image $\mathrm{J}$ software in order to measure the fiber diameter, fiber spacing, and pore dimensions.

Scaffolds cultured in basic medium for 28 days were fixed using $10 \%$ formalin, dehydrated by an increased series of ethanol concentration (50-60-70-80-90-96$100 \%$ ) and cut in half. The final dehydration step was carried out via immersion in Hexamethyldisilazane (Sigma-Aldrich, Zwijndrecht, The Netherlands) and overnight evaporation. Dry scaffolds were mounted on SEM stubs, gold sputtered (Cressington sputter coater 108 auto), and analyzed using $10 \mathrm{kV}$ and a working distance of $25 \mathrm{~mm}$.

Gene expression analysis: For gene expression analysis, the scaffolds were taken from the medium, washed twice with PBS, cut into small pieces, placed in an Eppendorf tube containing $750 \mu \mathrm{L}$ of TRIzol $\AA^{8}$ (Invitrogen), and stored at $-80^{\circ} \mathrm{C}$. In the case of partition analysis the gradient scaffolds were cut in order to separate the gradient zones and the 3 samples were located in the same vial prior to the addition of TRIzol $\Theta$, in order to ensure the collection of enough RNA. RNA isolation was performed by using a Bioke RNA II nucleospin RNA isolation kit (MakereyNagel). $150 \mu \mathrm{L}$ of $\mathrm{CHCl}_{3}$ were added and the vials were vigorously mixed, followed by a centrifugation at $12000 \mathrm{~g}$ for 15 minutes at $4 \stackrel{\circ}{\circ}$. The aqueous phase was transferred into a new tube and an equal amount of $70 \%$ ethanol was added. The mixture was transferred into a filter columns from the kit and the extraction was carried on by following the manufacturer's protocol. RNA concentration and purity was evaluated via an ND1000 spectrophotometer (Nanodrop Technologies, USA); cDNA was synthetized using iScript ${ }^{\mathrm{TM}}$ (BIO-RAD) according to the manufacturer's protocol. Quantitative polymerase chain reaction (qPCR) was performed on the obtained cDNA by using the $\mathrm{iQ}$ SYBR® Green Supermix (BIO-RAD) and the primers listed in Table S1, in supporting information. PCR reaction was carried out on the MyiQ2 Two-Color Real-Time PCR Detection System (BIO-RAD) under the following conditions: the $\mathrm{cDNa}$ was denatured for 10 minutes at $95 \stackrel{\circ}{\circ}$, followed by 45 cycles, consisting of 15 seconds at $95^{\circ} \mathrm{C}, 15$ seconds at $60 \stackrel{\circ}{\circ}$ and 15 seconds at $72{ }^{\circ} \mathrm{C}$. A melting curve was generated from each reaction to test the presence of primer dimers and a specific product. The cycle threshold was calculated by the 
Bio-Rad iQ5 optical system software, in which the threshold was set in the lower log-linear region of the fluorescent signal. Ct values were normalized by the B2M housekeeping gene and $\Delta \mathrm{Ct}$ ((average of $\mathrm{Ct}$ control)-Ct value). Results were expressed as fold induction in mRNA expression normalized to the gene expression of the gradient scaffolds cultured in basic medium. In the partition analysis, the relative RNA expression was normalized by the squared pore region $(0-90)$ for the osteogenic analysis and the rhomboidal region (0-30/15) for the chondrogenic analysis.

Statistical analysis: All the quantitative data are expressed as mean \pm standard deviation. Statistics were performed using IBM SPSS Statistics 20. A two-way ANOVA with Tukey as post-hoc test were used. Statistical significance between the control group and the experimental groups is indicated with $\left(^{*}\right)$ which represents a $p$-value $<0.05,\left({ }^{* *}\right)$ which represents a $p$-value $<0.01$, and $\left({ }^{* * *}\right)$ which represents a $p$-value $<0.001$.

\section{Acknowledgements}

This research was financially supported by the Technology foundation STW (STW, 11135) and by the Province of Limburg. The experimental support of N. Leone in mechanical testing is greatly appreciated. 


\section{References}

[1]. R. Langer, J.P. Vacanti, Science 1993, 260, 920.

[2]. B.M. Abdallah, M. Kassem, Gene Ther. 2008, 15, 109.

[3]. F.P.W. Melchels, Ferry M.A.N. Domingos, T.J. Klein, J. Malda, J.P. Bartolo, D.W. Hutmacher, Prog. Polym. Sci 2012, 37, 1079.

[4]. W.L. Grayson, F. Zhao, B. Bunnell, T. Ma, Biochem. Biophys. Res. Commun. 2007, 358, 948.

[5]. S.H. Hsu, C.T. Chen, Y.H. Wei, Stem Cells 2013, 31, 2779.

[6]. A.J. Engler, S. Sen, H.L. Sweeney, D.E. Discher, Cell 2006, 126, 677.

[7]. S. Even-Ram, V. Artym, K.M. Yamada, Matrix control of stem cell fate. Cell 2006, 126, 645.

[8]. T. Kawano, M. Sato, H. Yabu, M. Shimomura, Biomater. Sci. 2014, 2, 52.

[9]. P. Kasten, I. Beyen, P. Niemeyer, R. Luginbuhl, M. Bohner, W. Richter, Acta Biomater. 2008, 4, 1904.

[10]. T.B. Woodfield, J. Malda, J. de Wijn, F. Peters, J. Riesle, C.A. van Blitterswijk, Biomaterials 2004, 25, 4149.

[11]. L.D. Harris, B.-S. Kim, D. J. Mooney, 1998.

[12]. D.W. Hutmacher, Biomaterials 2000, 21, 2529.

[13]. C.E. de Andrea, P.C. Hogendoorn, J. Pathol. 2012, 226, 219.

[14]. A. Di Luca, C.A. van Blitterswijk, L. Moroni, Birth Defects Res., Part C 2015, 105, 34.

[15]. K. Lavery, P. Swain, D. Falb, M.H. Alaoui-Ismaili, J. Biol. Chem., 2008, 283, 20948.

[16]. C.R. Nuttelman, M.C. Tripodi, K.S. Anseth, J. Biomed. Mater. Res. A 2006, 76, 183.

[17]. F. Barry, R.E. Boynton, B. Liu, J.M. Murphy, Exp. Cell. Res. 2001, 268, 189.

[18]. C. Phornphutkul, K.-Y. Wu, P.A. Gruppuso, Mol. Cell. Endocrinol. 2006, 249, 107.

[19]. I. Martin, S. Miot, A. Barbero, M. Jakob, D.J. Wendt, Biomech. 2007, 40, 75.

[20]. D. Schaefer, I. Martin, P. Shastri, R.F. Padera, R. Langer, L.E. Freed G. Vunjak-Novakovic Biomaterials 2000, 21, 2599.

[21]. C.M. Bidan, K.P. Kommareddy, M. Rumpler, P. Kollmannsberger, P. Fratzl, J.W. Dunlop, Adv. Healthc. Mater. 2013, 2, 186.

[22]. A. Berner, M.A. Woodruff, C.X. Lam, M.T. Arafat, S. Saifzadeh, R. Steck, J. Ren, M. Nerlich, A.K. Ekaputra, I. Gibson, D.W. Hutmacher, Int. J. Oral. Maxillofac. Surg. 2014, 43, 506.

[23]. T.B.F. Woodfield, C.A. van Blitterswijk, J. de Wijn, T.J. Sims, A.P. Hollander, J. Riesle, Tissue Eng., 2005, 11, 1297.

[24]. J. Genzer, J. Adhesion, 2005, 81, 417.

[25]. J. Genzer, Annu. Rev. Mater. Res. 2012, 42, 435.

[26]. A.M. Greiner, M. Jackel, A.C. Scheiwe, D.R. Stamow, T.J. Autenrieth, J. Lahann, C.M. Franz, M. Bastmeyer, Biomaterials 2014, 35, 611.

[27]. T.M. Keenan, A. Folch, Lab Chip 2008, 8,34.

[28]. C.H. Kuo, J. Xian, J.D. Brenton, K. Franze, E. Sivaniah, Adv. Mater. 2012, 24, 6059.

[29]. S.H. Oh, T.H. Kim, J.H. Lee, Biomaterials 2011, 32, 8254.

[30]. M. Singh, N. Dormer, J.R. Salash, J.M. Christian, D.S. Moore, C. Berkland, M.S. Detamore, J. Biomed. Mater. Res. A 2010, 94, 870.

[31]. J.M. Sobral, S.G. Caridade, R.A. Sousa, J.F. Mano, R.L. Reis, Acta Biomater. 2011, 7, 1009.

[32]. D.W. Hutmacher, T. Schantz, I. Zein, K.W. Ng, S.H. Teoh, K.C. Tan J. Biomed. Mater. Res. 2001, 55, 203.

[33]. M.A. Woodruff, D.W. Hutmacher, Prog. Polym. Sci. 2010, 35, 1217.

[34]. J.T. Schantz, T.C. Lim, C. Ning, S.H. Teoh, K.C. Tan, S.C. Wang, D.W. Hutmacher, Neurosurgery 2006, 58, ONS-E176.

[35]. X. Wang, E. Wenk, X. Zhang, L. Meinel, G. Vunjak-Novakovic, D.L. Kaplan, J. Control. Release 2009, 134, 81.

[36]. J.R. Tse, A.J. Engler, PLoS ONE, 2011, 6, e15978. 
[37]. P. Yilgor, R.A. Sousa, R.L. Reis, N. Hasirci, V. Hasirci, Macromol. Symp. 2008, 269, 92.

[38]. H.A. Declercq, T. Desmet, E.E. Berneel, P. Dubruel, M.J. Cornelissen, Acta Biomater. 2013, 9, 7699.

[39]. S.A. Park, S.H. Lee, and W.D. Kim, Bioprocess. Biosyst. Eng. 2011, 34, 505.

[40]. G. II, Im, J.-Y. Ko, and J.H. Lee,. Cell Transplant. 2012, 21, 2397.

[41]. S.H. Oh, T.H. Kim, G.II Im, J.H. Lee, Biomacromolecules 2010, 11, 1948.

[42]. P. Joly,G.N. Duda, M. Schone, P.B. Welzel, U. Freudenberg, C. Werner, A. Petersen, A. PLoS One 2013, 8, e73545.

[43]. J. Knychala, N. Bouropoulos, C.J. Catt, O.L. Katsamenis, C.P. Please, B.G. Sengers, Ann. Biomed. Eng. 2013, 41, 917.

[44]. M.E. Gomes, H.L. Holtorf, R.L. Reis, A.G. Mikos, Tissue Eng. 2006, $12,801$.

[45]. V. Karageorgiou, D. Kaplan, Biomaterials 2005, 26, 5474.

[46]. J. Li, S. Mareddy, D.M. Tan, R. Crawford, X. Long, X. Miao, Y. Xiao, Tissue Eng. Part. A 2009, 15, 2481.

[47]. F.P. Melchels, B. Tonnarelli, A.L. Olivares, I. Martin, D. Lacroix, J. Feijen, D.J. Wendt, D.W. Grijpma, Biomaterials 2011, 32, 2878.

[48]. J.R. Steadman, W.G. Rodkey, J.J. Rodrigo, Clin, Orthop. Relat. R. 2001, 391, S362.

[49]. H.A. Breinan, S.D. Martin, H.P. Hsu, M. Spector, J. Orthop. Res. 2000, $18,781$. 


\section{Supporting Information}

\begin{tabular}{|c|c|c|}
\hline Gene & Forward Primer & Reverse Primer \\
\hline B2M & ACAAAGTCACATGGTTCACA & GACTTGTCTTTCAGCAAGGA \\
\hline Aggrecan & AGGCAGCGTGATCCTTACC & GGCCTCTCCAGTCTCATTCTC \\
\hline Sox9 & TGGGCAAGCTCTGGAGACTTC & ATCCGGGTGGTCCTTCTTGTG \\
\hline Collagen Ila & CGTCCAGATGACCTTCCTACG & TGAGCAGGGCCTTCTTGAG \\
\hline Collagen la & GAGGGCCAAGACGAAGACATC & CAGATCACGTCATCGCACAAC \\
\hline Runx2 & TGGTTACTGTCATGGCGGGTA & TCTCAGATCGTTGAACCTTGCTA \\
\hline Osteocalcin & TGAGAGCCCTCACACTCCTC & CGCCTGGGTCTCTTCACTAC \\
\hline BSP & CCCCACCTTTTGGGAAAACCA & TCCCCGTTCTCACTTTCATAGAT \\
\hline Osterix & CCTCTGCGGGACTCAACAAC & AGCCCATTAGTGCTTGTAAAGG \\
\hline BMP-2 & ACTACCAGAAACGAGTGGGAA & GCATCTGTTCTCGGAAAACCT \\
\hline
\end{tabular}

Table S1. Primer list used for the differentiation analysis at genetic level. $\beta$-2- microglobulin (B2M) was used as housekeeping gene, aggrecan, SRY (sex determining region Y)-box 9 (sox 9) and collagen type II as chondrogenic markers and collagen type I, Runt-related transcription factor 2 (runx2), osteocalcin, bone sialoprotein (BSP), osterix, and bone morphogenetic protein-2 (BMP-2) as osteogenic markers. 


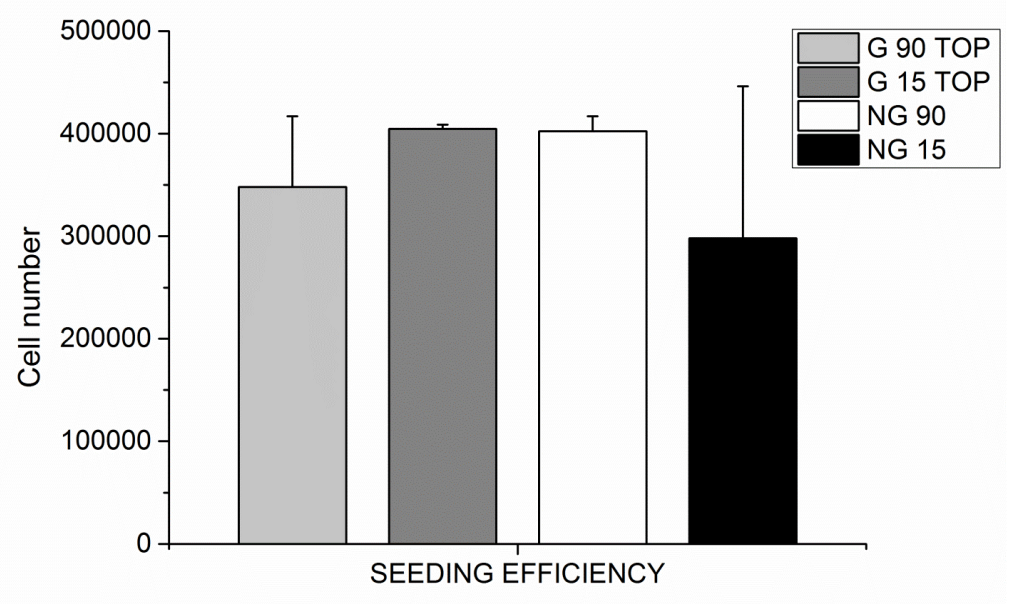

Figure S1. No major differences were seen between the samples after 8 hours from cell seeding and between the 2 gradient directions. When gradient scaffolds were seeded with the 0-90 region on top or with the 0-15 region on top, no difference in cell seeding efficiency was found, indicating that gravity didn't play a role with the cell culture method used. 

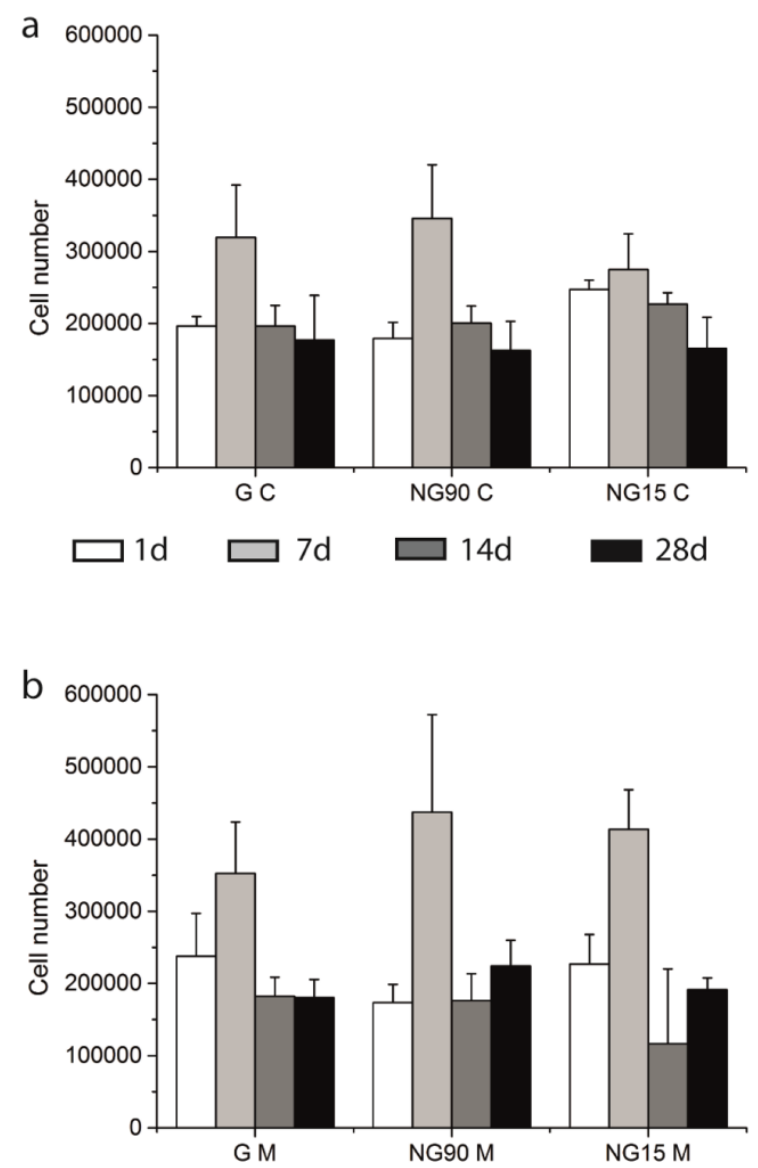

Figure S2. Cell number over time in the constructs cultured in chondrogenic (a) and osteogenic (b) medium. The same trend is shown by all the constructs over time, no major differences can be seen among the scaffolds at the same time point. 

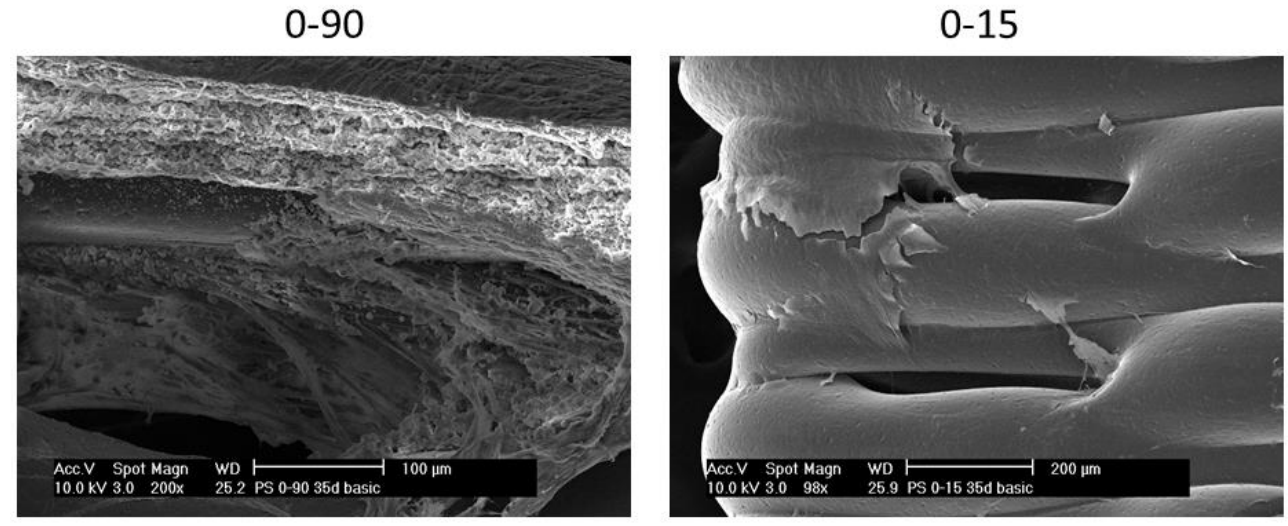

Figure S3. SEM images showing more rounded cells in the $0-90$ regions and more spread cells in the $0-15$ regions of the scaffolds.

\section{BMP-2}

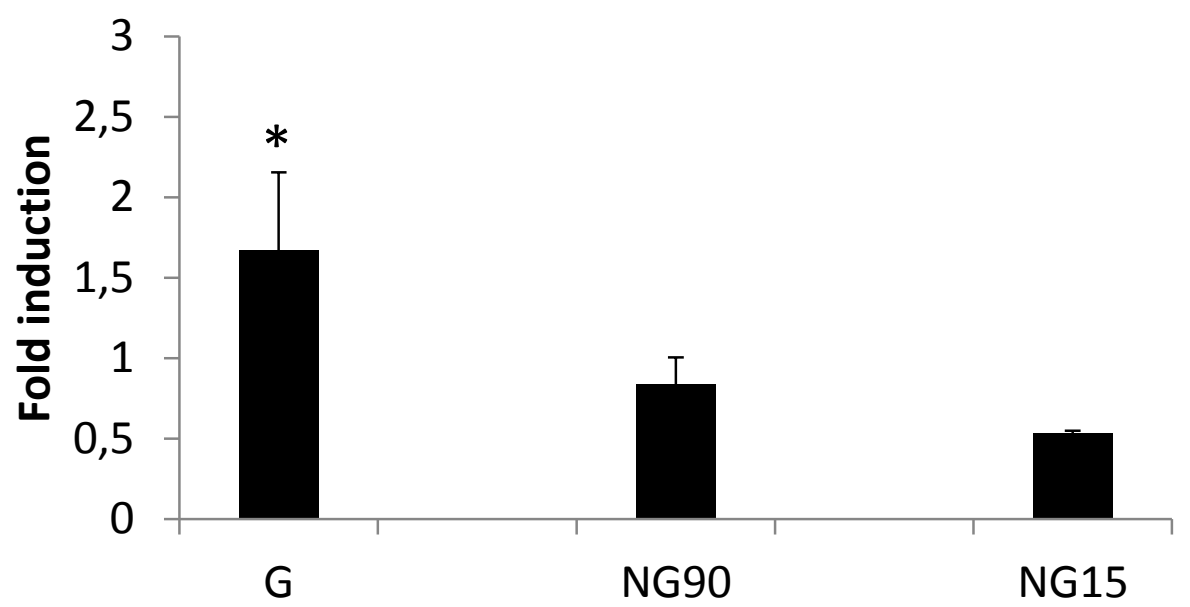

Figure S4. PCR analysis of BMP-2 gene expression in G, NG90, and NG15 scaffold after 2 weeks of culture in mineralization medium. Fold induction is expressed after normalization to NG90 scaffolds. ${ }^{*} p<0.05$. 

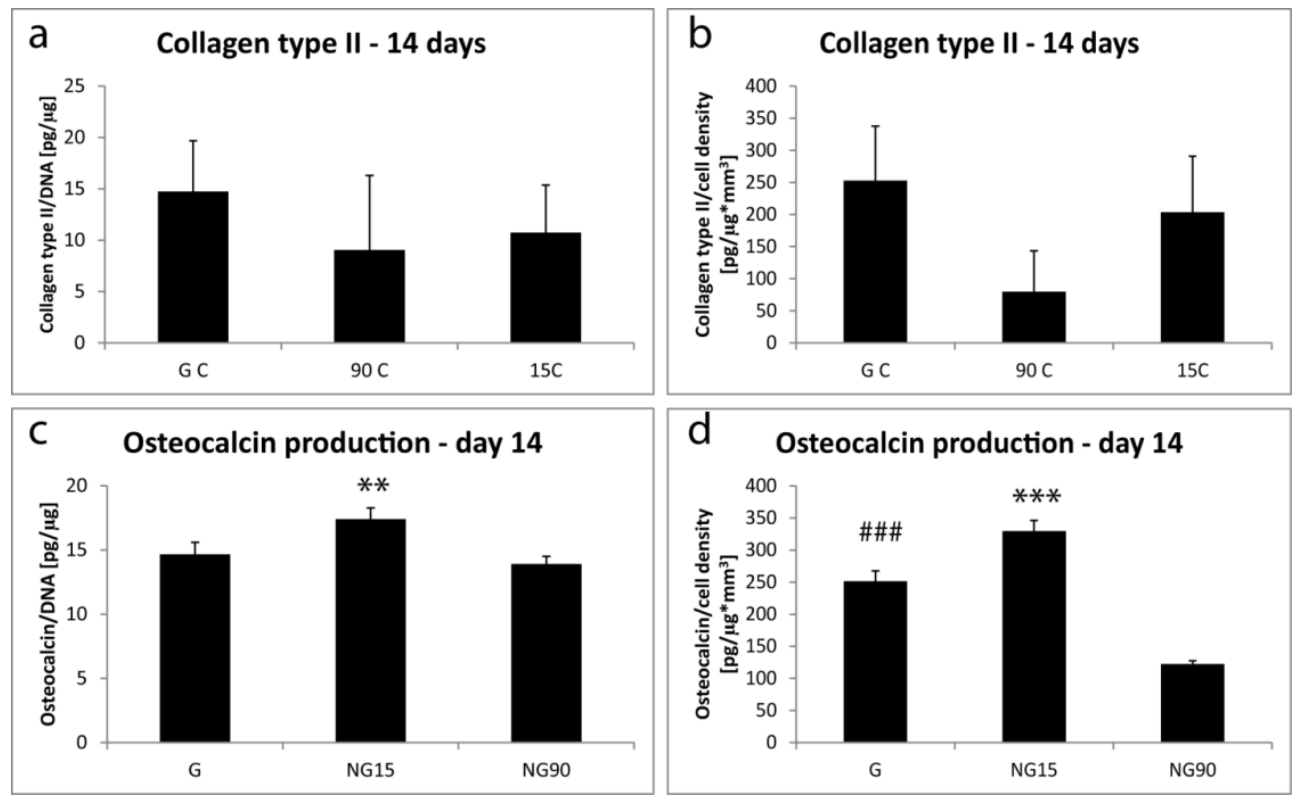

Figure S5. Collagen type II (A, B) and osteocalcin (C, D) production of hMSCs cultured in chondrogenic or osteogenic media after 2 weeks of culture. Protein production was normalized for DNA (A, C) as well as for cell density $(B, D)$ to take into account both different cell number and pore network volume in the gradient and non-gradient scaffolds. ${ }^{*} p<0.05$; ${ }^{* *} p<0,01 ;{ }^{* \star *}$ and \#\#\# $p<0.001$.
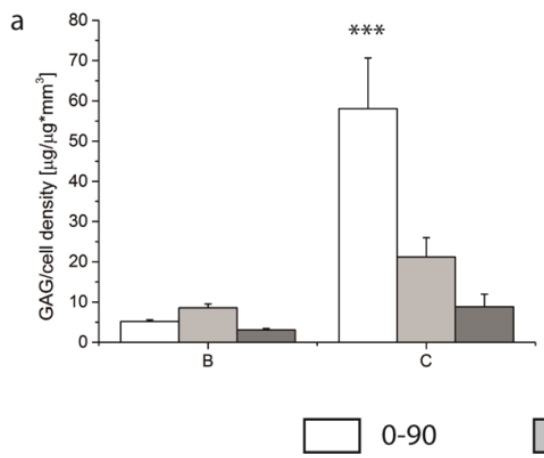

b

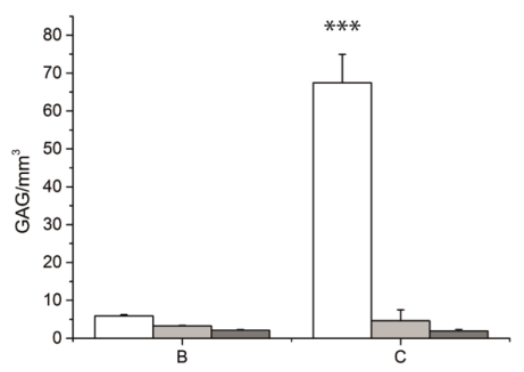

0-45

$0-30 / 0-15$

Figure S6. GAG amount in basic (B) and chondrogenic $(C)$ medium normalized by the cell density calculated as $\mu \mathrm{g}$ of DNA per pore volume (a) and normalized by pore volume only (b). The normalization didn't affect the trend no matter the conditions. ${ }^{\star \star \star}$ statistical significance with respect to the other conditions; $p<0.001$. 

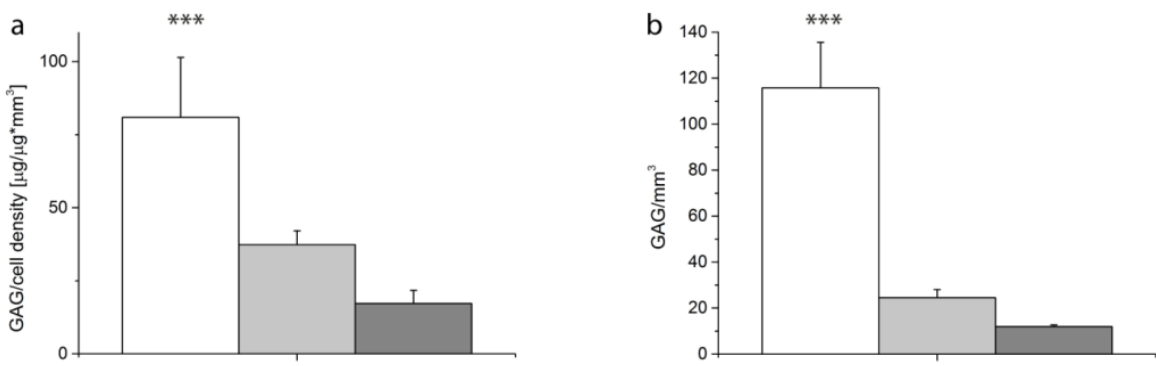

$0-90$

$0-45$

$0-30 / 0-15$

Figure S7. GAG amount in double medium normalized by the cell density calculated as $\mu \mathrm{g}$ of DNA per pore volume (a) and normalized by pore volume only (b). The normalization didn't affect the trend no matter the conditions. ${ }^{* * *}$ statistical significance with respect to the other conditions; $p<0.001$.
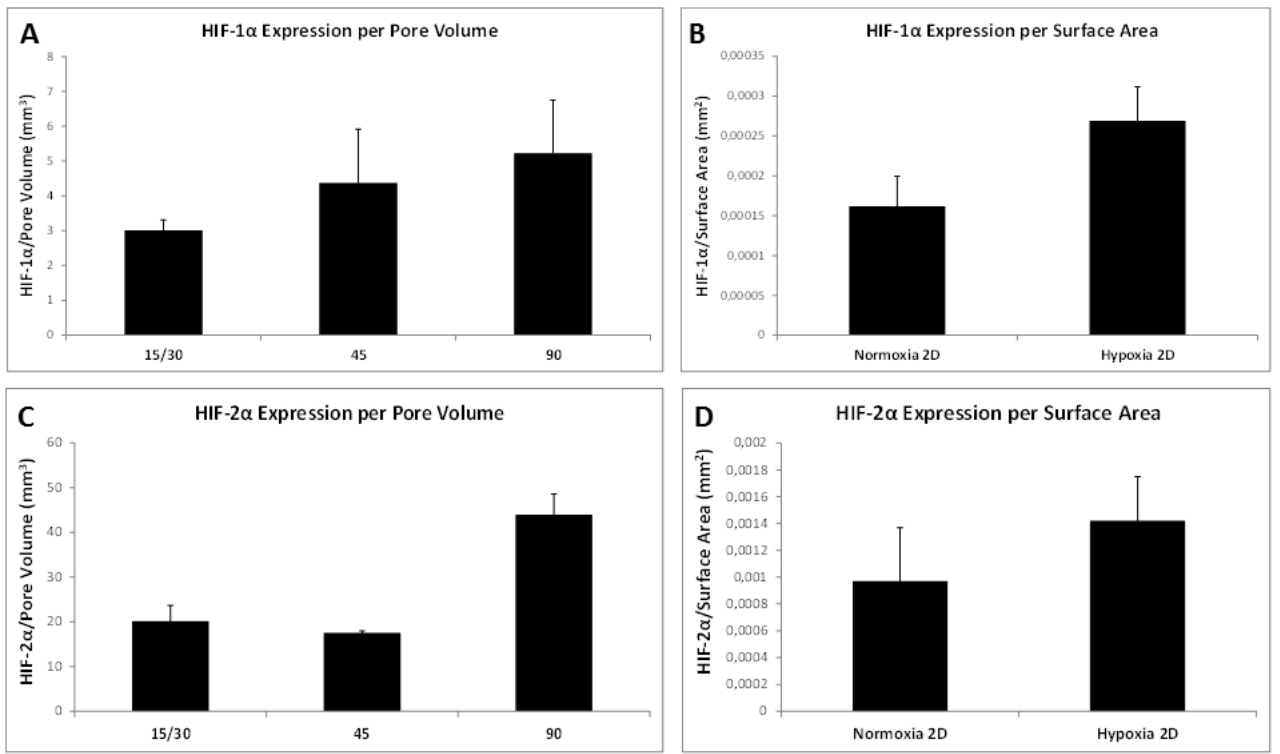

Figure S8. HIF-1 $\alpha(A, B)$ and HIF-2 $\alpha(C, D)$ protein expression in the different regions of the pore shape gradient scaffolds $(A, C)$ as well as in $2 D$ culture conditions $(B, D)$. Scaffold cultures were performed in basic media, while $2 \mathrm{D}$ cultures were performed both in normoxia as hypoxia conditions as controls. 
CHAPTER 6

\section{Surface energy and stiffness discrete gradients in additive manufactured scaffolds for osteochondral regeneration}

Andrea Di Luca, Alessia Longoni, Giuseppe Criscenti, Ivan Lorenzo-Moldero, Michel Klein-Gunnewiek, Julius Vancso, Clemens van Blitterswijk, Carlos Mota, Lorenzo Moroni

Published on Biofabrication. 2016 Feb 27;8(1):015014. 


\section{Abstract}

Swift progress in biofabrication technologies has enabled unprecedented progress in the application of developmental biology design criteria in three-dimensional scaffolds for regenerative medicine. Considering that tissues and organs in the human body develop following specific physico-chemical gradients, we hypothesized in this study that additive manufacturing technologies would significantly aid in the constructions of 3D scaffolds encompassing such gradients. Specifically, we considered surface energy and stiffness gradients and analyzed their effect on adult bone marrow derived mesenchymal stem cell differentiation into skeletal lineages. Discrete step-wise macroscopic gradients were obtained by sequentially depositing different biodegradable biomaterials in the additive manufacturing process, namely poly(lactic acid) (PLA), polycaprolactone (PCL), and poly(ethylene oxide terephthalate)/poly(butylene terephthalate) (PEOT/PBT) copolymers. At the bulk level, PEOT/PBT homogeneous scaffolds supported a higher alkaline phosphatase (ALP) activity compared to PCL, PLA, and gradient scaffolds, respectively. All homogeneous biomaterial scaffolds supported also a significantly higher amount of glycosaminoglycans (GAGs) production compared to discrete gradient scaffolds. Interestingly, the analysis of the different material compartments revealed a specific contribution of PCL, PLA, and PEOT/PBT to surface energy gradients. Whereas PEOT/PBT regions were associated to significantly higher ALP activity, PLA regions correlated with significantly higher GAG production. These results show that cell activity could be influenced by the specific spatial distribution of different biomaterial chemistries in a 3D scaffold and that engineering surface energy discrete gradients could be considered as an appealing criterion to design scaffolds for osteochondral regeneration. 


\section{Introduction}

The osteochondral interface is the tissue allowing the transition from articular cartilage to the underneath trabecular bone. Gradients in structural and physicocemical properties such as stiffness, surface energy, and mineral content are important to determine a smooth transition (1-3) between skeletal tissues with great differences in structural and mechanical properties, resulting consequently in different functions. Nowadays, the most common pathological state of the osteochondral tissue is osteoarthritis (OA). Currently available treatments in the clinics to regenerate osteochondral tissues, such as reparative surgery, allografts (4), autografts (5) and the implantation/transplantation of autologous chondrocytes

(6) are not yet completely successful. Besides limitations like the formation of fibrocartilage and lack of donor supply, these treatments inflict further tissue damage before any therapeutic effect can be achieved (6-8). In the last couple of decades regenerative medicine has focused on possible alternatives to improve current therapies. In particular, the use of scaffolds to support and direct the growth of already differentiated cells or precursor cell, such as human mesenchymal stem or stromal cells (hMSCs), has gained a lot of interest. When successful, in fact, this strategy could lead to the implantation of instructive scaffolds only, thus limiting patient discomfort of multiple surgeries and healthcare costs. To serve this objective, scaffolds need to match functional requirements such as allowing nutrient in-flow and metabolites out-flow, facilitating cell migration and ECM formation, provide sufficient mechanical properties and adequate surface properties to orchestrate cell activity.

Different materials can be used for the production of scaffolds. In osteochondral tissue regeneration, polymers such as poly-lactic-acid $(\operatorname{PLA})(9,10)$, polycaprolactone (PCL) (11-13) and poly(ethylene oxide terephthalate)/ poly(butylene terephthalate) PEOT/PBT copolymers $(14,15)$ seem promising biomaterial candidates owing to their physico-chemical properties apted to skeletal applications. Furthermore, these polymers have been already approved from regulatory bodies for targeted applications in the orthopedic field, thus offering a more facile route towards clinical translation. Among scaffold production 
techniques, several have been applied for osteochondral regeneration such as gas foaming (16), salt leaching (17) and freeze-drying (18). Yet, these techniques generate constructs that lack fundamental features such as pore interconnectivity, tailored and controlled pore size and shape, and resulting mechanical properties. In the past decade, additive manufacturing (AM) emerged as a powerful technology for scaffold design and fabrication, due to its ability to overcome these limitations. Via computer assisted design (CAD), AM allows to generate scaffolds with the desired shape, which is then converted in a program that is read by computer assisted manufacturing (CAM) software. The resulting scaffolds have a fully interconnected pore structure and features such as pore size, shape and fiber diameter can be varied during the entire manufacture process.

In recent years, hMSCs have found momentum in the proposed therapies for osteochondral regeneration. From a developmental point of view, both bone and cartilage originate from stem cells (19-21). Several studies proved the ability of hMSCs to differentiate toward, among others, the osteogenic and chondrogenic lineage in vitro (22-25). Their differentiation can be driven by different environmental cues such as nutrient and oxygen availability (26), surface and pore geometry $(27,28)$, presence of soluble factors (29-34) or substrate physicochemical properties. In this respect, a number of studies demonstrated that the interaction between cells and substrates determines changes in the cytoskeleton which may trigger differentiation processes $(27,35,36)$. Among physico-chemical properties, the surface energy of a biomaterial substrate determines the amount of adsorbed protein (37) and, consequently, the anchor points that cells will encounter at the moment of attachment, eventually determining their final shape and spreading degree. Cell shape is known to regulate cell fate: hMSCs with rounded shape tends to differentiate toward the chondrogenic lineage (38) whereas a spread shape leads to an osteogenic differentiation (39). Another cue that may trigger hMSC differentiation via a cytoskeleton modification is the substrate stiffness, in a process known as mechanotransduction (40,41). Cells cultured on a stiffer substrate will display a molecular expression pattern that leads to osteogenic differentiation (40), whereas cells grown on a softer substrate lead to differentiation toward a softer tissue lineage, such as chondrogenic differentiation (42). 
Despite a number of studies showing the influence of surface energy and stiffness in two-dimensional substrates, limited work has been performed in threedimensional (3D) scaffolds $(43,44)$. Here, we present a multi-material 3D scaffold fabricated by $\mathrm{AM}$ and based on three biodegradable polymers arranged in order to present a macroscopic step-wise discrete gradient either in surface energy or in stiffness. The influence of such 3D gradients on hMSC differentiation toward the osteogenic and chondrogenic lineages was evaluated as potential scaffold design criteria to aid in steering stem cell activity for osteochondral tissue regeneration.

\section{Materials \& Methods}

\subsection{Scaffolds preparation}

Scaffolds were fabricated via AM with a Bioscaffolder (SysENG, Germany). Scaffolds made of PEOT/PBT, PCL, and PLA alone (used as controls) or in combination in the same scaffold construct were produced. PEOT/PBT copolymers belong to the group of block co-polymers characterized by an aPEOTbPBTc nomenclature, where $a$ is the molecular weight of the starting PEG block and $b$ and $c$ are the PEOT/PBT ratio. Briefly, the polymers were placed in a stainless syringe and processed at $190{ }^{\circ} \mathrm{C}$ (300PEOT55PBT45, PolyVation, The Netherlands), 100 ${ }^{\circ} \mathrm{C}$ (PCL, Sigma-Aldrich, USA), or $210^{\circ} \mathrm{C}$ (PLA, PLAtimer 228, PolyVation, The Netherlands). The molten polymers were extruded through a cartridge unit, by the application of a nitrogen flow with a pressure of 5 bars from a pressurized cap and an extrusion screw rotation of $200 \mathrm{rpm}$. During plotting, the needle diameter and layer thickness were kept constant at $250 \mu \mathrm{m}, 200 \mu \mathrm{m}$ and $180 \mathrm{~mm} / \mathrm{min}$, respectively. The fiber spacing was kept constant at $750 \mu \mathrm{m}$. The translation speed of the printing arm was $180 \mathrm{~mm} / \mathrm{min}$ for PLA, $250 \mathrm{~mm} / \mathrm{min}$ for PCL, and 550 $\mathrm{mm} / \mathrm{min}$ for 300PEOT55PBT45. The plotted and tested samples were $6 \times 6 \times 3 \mathrm{~mm}$ blocks. The plotting process of the gradient scaffolds was based on the filling of 3 syringes, each with one polymer, heated to the right temperature. The CAD/CAM program was written for the manufacturing of 6 layers of each polymer and then 
change the syringe. The sequence of the surface energy (wettability) gradient was PLA-300PEOT45/PBT55-PCL (WG), while the stiffness gradient followed the order PLA-PCL-300PEOT45/PBT55 (SG).

\subsection{Polymer films and contact angle measurements}

In order to determine the surface energy, static contact angle measurements with water were performed by the sessile drop technique using an optical contact angle device equipped with an electronic syringe unit (OCA15, Dataphysics, Germany). To have a flat surface and to maintain the same temperature treatment as in AM processing, a film of each polymer was made via hot embossing. We reasoned that, being wettability a surface property related to how the material is processed, contact angle measurements on such hot embossed films could be equivalently representative of contact angle values of 3D scaffolds. Contact angle measurements on $3 \mathrm{D}$ scaffolds were otherwise challenging to test, due to the fact that water droplets were immediately entrapped in the scaffolds' pore network due to capillary forces. Specifically, PCL, 300PEOT55PBT45 and PLA discs of $500 \mu \mathrm{m}$ thickness were made by a hot-embossed compression moulding technique. Granules of each polymer were distributed inside circular punched moulds of stainless steel and placed between two silicon wafers functionalized with $1 \mathrm{H}, 1 \mathrm{H}, 2 \mathrm{H}, 2 \mathrm{H}$-perfluorodecyltrichorosilane (FDTS, Sigma-Aldrich). The wafermould-wafer stack was placed in the aperture of the temperature hydraulic press (Fortune Holland) at $80^{\circ} \mathrm{C}$ for PCL, $180^{\circ} \mathrm{C}$ for $300 \mathrm{PEOT55PBT} 45$ and $230^{\circ} \mathrm{C}$ for PLA and 10 bar. After 5 minutes the system was cooled to $60^{\circ} \mathrm{C}$ and the pressure was released. The mould and wafer were manually separated to provide smooth discs. The discs were used for contact angle analysis, as above described.

\subsection{Mechanical testing at the macroscopic length scale}

The mechanical properties of the gradient scaffolds and the controls in wet and dry conditions were evaluated by uniaxial compression tests. For each configuration, 6 samples were analyzed. Samples were positioned in a standard compression block and aligned to the $500 \mathrm{~N}$ load cell of a Zwick materials-testing machine. To 
evaluate the influence of an aqueous environment and possible perfusion effects, tests were performed under two different environmental conditions: in a nitrogen atmosphere (dry condition) and in culture medium (alpha-MEM) at $37^{\circ} \mathrm{C}$ (wet condition). In the second case, the samples were left overnight in the medium at 37 ${ }^{\circ} \mathrm{C}$ and a modified compression block was used to perform the test. All specimens were preloaded with $0.1 \mathrm{~N}$ and preconditioned by a series of ten cycles until a strain of $5 \%$, with a strain rate of $1 \mathrm{~mm} / \mathrm{min}$ to reduce the hysteresis. Subsequently, they were compressed at $1 \mathrm{~mm} / \mathrm{min}$ until failure. A stress-strain curve representing the mechanical properties of the samples can be obtained from the cross-sectional area and the strain measurements. From the stress-strain curves, the following parameters were obtained: Young's modulus $(\mathrm{Pa})$, defined as the slope of the linear region of the stress-strain curve, ultimate stress $(\mathrm{Pa})$, ultimate strain (\%) and strain energy density $(\mathrm{Pa})$ at failure.

\subsection{Mechanical testing at the microscopic length scale}

Force measurements were performed on 3D scaffolds in air using a Dimension 3100 AFM equipped with a hybrid scanner and a NanoScope IVa controller (Veeco/Digital Instruments (DI), Santa Barbara, CA, USA). Commercially available silicon cantilevers (PointProbe ${ }^{\circledR}$ Plus silicon probes, PPP-NCH, Nanosensors, Neuchatel, Switzerland) with a spring constant of around $42 \mathrm{Nm}^{-1}$ were used in the experiments. Force measurements were carried out with a z-ramp size of $2 \mu \mathrm{m}$, a scan rate of $1 \mathrm{~Hz}$, and a deflection trigger of $200 \mathrm{~nm}$. For every material around 1000 force curves were taken $(n=1000)$. The stiffness of the materials was defined as the slope of the approach curve from the point of contact up to an indentation force of $1 \mu \mathrm{N}$.

\subsection{Cell expansion and culture}

Pre-selected hMSCs (male, age 22) were retrieved from the Institute of Regenerative Medicine (Temple, Texas). Briefly, a bone marrow aspirate was drawn and mononuclear cells were separated using density centrifugation. The cells were plated to obtain adherent hMSCs, which were harvested when cells 
reached $60-80 \%$ confluence. These were considered passage 0 (P0) cells. These P0 cells were expanded, harvested and frozen at passage 1 (P1) for distribution. Cells were grown in MSC proliferation medium, which contains minimal essential medium ( $\alpha$-MEM, Gibco, Breda, The Netherlands) supplemented with 10\% fetal bovine serum (FBS, Lonza), $100 \mathrm{U} / \mathrm{ml}$ penicillin (Gibco, Breda, The Netherlands), $10 \mu \mathrm{g} / \mathrm{ml}$ streptomycin (Gibco, Breda, the Netherlands), $2 \mathrm{mM}$ L-glutamine (Gibco, Breda the Netherlands), $0.2 \mathrm{mM} \mathrm{L}$-ascorbic acid 2-phosphate magnesium salt (ASAp, Sigma-Aldrich, Zwijndrecht, The Netherlands) and $1 \mathrm{ng} / \mathrm{ml}$ of basic fibroblast growth factor-2 (bFGF-2, Fisher Scientific, Landsmeer, the Netherlands) at $37{ }^{\circ} \mathrm{C}$ in a humid atmosphere with $5 \% \mathrm{CO}_{2}$. Cells were expanded up to approximately $80 \%$ confluency and either frozen for further use or seeded on the scaffolds.

\subsection{Cell seeding on scaffolds}

Briefly, after trypsinizaition with $0.25 \%$ trypsin (Life Technologies, Bleiswijk, the Netherlands), cells (passage 2-4) were counted using a Bückner chamber and resuspended in proliferation media at a density of 500'000 cells in $40 \mu \mathrm{L}$.

The day before seeding, scaffolds were disinfected in $70 \% \mathrm{EtOH}$ for 30 min under stirring, washed 3 times in phosphate buffered saline solution (PBS, Lonza, Breda, the Netherlands), and incubated overnight in cell proliferation media to allow protein adsorption on the scaffold's fibers. After protein adsorption, the $40 \mu \mathrm{L}$ of cell suspension were placed on the scaffold in a drop wise fashion to account for a cell seeding density of 500'000 cells/scaffold. The seeded scaffolds were placed for 4 hours in the incubator to allow cell adhesion before adding the cell culture media.

Cells were cultured on the gradient $(G)$ and non-gradient (NG) scaffolds for 7 days in proliferation media. At day 7 , the proliferation media was changed and the cells within the scaffolds were cultured for another 7 and 28 days in basic media, mineralization media consisting of basic media supplemented with $10 \mathrm{nM}$ dexamethasone (Sigma-Aldrich, Zwijndrecht, the Netherlands) and $10 \mathrm{mM} \beta$ glycerol-phosphate (Sigma-Aldrich, Zwijndrecht, the Netherlands), and chondrogenic media consisting of DMEM supplemented with $50 \mathrm{mg} / \mathrm{mL}$ ITS-premix 
(Bexton Dickinson), $0.4 \mathrm{mM}$ Proline (Sigma-Aldrich, Zwijndrecht, the Netherlands), $50 \mathrm{mg} / \mathrm{mL}$ ascorbic acid (ASAp, Sigma-Aldrich, Zwijndrecht, the Netherlands), 100 $\mathrm{mg} / \mathrm{mL}$ sodium pyruvate (Sigma-Aldrich, Zwijndrecht, the Netherlands), $100 \mathrm{U} / \mathrm{ml}$ penicillin (Life Technologies, Bleiswijk, the Netherlands), $10 \mu \mathrm{g} / \mathrm{ml}$ streptomycin (Life Technologies, Bleiswijk, the Netherlands), $10 \mathrm{ng} / \mathrm{mL}$ transforming growth factor $\beta 3$ (TGF- $\beta 3$ ) (Life Technologies, Bleiswijk, the Netherlands) and $10^{-7} \mathrm{M}$ dexamethasone (Sigma-Aldrich, Zwijndrecht, the Netherlands).

\subsection{Biochemical study}

\subsubsection{DNA analysis}

The cell number per scaffold was calculated from the $\mu \mathrm{g}$ of DNA, obtained by a Cyquant DNA assay kit (Life Technologies, Bleiswijk, the Netherlands). Briefly, each scaffold was cut to improve lysis efficiency and freeze-thawed 5 times. After the freeze-thawing process, cells within the scaffolds were lysated by diluting the 20x lysis buffer provided with the kit using a saline buffer $(180 \mathrm{mM} \mathrm{NaCl}, 1 \mathrm{mM}$ EDTA in distilled water). After $1 \mathrm{~h}$ of lysis, samples were sonicated 2 times for 10 seconds using a Branson sonifier 250 (Emerson Industrial Automation, USA). DNA content was quantified with a CyQuant kit (Invitrogen, Breda, the Netherlands) according to manufacturer's protocol and fluorescence was measured at $480 \mathrm{~nm}$ using a spectrophotometer LS50B (Perkin Elmer, The Netherlands). DNA concentrations were calculated from a $\lambda$ DNA standard curve.

\subsubsection{ALP activity}

To evaluate hMSC differentiation toward the osteogenic lineage, ALP content was measured using a CDP star kit (Roche, Woerden, the Netherlands). For this purpose, $10 \mu \mathrm{L}$ of sample was added to a well of a white 96 -well plate and $40 \mu \mathrm{L}$ of substrate (Disodium 2-chloro-5-(4-methoxyspiro [1,2-dioxetane-3,2'-(5'chloro)tricycle[3.3.1.13.7]decan]-4-yl)-1-phenyl phosphate) was added. After 15 
minutes incubation, luminescence was read using a spectrophotometer LS50B (Perkin Elmer). ALP activity was corrected for DNA content.

\subsubsection{GAG amount}

To evaluate the differentiation toward the chondrogenic lineage, glycosaminoglycans (GAG) amount was quantified using 1,9-Dimethyl Methylene Blue (DMMB) assay. Specifically, $25 \mu \mathrm{L}$ of sample were placed into a transparent flat bottom 96 well plate and $5 \mu \mathrm{L}$ of $2.3 \mathrm{M} \mathrm{NaCl}$ solution were added. Then, $150 \mu \mathrm{L}$ of DMMB solution were added and absorbance was read using a Multiscan Go (Thermo Scientific, USA) plate reader at a wavelength of $525 \mathrm{~nm}$. GAG content was quantified with a chondroitin sulphate standard curve and corrected for DNA content.

\subsection{Microscopy analysis - optical stereo and SEM}

$G$ and NG scaffolds were analyzed by optical stereo microscopy and scanning electron microscopy (SEM, Philips - XL 30 ESEM-FEG). Directly after plotting, scaffolds were cut in half and pictures were taken with a stereological microscope. Afterwards, the samples were gold sputtered and analyzed by SEM. Scaffolds cultured in mineralization media for 3,7 and 28 days were fixed using $10 \%$ formalin, dehydrated by an increased series of ethanol concentration (50-60-70-80$90-96-100 \%)$ and cut in half. The final dehydration step was carried out via immersion in hexamethyldisilazane (Sigma Aldrich) and overnight evaporation. Dry scaffolds were mounted on SEM stubs, gold sputtered (Cressington sputter coater 108 auto), and analyzed using $10 \mathrm{kV}$ and a working distance of $25 \mathrm{~mm}$.

\subsection{Statistical analysis}

All quantitative data are expressed as mean \pm standard deviation (SD). Statistics were performed using IBM SPSS Statistics 20. A two-way statistical analysis of variance (ANOVA) with a significant $p$ level of 0.05 was used to determine differences between the groups and test conditions. Tukey's multiple comparisons test was used to perform post hoc analysis. Statistical significance 
between the control group and the experimental ones is indicated with $\left(^{*}\right)$ which represents a $p$-value $<0.05,\left({ }^{* *}\right)$ which represents a $p$-value $<0.01$, and $\left({ }^{* * *}\right)$ which represents a $p$-value $<0.001$.

\section{Results}

\subsection{Discrete gradient generation - contact angle measurements}

To determine the surface energy gradient, the contact angle of the single material was measured on polymer films. PLA showed a contact angle of $66.5^{\circ} \pm 3.1^{\circ}$, followed by PEOT/PBT $68.4^{\circ} \pm 9.4^{\circ}$ and PCL with the highest value $77.6^{\circ} \pm 8.6^{\circ}$. The chosen polymer sequence for the surface energy discrete gradient was PLAPEOT/PBT-PCL (Figure 1a).

a

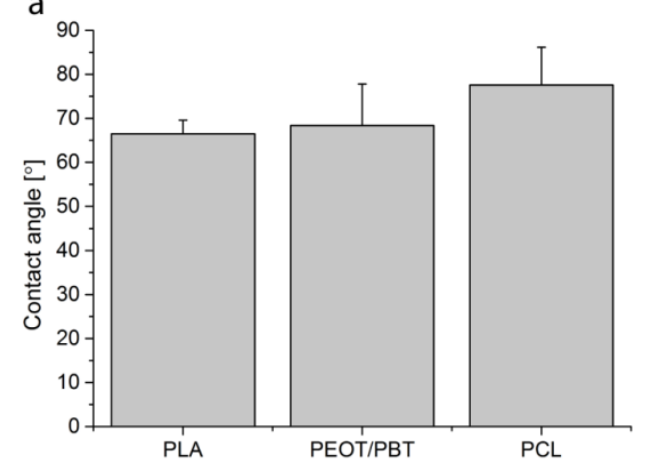

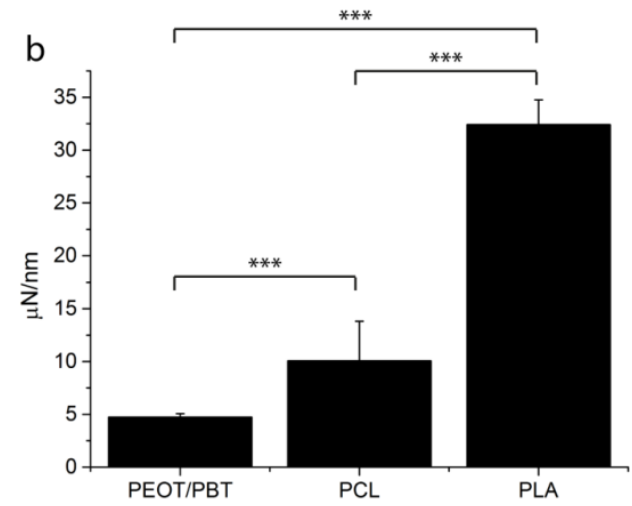

Figure 1. Graph showing the contact angle of the different polymers (a) and the stiffness measured in the middle of one fiber of the scaffold per polymer (b). The contact angle increases along the discrete gradient from PLA to 300PEOT55PBT45 and PCL. The stiffness gradient showed a sequence from 300PEOT55PBT45 to PCL, and PLA. 

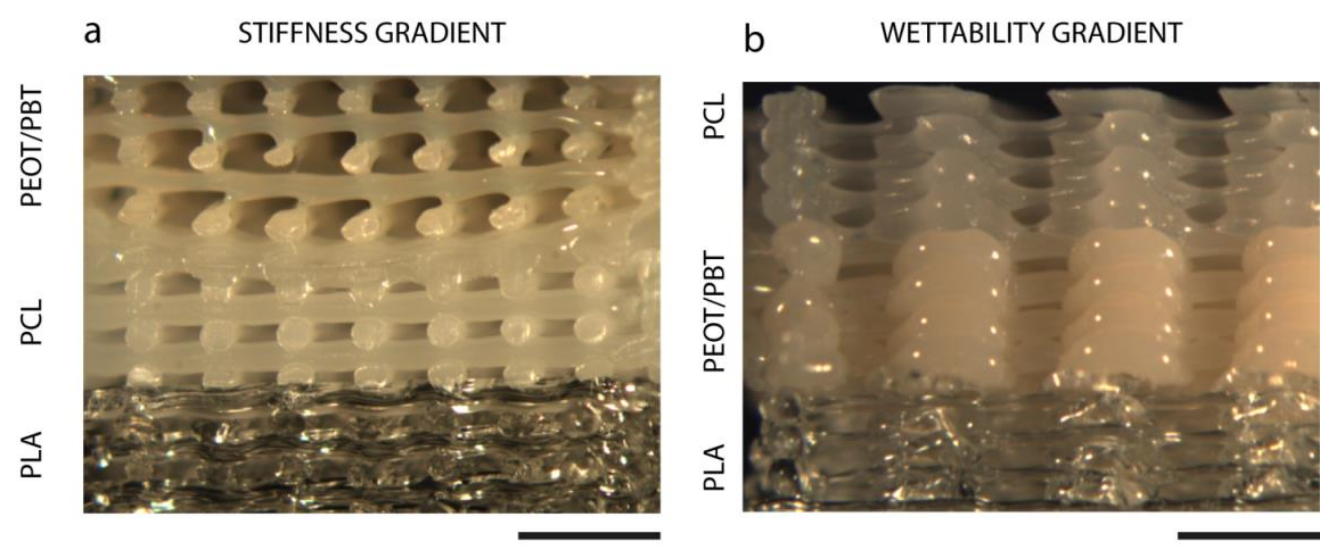

Figure 2. Steromicroscopical pictures of the cross section of the stiffness gradient scaffolds (a) and the wettability gradient scaffolds (b). Scale bar: $2 \mathrm{~cm}$.

\subsection{Gradient generation - stiffness}

The local surface mechanical properties of the 3 different polymers used to fabricate discrete gradient scaffolds were measured by AFM over 1000 different locations per scaffold region (Figure 1b). PEOT/PBT displayed a stiffness of $4.73 \pm$ $0.35 \mu \mathrm{N} / \mathrm{nm}, \mathrm{PCL}$ of $10.07 \pm 3.74 \mu \mathrm{N} / \mathrm{nm}$, and PLA of $32.41 \pm 2.34 \mu \mathrm{N} / \mathrm{nm}$. The chosen sequence following the stiffness gradient was, therefore, PEOT/PBT-PCLPLA (Figure 2).

The mechanical properties of the discrete gradient scaffolds and relative homogenous scaffold controls in wet and dry conditions were evaluated to further characterize the fabricated constructs at a structural level. Comparing the Young's modulus in dry conditions, the three materials showed a different mechanical behaviour (Figure 3). PEOT/PBT showed the lowest value $(17.07 \pm 0.99 \mathrm{MPa})$, followed by PCL $(50.62 \pm 1.38 \mathrm{MPa})$ and PLA $(107.86 \pm 9.8 \mathrm{MPa})$, respectively. The discrete gradient scaffolds displayed in dry and wet conditions a Young's modulus close to the most compliant PEOT/PBT scaffolds (Figure 3). In addition, the WG scaffolds showed a higher ultimate stress both in dry and wet conditions compared to SG ones. However, an opposite trend was detected for the ultimate strain in dry and wet conditions. Finally, the SG scaffolds showed a higher strain energy density both in dry and wet conditions compared to the WG ones. 

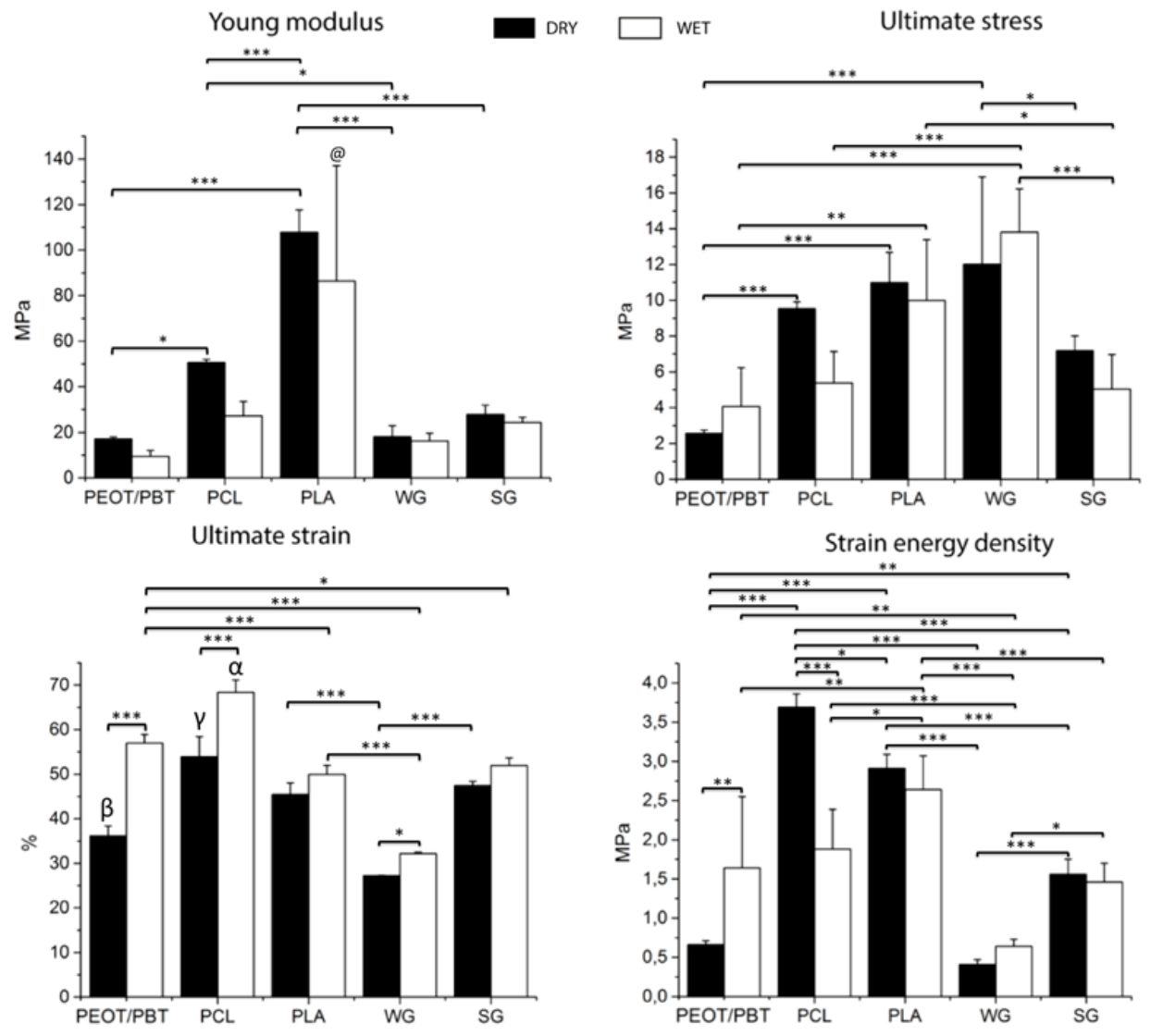

Figure 3. Mechanical analysis of single material and gradient scaffolds. Results are expressed as plot showing the Young's modulus, the ultimate stress, ultimate strain, and strain energy density. @ means that PLA in wet condition is statistically different ( $p$-value < 0.001 ) compared to the other materials in the same conditions. a means that PCL in wet condition is statistically different $(p$-value $<0.001)$ compared to the other materials in the same condition. $\beta$ means that PA in dry condition is statistically different ( $p$-value $<0.001$ ) compared to the other materials in the same condition. $\mathrm{Y}$ means that PCL in dry condition is statistically different $(p$-value < 0.001) compared to the other materials in the same condition.

\subsection{Cell seeding efficiency and cell shape}

Cell seeding efficiency was not affected by the material or the type of gradient (Supporting Information, Figure S1). All structures presented a cell seeding efficiency between 40 and $50 \%$, with a slight increase for the wettability gradient constructs. Cells displayed a different shape depending on the material they 
adhered on (Figure 4). After 3 days of culture, hMSCs appeared homogeneously distributed in all the samples, their shape was elongated in PCL and PEOT/PBT, while on PLA their morphology looked more spread.
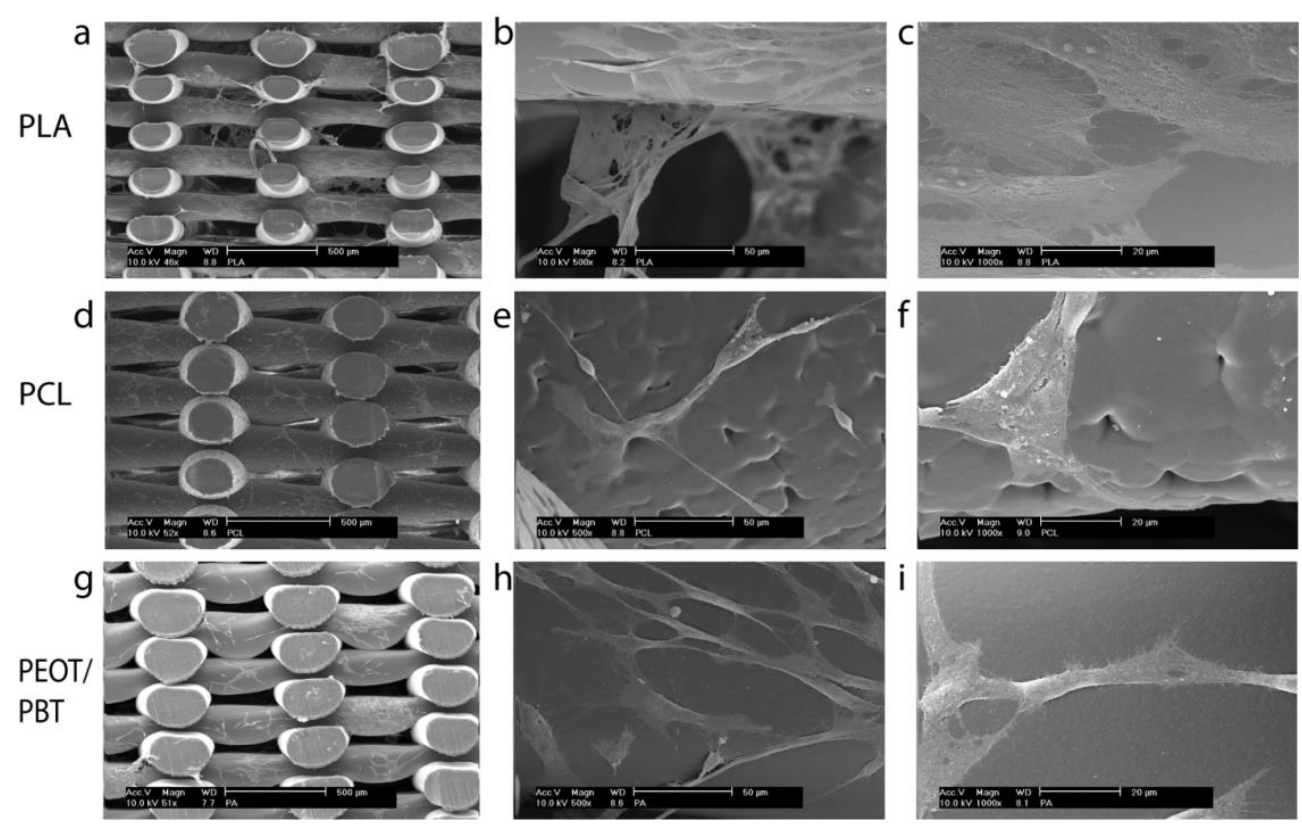

Figure 4. SEM micrograph depicting the cell shape on the materials. On PLA hMSCs present a spread phenotype on the fibers with part of them bridging two fibers $(a, b, c)$. On PCL (d, e, f) and PEOT/PBT (g, h, i) hMSCs showed an elongated shape. Scale bar $500 \mu \mathrm{m}$ $(\mathrm{a}, \mathrm{d}, \mathrm{g}), 50 \mu \mathrm{m}(\mathrm{b}, \mathrm{e}, \mathrm{h})$ and $20 \mu \mathrm{m}(\mathrm{c}, \mathrm{f}, \mathrm{i})$.

\subsection{Osteogenic and chondrogenic differentiation}

The ALP activity profile over time was the same for gradient and non-gradient scaffolds. ALP activity increased over time. After 28 days of culture in differentiation conditions, PEOT/PBT scaffolds outperformed the other constructs. When cultured under basic conditions, no major differences could be seen among the samples cultured for the same amount of time. Yet, an increase in ALP activity was observed after 28 days of culture in all the scaffolds compared to 7 days of culture.

Similarly, the GAG amount produced by the cells within the samples cultured in basic media did not show significant differences. When hMSCs were grown under 
basic conditions the single material constructs displayed a decreased (PCL, PLA) or a stable (PEOT/PBT) GAG amount. The GAG amount increased in the gradient scaffolds over time, yet not showing consistent differences with the single material scaffolds. Under chondrogenic conditions, the surface energy gradient (WG) outperformed the other constructs including the stiffness gradient at day 7. This beneficial effect was lost after 28 days, in which the GAG content of the single material constructs was significantly higher with respect to the gradient samples.
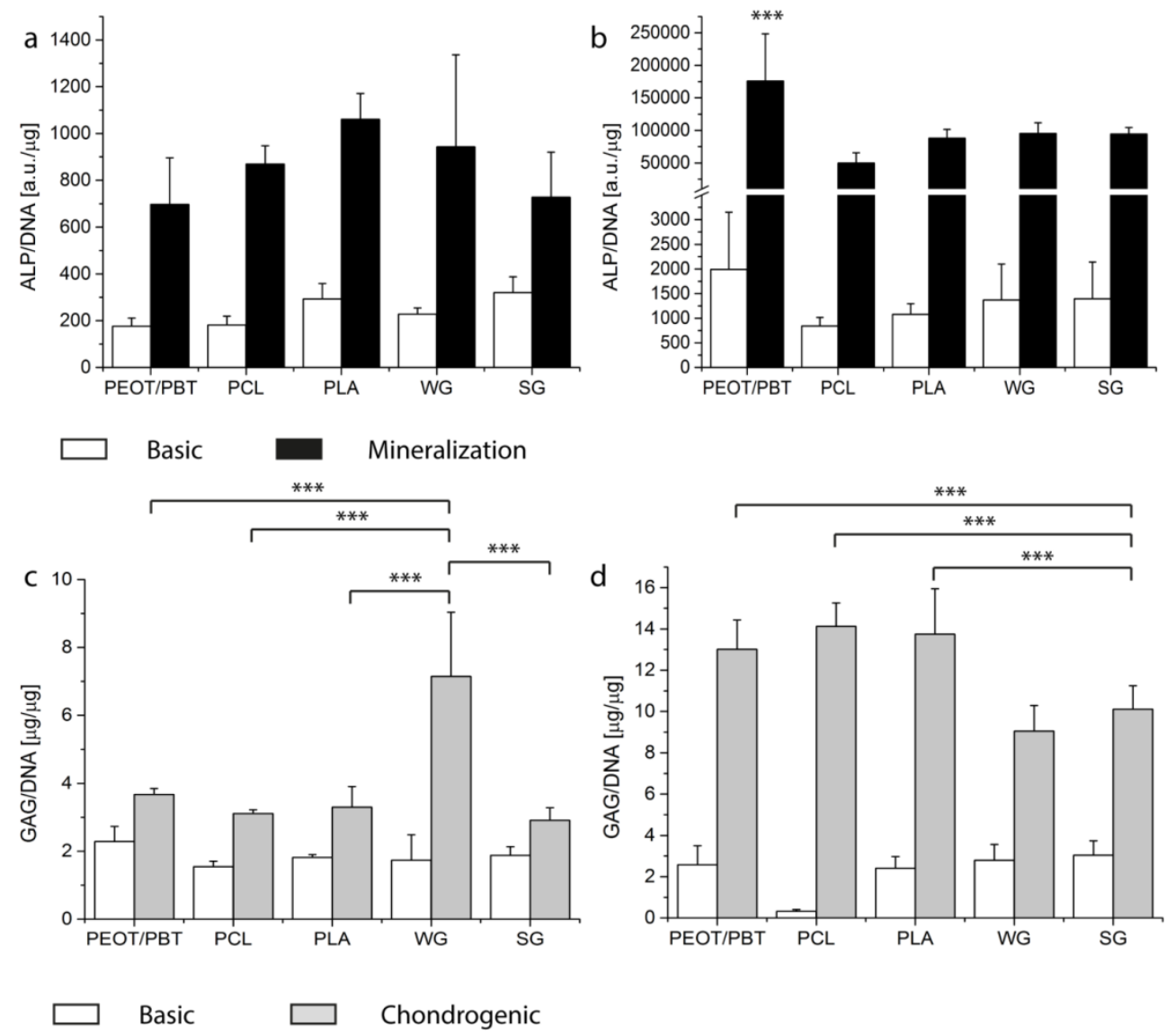

Figure 5. Graphs showing ALP activity (a, b) and GAG amount (c, d) in the full scaffolds at 7 $(a, c)$ and $28(b, d)$ days of culture. The ALP activity in all the samples was significantly increased over time. 300PEOT55PBT45 scaffolds showed the highest ALP activity after 28 days in differentiation media (b). At 7 days under chondrogenic conditions WG scaffolds showed the highest GAG content (c). After 28 days under chondrogenic conditions the single material scaffolds outperformed the gradient scaffolds in GAG content (d). ${ }^{* * *}$ statistical significance, $p<0.001$ 


\subsection{Osteogenic and chondrogenic differentiation partition analysis}

To study whether the presence of these specific biomaterials in different regions of the gradient scaffolds had a net influence on cell differentiation, the scaffolds were segmented in the different material regions and analysed. After 7 days under mineralization conditions cells residing in the 300PEOT55PBT45 zone of the stiffness gradient showed the highest ALP activity level (Figure 6) compared to the other regions. No other significant changes were, however, observed for the stiffness gradient constructs. Interestingly, in surface energy gradient scaffolds the 300PEOT55PBT45 region supported a significantly higher amount of produced GAGs compared the other material zones under chondrogenic conditions ay day 7 , whereas the PLA region outperformed the other materials at day 28 (Figure 7). When cultured in mineralization medium, cells adhering on the PEOT/PBT regions showed significantly higher levels of ALP activity with respect to the levels displayed on PCL and PLA compartments. 

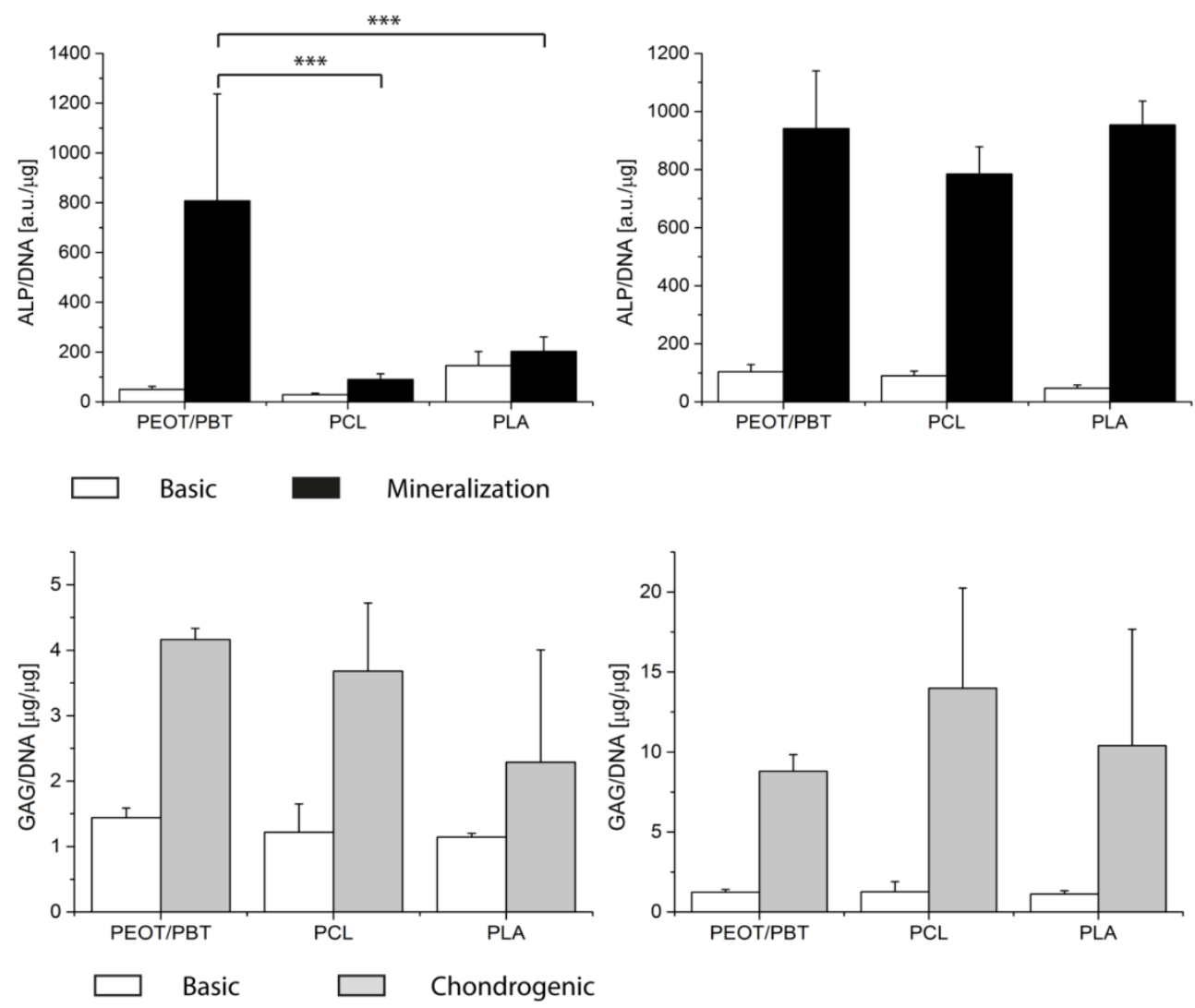

Figure 67. Plots showing the osteogenic and chondrogenic differentiation of hMSCs expressed as ALP activity (a, b) and GAG amount (c, d) at day 7 (a, c) and day 28 (b, d) per region of the stiffness gradients. At 7 days, the 300PEOT55PBT45 showed a significantly higher ALP activity in the stiffness gradient. In the wettability gradients no major differences could be depicted after 7 days, whereas after 28 days the 300PEOT55PBT45 region outperformed the other material regions. Under chondrogenic conditions no major differences could be seen in the stiffness gradient at 7 and 28 days. In the WG scaffolds 300PEOT55PBT45 showed a greater amount of GAG after 7 days. After 28 days PLA was the material with the highest GAG amount. 

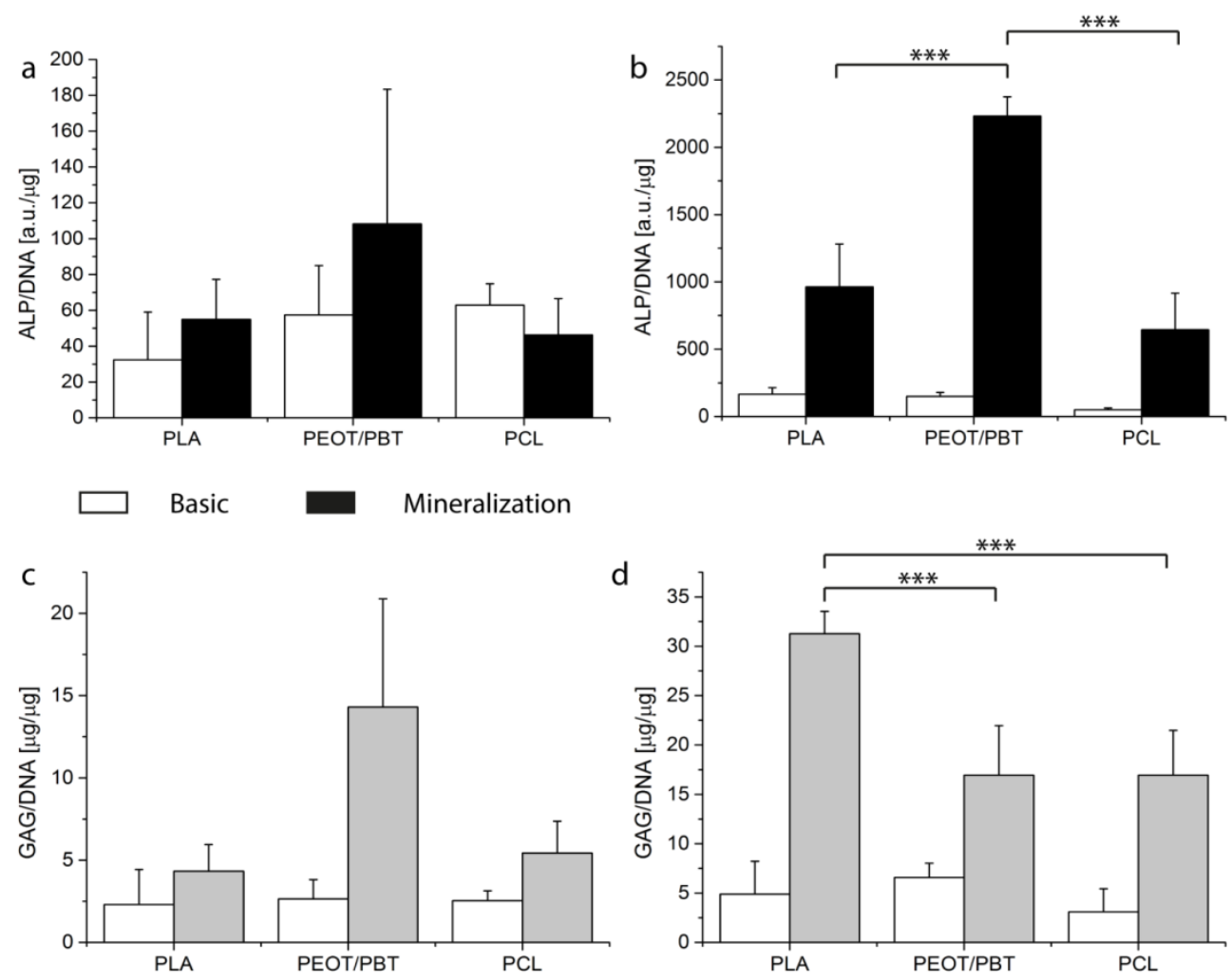

Basic $\square$ Chondrogenic

Figure 7. Graphs showing the osteogenic and chondrogenic differentiation of hMSCs expressed as ALP activity (a, b) and GAG amount (c, d) at day 7 (a, c) and day 28 (b, d) per region of the surface energy gradients. No major differences could be depicted after 7 days, whereas after 28 days the 300PEOT55PBT45 region outperformed the other material regions for ALP activity. Under chondrogenic conditions, PLA was the material with the highest GAG amount after 28 days of culture.

\section{Discussion}

Several physico-chemical cues provided by biomaterials may act on the differentiation pattern of hMSCs. Among these, surface chemistry and mechanical properties are known to play an important role in two-dimensional substrates (45, 46). In the present study PCL, PLA and 300PEOT55PBT45 were plotted within the same scaffold in order to obtain a discrete gradient in surface energy and stiffness. The chosen polymers are FDA approved and widely used in osteochondral tissue 
engineering. PCL and PLA scaffolds alone or in combination with biological signals and cells have already shown promising results in vivo for bone regeneration (47, 48), whereas 300PEOT55PBT45 was studied for cartilage regeneration, reaching clinical evaluation $(49,50)$. The design of the surface energy gradient scaffolds was based on the analysis of the biomaterials wettability via water contact angle. The three materials chosen showed a similar surface energy, though a trend was observed with an increasing contact angle spanning from PLA to 300PEOT55PBT45 and PCL (Figure 1a). In the design of the stiffness gradient scaffolds, differences in both bulk and surface mechanical properties were more pronounced and. While bulk mechanical properties were measured to characterize the structural mechanical behavior of the fabricated scaffolds, local surface mechanical properties were determined to decide the order of the 3 biomaterials in the final discrete gradient scaffolds. As expected, the local stiffness results revealed a greater stiffness of PLA followed by PCL and 300PEOT55PBT45. This was also confirmed at a scaffold macrostructural level by uniaxial compression, which becomes more relevant as the cells start to produce their ECM and occupy the pore volume.

The obtained mechanical properties adequately match the initial ones of the complex osteochondral structure $(51,52)$. The use of the same geometry and scaffold layers for the scaffold macrostructure allowed obtaining a similar cross sectional area among the three different regions that promoted a discrete gradient of structural properties. During uniaxial compression test, the trimaterial scaffold regions are subjected to the same stress. For this reason, in first approximation, the mechanical behavior of the trimaterial scaffolds could be homogenized using a Reuss model. In this case, the mechanical response is described by equation 1 :

$$
E_{\text {tot }}=\frac{3 \cdot E_{P E O T / P B T} \cdot E_{P C L} \cdot E_{P L A}}{E_{P E O T / P B T} \cdot E_{P C L}+E_{P C L} \cdot E_{P L A}+E_{P E O T / P B T} \cdot E_{P L A}}
$$

From equation 1, it is possible to conclude that the total Young's modulus is strongly dependent from the PEOT/PBT region that has the lowest Young's modulus. 
In 2005, Yeung et al. were among the first to show the correlation between substrate stiffness and cell shape (53). The stiffness of the substrate is known to determine cytoskeleton modifications, in particular on the stress fibers. This modification results in a change of cell shape, which for stem cells is associated to a variation of their activity (54). Cells on stiffer substrates showed a more spread phenotype compared to cells adhering on softer ones. In this study hMSCs showed spread morphology on all biomaterials, which might also explain the apparent lack of influence of the biomaterial stiffness in the evaluated range. Cells adhering on stiffer PLA showed a flatten morphology, which appeared more spindle-like on 300PEOT55PBT45 and on PCL (Figure 4). The adsorption onto the material surface of serum and ECM proteins plays also an important role in cell adhesion and consequently cell morphology (55). Protein adsorption is influenced by the material surface properties such as surface charge and chemistry, ultimately resulting in a different wettability. Tamada et al. studied protein adsorption onto several polymeric surfaces and found that the maximum amount of protein adsorbed on culture substrates was on surfaces with water contact angles ranging between $60^{\circ}$ and $80^{\circ}(56)$. The materials used in this study presented a contact angle in the range identified by Tamada (Figure 1a). The contact angle was optimal for cell adhesion whereas the differences in surface stiffness determined a variation in cell morphology on the materials composing the discrete gradient scaffolds.

Although gradient scaffolds did not show any beneficial effect in the differentiation of hMSCs in absence of differentiation soluble factors over a culture period of 4 weeks, an effect of the different material regions in the gradient scaffolds was observed. Cell culture in mineralization medium is known to increase the osteogenic differentiation and the ALP activity levels are often considered as an early marker indicating osteogenic differentiation (57). Only 300PEOT55PBT45 scaffolds supported an increased ALP activity compared to the gradient constructs and the other control scaffolds after 28 days under osteogenic conditions. WG scaffolds seemed to have an effect on GAG deposition after 7 days under chondrogenic conditions. This effect was not visible anymore at 28 days, however, when the single-material scaffolds showed a greater amount of GAG compared to 
both WG and SG scaffolds. To elucidate the effect of each surface on hMSC differentiation, a partition analysis was performed. According to previous studies, a trend in differentiation driven by the differences in polymer stiffness was expected. In 2011 Dupont et al. described the role of YAP and TAZ, transducers of the Hippo signaling pathway, in the mechanotransduction and osteogenic differentiation of MSC adhering on stiffer substrates (58). When growing on a stiff substrate, the YAP/TAZ localization was predominantly nuclear, whereas on softer substrate was cytoplasmic. The YAP/TAZ complex works as transcription factor and is involved in the osteogenic differentiation of hMSCs, by activating the Hippo pathway through modulating the cytoskeletal tension. On stiffer substrates hMSCs are more spread with a higher number of stress fibers, resulting in higher cytoskeletal tension that is expected to cause the translocation of YAP/TAZ in the nucleus and enhances the expression of osteogenic related genes.

While it is known that the stiffness of cell culture substrates can influence stem cell fate in $2 \mathrm{D}$ on soft hydrogels varying in the range between $0,1-100 \mathrm{kPa}(45)$, limited knowledge is available in $3 \mathrm{D}$ and still circumscribed to hydrogels (43) where cells are embedded in biomaterial. In case of stiffer scaffolds where cells are able to freely interact with the underneath biomaterial and to populate the pore network, much less is known. We have recently shown that hMSCs are able to sense the different stiffness of $3 \mathrm{D}$ scaffolds made of PCL with different molecular weight: lower molecular weight, associated to a lower stiffness in the MPa range, resulted in a higher hypertrophic chondrogenic differentiation (44). In this study, in SG scaffolds the softer 300PEOT55PBT45 showed the highest ALP activity after 7 days under osteogenic conditions. In WG scaffolds no major differences could be seen among the materials at 7 days, but at 28 days 300PEOT55PBT45 again showed the highest ALP activity level with respect to the other materials. Under chondrogenic conditions the materials within the SG scaffolds did not show any major difference in GAG amount at 7 and 28 days. In WG scaffolds, however, the stiffer PLA region exhibited greater GAG amount with respect to the other materials after 28 days of chondrogenic culture. A possible explanation to the results may rely in the differentiation activation of soluble factors and mechanical cues. The differentiation media have the purpose of forcing the cell differentiation toward a 
certain lineage. Culture media and growth factors were refreshed every 2 days, affecting cell differentiation; hence cell shape and cytoskeleton organization. Plausibly, soluble factors have a stronger effect on hMSC differentiation compared to mechanical cues in the stiffness range that was here considered. Additionally to soluble factors and mechanical properties, the chemistry of the different materials used and, in the specific case of WG scaffolds the gradual surface energy variation in the constructs, may have played a role in steering ALP activity and GAG production.

Further studies are needed to better characterize the effect of graded substrate stiffness and surface energy on stem cell activity at a molecular level. From an engineering perspective, a more continuous gradient could be obtained by changing the biomaterial composition every layer or by feeding two or more compositions into the extrusion chamber of the $3 \mathrm{D}$ printer with controlled and tailorable feeding rates. While the first option would be directly applicable to current AM technology, the second approach would require the design and fabrication of new printer heads. From a biological perspective, a longer study in basic conditions with more intermediate time points may highlight the solely effect of the substrate on which hMSCs are growing on. Additionally the "priming" of hMSCs on stiff polystyrene cell culture substrates, a needed step to expand cells and obtain a sufficient number before scaffold seeding, might be a critical factor. Yang et al., in fact, proved that hMSCs have a so called "mechanical memory' (59), since their culture for long period on stiff substrate may alter their differentiation ability hampering those lineages triggered by a soft substrate. Furthermore, in vivo studies will also be conducted in the future to confirm these findings in a more preclinical setting. Nonetheless, the possibility to fabricate 3D printed scaffolds with multiple materials components and changing the sequence with which specific physico-chemical properties are presented to cells seems to be an exciting new strategy to engineer material gradients in scaffolds' design and may contribute to further steer tissue formation. 


\section{Conclusion}

Additive manufacturing was successfully used to fabricate scaffolds displaying either a stiffness or a surface energy discrete gradient by depositing multiple biodegradable biomaterials in different sequential orders. While stiffness gradients didn't seem to affect the behavior of seeded hMSCs in the investigated range, surface energy gradients resulted in enhanced early osteogenic differentiation on PEOT/PBT copolymer regions and improved chondrogenic differentiation in PLA regions. The creation of scaffolds with built-in engineered physico-chemical gradients could therefore be an appealing novel strategy in the design of $3 \mathrm{D}$ scaffolds for tissue regeneration applications.

\section{Acknowledgements}

This project was funded by the Dutch Technology Foundation STW (Grant number 11135). This project/research has been also made possible with the support of the Dutch Province of Limburg. Some of the materials used in this work were provided by the Texas A\&M Health Science Center College of Medicine Institute for Regenerative Medicine at Scott \& White through a grant from NCRR of the NIH (Grant \#P40RR017447). 


\section{References}

1. Eyre D. Collagen of articular cartilage. Arthritis research. 2002;4(1):30-5. PubMed PMID: 11879535. Pubmed Central PMCID: PMC128915. Epub 2002/03/07. eng.

2. Ferguson VL, Bushby AJ, Boyde A. Nanomechanical properties and mineral concentration in articular calcified cartilage and subchondral bone. Journal of Anatomy. 2003 03/26/accepted;203(2):191-202. PubMed PMID: PMC1571155.

3. Grogan SP, Duffy SF, Pauli C, Koziol JA, Su AI, D'Lima DD, et al. Zone-specific gene expression patterns in articular cartilage. Arthritis and rheumatism. 2013 Feb;65(2):418-28. PubMed PMID: 23124445. Pubmed Central PMCID: PMC3558601. Epub 2012/11/06. eng.

4. Ghazavi MT, Pritzker KP, Davis AM, Gross AE. FRESH OSTEOCHONDRAL ALLOGRAFTS FOR POST-TRAUMATIC OSTEOCHONDRAL DEFECTS OF THE KNEE. Journal of Bone \& Joint Surgery, British Volume. 1997 November 1, 1997;79-B(6):1008-13.

5. Muller S, Breederveld RS, Tuinebreijer WE. Results of osteochondral autologous transplantation in the knee. The open orthopaedics journal. 2010;4:111-4. PubMed PMID: 20361003. Pubmed Central PMCID: PMC2847821. Epub 2010/04/03. eng.

6. Bhosale AM, Richardson JB. Articular cartilage: structure, injuries and review of management. British medical bulletin. 2008;87:77-95. PubMed PMID: 18676397. Epub 2008/08/05. eng.

7. Buckwalter JA, Mow VC, Ratcliffe A. Restoration of Injured or Degenerated Articular Cartilage. The Journal of the American Academy of Orthopaedic Surgeons. 1994 Jul;2(4):192-201. PubMed PMID: 10709009. Epub 1994/07/01. Eng.

8. Hunziker EB. Articular cartilage repair: basic science and clinical progress. A review of the current status and prospects. Osteoarthritis and Cartilage. 2002 6//;10(6):432-63.

9. Slivka MA, Leatherbury NC, Kieswetter K, Niederauer GG. Porous, resorbable, fiberreinforced scaffolds tailored for articular cartilage repair. Tissue engineering. 2001 Dec;7(6):767-80. PubMed PMID: 11749733. Epub 2001/12/26. eng.

10. Heymer A, Bradica G, Eulert J, Noth U. Multiphasic collagen fibre-PLA composites seeded with human mesenchymal stem cells for osteochondral defect repair: an in vitro study. Journal of tissue engineering and regenerative medicine. 2009 Jul;3(5):389-97. PubMed PMID: 19434664.

11. Iwan Zein DWH, *, Kim Cheng Tan, Swee Hin Teoh. Fused deposition modeling of novel scaffold architectures for tissue engineering applications. Biomaterials. 2001.

12. Ding C, Qiao Z, Jiang W, Li H, Wei J, Zhou G, et al. Regeneration of a goat femoral head using a tissue-specific, biphasic scaffold fabricated with CAD/CAM technology. Biomaterials. 2013 Sep;34(28):6706-16. PubMed PMID: 23773816. Epub 2013/06/19. eng.

13. Liu X, Liu S, Liu S, Cui W. Evaluation of oriented electrospun fibers for periosteal flap regeneration in biomimetic triphasic osteochondral implant. Journal of biomedical materials research Part B, Applied biomaterials. 2014 Oct;102(7):1407-14. PubMed PMID: 24644257. Epub 2014/03/20. eng. 
14. Claase MB, Olde Riekerink MB, de Bruijn JD, Grijpma DW, Engbers GHM, Feijen J. Enhanced Bone Marrow Stromal Cell Adhesion and Growth on Segmented Poly(ether ester)s Based on Poly(ethylene oxide) and Poly(butylene terephthalate). Biomacromolecules. 2002 2003/01/01;4(1):5763.

15. T.B.F. WOODFIELD CAVB, J. DE WIJN, T.J. SIMS, A.P. HOLLANDER and J. RIESLE. Polymer Scaffolds Fabricated with Pore-Size Gradients as a Model for Studying the Zonal Organization within Tissue-Engineered Cartilage Constructs. Tissue engineering. 2005;11(9/10).

16. Salerno A, lannace S, Netti PA. Graded biomimetic osteochondral scaffold prepared via $\mathrm{CO} 2$ foaming and micronized $\mathrm{NaCl}$ leaching. Materials Letters. 2012 9/1/;82(0):137-40.

17. Leatrese D. Harris B-SK, David J. Mooney. Open pore biodegradable matrices formed with gas foaming. 1998.

18. Levingstone TJ, Matsiko A, Dickson GR, O'Brien FJ, Gleeson JP. A biomimetic multi-layered collagen-based scaffold for osteochondral repair. Acta biomaterialia. 2014 May;10(5):1996-2004. PubMed PMID: 24418437.

19. DeLise AM, Fischer L, Tuan RS. Cellular interactions and signaling in cartilage development. Osteoarthritis and Cartilage. 2000 9//;8(5):309-34.

20. de Crombrugghe B, Lefebvre V, Nakashima K. Regulatory mechanisms in the pathways of cartilage and bone formation. Current Opinion in Cell Biology. 2001 12/1/;13(6):721-8.

21. Provot S, Schipani E. Molecular mechanisms of endochondral bone development. Biochemical and Biophysical Research Communications. 2005 3/18/;328(3):658-65

22. Deans RJ, Moseley AB. Mesenchymal stem cells: Biology and potential clinical uses. Experimental Hematology. 2000 8//;28(8):875-84.

23. Abdallah BM, Kassem M. Human mesenchymal stem cells: from basic biology to clinical applications. Gene therapy. 2008 Jan;15(2):109-16. PubMed PMID: 17989700.

24. Johnson K, Zhu S, Tremblay MS, Payette JN, Wang J, Bouchez LC, et al. A stem cell-based approach to cartilage repair. Science. 2012 May 11;336(6082):717-21. PubMed PMID: 22491093.

25. Bara JJ, Richards RG, Alini M, Stoddart MJ. Concise review: Bone marrow-derived mesenchymal stem cells change phenotype following in vitro culture: implications for basic research and the clinic. Stem cells. 2014 Jul;32(7):1713-23. PubMed PMID: 24449458.

26. Preeti Malladi YX, Michael Chiou, Amato J. Giaccia, and Michael T. Longaker. Effect of reduced oxygen tension on chondrogenesis and osteogenesis in adipose-derived mesenchymal cells. Am J Physiol Cell Physiol. 2006;290:1139-46.

27. Kilian KA, Bugarija B, Lahn BT, Mrksich M. Geometric cues for directing the differentiation of mesenchymal stem cells. Proceedings of the National Academy of Sciences. 2010 March 16, 2010;107(11):4872-7.

28. Knychala J, Bouropoulos N, Catt CJ, Katsamenis OL, Please CP, Sengers BG. Pore geometry regulates early stage human bone marrow cell tissue formation and organisation. Annals of biomedical engineering. 2013 May;41(5):917-30. PubMed PMID: 23404072. 
29. Lavery K, Swain P, Falb D, Alaoui-Ismaili MH. BMP-2/4 and BMP-6/7 differentially utilize cell surface receptors to induce osteoblastic differentiation of human bone marrow-derived mesenchymal stem cells. The Journal of biological chemistry. 2008 Jul 25;283(30):20948-58. PubMed PMID: 18436533. Pubmed Central PMCID: 3258927.

30. Lu H, Kawazoe N, Kitajima T, Myoken Y, Tomita M, Umezawa A, et al. Spatial immobilization of bone morphogenetic protein-4 in a collagen-PLGA hybrid scaffold for enhanced osteoinductivity. Biomaterials. 2012 Sep;33(26):6140-6. PubMed PMID: 22698726.

31. Re'em T, Witte F, Willbold E, Ruvinov E, Cohen S. Simultaneous regeneration of articular cartilage and subchondral bone induced by spatially presented TGF-beta and BMP-4 in a bilayer affinity binding system. Acta biomaterialia. 2012 9//;8(9):3283-93.

32. Nishimura R, Hata K, Matsubara T, Wakabayashi M, Yoneda T. Regulation of bone and cartilage development by network between BMP signalling and transcription factors. Journal of biochemistry. 2012 Mar;151(3):247-54. PubMed PMID: 22253449. Epub 2012/01/19. eng.

33. Barry F, Boynton RE, Liu B, Murphy JM. Chondrogenic differentiation of mesenchymal stem cells from bone marrow: differentiation-dependent gene expression of matrix components. Experimental cell research. 2001 Aug 15;268(2):189-200. PubMed PMID: 11478845.

34. Chen J, Chen H, Li P, Diao H, Zhu S, Dong L, et al. Simultaneous regeneration of articular cartilage and subchondral bone in vivo using MSCs induced by a spatially controlled gene delivery system in bilayered integrated scaffolds. Biomaterials. 2011 7//;32(21):4793-805.

35. McBeath R, Pirone DM, Nelson CM, Bhadriraju K, Chen CS. Cell Shape, Cytoskeletal Tension, and RhoA Regulate Stem Cell Lineage Commitment. Developmental Cell. 2004 4//;6(4):48395.

36. Even-Ram S, Artym V, Yamada KM. Matrix control of stem cell fate. Cell. 2006 Aug 25;126(4):645-7. PubMed PMID: 16923382.

37. Wei J, Igarashi T, Okumori N, Igarashi T, Maetani T, Liu B, et al. Influence of surface wettability on competitive protein adsorption and initial attachment of osteoblasts. Biomedical materials. 2009 Aug;4(4):045002. PubMed PMID: 19525576.

38. Zanetti NC, Solursh M. Induction of chondrogenesis in limb mesenchymal cultures by disruption of the actin cytoskeleton. The Journal of cell biology. 1984 Jul;99(1 Pt 1):115-23. PubMed PMID: 6539780. Pubmed Central PMCID: PMC2275608. Epub 1984/07/01. eng.

39. Guilak F, Cohen DM, Estes BT, Gimble JM, Liedtke W, Chen CS. Control of Stem Cell Fate by Physical Interactions with the Extracellular Matrix. Cell Stem Cell. 2009 7/2/;5(1):17-26.

40. Chatterjee K, Lin-Gibson S, Wallace WE, Parekh SH, Lee YJ, Cicerone MT, et al. The effect of 3D hydrogel scaffold modulus on osteoblast differentiation and mineralization revealed by combinatorial screening. Biomaterials. 2010 Jul;31(19):5051-62. PubMed PMID: 20378163. Pubmed Central PMCID: 3125577.

41. Dupont S, Morsut L, Aragona M, Enzo E, Giulitti S, Cordenonsi M, et al. Role of YAP/TAZ in mechanotransduction. Nature. 2011 Jun 9;474(7350):179-83. PubMed PMID: 21654799. 
42. Park JS, Chu JS, Tsou AD, Diop R, Tang Z, Wang A, et al. The effect of matrix stiffness on the differentiation of mesenchymal stem cells in response to TGF-beta. Biomaterials. 2011 Jun;32(16):3921-30. PubMed PMID: 21397942. Pubmed Central PMCID: PMC3073995. Epub 2011/03/15. eng.

43. Huebsch N, Arany PR, Mao AS, Shvartsman D, Ali OA, Bencherif SA, et al. Harnessing traction-mediated manipulation of the cell/matrix interface to control stem-cell fate. Nature materials. 2010 Jun;9(6):518-26. PubMed PMID: 20418863. Pubmed Central PMCID: 2919753. Epub 2010/04/27. eng.

44. Hendrikson WJ, Rouwkema J, van Blitterswijk CA, Moroni L. Influence of PCL molecular weight on mesenchymal stromal cell differentiation. Rsc Adv. 2015;5(67):54510-6. PubMed PMID: WOS:000356865500067. English.

45. Engler AJ, Sen S, Sweeney HL, Discher DE. Matrix elasticity directs stem cell lineage specification. Cell. 2006 Aug 25;126(4):677-89. PubMed PMID: 16923388. eng.

46. McBeath R, Pirone DM, Nelson CM, Bhadriraju K, Chen CS. Cell shape, cytoskeletal tension, and RhoA regulate stem cell lineage commitment. Developmental cell. 2004 Apr;6(4):483-95. PubMed PMID: 15068789. Epub 2004/04/08. eng.

47. Reichert JC, Cipitria A, Epari DR, Saifzadeh S, Krishnakanth P, Berner A, et al. A tissue engineering solution for segmental defect regeneration in load-bearing long bones. Science translational medicine. 2012 Jul 4;4(141):141ra93. PubMed PMID: 22764209.

48. Ge Z, Tian X, Heng BC, Fan V, Yeo JF, Cao T. Histological evaluation of osteogenesis of 3Dprinted poly-lactic-co-glycolic acid (PLGA) scaffolds in a rabbit model. Biomedical materials. 2009 Apr;4(2):021001. PubMed PMID: 19208943.

49. Bartha L, Hamann D, Pieper J, Peters F, Riesle J, Vajda A, et al. A clinical feasibility study to evaluate the safety and efficacy of PEOT/PBT implants for human donor site filling during mosaicplasty. European journal of orthopaedic surgery \& traumatology : orthopedie traumatologie. 2013 Jan;23(1):8191. PubMed PMID: 23412412.

50. Emans PJ, Jansen EJ, van lersel D, Welting TJ, Woodfield TB, Bulstra SK, et al. Tissueengineered constructs: the effect of scaffold architecture in osteochondral repair. Journal of tissue engineering and regenerative medicine. 2013 Sep;7(9):751-6. PubMed PMID: 22438217.

51. Athanasiou KA, Zhu C, Lanctot DR, Agrawal CM, Wang X. Fundamentals of biomechanics in tissue engineering of bone. Tissue Eng. 2000 Aug;6(4):361-81. PubMed PMID: 10992433.

52. Mow VC, Ratcliffe A, Rosenwasser MP, Buckwalter JA. Experimental studies on repair of large osteochondral defects at a high weight bearing area of the knee joint: a tissue engineering study. $J$ Biomech Eng. 1991 May;113(2):198-207. PubMed PMID: 1875694.

53. Yeung T, Georges PC, Flanagan LA, Marg B, Ortiz M, Funaki M, et al. Effects of substrate stiffness on cell morphology, cytoskeletal structure, and adhesion. Cell motility and the cytoskeleton. 2005 Jan;60(1):24-34. PubMed PMID: 15573414. Epub 2004/12/02. eng.

54. Zemel A, Rehfeldt F, Brown AE, Discher DE, Safran SA. Cell shape, spreading symmetry and the polarization of stress-fibers in cells. Journal of physics Condensed matter : an Institute of Physics journal. 2010 May 19;22(19):194110. PubMed PMID: 20458358. Pubmed Central PMCID: 2865697. 
55. Brynda E, Hlady V, Andrade JD. Protein Packing in Adsorbed Layers Studied by ExcitationEnergy Transfer. Journal of Colloid and Interface Science. 1990 Oct 15;139(2):374-80. PubMed PMID: WOS:A1990EG52600009. English.

56. Tamada Y, Ikada Y. Effect of Preadsorbed Proteins on Cell Adhesion to Polymer Surfaces. Journal of Colloid and Interface Science. 1993 2//;155(2):334-9.

57. Langenbach F, Handschel J. Effects of dexamethasone, ascorbic acid and betaglycerophosphate on the osteogenic differentiation of stem cells in vitro. Stem cell research \& therapy. 2013;4(5):117. PubMed PMID: 24073831. Pubmed Central PMCID: PMC3854789. Epub 2013/10/01. eng.

58. MacQueen L, Sun Y, Simmons CA. Mesenchymal stem cell mechanobiology and emerging experimental platforms. Journal of the Royal Society, Interface / the Royal Society. 2013 Jul 6;10(84):20130179. PubMed PMID: 23635493. Pubmed Central PMCID: 3673151. Epub 2013/05/03. eng.

59. Yang C, Tibbitt MW, Basta L, Anseth KS. Mechanical memory and dosing influence stem cell fate. Nat Mater. 2014 06//print;13(6):645-52. 


\section{CHAPTER 7}

\section{Creeping Proteins in Microporous Structures: Polymer Brush-Assisted Fabrication of 3D Gradients for Tissue Engineering}

Michel Klein Gunnewiek ${ }^{\dagger}$, Andrea Di Luca ${ }^{\dagger}$, Hermannes Z. Bollemaat, Clemens, A. van Blitterswijk, G. Julius Vancso, Lorenzo Moroni* and Edmondo M. Benetti*

† These authors contributed equally to this work

* These authors contributed equally to this work.

Published on Adv Healthc Mater. 2015 Jun 3;4(8):1169-74 
Recent research in tissue engineering and regenerative medicine is increasingly revolving around effective fabrication techniques to create functional scaffolds for cell manipulations. Specifically, 3D supports presenting temporal and spatial control over the exposure of protein cues are desirable as they would allow spatial control over cell behavior. ${ }^{[1,2]}$ To achieve this objective, a number of methods have been proposed to create synthetic extra-cellular matrices (ECMs) with gradient-like chemical compositions, thus mimicking the continuous variation characteristic of natural ECM.

The production of biomolecular gradients on 2D supports was proven as an effective approach to spatially adjust cell adhesion, migration and proliferation on planar substrates. ${ }^{[3-7]}$ Following the development of increasingly sophisticated $3 \mathrm{D}$ synthetic ECMs, diverse methodologies for the fabrication of protein gradients in $3 D$ environments have been also proposed. These biomaterials are obtained by employing hydrogel-supports ${ }^{[8-11]}$ and electrospun fibers ${ }^{[12,13]}$ but they commonly require complicated multi-steps preparations without achieving spatial control of the biomolecules.

The simple exposure of 3D supports to different protein solutions with variable composition ${ }^{[14,15]}$ consented the production of biomaterials showing a continuous variation of protein coverage but just along one main scaffold axis. Following such preparations, electrospun fibers with varying composition could be deposited on a movable stage while changing feed, in order to create (bio)chemical gradients along the horizontal or the vertical direction.

Protein gradients were alternatively fabricated within photo-crosslinked hydrogels by gradually or locally exposing different parts of the matrix to light ${ }^{[8,9]}$, followed by bio-conjugation. Analogous mono-axial hydrogel-supported gradients were also successfully produced by electrochemically-controlled enzymatic polymerization ${ }^{[16]}$. Higher control over the compositional changes characterizing synthetic ECMs was accomplished via layer-by-layer ${ }^{[10]}$ or "gradient maker"-assisted ${ }^{[17-19]}$ fabrications. In these processes hydrogels presenting gradient compositions along their deposition/building direction were formed. ${ }^{[10,20]}$

The above-mentioned methods yielding gradient compositions necessitated multiple processing steps and/or time-consuming chemical treatments. These 
drawbacks would render them unsuitable for clinical use where fast and low-cost manipulations to reproduce and directly apply scaffolds on patients are needed. In addition, these techniques supported the formation of mono- or bi-directional gradients, while the development of concentration changes in 3D still remained a challenge.

Synthetic supports which mimicked the multi-axial compositional gradients, characteristic of natural ECM in vivo, would be needed to reproduce the compositional diversity of tissue environments within simplified and easily accessible matrices. These supports could be applied not only as scaffolds for the regeneration of complex tissues, but also as $3 \mathrm{D}$ architectures for studying cells and bacteria adhesion and migration ${ }^{[9,12,16]}$ in $3 \mathrm{D}$ environments.

Triggered by this challenge, we introduce here a novel fabrication strategy which features a practical and affordable construction of 3D ECMs that display multidirectional variations of (bio)chemical environments. Specifically, we coupled rapid prototyping $(\mathrm{RP})^{[21-23]}$ to surface modification strategies based on surface-initiated polymerizations $(\mathrm{SIP})^{[24,25]}$ in order to fabricate microporous and highly-functional $3 \mathrm{D}$ architectures which could be subsequently locally decorated with different types of biomolecules. In this process, poly( $\varepsilon$-caprolactone) (PCL) scaffolds characterized by a regularly layered network of microfibers (Scheme 1a) were modified by surface-initiated atom transfer radical polymerization (SI-ATRP) of poly(oligo (ethylene glycol) methacrylate) (POEGMA) (Scheme 1b). The so-formed, sub-100 nm POEGMA brushes uniformly covered the scaffold surface and allowed protein coupling at the exposed hydroxyl functions of the grafted polymer ${ }^{[26,27]}$ (Scheme 1c). 


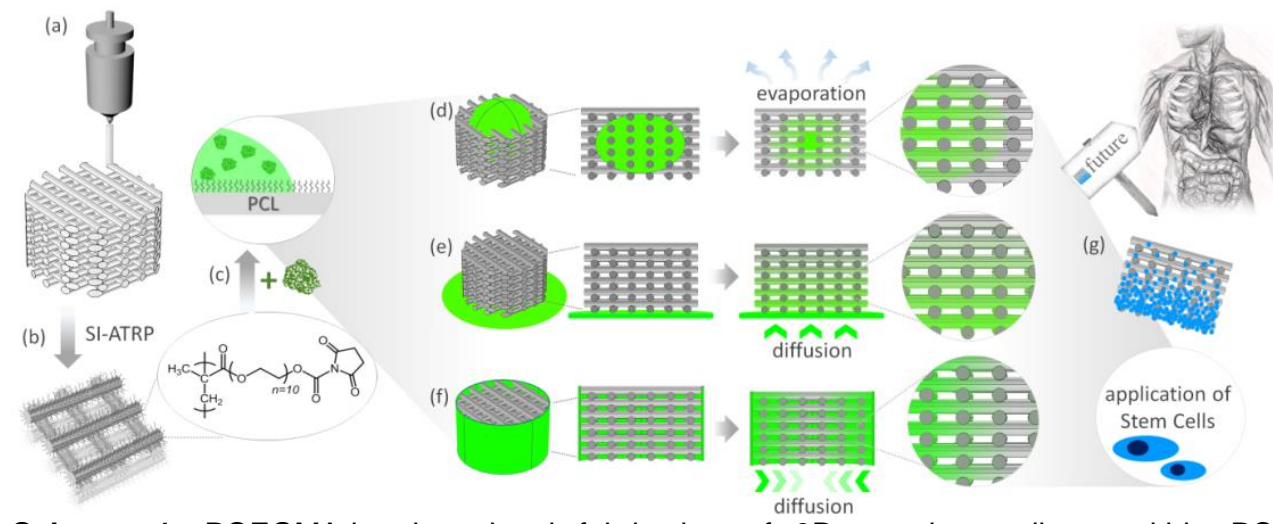

Scheme 1. POEGMA-brush-assisted fabrication of 3D protein gradients within PCL scaffolds and application as platforms for stem cells immobilization. (a) fabrication of PCL microporous scaffolds by rapid prototyping; (b) SI-ATRP of OEGMA from the PCL fibers network and subsequent activation of hydroxyl side chains to form NHS esters; (c) conjugation of proteins at the brush interface by controlled diffusion of solutions within the 3D scaffolds. This last step is especially highlighted in (d), (e) and (f). Incorporation of microdroplets of protein solutions and subsequent solvent evaporation generated radial concentration gradients on brushes (d). Controlled diffusion from a soaked paper reservoir allowed the formation of axial protein concentration gradients (e). Wrapped reservoirs enabled protein diffusion from the lateral walls of the scaffolds and the consequent fabrication of radial protein gradient developing oppositely to (d). The protein-functionalized scaffolds were finally applied for the spatially controlled immobilization of hMSCs $(\mathrm{g})$.

In addition, the biopassive character of POEGMA brushes ${ }^{[28-30]}$ assured robust covalent linkage of cues avoiding any further unspecific contamination of the matrix by physisorption of other biomolecules or bacteria attachment. The formation of protein gradients by surface conjugation was accomplished exploiting the physicochemical properties of the hydrophilic brush in combination with the microporous structure of the PCL scaffolds. Uniform coverage of PCL by hydrophilic POEGMA brush caused a marked increase in wettability of the support (water contact angle (CA) varied from $73^{\circ}$ to $45 \pm 3^{\circ}$, as shown in Table S1). This phenomenon reflected a substantial increase of surface energy within the $3 D$ scaffold. ${ }^{[31,32]}$ Hence, the interplay between high surface energy-driven wetting by the POEGMA brush and capillary forces within the microporous supports promoted the diffusion of aqueous solutions into the matrix (as highlighted in Scheme 1d and in the light microscope images shown in Figure S1). We exploited this simple process to incorporate protein solutions into the scaffolds and consequently couple proteins at the preactivated brush (via NHS chemistry ${ }^{[33]}$ as described in the Experimental Section). 
Diffusion of protein solution micro-droplets (Scheme 1d) and subsequent concentration of the solutions towards the inner core of the structure finally induced a radial variation of protein surface concentration (Figure 1a-c). Alternatively, protein solutions could be made to diffuse from soaked paper sheets used as solution reservoir put into close contact with the scaffolds (Scheme 1e and Figure 1d-f). In both cases, simple process parameters like microdroplets volume and diffusion time from external reservoirs allowed spatial 3D control over the concentration of the coupled proteins (Figures S2 and S3).
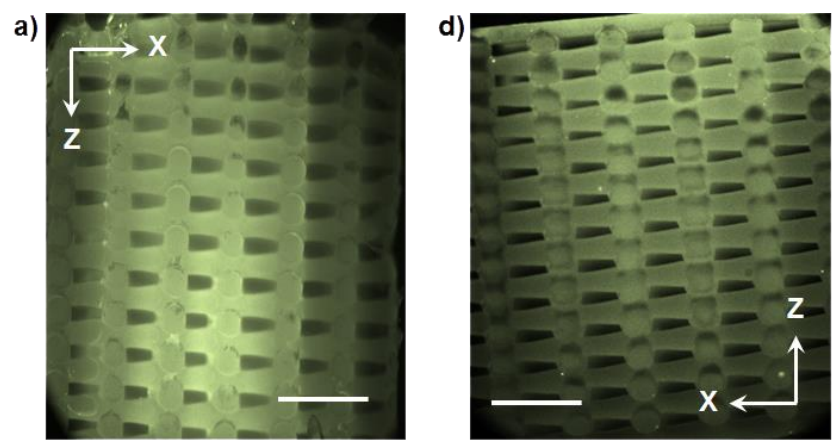

b)
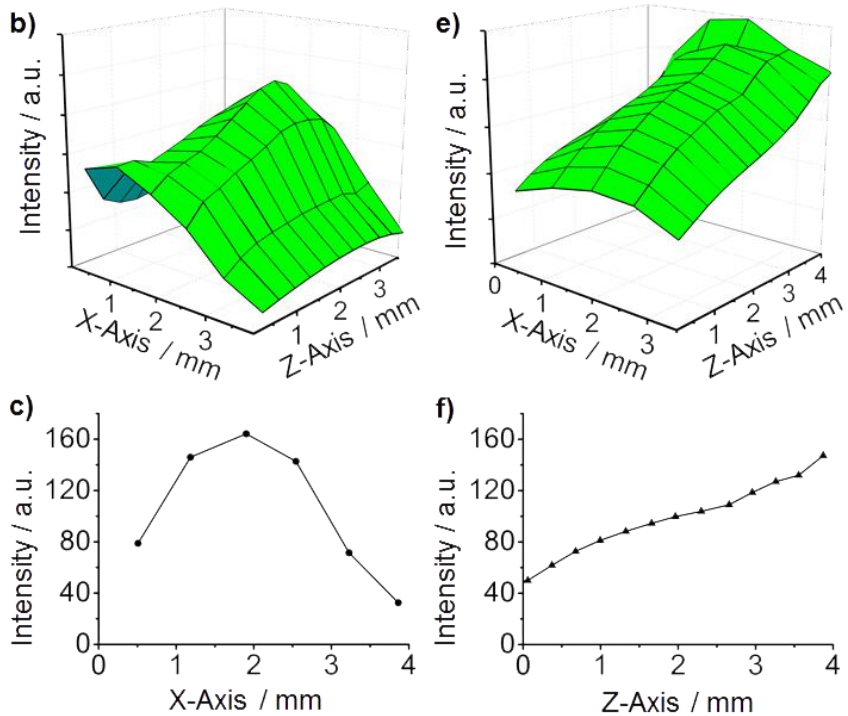

Figure 1. Radial and axial 3D gradients of proteins within POEGMA brush-coated microporous scaffolds. Fluorescent micrographs $(a, d)$ and the corresponding 3D intensity profile $(b, e)$ of the vertical cross-section of PCL-POEGMA scaffolds functionalized with a radial (a-c) and an axial (d-f) gradient of BSA-FITC concentration. Intensity profiles along the $x$-axis (c) and the $z$-axis (f) of the radial and the axial 3D gradient, respectively. 
Figure 1a-c shows radial 3D gradients of fluorescently labeled bovine serum (BSA) attached to PCL-POEGMA scaffolds by incorporation of protein solution microdroplets and subsequent bio-conjugation. Having calculated through Equation 1 the inner volume of each scaffold as $25 \mu \mathrm{l}$, we applied different volumes of microdroplets in order to prepare radial gradients, yielding gradient protein coverages. Fluorescent microscopy of the cross-sectioned 3D structures revealed the protein surface concentration profiles within the scaffolds. As shown in Figure S2a-b, gradient formation from $2 \mu$ protein solutions produced a radial gradient with a high concentration of proteins in the inner core and a steady decrease of coverage after around $250 \mu \mathrm{m}$ from the center of the scaffolds. Increase of the microdroplet volume to $10 \mu \mathrm{l}$ produced a more uniform protein coverage through the supports with just the outer walls remaining unfunctionalized (Figure 1a-c). Although the volume used for these depositions was less than half of the total inner volume of the 3D scaffolds, fast diffusion of protein solutions along the hydrophilic brush-coated fibers most likely smoothened the profile of the protein gradient across the 3D structures.

Brush-supported protein gradients developing axially along the 3D scaffold (from one external side towards the center) were obtained by applying a porous paper sheet as solution reservoir and allowing the protein medium diffuse by capillary forces (Scheme 1e, Figure 1d-f and Figure S3). Slow wetting of brush-coated fibers enabled the adjustment of the protein gradient profile as a function of the diffusion time (and thus bio-conjugation time at the POEGMA brush surface).

The fluorescent images in Figure S3 showed different axial gradient profiles obtained by varying the diffusion/bio-conjugation time. Both $3 \mathrm{D}$ and the $2 \mathrm{D}$ intensity plots reconstructed from the fluorescence micrographs showed that, after 30 minutes of diffusion, proteins were mainly immobilized within $700 \mu \mathrm{m}$ from the base of the scaffold (Figure S3a-b). Following longer diffusion time, protein solutions covered larger volumes inside the scaffolds, homogenizing the protein coverage. After 60 minutes of diffusion (Figures S3c-d) proteins covered almost 1/3 of the distance across the scaffold main axis ( $Z$ axis in Figure $S 3 g$ ), keeping a rather uniform surface concentration along the $X$ axis (Figure S4). After 120 minutes of diffusion the protein solutions completely wetted the inner structure of 
the scaffolds and consequently created a nearly linear gradient of protein coverage on the fibers (Figure 1d-f).

The procedures used for the fabrication of single protein gradients were combined to produce double gradients of different protein species. After the formation of either a radial or an axial 3D distribution of proteins the scaffolds were not entirely covered by protein solutions. Consequently, unfunctionalized POEGMA brushes were still available for bio-conjugation on the unwetted areas within the $3 \mathrm{D}$ structure. Hence, a second protein solution could be additionally applied to produce a surface concentration gradient which developed in the opposite direction to the pre-existing one (as schematized in Scheme 1d-f). These "double" protein distributions are referred to as 3D radial and axial double gradients. In order to ease their analysis by fluorescent microscopy, we applied two solutions of differently labeled BSA (FITC and Texas Red, named as protein A and B, respectively) as representative of two different protein species. As shown in Figure $2 \mathrm{a}-\mathrm{d}$, a $3 \mathrm{D}$ radial double gradient of two different proteins was produced first incorporating a microdroplet of protein A solution within the POEGMA-PCL scaffold and subsequently wrapping around its outer surface a paper reservoir soaked with protein B. Alternatively, in order to fabricate a 3D axial double gradient of protein A and $B$, two different protein media were allowed to diffuse from two reservoirs placed in contact with each opposite "face" of the scaffold (Figure 2e-h).
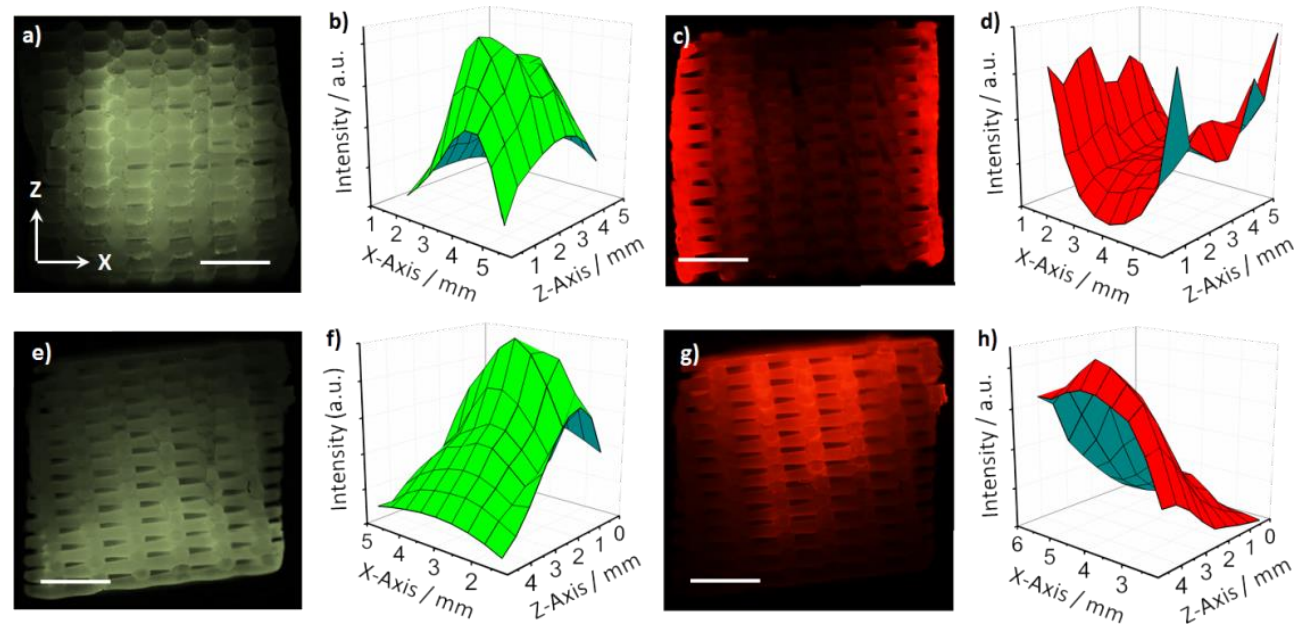
Figure 2. Double 3D gradients of proteins within POEGMA brush-coated microporous scaffolds. Fluorescent micrographs $(a, c, e, g)$ and the corresponding intensity plots $(b, d, f, h)$ of PCL-POEGMA scaffolds functionalized with BSA. Double gradients in BSA concentrations were fabricated both in the radial and axial directions; $(a, b)$ inside-to-outside and (c,d) outside-to-inside; (e,f) bottom-to-top and $(\mathrm{g}, \mathrm{h})$ top-to-bottom. Scale bar is $1 \mathrm{~mm}$.

As shown in Figures $2 \mathrm{a}$ and $2 \mathrm{c}$ and in the corresponding $3 \mathrm{D}$ intensity reconstructions from a typical functionalized scaffold, radial double gradients displayed continuous and interconnected variations of concentrations of proteins $A$ and $B$. The radial double gradients typically presented a high concentration of protein $A$ in the core of the scaffold, which decreased beyond around $1.5 \mathrm{~mm}$ towards the outer part of the scaffold (Figure $2 \mathrm{~b}$ ). Protein B concentrations, on the contrary, developed from the outer walls of the scaffold and showed high surface concentration within around $1 \mathrm{~mm}$ towards the interior of the support (Figure $2 \mathrm{~d}$ ).

Axial double gradients (Figures $2 \mathrm{e}-\mathrm{g}$ ) showed a uniform and interconnected variation of the two proteins concentration across the main scaffold axis $(Z$ axis in Figures $2 f$ and $2 h$ ). In these cases, a well-pronounced concentration distribution of the two protein species along opposite directions was clearly showed.

To prove the applicability of the proposed fabrications for cell manipulations we reproduced both radial and axial 3D gradients using fibronectin (FN) as a biological cue known to promote cell adhesion. Also in this case, brush coatings based on NHS-activated POEGMA were used as platforms for bio-conjugation and the successful linkage of FN was subsequently proved by X-ray photoelectron spectroscopy (XPS, Figure S5). In addition, the surface coverage of FN has been estimated to be around $40 \pm 5 \mathrm{ng} / \mathrm{cm}^{2}$. Following the controlled diffusion of $\mathrm{FN}$ solutions, functionalized scaffolds presenting a radial and an axial concentration gradient were subsequently incubated with hMSCs during 1 day. The adhered cells were subsequently stained using methylene blue and the scaffolds were sectioned along the appropriate axis in order to visualize their interior.

$3 \mathrm{D}$ scaffolds presenting a radial gradient of $\mathrm{FN}$ from the core to the outer volume of the scaffolds showed higher number of cells in the interior of the structure, while at its periphery only few cells could be visualized (Figures $3 a$ and $3 b$ ). In a similar way, axial gradients of brush-supported FN induced hMSC adhesion mainly on one side of the scaffold, according to $\mathrm{FN}$ distribution and following the gradient 
morphology (Figure 3c). Scaffolds completely functionalized with FN and "bare" POEGMA-coated supports were also seeded with hMSCs and were used as controls (Figures $3 d$ and $3 e$ ). As shown in Figure $3 d$, the cells completely covered the uniformly functionalized scaffolds without showing any preferred area to settle. In comparison, brush-coated scaffold without fibronectin showed no cells due to the biopassive nature of unfunctionalized POEGMA (Figure 3e). To investigate the viability of hMSCs adhering onto the POEGMA-FN covered 3D structures, high magnification scanning electron microscopy images were taken (Figure S6). The clear deposition of extracellular matrix (ECM) (Figure S6b-f) and the highly spread appearance of the cells (Figure S6f) are signs that the cells are healthy and vital.
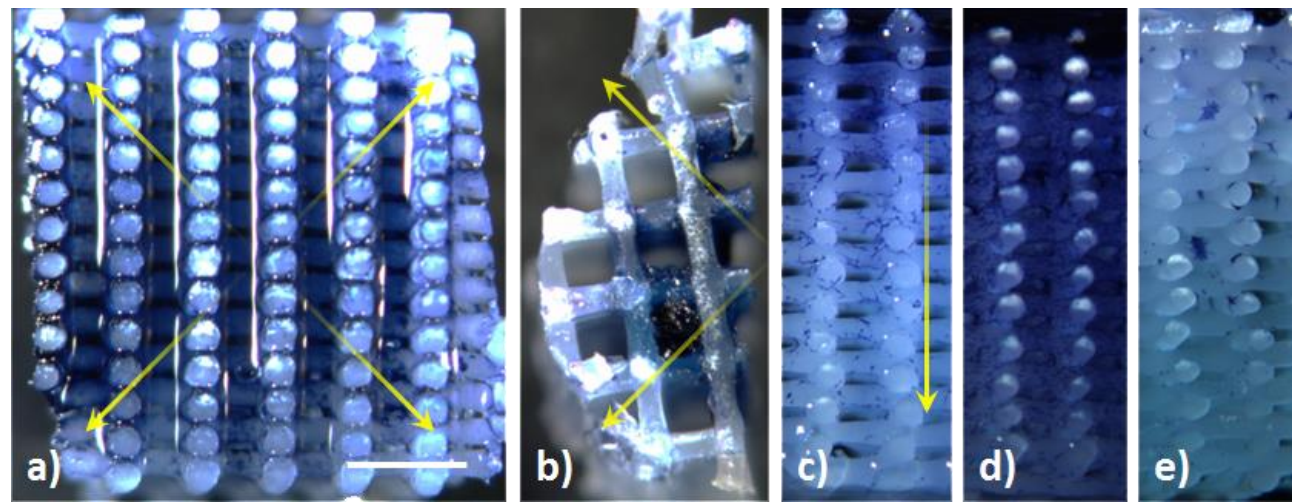

Figure 3. Optical images of methylene blue stained hMSCs adhering on PCL-POEGMA scaffolds. Optical images of methylene blue stained hMSCs adhering on PCL-POEGMA scaffolds. Radial gradient in FN concentration showing the vertical (a) and horizontal (b) cross-section; an axial gradient in FN concentration (c) a POEGMA coating fully covered by FN (d) and a bare POEGMA coating (e). The yellow arrows indicate the direction of decreasing FN concentration. Scale bar is $1 \mathrm{~mm}$.

All these results confirmed that the fabrication method proposed in this work represents a highly efficient strategy not only to create 3D protein gradients with designer morphologies, but also to control spatially cell adhesion within 3D scaffolds.

The use of hydrophilic and highly functional brush layers coupled to a structured support with controlled microporosity triggered diffusion and concomitant covalent immobilization of proteins from solutions. These processes could be easily manipulated by varying parameters as solution volumes and diffusion time within 
the $3 D$ structures. This enabled the fabrication of $3 D$ axial and radial protein gradients with tailored morphologies.

PCL-POEGMA scaffolds with 3D protein gradients have the potential to be easily reproduced also in clinics since they do not require any inert environment or complicated chemistry. Brush-coated scaffolds could be simply incubated with the required proteins and directly applied to patients in or without the presence of cell preparations. In this context, the versatility of this technique would support the combination of multiple cues ${ }^{[34-38]}$ that could eventually trigger cell adhesion, migration or differentiation, in a single 3D porous structure. All the abovementioned features make the method presented here a practical and affordable strategy to synthetically mimic natural ECMs and their 3D multidirectional diversity.

\section{Experimental Section}

Materials: Oligo(ethylene glycol) methacrylate (OEGMA, Aldrich, $\mathrm{Mn}=526 \mathrm{~g} / \mathrm{mol}$ ) was purified from hydrochinone inhibitors by passing it through a basic alumina column using dichloromethane (DCM, Biosolve) as eluent. Afterwards DCM was removed under vacuum. Copper $(\mathrm{I})$ chloride ( $\mathrm{CuCl}$, Aldrich, $98 \%$ ) was purified by stirring in glacial acetic acid, filtering, and washing with ethanol three times, followed by drying in vacuum at room temperature overnight. Poly( $\varepsilon$-caprolactone) (PCL, $\mathrm{Mn}=45 \mathrm{kDa}$ ) was obtained from Sigma. Copper(II) bromide (Sigma-Aldrich, $\geq 99 \%$ ), methanol (Biosolve, absolute), isopropanol (iPA, Biosolve), ethylenediamine (EDA, Sigma-Aldrich, $\geq 99 \%$ ), dry hexane (Acros, Extra Dry over Molecular Sieve, $97 \%$ ), N,N-Dimethylformamide (DMF, Acros, Extra Dry over Molecular Sieve, 99,8 \%), pyridine (Sigma-Aldrich, anhydrous, 99,8\%), 2,2'-bipyridil (BiPy, Sigma-Aldrich, $\geq 99 \%$ ), 2-bromoisobutyryl xbromide (BIBB, Aldrich, $98 \%$ ), Ethylenediaminetetraacetic acid disodium salt dihydrate (EDTA, Sigma, $99 \%$ ), 4-dimethylaminopyridine (DMAP, Sigma-Aldrich, $\geq 99 \%$ ), N,N'disuccinimidyl carbonate (DSC, Sigma-Aldrich, $98 \%$ ), phosphate buffered saline (PBS) (Invitrogen) and triethylamine (Sigma-Aldrich, $\geq 99 \%$ ) were used as received. For brush surface modification fluorescein labeled bovine serum albumin (BSA), Texas Red labeled BSA and fibronectin (FN) were used as obtain from Invitrogen and diluted to the appropriate concentration. All water used in the 
experiments was Millipore Milli-Q grade. Colony-picked human mesenchymal stem cells hMSCs (male, age 22) were retrieved from the Institute of Regenerative Medicine (Temple, Texas). For cell culture Basic cell culture media was prepared by adding to a $\alpha$-MEM cell medium (Invitrogen) $10 \mathrm{v} / \mathrm{v} \%$ of Fetal Bovine Serum (FBS), $2 \mathrm{mM}$ of L-Glutamine, $100 \mathrm{U} / \mathrm{mL}$ of penicillin, $100 \mu \mathrm{g} / \mathrm{mL}$ of streptomycin, and $0.2 \mathrm{mM}$ of ascorbic acid. All these components were obtained from Invitrogen. Scaffold fabrication: 3D microporous PCL scaffolds were fabricated using a rapid prototyping technique (Envisiontec $\mathrm{GmbH}$, Germany). The PCL granulate was loaded into a metal syringe wrapped with a heating jacket. The copolymer was heated to $100^{\circ} \mathrm{C}$ for about $45 \mathrm{~min}$ until it had reached the molten phase and could be dispensed through the extruder. A nitrogen pressure of 5 bar was applied on top of the metal cartridge to facilitate polymer strand extrusion from a Luer Lock stainless steel needle. A rectangle block model of $4 \mathrm{~mm}$ in height and $20 \mathrm{~mm} \times 20$ $\mathrm{mm}$ in area was plotted layer-by-layer and the architecture (0-90) was controlled by the Bioplotter CAD/CAM software. The layered fibers within the scaffolds were assembled in a woodpile structure having the following characteristic parameters: diameter $\left(d_{1}\right)=250 \mu \mathrm{m}$, spacing $\left(d_{2}\right)=650 \mu \mathrm{m}$, layer thickness $\left(d_{3}\right)=150 \mu \mathrm{m}$. From these values the theoretical porosity $(\sim 50 \%)$ of the scaffold was calculated, according to:

$$
P=1-\frac{V_{\text {Scaffold }}}{V_{\text {Cube }}}=1-\frac{\pi}{4} \times \frac{d_{1}^{2}}{d_{2} \times d_{3}}(1)
$$

The 3D scaffolds used for the fabrication of the brush-supported protein gradients were cut from the rectangle block in order to obtain cylindrical shapes with a height and a diameter of $4 \mathrm{~mm}$.

Activation of the polymer films: 3D scaffolds were activated through immersion in a $5 \mathrm{mM}$ isopropanol solution of ethylenediamine (EDA). The reaction was allowed to proceed for 10 minutes at room temperature. Scaffolds were then rinsed with icecold water and finally dried in a stream of nitrogen. The aminated PCL scaffolds were immersed into $20 \mathrm{ml}$ of dry hexane and $20 \mu \mathrm{L}$ of dry pyridine, to which $20 \mu \mathrm{L}$ of 2-bromoisobutyryl bromide (BIBB) was added dropwise. The reaction mixture was gently stirred for 1 hour at room temperature to produce the 2bromoisobutyrate-PCL surface (PCL-Br). PCL-Br scaffolds were later-on washed 
repeatedly with an ethanol/water $(1 / 1, v / v)$ mixture and finally dried under a stream of nitrogen.

Atom transfer radical polymerization of OEGMA: Purified OEGMA monomer ( $5 \mathrm{~g}$, $9.5 \mathrm{mmol}$ ) and 2,2'bipyridine $(81.7 \mathrm{mg}, 0.52 \mathrm{mmol})$ were added to a water $(5 \mathrm{ml})$ and methanol $(1,26 \mathrm{ml})$ mixture. The solution was purged with argon for 30 minutes. $\mathrm{CuCl}(18.75 \mathrm{mg}, 0.19 \mathrm{mmol})$ and $\mathrm{CuBr}_{2}(2 \mathrm{mg}, 0.009 \mathrm{mmol})$ were added into another reaction flask and also flushed with argon. Monomer, ligand and catalyst were then combined and stirred for other 30 minutes to facilitate the formation of the organometallic complex. This solution was then transferred into the flasks containing PCL-Br substrates. The flasks were sealed with rubber septa and kept at room temperature under nitrogen. Following 10 minutes of reaction time the substrates were removed from the polymerization solution, exhaustively rinsed with water to remove any unreacted compound and finally dried under a stream of nitrogen. From detailed kinetics studies of SI-ATRP from PCL substrates (Supporting Information) the average thickness of POEGMA brushes following 10 minutes of polymerization resulted as $15 \mathrm{~nm}$ (Figure S7). Before any further manipulation, PCL-POEGMA scaffolds were incubated in a $0.1 \mathrm{M}$ aqueous EDTA solution overnight to remove any copper trace. In order to test the successful uniform grafting of POEGMA, functionalized scaffolds were finally placed in water containing vials (Figure S8). Pure PCL scaffolds floated due to their hydrophobic nature, while PCL-POEGMA scaffolds sank to the bottom of the vials.

Functionalization of PCL-POEGMA scaffolds: POEGMA brushes on PCL scaffolds were activated by placing them in a dry DMSO solution containing $200 \mathrm{mM}$ of DSC and DMAP. Later on, the samples were incubated in a protein solution containing either $0,4 \mu \mathrm{M}$ fluorescently labeled $\mathrm{BSA}$ or $0,1 \mu \mathrm{M} \mathrm{FN}$. To fabricate a radial gradient in protein concentration from the core to the outer surface of the $3 \mathrm{D}$ scaffolds, microdroplets of either 2 or $10 \mu \mathrm{L}$ of PBS protein solutions were placed on the scaffold and let diffuse inside their core. After 10 minutes the scaffolds were extensively rinsed with milli-q water, blow-dried with a stream of $\mathrm{N}_{2}$, cut over their mid-section and finally imaged with a fluorescent microscope. Reversed radial gradients from the outer surface of the scaffolds to the core were fabricated using microporous paper sheets soaked in PBS protein solutions as reservoirs. Soaked 
paper sheets were wrapped around the scaffolds and kept into close contact in order to let the protein solutions diffuse within the scaffolds interior. Following 3 minutes of incubation the scaffolds were extensively rinsed with milli-q water, blowdried with a stream of $\mathrm{N}_{2}$, cut over their mid-section and finally imaged with a fluorescent microscope. Protein gradients along the axial direction of the scaffolds were fabricated by placing the DSC-activated PCL-POEGMA scaffolds on top of a micro porous paper sheet previously soaked with protein solutions. The contact between the scaffold's outer surface and the paper reservoir was assured by placing a weight of $7.5 \mathrm{~g}$ on top of the scaffolds. Following different diffusion times (30, 60 and 120 minutes) the scaffolds were extensively rinsed with milli-q water, blow-dried with a stream of $\mathrm{N}_{2}$, cut over their mid-section and finally imaged with a fluorescent microscope.

Fluorescent microscopy: Fluorescent micrographs of the scaffolds functionalized with labeled BSA species were recorded using a Nikon Eclipse E600. Fluoresceinlabelled BSA was visualized using a filter with an excitation and emission wavelength of $475 \mathrm{~nm}$ and $530 \mathrm{~nm}$, respectively. For Texas Red-labelled BSA, a filter with an excitation and emission wavelength of $559 \mathrm{~nm}$ and $630 \mathrm{~nm}$, respectively, was used. Unmodified PCL scaffolds were used to set the exposure time and the gain values, such that the auto-fluorescence of the bare polymer was suppressed. Fluorescent pictures were taken from the vertical cross-section by cutting the scaffolds true the center along the z-axis. An assumption is made that the functionalization of the POEGMA layer is homogeneous and that the scaffold can be turned freely around the z-axis.

Cell culture and cell staining: hMSCs were cultured at $37^{\circ} \mathrm{C}$ in a humidified atmosphere of $5 \%$ carbon dioxide, using as culture medium a-MEM supplemented with $10 \mathrm{v} / \mathrm{v} \%$ FBS, $2 \mathrm{mM}$ L-Glutamine, $0.2 \mathrm{mM}$ L-ascorbic acid 2-phosphate magnesium salt, $100 \mathrm{U} / \mathrm{mL}$ of penicillin and $10 \mu \mathrm{g} / \mathrm{mL}$ of streptomycin. The cells were seeded at a density of 500,000 cells in $40 \mu \mathrm{L}$ per scaffold and after 4 hours, cell culture media was added. The scaffolds were kept in an incubator for one day. After the required culturing time, the cells were fixed with a $3.7 \mathrm{v} / \mathrm{v} \%$ formaldehyde solution in PBS. Subsequently the cells were stained using a $1 \%$ methylene blue solution in water and visualized using an optical microscope. 


\section{Acknowledgements}

This work was financially supported by the MESA+ Institute for Nanotechnology of the University of Twente, by the Technology foundation STW (STW, 11135) and by the Swiss National Foundation (SNSF "Ambizione" PZOOP2-148156). Some of the materials employed in this work were provided by the Texas A\&M Health Science Center College of Medicine Institute for Regenerative Medicine at Scott \& White through a grant from NCRR of the NIH (Grant \# P40RR017447). The authors thank Prof. Lucio Isa (ETHz) and Dr. Mark Hempenius (UTwente) for the many useful discussions. 


\section{References}

[1] M. P. Lutolf, J. A. Hubbell, Nat Biotech 2005, 23, 47

[2] J. Genzer, Annual Review of Materials Research 2012, 42, 435.

[3] S. T. Plummer, Q. Wang, P. W. Bohn, R. Stockton, M. A. Schwartz, Langmuir 2003, 19, 7528.

[4] R. R. Bhat, B. N. Chaney, J. Rowley, A. Liebmann-Vinson, J. Genzer, Advanced Materials 2005, 17, 2802.

[5] R. R. Bhat, M. R. Tomlinson, J. Genzer, Journal of Polymer Science Part B-Polymer Physics 2005, 43, 3384.

[6] L. Li, J. Wu, C. Gao, Colloids and Surfaces B-Biointerfaces 2011, 85, 12.

[7] J. A. Burdick, A. Khademhosseini, R. Langer, Langmuir 2004, 20, 5153.

[8] B. D. Polizzotti, B. D. Fairbanks, K. S. Anseth, Biomacromolecules 2008, 9, 1084.

[9] R. G. Wylie, S. Ahsan, Y. Aizawa, K. L. Maxwell, C. M. Morshead, M. S. Shoichet, Nat Mater 2011, 10, 799.

[10] A. Sala, P. Hanseler, A. Ranga, M. P. Lutolf, J. Vörös, M. Ehrbar, F. E. Weber, Integrative Biology 2011, 3, 1102.

[11] K. A. Mosiewicz, L. Kolb, A. J. van der Vlies, M. M. Martino, P. S. Lienemann, J. A. Hubbell, M. Ehrbar, M. P. Lutolf, Nat Mater 2013, 12, 1072.

[12] J. Shi, L. Wang, F. Zhang, H. Li, L. Lei, L. Liu, Y. Chen, ACS Applied Materials \& Interfaces 2010, 2, 1025.

[13] B. Zou, Y. Liu, X. Luo, F. Chen, X. Guo, X. Li, Acta Biomaterialia 2012, 8, 1576.

[14] Handarmin, G. Tan, B. Sundaray, G. Marcy, E. Goh, S. Chew, Drug Deliv. and Transl. Res. $2011,1,147$

[15] X. Zhang, X. Gao, L. Jiang, J. Qin, Langmuir 2012, 28, 10026.

[16] V. Milleret, B. R. Simona, P. S. Lienemann, J. Vörös, M. Ehrbar, Advanced Healthcare Materials 2014, 3, 508.

[17] S. A. DeLong, A. S. Gobin, J. L. West, Journal of Controlled Release 2005, 109, 139.

[18] S. A. DeLong, J. J. Moon, J. L. West, Biomaterials 2005, 26, 3227.

[19] D. Guarnieri, A. De Capua, M. Ventre, A. Borzacchiello, C. Pedone, D. Marasco, M. Ruvo, P. A. Netti, Acta Biomaterialia 2010, 6, 2532.

[20] X. Wang, E. Wenk, X. Zhang, L. Meinel, G. Vunjak-Novakovic, D. L. Kaplan, Journal of Controlled Release 2009, 134, 81.

[21] D. W. Hutmacher, Journal of Biomaterials Science-Polymer Edition 2001, 12, 107.

[22] S. F. Yang, K. F. Leong, Z. H. Du, C. K. Chua, Tissue Engineering 2002, 8, 1.

[23] W.-Y. Yeong, C.-K. Chua, K.-F. Leong, M. Chandrasekaran, Trends in Biotechnology 2004, 22, 643 .

[24] S. Edmondson, V. L. Osborne, W. T. S. Huck, Chemical Society Reviews 2004, 33, 14

[25] R. Barbey, L. Lavanant, D. Paripovic, N. Schuwer, C. Sugnaux, S. Tugulu, H. A. Klok, Chemical Reviews 2009, 109, 5437.

[26] S. Tugulu, A. Arnold, I. Sielaff, K. Johnsson, H.-A. Klok, Biomacromolecules 2005, 6, 1602.

[27] S. Tugulu, P. Silacci, N. Stergiopulos, H.-A. Klok, Biomaterials 2007, $28,2536$.

[28] H. Ma, J. Hyun, Z. Zhang, T. P. Beebe, A. Chilkoti, Advanced Functional Materials 2005, 15,

529.

[29] J. E. Raynor, T. A. Petrie, A. J. García, D. M. Collard, Advanced Materials 2007, 19, 1724.

[30] L. Moroni, M. Klein Gunnewiek, E. M. Benetti, Acta Biomaterialia 2014, 10, 2367.

[31] M. Kobayashi, Y. Terayama, H. Yamaguchi, M. Terada, D. Murakami, K. Ishihara, A. Takahara, Langmuir 2012, 28, 7212.

[32] G. Marletta, G. Ciapetti, C. Satriano, F. Perut, M. Salerno, N. Baldini, Biomaterials 2007, 28, 1132.

[33] S. Diamanti, S. Arifuzzaman, A. Elsen, J. Genzer, R. A. Vaia, Polymer 2008, 49, 3770.

[34] E. N. Chiang, R. Dong, C. K. Ober, B. A. Baird, Langmuir 2011, 27, 7016.

[35] M. Navarro, E. M. Benetti, S. Zapotoczny, J. A. Planell, G. J. Vancso, Langmuir 2008, 24 10996.

[36] 13039 .

S. Yuan, G. Xiong, X. Wang, S. Zhang, C. Choong, Journal of Materials Chemistry 2012, 22,

S. Yuan, G. Xiong, A. Roguin, C. Choong, Biointerphases 2012, 7.

T. Ren, Z. Mao, J. Guo, C. Gao, Langmuir 2013, 29, 6386. 


\section{Supporting Information}

\section{Average contact angle \\ $\left[^{\circ}\right]$}

PCL

ATRP initiator modified PCL

POEGMA modified PCL
$73 \pm 3$

$64 \pm 3$

$45 \pm 3$

Table S1. Contact angle values for pure and POEGMA-modified PCL spincoated films on silicon wafers.

a)

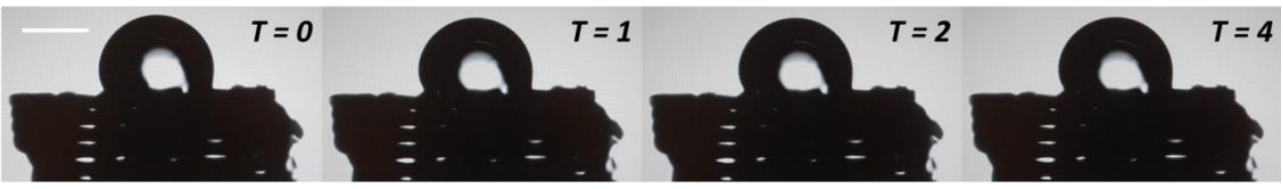

b)

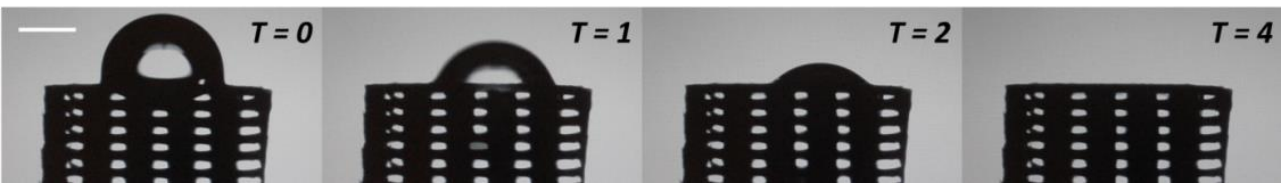

Figure S1. Snapshots taken at 1 and 2 seconds intervals of $4 \mu \mathrm{l}$ water drops placed on top of pure PCL (a) and POEGMA modified scaffolds (b). Scale bar is $1 \mathrm{~mm}$. 


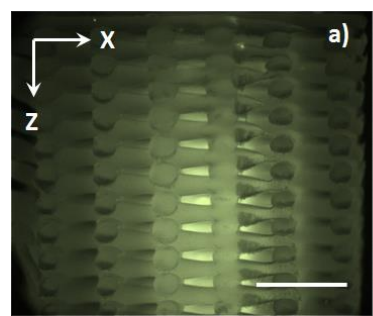

b)

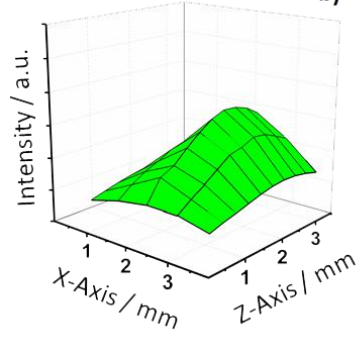

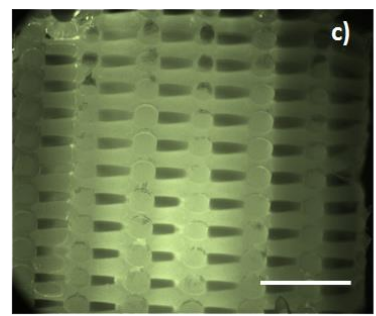

d) f)

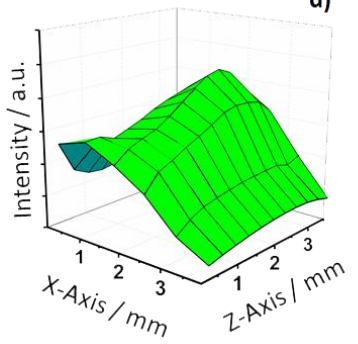

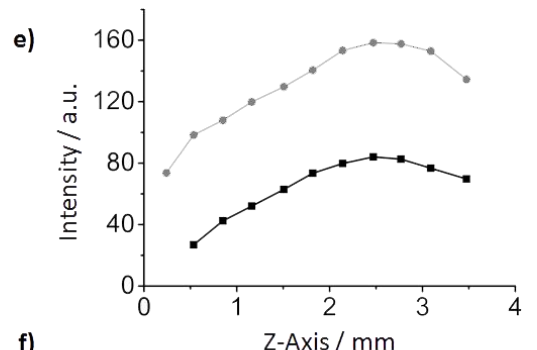

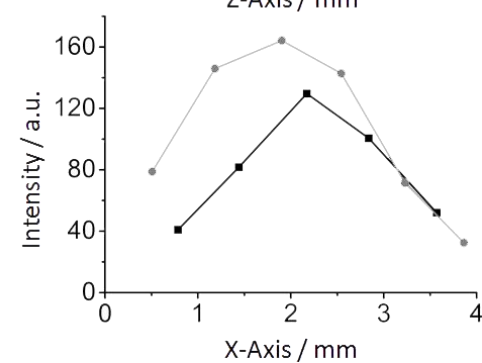

Figure S2. Fluorescent micrographs $(a, c)$ and the corresponding 3D intensity profile $(b, d)$ of the vertical cross-section of PCL-POEGMA scaffolds functionalized with BSA-FITC using 2 $\mu \mathrm{l}(\mathrm{a}, \mathrm{b})$, and $10 \mu \mathrm{l}(\mathrm{c}, \mathrm{d})$ microdroplets. Intensity profiles along the z-axis (e) and the x-axis (f) of the scaffold: $\mathbf{\square}) 2 \mu \mathrm{l}$, and •) $10 \mu \mathrm{l}$. Scale bar is $1 \mathrm{~mm}$.
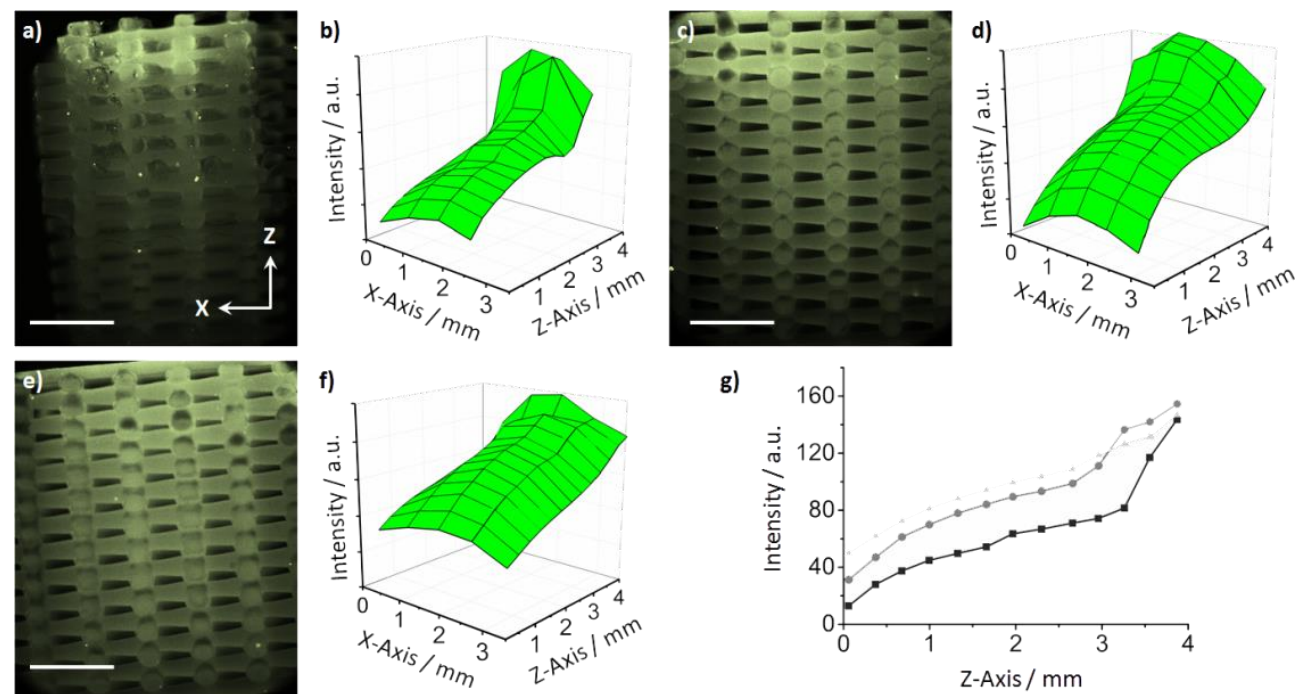

Figure S3. Fluorescent micrographs $(\mathrm{a}, \mathrm{c}, \mathrm{e})$ and the corresponding $3 \mathrm{D}$ intensity profiles $(b, d, f)$ from the vertical cross-section of PCL-POEGMA scaffolds functionalized with BSAFITC after $30 \mathrm{~min}(\mathrm{a}, \mathrm{b}), 60 \mathrm{~min}(\mathrm{c}, \mathrm{d})$, and $120 \mathrm{~min}(\mathrm{e}, \mathrm{f})$ of diffusion/functionalization times. Intensity profiles along the y-axis of the scaffolds (g) following: -) $30 \mathrm{~min}$., •) $60 \mathrm{~min}$. , and ) $120 \mathrm{~min}$ of diffusion/functionalization times. Scale bar is $1 \mathrm{~mm}$. 

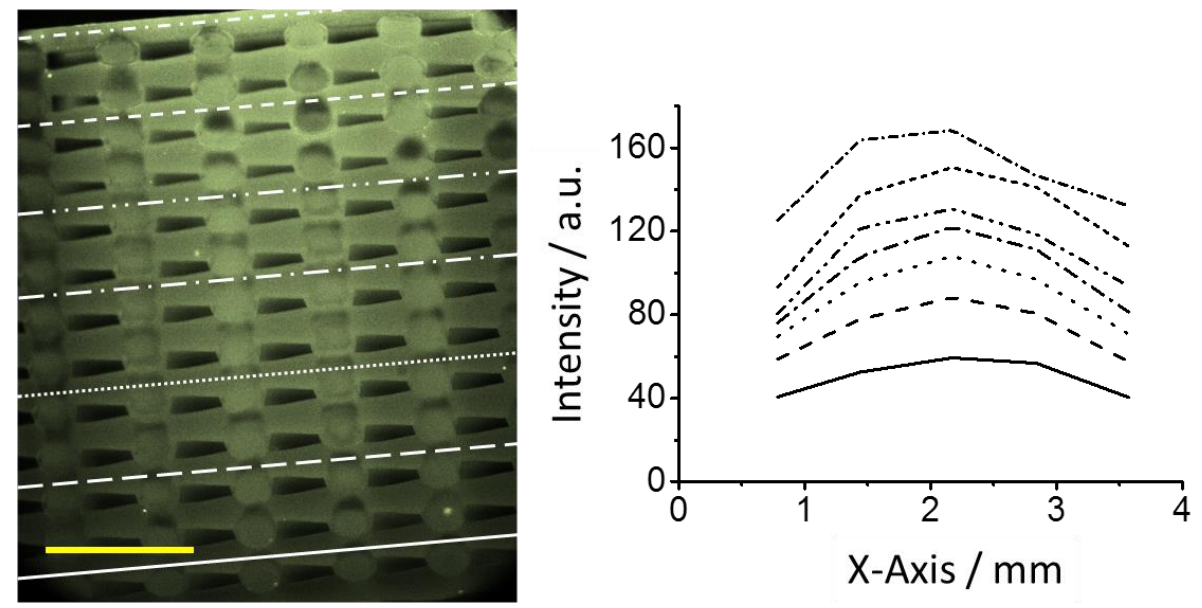

Figure S4. Fluorescent micrograph and the corresponding $2 \mathrm{D}$ intensity profile along the $\mathrm{x}$ axis at different positions along the z-axis from the vertical cross-section of a PCL-POEGMA scaffold functionalized with BSA-FITC. Scale bar is $1 \mathrm{~mm}$.

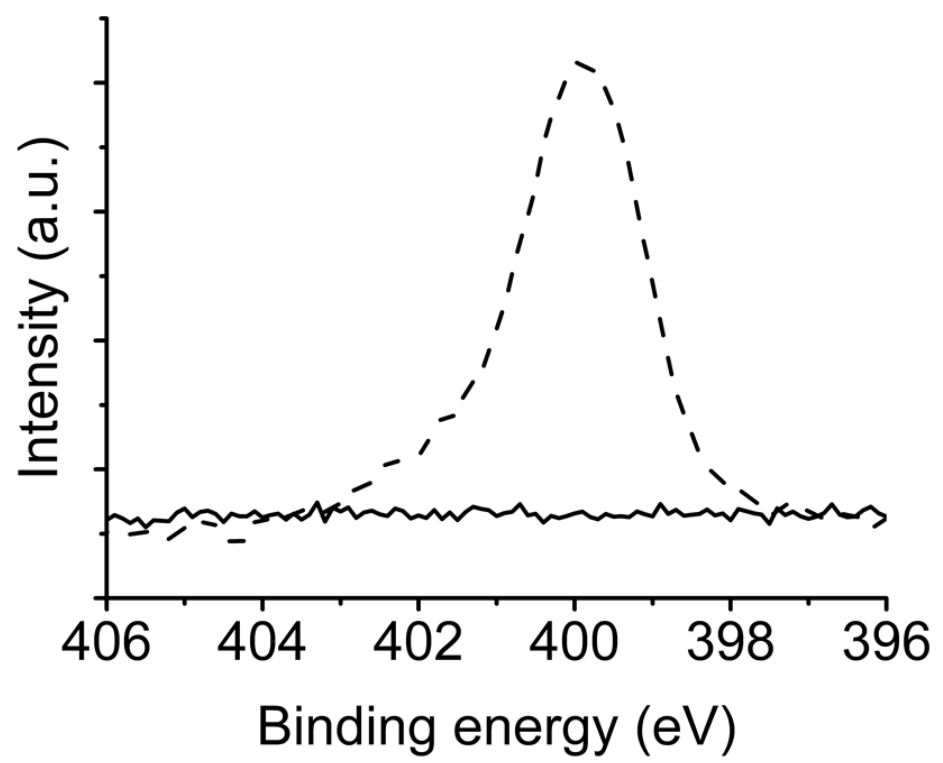

Figure S5. Averaged N1s spectra measured by XPS on unfunctionalized (solid line) and FN functionalized (dashed line) POEGMA covered PCL. 

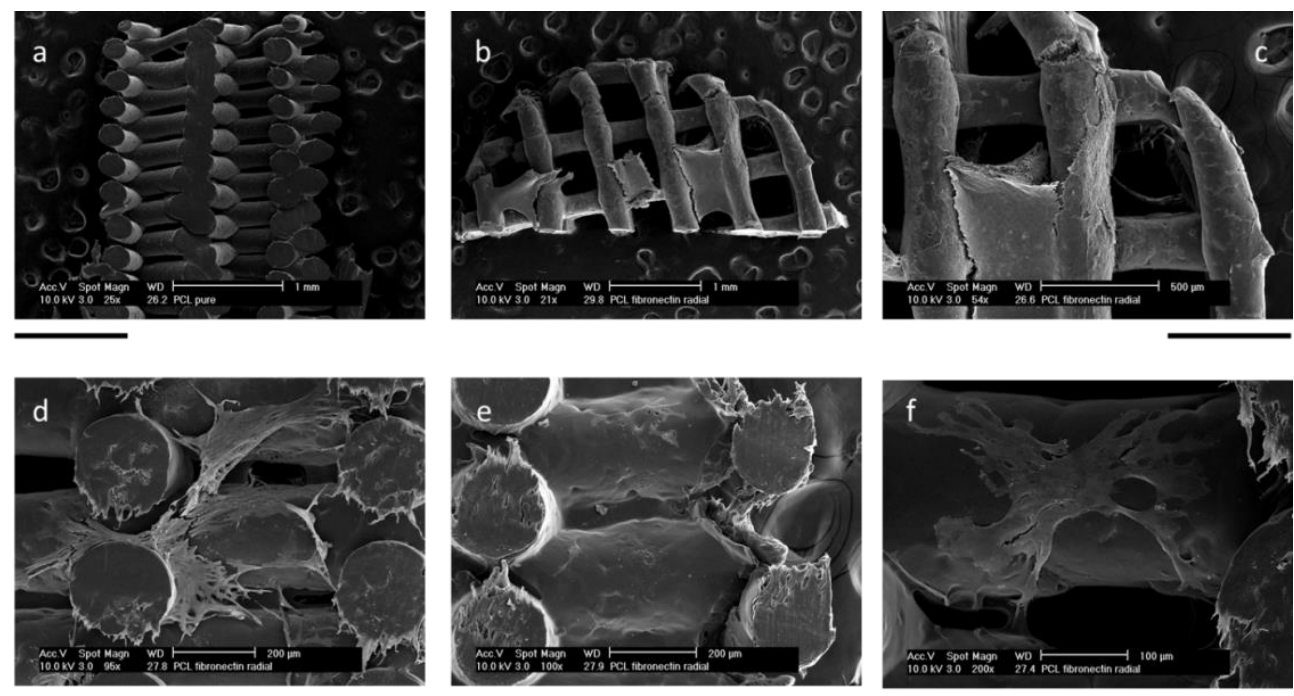

Figure S6. Scanning Electron Microscopy (SEM) images of a) non functionalized PCL scaffolds without cells and $b-f)$ representative images of the appearance of the hMSCs and their ECM seeded onto fibronectin-functionalized scaffolds. Scale bars: a,b) $1 \mathrm{~mm}$; ) $500 \mu \mathrm{m}$; d,e) $200 \mu \mathrm{m}$; f) $100 \mu \mathrm{m}$.

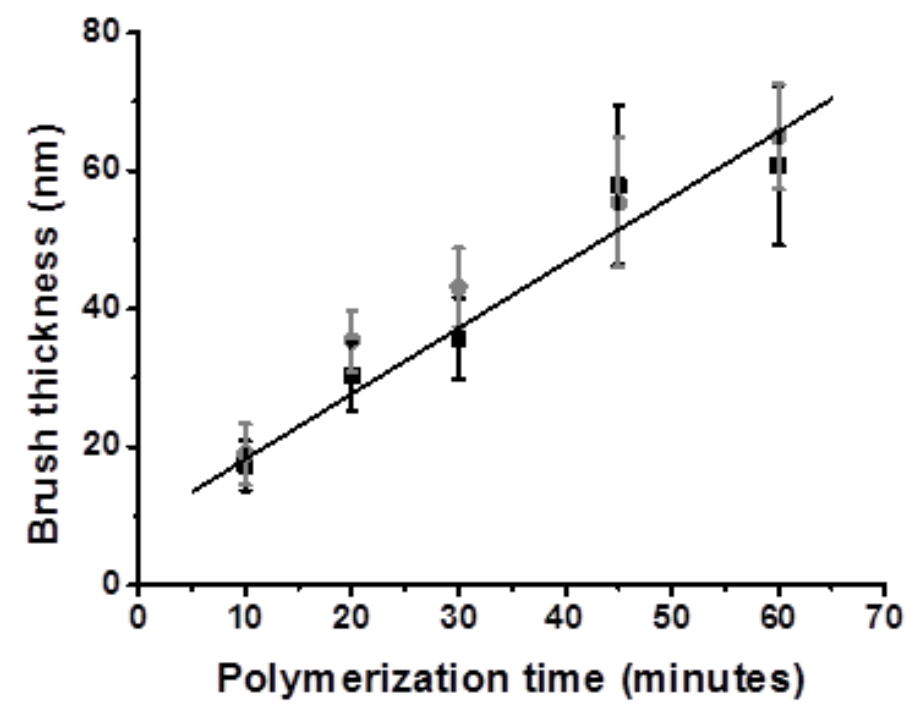

Figure S7. Dry thickness of POEGMA brushes as a function of polymerization time measured by ellipsometry. 


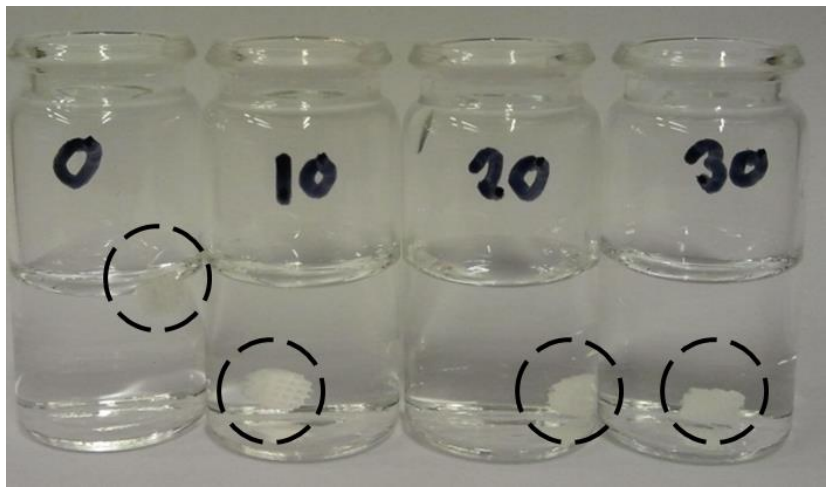

Figure S8. Photograph of PCL scaffolds modified with 0 (pure PCL), 10, 20, and $30 \mathrm{~nm}$ thick POEGMA brushes. Pure PCL scaffolds are floating and POEGMA modified scaffolds are at the bottom of a water-filled vial. 


\section{CHAPTER 8}

\section{Binding growth factors to 3D plotted scaffolds: are polymer brush-growth factor gradients an option for osteochondral tissue regeneration?}

Andrea Di Luca*, Michel Klein-Gunnewiek*, Julius Vancso, Clemens van

Blitterswijk, Edmondo Benetti ${ }^{+}$, Lorenzo Moroni, ${ }^{+}$.

† These authors contributed equally to this work

* These authors contributed equally to this work. 


\section{Abstract}

Engineering the osteochondral tissue presents some challenges mainly relying in its function of transition from the subchondral bone to the cartilage and the gradual variation in several biological, mechanical and structural features. Cell number, water content, and collagen type II decrease from the articular surface to the deep zone, whereas glycosaminoglycans (GAG) and cell size present the opposite trend. Consequently, stiffness increases when moving toward the deep zone. Collagen fiber disposition also changes when moving from the articular surface to the deep zone: they are completely parallel in the articular surface and become perpendicular in the deep zone of articular cartilage.

A possible solution for osteochondral regeneration, therefore, might be the design and fabrication of scaffolds presenting a gradient able to mimic this transition. Covalent binding of biological factors, in particular, has proven to enhance cell adhesion and differentiation in two-dimensional culture substrates. In the present work, we used polymer brushes as selective linkers of bone morphogenetic protein-2 (BMP-2) and transforming growth factor- $\beta 3$ (TGF- $\beta 3$ ) on the surface of $3 D$ scaffolds fabricated via additive manufacturing (AM). These growth factors (GFs) are known to stimulate the differentiation of human mesenchymal stromal cells (hMSCs) toward the osteogenic and chondrogenic lineages, respectively. BMP-2 and TGF- $\beta 3$ were covalently bound both homogeneously within a poly(ethylene glycol) (PEG)-based brush-functionalized scaffolds, and following a gradient composition by varying their concentration along the axial section of the 3D constructs. Opposite brush-supported gradients of BMP-2 and TGF- $\beta 3$ were finally generated and subsequently tested to stimulate hMSCs to differentiate towards an osteochondral-like state. The brush-supported GFs significantly influenced hMSC osteochondral differentiation when the scaffolds were homogenously modified, yet no effect was observed in the gradient scaffolds. Therefore, this technique seems promising to maintain the biological activity of growth factors covalently linked to 3D scaffolds, but needs to be further optimized in case biological gradients are desired. 


\section{Introduction}

In the last decades regenerative medicine relied on the use of 3D structures, namely scaffolds, to support cell adhesion and tissue growth. Several studies provided a number of techniques to generate scaffolds, such as gas foaming [1], salt leaching [2], freeze drying [3] or solvent casting [4]. All these techniques present well known limitations, such as the lack of pore interconnectivity, the impossibility to fine tune the pore size and the geometry of the constructs. In alternative to these well-established fabrications, additive manufacturing (AM) has progressively allowed to generate scaffolds with a fully interconnected pore size, a desired structural design, and it has enabled the possibility to fine tune scaffold properties during its production. Additionally, whereas it is possible to functionalize any scaffold with biological factors to improve cell attachment and differentiation, AM scaffolds offer the appealing advantage to create gradients thanks to the capillary forces originated in the pore network [5]. Growth factors such as bone morphogenetic protein (BMP) and transforming growth factor $\beta$ (TGF $\beta$ ) are known to trigger the differentiation of human mesenchymal stromal cells (hMSCs) toward the osteogenic or chondrogenic lineage [6-9]. A number of studies applied scaffolds in combinations with growth factors (GFs) [10-14] to improve hMSC differentiation. These techniques are highly expensive, due to the great dosage of GFs used [15], which can also lead to adverse effects [16, 17]. On the other hand, linking GFs to the surface ensures a continuous mono-dose treatment, which may also continuously impinge on the underlying signaling pathways, instead of continued infusion of GFs during the culture time.

GFs in combination with hMSCs were applied in the attempt to regenerate the osteochondral tissue. The osteochondral interface can be seen as a gradient tissue allowing the transition from the mineralized bone to the soft and highly hydrated cartilage [18]. Within the osteochondral tissue, gradual variations of growth factors, cell number, collagen orientation and mineralization are present. Due to this complex variation in structure, mechanical properties and biological components, the regeneration of this interface remains an open challenge in the field of tissue regeneration. The degeneration of osteochondral tissue progresses with age. 
Therefore, the need of scaffolds and techniques to support an effective regeneration will increase in the following years with the increase of life expectancy. In order to mimic the progressive variation of chemical characteristics of the osteochondral extra-cellular matrix (ECM), we propose here a scaffold fabrication based on AM of Polycaprolactone (PCL), to create fully interconnected $3 \mathrm{D}$ supports, later on modified with poly[oligo(ethylene glycol)methacrylate] (POEGMA) brushes applying surface-initiated atom transfer radical polymerization (SI-ATRP) from initiator functions on the scaffold surface. The functionalizable POEGMA brushes on the scaffolds were subsequently functionalized with BMP-2 and TGF- $\beta 3$. The constant presence of BMP-2 or TGF- $\beta 3$ should determine a continuous stimulus for the hMSCs to differentiate, as we hypothesized that the underlying biological signaling pathways would be continuously activated. We also linked the BMP-2 and TGF- $\beta 3$ in a double gradient fashion in order to stimulate the differentiation of hMSCs within the construct towards an osteochondral like tissue interface in vitro. The GF homogenously linked to the surface of the 3D scaffolds outperformed the simple addition of their soluble form to the cell culture media, determining the overexpression of osteogenic and chondrogenic markers in the shorter time span of 10 days when normally the expression takes between 3 and 4 weeks with the soluble factors. 


\section{Results and discussion}

a

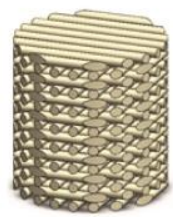

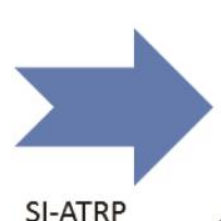

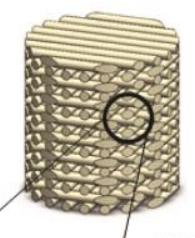

BMP-2/TGF- $\beta 3$ solution
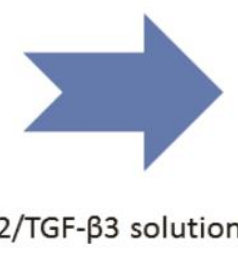
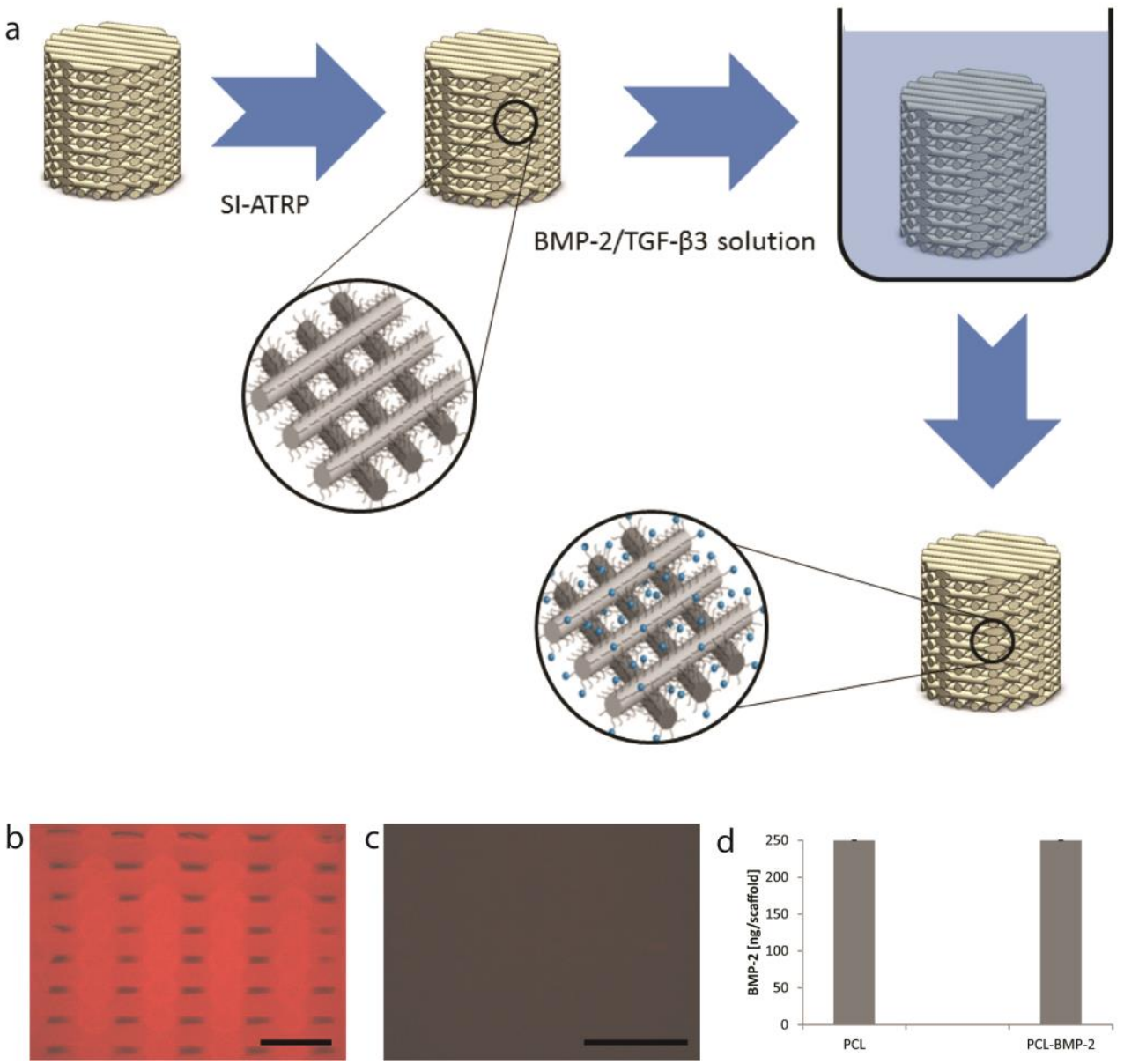

Figure 8. Cartoon summarizing the functionalization process (a), fluorescent micrographs of BMP-2 fully functionalized and bare PCL scaffolds $(b, c)$ and plot showing the BMP2 amount adsorbed on PCL scaffolds and linked to the PCL-POEGMA brushes before the washing steps after the functionalization (d). Scale bar $650 \mu \mathrm{m}$ (b) and $1 \mathrm{~mm}$ (c).

Figure 1 depicts the general strategy of the proposed fabrication. The scaffold was plotted using an extrusion-based additive manufacturing system (Bioscaffolder, SysENG, Germany). Briefly, the polymer was placed in a stainless syringe and processed at $100^{\circ} \mathrm{C}$. The molten polymer was extruded through a cartridge unit by the application of a nitrogen flow with a pressure of 5 bar from a pressurized cap 
following a computer aided design/computer aided manufacturing (CAD/CAM) program. After plotting, PCL scaffolds were fully functionalized with POEGMA chains, as early described by our group [5]. Briefly, scaffolds were modified by surface-initiated atom transfer radical polymerization (SI-ATRP) of OEGMA generating POEGMA brushes presenting an average dry thickness of around 10 $\mathrm{nm}$. POEGMA brushes uniformly covering the initiator-functionalized PCL 3D scaffolds were obtained by immersing the supports in the polymerization medium for 10 minutes (See Experimental Section for Details). Following extensive rinsing, to remove unbound species and residual catalyst, the POEGMA brush-3D scaffolds were uniformly functionalized with BMP-2 and TGF- $\beta 3$ (finally yielding BMP-2-POEGMA brush and TGF- $\beta$-POEGMA-brush 3D scaffolds) (Figure 1a). The choice of POEGMA as linker is related to its antifouling activity. In order to let the GFs provide a constant differentiating stimulus to the cells, we designed the functionalized scaffolds in a way they can only adhere on the portions in which the GFs are presented (Fig 2a-b and 4c-d).

BMPs belong to the so-called TGF- $\beta$ superfamily which shares a peculiar folding: TGF- $\beta 2$ crystal analysis showed that the monomer lacks a well-defined hydrophobic core and displays an unusual elongated non-globular fold. Eight cysteines form four intrachain disulfide bonds, which are clustered in a core region forming a network complementary to the network of hydrogen bonds. The dimer is stabilized by the ninth cysteine, which forms an interchain disulfide bond, and by two identical hydrophobic interfaces. Via sequence profile has been shown that other members of the TGF- $\beta$ superfamily also adopt this unusual "TGF- $\beta$ fold" [19]. The amount of GFs attached on the PCL scaffolds and POEGMA brush-PCL scaffolds was quantified via ELISA on the supernatant solution used for the functionalization reaction. Being part of the same superfamily, BMP-2 (Fig 1d) and TGF- $\beta 3$ (Fig S1) displayed similar attachment, with a $99.94 \pm 0.02 \%$ and a $99.67 \pm$ $0.04 \%$ coupling efficiency for BMP-2 and TGF- $\beta 3$ respectively. Despite a similar attachment could be measured on bare PCL scaffolds as well as on POEGMA brush-PCL scaffolds, it is to be noted that in the first case GFs just adsorbed on the scaffolds, whereas in the second the GFs were covalent bound to the brushes. To confirm the presence of the active growth factor on the surface, an antibody 
staining of the binding site of BMP-2 (Fig 1b, c) was performed. The functionalized scaffolds showed a uniform presence of the GF binding site, in contrast to the nonfunctionalized scaffolds (Fig 1d and S1). Whereas the adsorbed growth factors are washed away during sterilization and culture medium immersion prior to cell seeding, the growth factors linked to the nanobrushes, being covalently bound, remain attached to the scaffolds. This is one of the advantages of using a brush "spacer" for covalently bound GFs to a 3D scaffold. The GFs are available at the interface with the adhering cells. In addition, they are robustly linked to the support, in contrast to physisorbed proteins on similar, yet bare, polyester constructs.
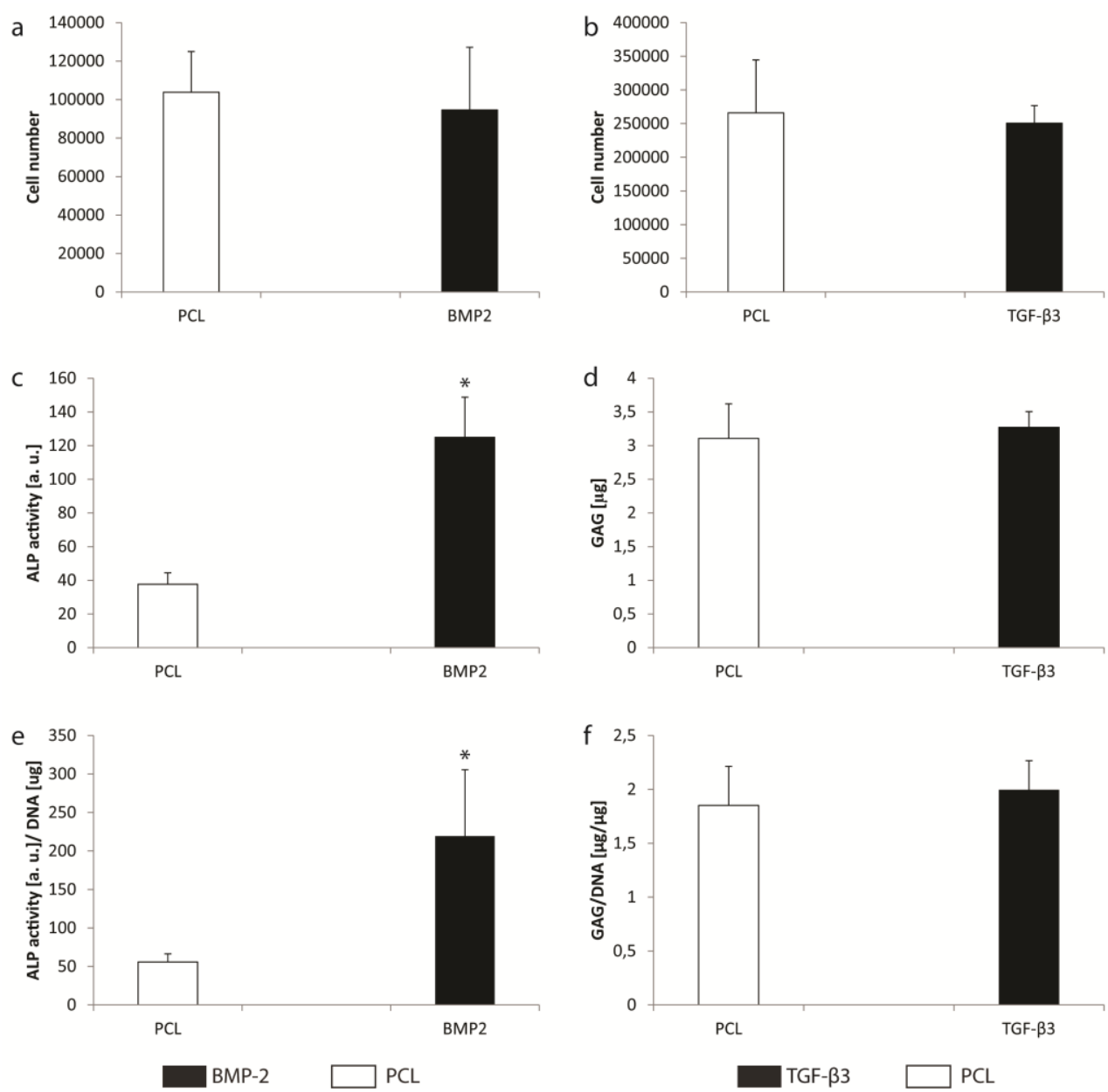

Figure 2. Graphs displaying the cell number on PCL scaffolds and scaffolds functionalized with BMP2 and TGF- $\beta 3$ (a, b). ALP activity and ALP activity normalized by DNA of BMP-2 
functionalized scaffolds with respect to PCL scaffolds. (c, e) Glycosaminoglycans (GAGs) amount and GAG normalized by DNA of TGF- $\beta 3$ functionalized scaffolds with respect to PCL scaffolds $(d, f) .{ }^{*}$ statistical significance $p<0.05$

To further confirm the activity of the growth factors bound to the surface, an ALP and GAG analysis was conducted on PCL scaffolds seeded with hMSCs and cultured for 10 days in basic medium (Fig 2). As a negative control, POEGMA brush-PCL scaffolds did not show cells attached, due to their antifouling properties. Therefore, no ALP activity, cell number or GAG amount could be measured (data not shown).

As expected, scaffolds presenting POEGMA brush layers decorated with either BMP-2 or TGF- $\beta 3$ stimulated the adhesion of hMSCs, confirming the already reported activity of these GFs as not only differentiation but also cell adhesive proteins [20, 21]. The ALP activity was significantly higher both on a structural (per scaffold, Fig. 2c) and on a cellular (per DNA, Fig. 2e) level on functionalized PCL scaffolds with respect to bare PCL scaffolds. On the other hand, hMSCs cultured on TGF- $\beta 3$ functionalized scaffolds didn't show any difference in GAG synthesis, both at a structural as well as at a cellular level with respect to hMSCs cultured on PCL scaffolds (Fig. 2d, f). Since the functionalized and non-functionalized scaffolds presented the same cell number, the normalization by DNA amount did not determined any variation on the significance of the differences within the data groups. 

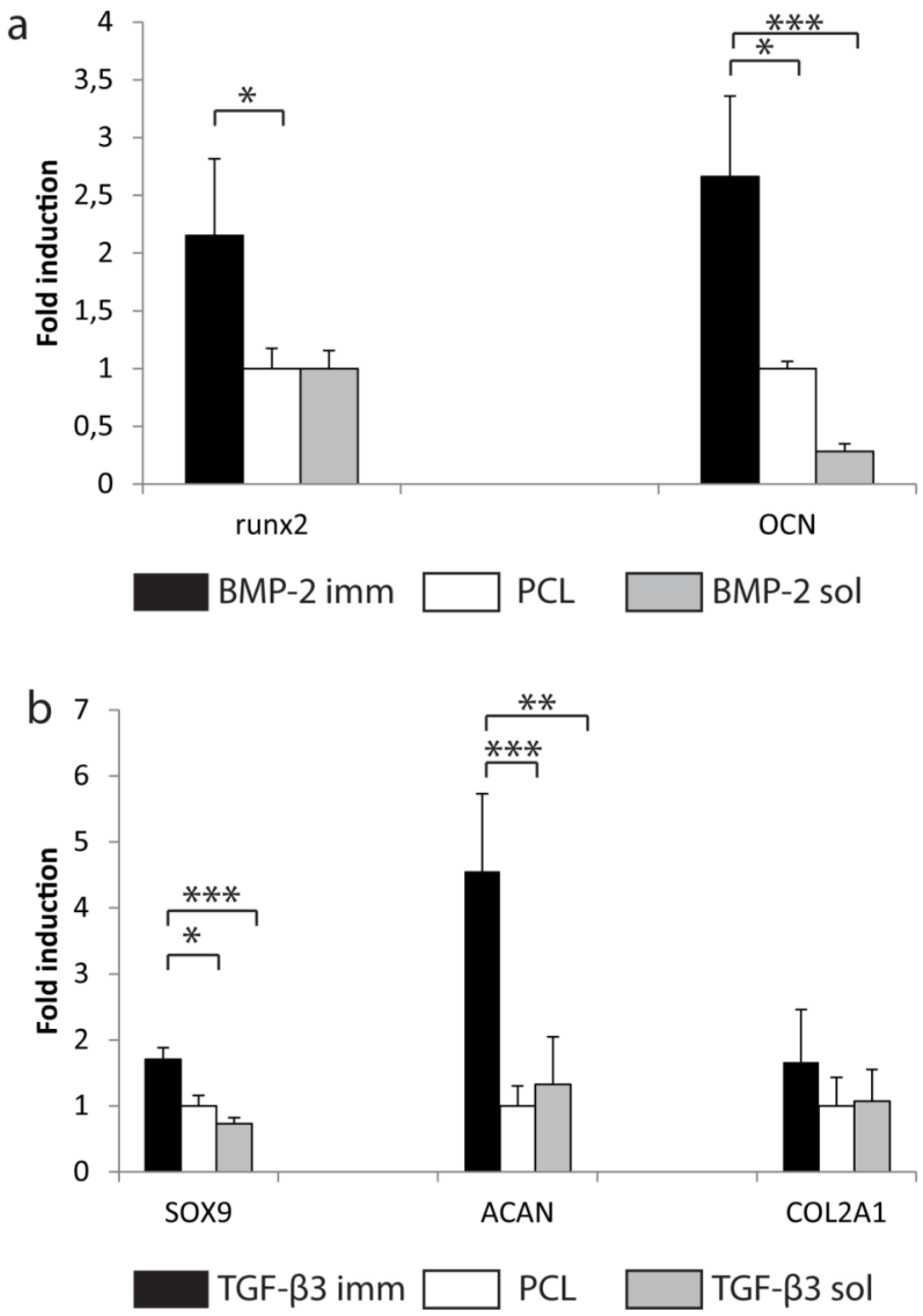

Figure 3. Plots showing the fold induction of osteogenic (runx2, OCN) and chondrogenic (sox9, ACAN, coll2a1) genes in hMSCs cultured on scaffolds functionalized with BMP-2 and TGF- $\beta 3$ with respect to hMSCs cultured on PCL scaffolds and PCL scaffolds with the cell culture media supplemented with the growth factor (c, d). * statistical significance $p<0.05$, ${ }^{* *} p<0.01,{ }^{* * *} p<0.005$ 
To further test the activity of the GF-POEGMA brush-PCL scaffold, the expression of selected osteogenic and chondrogenic markers was assessed on the functionalized scaffolds, comparing bare PCL scaffolds and PCL scaffolds cultured in basic medium supplemented with the same GFs dispersed in the medium (not bound to the scaffold's surface) (Fig. 3). Both GFs linked to the scaffolds surface were not only active, but outperformed the addition of their soluble form to the cell culture media. Runt-related transcription factor (Runx2) and osteocalcin (OCN) were significantly upregulated in scaffolds presenting BMP-2 on the surface compared to both the PCL scaffolds cultured in basic medium. Culturing hMSCs on PCL scaffolds in basic medium supplemented with soluble BMP-2 did not increase the expression of the early osteogenic marker runx2 and caused the downregulation of the late osteogenic marker OCN (Fig 3a). In literature, the effect of BMP-2 addition to cell culture media was reported and was very controversial. Some studies indicated that continuous stimulation with BMP2 $(0.1-800 \mathrm{ng} / \mathrm{mL})$ affects the differentiation but not the proliferation of hMSCs [22, 23], whereas other studies did not demonstrate an osteogenic effect of $100 \mathrm{ng} / \mathrm{mL}$ BMP2 [24, 25]. The data presented in literature are supported by our results. In particular Runx2 is an essential regulator of hMSC differentiation into the osteogenic lineage [26]. In vitro studies indicated that Runx2 triggers the expression of major bone protein genes like collagen type I, osteopontin, bone sialoprotein and osteocalcin during the early stage of osteoblast differentiation [27]. As expected, since runx2 was not upregulated, also OCN didn't show any upregulation when BMP-2 was supplemented in culture medium. Yet, the use of immobilized BMP-2 resulted in early osteogenic differentiation, thus possibly indicating a more powerful route towards designing bioactive scaffolds to guide stem cell activity. Sox-9 and aggrecan levels were significantly higher on TGF- 33 -POEGMA brush-PCL scaffolds compared to the PCL scaffolds cultured in basic medium. The basic medium supplemented with soluble TGF- $\beta 3$ caused a downregulation of the early marker sox-9 and didn't increase the expression of aggrecan. The late marker collagen type 2 was not changed by the functionalization with TGF- $\beta 3$ nor by the culture in medium supplemented with the growth factor (Fig 3b). 
Cells in direct contact with the functionalized surface presenting the GFs were constantly stimulated to differentiate. Soluble factors dispersed in solution were not as effective as the linked proteins, which determined higher concentration compared to the whole amount of soluble GFs used during the culture time, in the area where the cells were attached. Additionally, the half-life of soluble BMP-2 and TGF- $\beta 3$ is much shorter than the time of replenishing the culture medium. Conversely, the biological molecules linked to the surface determines a shorter differentiation time of 10 days due to the constant stimulus, whereas the differentiation of hMSCs treated with soluble factors occurs after 3 to 4 weeks at least [28]. Linking growth factors to the surface reduces the costs with a monodose treatment which prevents the continued infusion of expensive growth factors during the culture time. hMSC differentiation using growth factors such as BMP-2 and TGF- $\beta 3$ is widely described in $2 \mathrm{D}$ in vitro. The main limitation of $2 \mathrm{D}$ supplementation is the constant infusion of the growth factors at concentration of 5$20 \mathrm{ng} / \mathrm{mL}$ in the culture media [25, 29, 30]. The use of growth factor supplemented cell culture media must be ensured for the whole culture time in order to prevent de-differentiation of hMSCs [31, 32]. A few studies described the use of growth factors linked to materials' surface. The major disadvantage of these studies, however, is the use of highly concentrated growth factor solutions. For techniques such as inkjet printing, the preparation of the ink was based on $10 \mu \mathrm{g} / \mathrm{mL}$ GFsolution, as described by Miller et al [33], whereas Cabanas-Danés et al. used a BMP-6 solution of $20 \mu \mathrm{g} / \mathrm{mL}$ [34], which is 8 times higher than the one described in this paper.

To further exploit the potential of covalently bound GFs, brush gradients were built in the 3D scaffolds as previously shown [5]. Briefly, GF gradients along the $z$ axis (Figure 4a, b), were obtained by applying a porous paper sheet as solution reservoir and allowing the protein medium to diffuse by capillary forces into the functionalizable POEGMA brush-PCL scaffolds. The lower fibers, being in contact for longer time with the GF, presented a higher amount of the linked BMP-2 and TGF- $\beta 3$, which decreased along the $z$ axis. Additionally, scaffolds presenting a double gradient were produced (Fig $4 \mathrm{a}$ ). The same strategy used to produce a 
single gradient was followed; as additional step, scaffolds were placed up-side down in contact with the porous paper sheet soaked with the second GF solution. The effect of the BMP-2 and TGF- $\beta 3$ gradient on the hMSCs residing in the different area of the scaffolds was determined again after 10 days in basic medium. Since the POEGMA brushes are anti-fouling, POEGMA brush-PCL scaffold displayed no cell attachment. PCL scaffolds as well as GF-POEGMA brush-PCL scaffold displayed the same cell number due to the activity of BMP-2 and TGF- $\beta 3$ as adhesion proteins. Scaffolds presenting gradients of BMP-2 and TGF- $\beta 3$ showed a significantly higher number of cells in the portion of the scaffold with the higher concentration of GF with respect to the other half. This confirmed the presence of a gradient along the $z$ axis. In the bottom part of the scaffolds, the concentration of GFs was higher, determining a greater number of anchoring point for the hMSCs. On the top half, the major part of the surface was covered only by the antifouling brushes and cells had less adhesion sites available (Fig 4a-d).

Although full scaffolds displayed a significantly higher ALP activity, no major differences could be seen between the bottom and top part of the gradient scaffolds (Fig 4e). At a cellular level, when the ALP was normalized by DNA no differences could be seen among the PCL scaffolds, the BMP-2-POEGMA brushPCL scaffolds, and the top part of the gradient scaffolds. However, a significantly higher ALP activity was measured on the top part with respect to the bottom part, which might be due to the very low cell number. In order to further evaluate hMSC osteogenic differentiation, $G$ scaffolds were cut into 3 parts (bottom, mid and top) and osteogenic markers were analyzed via PCR (Fig S2). No major differences were seen between the different areas. 
a

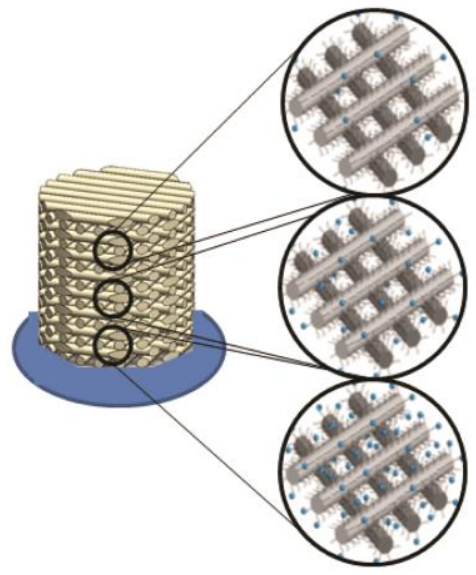

C

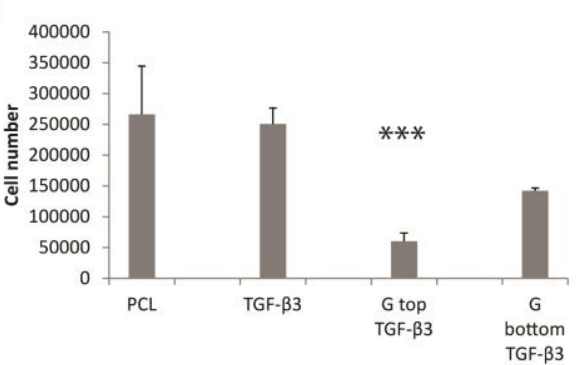

e
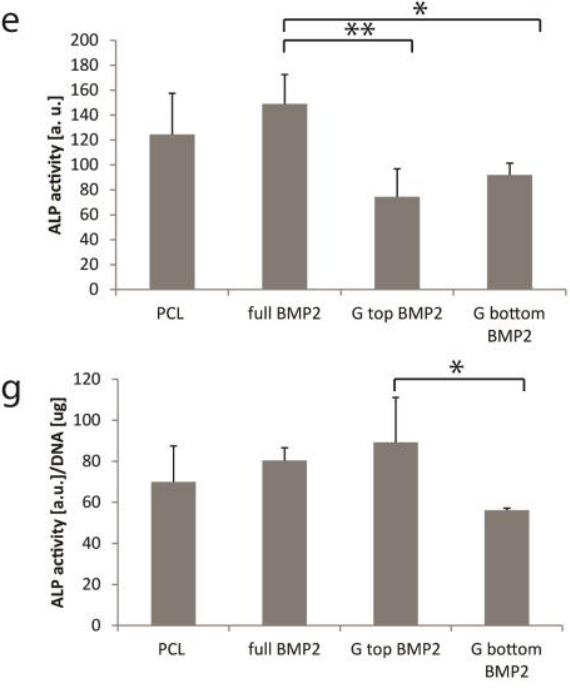

b

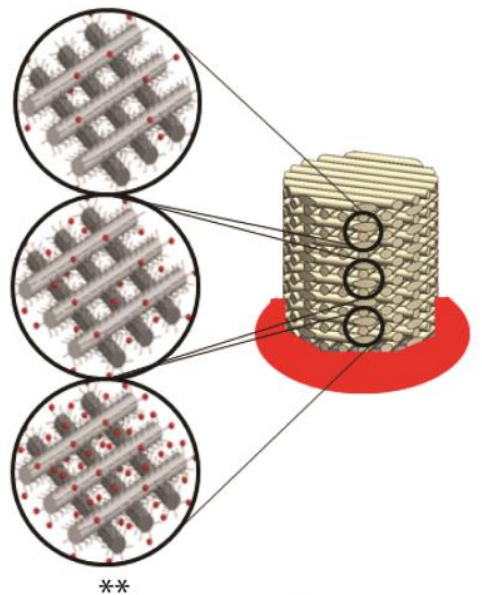

d

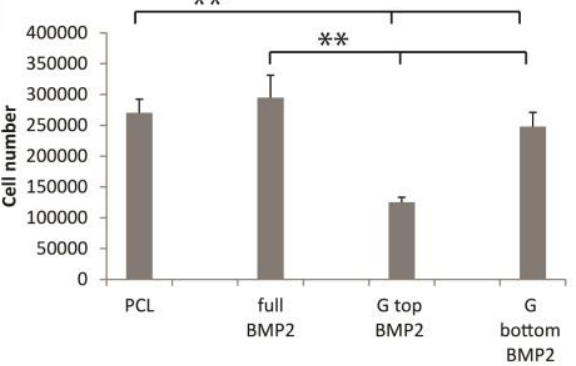

$f$

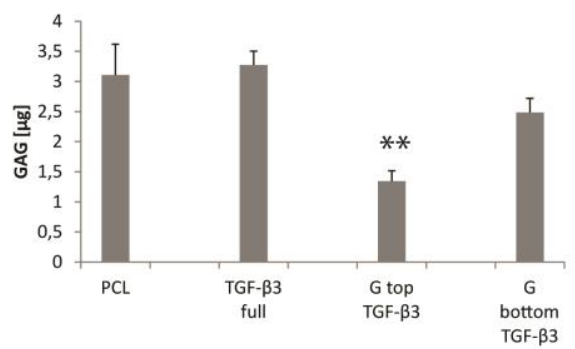

$\mathrm{h}$

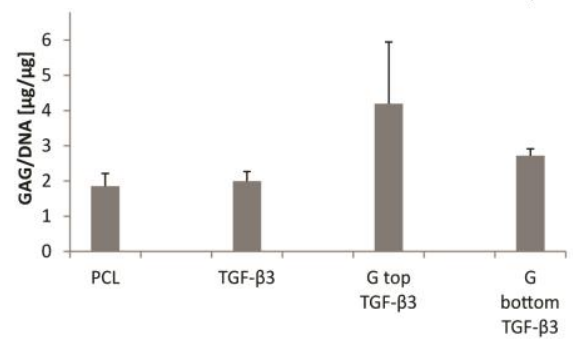

Figure 4. Cartoon displaying the gradient functionalization with BMP-2 (a) and TGF- $\beta 3$ (b). Plots displaying cell attachment in fully functionalized scaffolds, PCL scaffolds and bottom and top part of the gradient (c, d); G top BMP2 showed a significantly lower cell number with respect to all the other conditions (c). ALP expression (e) and ALP activity normalized by 
DNA amount $(\mathrm{g})$ of hMSCs cultured on BMP-2 fully functionalized scaffolds, gradient scaffolds and PCL scaffolds. Plots showing GAG per scaffold (f) and normalized by DNA (h) of hMSCs cultured on PCL scaffolds, fully TGF- $\beta 3$ functionalized scaffolds and top and bottom part of the gradient. ${ }^{*}$ Statistical significance $p<0.05,{ }^{* *} p<0.01,{ }^{* * *} p<0.005$

The same analysis was performed on TGF- $\beta 3$ functionalized scaffolds. To assess chondrogenic differentiation, GAG analysis was performed. At a cellular level, the trend was similar to the one displayed by ALP activity: no statistically significant differences were displayed between functionalized and non-functionalized scaffolds and within the functionalized scaffolds between the top and the bottom part of the scaffolds. As previously explained, this might be due to the low cell number present in the top part of the gradient and to the presence of anti-fouling brushes. When considering the total GAG per scaffold, a statistically significant difference in GAG production was measured in the lower and top part of the scaffolds. Yet, no differences were observed between the bare PCL and fully functionalized scaffolds, indicating that further studies should aim at longer culturing time. The lack of chondrogenic differentiation might also be due to the culture for 10 days in basic medium. Bilgen et al. demonstrated that there is an interaction between the FBS, contained in the basic medium, and the TGF- $\beta 3$ which can interfere with its activity [35]. 

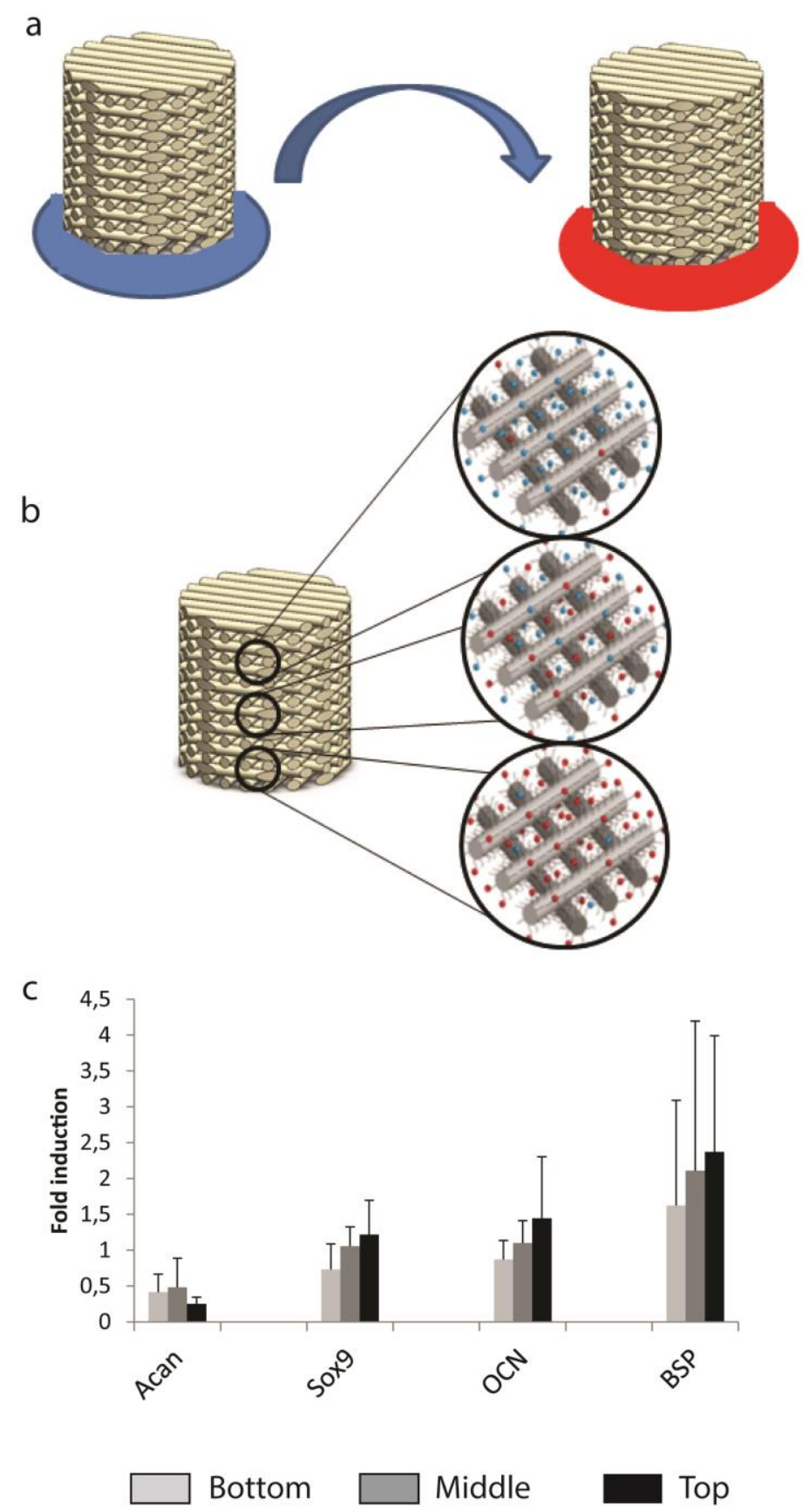

Figure 5. Cartoon displaying the double functionalization in a gradient fashion with BMP-2 and TGF- $\beta 3$ (a) and the resulting disposition of the growth factors within the scaffold (b). 
Fold induction of chondrogenic markers (aggrecan, sox9) and osteogenic markers (osteocalcin, bone sialoprotein) in the different portion of the scaffolds.

To limit the differences in cell number and to determine if the combined effect of the 2 GFs could have triggered the expression of osteogenic and chondrogenic genetic markers, a double gradient was generated (Fig 5a, b). After 10 days in basic medium, scaffolds were divided into 3 parts: bottom, mid and top (Fig 5c). Neither the chondrogenic markers nor the osteogenic ones were upregulated in any of the scaffolds' portion.

In this study, we presented a platform based on a continuous stimulation of cells through covalent binding of growth factors on the surface of 3D plotted scaffolds. Cell differentiation was enhanced on the fully functionalized scaffolds compared to conventional supplementation of soluble growth factors in the culture medium. This was probably due to a sufficient presence and concentration of growth factors throughout the whole surface. hMSCs adhering on the scaffolds were immediately stimulated in the BMP-2 driven osteogenic differentiation and TGF- $\beta 3$ driven chondrogenic differentiation. We might speculate that cells in direct contact with the scaffold backbone, while differentiating, released bioactive molecules that regenerated within the pore a differentiating microenvironment, which stimulated cells not directly adhering to the scaffolds to start the osteogenic or chondrogenic differentiation process.

Despite we have shown that covalently bound GFs could successfully triggered hMSC differentiation when homogeneously decorating 3D plotted scaffolds, the gradient constructs did not show any sign of cell differentiation. In the gradient structure the overall concentration of growth factors was significantly lower and probably not sufficient to trigger a cell-cell propagation of the differentiation process. Several studies in literature presented scaffolds functionalized with growth factors as release platforms [12, 36-39]. The concept of growth factor delivery presents several disadvantages, such as the need of scaffolds with high loading efficiency and a fine controlled release kinetic in order to reach the working concentration at the target site. Additionally, once released the growth factor has a short half-life in vivo [40], thus needing a carrier or protector. Nanobrushes alongside being the linker chain between the surface of the scaffold and the growth 
factor provides protection and ensure its bio-activity. As shown in figure 2, the BMP-2 and TGF- $\beta 3$ functionalized scaffolds determined the upregulation of osteogenic and chondrogenic markers respectively, whereas the soluble factors brought to a downregulation of the same genes. This lack of activity can be related to a short half-life in vitro. Although release platforms can be designed to deliver a signal in a time specific and sequential manner [38, 41], they lack the ability to fine tune the presentation of the growth factors in a specific area of the scaffold. In osteochondral regeneration the position of BMP-2 and TGF- $\beta 3$ within the scaffold is fundamental for providing the osteogenic stimulus in the part of the scaffold in contact with the subchondral bone, decreasing toward the joint surface, while the chondrogenic signal increasing in the same direction. The method presented in this work showed to be more effective and cheaper than the addition of soluble growth factors in solution for homogenously functionalized scaffolds. Further studies are needed to understand if such promising strategy is also beneficial in single and double gradient scaffolds.

\section{Materials and methods}

\section{Scaffold preparation}

Scaffolds were fabricated via additive manufacturing (Bioscaffolder, SysENG, Germany). Scaffolds made of poly( $\varepsilon$-caprolactone) (PCL) were produced. Briefly, the polymer was placed in a stainless syringe and processed at $100{ }^{\circ} \mathrm{C}$. The molten polymer was extruded through a cartridge unit via the application of a pressurized nitrogen flow (5 bar) and an extrusion screw rotating at $200 \mathrm{rpm}$. During plotting, fiber spacing, needle diameter, layer thickness and speed were kept constant at $650 \mu \mathrm{m}, 200 \mu \mathrm{m}, 150 \mu \mathrm{m}$ and $180 \mu \mathrm{mm} / \mathrm{min}$, respectively. The scaffolds were plotted in blocks of $20 \times 20 \mathrm{~mm}$ and $4 \mathrm{~mm}$ in height. The tested samples were $4 \times 4 \mathrm{~mm}$ cylinders punched out from the blocks. 


\section{Activation of the polymer scaffolds}

3D scaffolds were activated through immersion in a $5 \mathrm{mM}$ isopropanol solution of ethylenediamine (EDA). The reaction was allowed to proceed for 10 minutes at room temperature. Scaffolds were then rinsed with ice-cold water and finally dried in a stream of nitrogen. The aminated PCL scaffolds were immersed into $20 \mathrm{ml}$ of dry hexane and $20 \mu \mathrm{L}$ of dry pyridine, to which $20 \mu \mathrm{L}$ of 2-bromoisobutyryl bromide (BIBB) was added dropwise. The reaction mixture was gently stirred for 1 hour at room temperature to produce the 2-bromoisobutyrate- PCL surface (PCL-Br). PCL$\mathrm{Br}$ scaffolds were later-on washed repeatedly with an ethanol/water (1/1, v/v) mixture and finally dried under a stream of nitrogen.

\section{Atom transfer radical polymerization of OEGMA}

Purified OEGMA monomer ( $5 \mathrm{~g}, 9.5 \mathrm{mmol})$ and 2,2'bipyridine $(81.7 \mathrm{mg}, 0.52 \mathrm{mmol})$ were added to a water $(5 \mathrm{ml})$ and methanol $(1,26 \mathrm{ml})$ mixture. The solution was purged with argon for 30 minutes. $\mathrm{CuCl}(18.75 \mathrm{mg}, 0.19 \mathrm{mmol})$ and $\mathrm{CuBr} 2(2 \mathrm{mg}$, $0.009 \mathrm{mmol}$ ) were added into another reaction flask and also flushed with argon. Monomer, ligand and catalyst were then combined and stirred for other 30 minutes to facilitate the formation of the organometallic complex. This solution was then transferred into the flasks containing PCL-Br substrates. The flasks were sealed with rubber septa and kept at room temperature under nitrogen. Following 10 minutes of reaction time the substrates were removed from the polymerization solution, exhaustively rinsed with water to remove any unreacted compound and finally dried under a stream of nitrogen.

\section{Functionalization of PCL-POEGMA scaffolds}

POEGMA brushes on PCL scaffolds were activated by placing them in a dry DMSO solution containing $200 \mathrm{mM}$ of DSC and DMAP. Later on, the samples were incubated in a protein solution containing either $0.25 \mu \mathrm{g} / \mathrm{ml}$ BMP2 or TGF- $\beta 3$. Single protein gradients along the axial direction of the scaffolds were fabricated by placing the DSC-activated PCL-POEGMA scaffolds on top of a micro porous paper sheet previously soaked with either one of the protein solutions. The contact 
between the scaffold's outer surface and the paper reservoir was assured by placing a weight of $7.5 \mathrm{~g}$ on top of the scaffolds. Following 60 minutes of reaction time, the scaffolds were extensively rinsed with milli-Q water, and finally blow-dried with a stream of nitrogen. For the double axial gradients, first a single BMP-2 gradient was fabricated according to the procedure previously mentioned. Subsequently, the scaffold was inverted and placed on top of another micro porous paper sheet previously soaked with a TGF- $\beta 3$ solution.

\section{Cell expansion and culture}

hMSCs (male, age 22) were retrieved from the Institute of Regenerative Medicine (Temple, Texas). Briefly, a bone marrow aspirate was drawn and mononuclear cells were separated using density centrifugation. The cells were plated to obtain adherent hMSCs, which were harvested when cells reached $60-80 \%$ confluence. These were considered passage 0 (P0) cells. These P0 cells were expanded, harvested and frozen at passage 1 (P1) for distribution. Cells were grown in MSC proliferation medium, which contains minimal essential medium ( $\alpha-M E M$, Life Technologies, Bleiswijk, the Netherlands) supplemented with $10 \%$ fetal bovine serum (FBS, Lonza), $100 \mathrm{U} / \mathrm{ml}$ penicillin (Life Technologies, Bleiswijk, the Netherlands), $10 \mu \mathrm{g} / \mathrm{ml}$ streptomycin (Life Technologies, Bleiswijk, the Netherlands), $2 \mathrm{mM} \mathrm{L-glutamin} \mathrm{(Life} \mathrm{Technologies,} \mathrm{Bleiswijk,} \mathrm{the} \mathrm{Netherlands),} 0.2$ $\mathrm{mM}$ L-ascorbic acid 2-phosphate magnesium salt (ASAp, Sigma-Aldrich, Zwijndrecht, The Netherlands) and $1 \mathrm{ng} / \mathrm{ml}$ of basic fibroblast growth factor-2 (bFGF-2, Fisher Scientific, Landsmeer, the Netherlands) at $37{ }^{\circ} \mathrm{C}$ in a humid atmosphere with $5 \% \mathrm{CO}_{2}$. Cells were expanded up to approximately $80 \%$ confluency and either frozen for further use or seeded on the scaffolds.

\section{Cell seeding and culture on scaffolds}

After trypsinization with $0.25 \%$ trypsin (Life Technologies, Bleiswijk, the Netherlands), cells (passage 2-4) were counted using a Bückner chamber and resuspended in proliferation medium at a density of 500'000 cells in $40 \mu \mathrm{L}$. The day before seeding, functionalized scaffolds were disinfected in $70 \% \mathrm{EtOH}$ for $30 \mathrm{~min}$ 
under stirring, washed 3 times in phosphate buffered saline solution (PBS) (Lonza, Breda, the Netherlands), and incubated overnight in proliferation medium to allow protein adsorption on the scaffold's fibers. After protein adsorption, the $40 \mu \mathrm{L}$ of cell suspension were placed on the scaffold in a drop wise fashion to account for a cell seeding density of 500'000 cells/scaffold. The seeded scaffolds were placed for 4 hours in the incubator to allow cell adhesion before adding the cell culture medium. All the scaffolds in this study were cultured for 10 days in basic medium, consisting of the proliferation medium without the basic fibroblast growth factor-2. Scaffolds cultured with the growth factors in soluble form were cultured in basic medium supplemented with $10 \mathrm{ng} / \mathrm{mL}$ BMP-2 or TGF- $\beta 3$ (R\&D System, Abingdon, United Kingdom).

\section{DNA analysis}

The cell number per scaffold was calculated from the $\mu \mathrm{g}$ of DNA, obtained by a Cyquant DNA assay kit (Life Technologies, Bleiswijk, the Netherlands). Briefly, each scaffold was cut to improve lysis efficiency and freeze-thawed 5 times. After the freeze-thawing process, cells within the scaffolds were lysated by diluting the 20x lysis buffer provided with the kit using a saline buffer $(180 \mathrm{mM} \mathrm{NaCl}, 1 \mathrm{mM}$ EDTA in distilled water). After $1 \mathrm{~h}$ of lysis, samples were sonicated 2 times for 10 seconds using a Branson sonifier 250 (Emerson Industrial Automation, USA). DNA content was quantified with a CyQuant kit (Invitrogen, Breda, the Netherlands) according to manufacturer's protocol and fluorescence was measured at $480 \mathrm{~nm}$ using a spectrophotometer LS50B (Perkin Elmer, The Netherlands). DNA concentrations were calculated from a $\lambda$ DNA standard curve.

\section{ALP activity}

To evaluate hMSC differentiation toward the osteogenic lineage, ALP content was measured using a CDP star kit (Roche, Woerden, The Netherlands). For this purpose, $10 \mu \mathrm{L}$ of sample was added to a well of a white 96 -well plate and $40 \mu \mathrm{L}$ of substrate CDP-Star $\AA^{\circ}$, Disodium 2-chloro-5-(4-methoxyspiro \{1,2-dioxetane-3,2'-(5'chloro)tricyclo[3.3.1.13,7]decan\}-4-yl)-1-phenyl phosphate was added. After 15 
minutes incubation, luminescence was read using a spectrophotometer LS50B (Perkin Elmer) at 461-466 nm. ALP activity was corrected for DNA content.

\section{GAG amount}

To evaluate the differentiation toward the chondrogenic lineage, the GAG amount was quantified using 1,9-Dimethyl Methylene Blue (DMMB) assay. Therefore, 25 $\mu \mathrm{L}$ of sample were placed into a transparent flat bottom 96 well plate and $5 \mu \mathrm{L}$ of 2.3M NaCl solution were added, then $150 \mu \mathrm{L}$ of DMMB solution were added and absorbance was read using a Multiscan Go (Fisher Scientific, Landsmeer, the Netherlands) plate reader at a wavelength of $525 \mathrm{~nm}$. GAG content was quantified with a chondroitin standard curve and corrected for DNA content.

\section{Gene expression analysis}

For gene expression analysis the scaffolds were taken from the media washed twice with PBS, cut into small pieces and placed in an Eppendorf containing 750 $\mu \mathrm{L}$ of $\mathrm{TRIzo} \circledast \AA^{\circledR}$ (Invitrogen) and stored at $-80^{\circ} \mathrm{C}$. In the case of partition analysis the gradient scaffolds were cut in order to separate the gradient zones and the 3 samples were located in the same vial prior the addition of the TRIzol $\Theta$, in order to ensure the collection of enough RNA. RNA isolation was performed by using a Bioke RNA II nucleospin RNA isolation kit (Bioke, Leiden, The Netherlands). 150 $\mu \mathrm{L}$ of $\mathrm{CHCl}_{3}$ were added and the vials were vigorously mixed, followed by a centrifugation at $12000 \mathrm{~g}$ for 15 minutes at $4{ }^{\circ} \mathrm{C}$. The aqueous phase was transferred into a new tube and an equal amount of $70 \%$ ethanol was added. The mixture was transferred into a filter columns from the kit and the extraction was carried on by following the manufacturer's protocol. RNA concentration and purity was evaluated via an ND1000 spectrophotometer (Nanodrop Technologies, USA); CDNA was synthetized using iScript ${ }^{\mathrm{TM}}$ (BIO-RAD, Veenendaal, The Netherlands) according to manufacturer's protocol. Quantitative polymerase chain reaction (qPCR) was performed on the obtained cDNA by using the $i Q S Y B R B G$ ree Supermix (BIO-RAD, Veenendaal, The Netherlands) and the primers listed in Table 1. PCR reaction was carried out on the MyiQ2 Two-Color Real-Time PCR 
Detection System (BIO-RAD, Veenendaal, The Netherlands) under the following conditions, the cDNA was denatured for 10 minutes at $95{ }^{\circ} \mathrm{C}$, followed by 45 cycles, consisting of 15 seconds at $95^{\circ} \mathrm{C}, 15$ seconds at $60^{\circ} \mathrm{C}$ and 15 seconds at $72^{\circ} \mathrm{C}$. A melting curve was generated from each reaction to test the presence of primer dimers and aspecific product. The cycle threshold was calculated by the Bio-Rad iQ5 optical system software, in which the threshold was set in the lower log-linear region of the fluorescent signal. Ct values were normalized by the B2M housekeeping gene and $\Delta \mathrm{Ct}$ ((average of $\mathrm{Ct}$ control)-Ct value). Results were expressed as fold induction in mRNA expression normalized to the gene expression of the PCL control scaffolds cultured in basic medium.

\begin{tabular}{|c|c|c|}
\hline Gene & Forward Primer & Reverse Primer \\
\hline B2M & ACAAAGTCACATGGTTCACA & GACTTGTCTTTCAGCAAGGA \\
\hline Aggrecan & AGGCAGCGTGATCCTTACC & GGCCTCTCCAGTCTCATTCTC \\
\hline Sox9 & TGGGCAAGCTCTGGAGACTTC & ATCCGGGTGGTCCTTCTTGTG \\
\hline Collagen Ila & CGTCCAGATGACCTTCCTACG & TGAGCAGGGCCTTCTTGAG \\
\hline Runx2 & TGGTTACTGTCATGGCGGGTA & TCTCAGATCGTTGAACCTTGCTA \\
\hline ALP & ACAAGCACTCCCACTTCATC & TTCAGCTCGTACTGCATGTC \\
\hline $\begin{array}{c}\text { Bone } \\
\text { sialoprotein }\end{array}$ & CCCCACCTTTTGGGAAAACCA & TCCCCGTTCTCACTTTCATAGAT \\
\hline Osteocalcin & TGAGAGCCCTCACACTCCTC & CGCCTGGGTCTCTTCACTAC \\
\hline
\end{tabular}

Table 3. Table showing the genes analyzed and the forward and reverse primers used for the PCR.

\section{Fluorescent staining}

After functionalization the PCL scaffolds were cut in half and incubated with PBSBSA for 30 minutes. Same procedure was performed on non-functionalized PCL scaffolds. A goat polyclonal antibody against human BMP-2 was resuspended according to the supplier in a PBS-BSA solution up to a concentration of 200 $\mu \mathrm{g} / \mathrm{mL}$. The scaffolds were covered with a PBS-BSA solution containing the antibody with a dilution of 1:200 and incubated 1 hour at room temperature. Scaffolds were incubated for another hour at room temperature in a PBS-BSA solution containing a polyclonal rabbit anti-goat coupled with the fluorophore Alexa 568, at a dilution of 1:1000. POEGMA-funcitonalized scaffolds and PCL scaffolds 
were incubated in a solution containing the growth factor. On the supernatant a BMP-2 Elisa (Sigma-Aldrich, Zwijndrecht, The Netherlands) was performed. The measured amount was subtracted from the original concentration and expressed as percentage.

\section{Statistical analysis}

All the quantitative data are expressed as mean \pm standard deviation. Statistics were performed using IBM SPSS Statistics 20. A two-way ANOVA with Tukey as post-hoc test were used. Differences between experimental groups were considered significant when $p<0.05$ and indicated with $\left(^{*}\right)$.

\section{Acknowledgements}

This project was funded by the Dutch Technology Foundation STW (Grant number 11135). This project/research has been also made possible with the support of the Dutch Province of Limburg. Some of the materials used in this work were provided by the Texas A\&M Health Science Center College of Medicine Institute for Regenerative Medicine at Scott \& White through a grant from NCRR of the NIH (Grant \#P40RR017447).

\section{References}

1. Leatrese D. Harris, B.-S.K., David J. Mooney, Open pore biodegradable matrices formed with gas foaming. 1998.

2. Suh, S.W., et al., Effect of Different Particles on Cell Proliferation in Polymer Scaffolds Using a Solvent-Casting and Particulate Leaching Technique. ASAIO Journal, 2002. 48(5): p. 460464.

3. Levingstone, T.J., et al., A biomimetic multi-layered collagen-based scaffold for osteochondral repair. Acta Biomaterialia, 2014. 10(5): p. 1996-2004.

4. Sin, D., et al., Polyurethane (PU) scaffolds prepared by solvent casting/particulate leaching (SCPL) combined with centrifugation. Materials Science and Engineering: $C, 2010.30(1): p$. 78-85.

5. Gunnewiek, M.K., et al., Creeping proteins in microporous structures: polymer brush-assisted fabrication of 3D gradients for tissue engineering. Adv Healthc Mater, 2015. 4(8): p. 1169-74.

6. Park, J.S., et al., The effect of matrix stiffness on the differentiation of mesenchymal stem cells in response to TGF-beta. Biomaterials, 2011. 32(16): p. 3921-30.

7. Johnstone, B., et al., In vitro chondrogenesis of bone marrow-derived mesenchymal progenitor cells. Exp Cell Res, 1998. 238(1): p. 265-72. 
8. Re'em, T., et al., Simultaneous regeneration of articular cartilage and subchondral bone induced by spatially presented TGF-beta and BMP-4 in a bilayer affinity binding system. Acta Biomater, 2012. 8(9): p. 3283-93.

9. Chen, G., C. Deng, and Y.-P. Li, TGF- $\beta$ and BMP Signaling in Osteoblast Differentiation and Bone Formation. International Journal of Biological Sciences, 2012. 8(2): p. 272-288.

10. Mohan, N., et al., Continuous gradients of material composition and growth factors for effective regeneration of the osteochondral interface. Tissue Eng Part A, 2011. 17(21-22): p. 2845-55.

11. Reyes, R., et al., Repair of an osteochondral defect by sustained delivery of BMP-2 or TGFbeta1 from a bilayered alginate-PLGA scaffold. J Tissue Eng Regen Med, 2014. 8(7): p. 521-33.

12. Wang, X., et al., Growth factor gradients via microsphere delivery in biopolymer scaffolds for osteochondral tissue engineering. J Control Release, 2009. 134(2): p. 81-90.

13. Suarez-Gonzalez, D., et al., Controlled multiple growth factor delivery from bone tissue engineering scaffolds via designed affinity. Tissue Eng Part A, 2014. 20(15-16): p. 2077-87.

14. Whitaker, M.J., et al., Growth factor release from tissue engineering scaffolds. J Pharm Pharmacol, 2001. 53(11): p. 1427-37.

15. Zara, J.N., et al., High doses of bone morphogenetic protein 2 induce structurally abnormal bone and inflammation in vivo. Tissue Eng Part A, 2011. 17(9-10): p. 1389-99.

16. Tannoury, C.A. and H.S. An, Complications with the use of bone morphogenetic protein 2 (BMP-2) in spine surgery. Spine J, 2014. 14(3): p. 552-9.

17. James, A.W., et al., A Review of the Clinical Side Effects of Bone Morphogenetic Protein-2. Tissue Eng Part B Rev, 2016. 22(4): p. 284-97.

18. Di Luca, A., C. Van Blitterswijk, and L. Moroni, The osteochondral interface as a gradient tissue: From development to the fabrication of gradient scaffolds for regenerative medicine. Birth Defects Research Part C: Embryo Today: Reviews, 2015. 105(1): p. 34-52.

19. Daopin, S., et al., Crystal structure of transforming growth factor-beta 2: an unusual fold for the superfamily. Science, 1992. 257(5068): p. 369-73.

20. Migliorini, E., et al., Tuning cellular responses to BMP-2 with material surfaces. Cytokine \& growth factor reviews, 2016. 27: p. 43-54.

21. Luo, Z., et al., Mechano growth factor (MGF) and transforming growth factor (TGF)-beta3 functionalized silk scaffolds enhance articular hyaline cartilage regeneration in rabbit model. Biomaterials, 2015. 52: p. 463-75.

22. Lecanda, F., L.V. Avioli, and S.L. Cheng, Regulation of bone matrix protein expression and induction of differentiation of human osteoblasts and human bone marrow stromal cells by bone morphogenetic protein-2. J Cell Biochem, 1997. 67(3): p. 386-96.

23. Gori, F., et al., Differentiation of human marrow stromal precursor cells: bone morphogenetic protein-2 increases OSF2/CBFA1, enhances osteoblast commitment, and inhibits late adipocyte maturation. J Bone Miner Res, 1999. 14(9): p. 1522-35.

24. Diefenderfer, D.L., et al., BMP responsiveness in human mesenchymal stem cells. Connect Tissue Res, 2003. 44 Suppl 1: p. 305-11.

25. Lysdahl, H., et al., Preconditioning Human Mesenchymal Stem Cells with a Low Concentration of BMP2 Stimulates Proliferation and Osteogenic Differentiation In Vitro. Biores Open Access, 2014. 3(6): p. 278-85.

26. Komori, T., et al., Targeted disruption of Cbfa1 results in a complete lack of bone formation owing to maturational arrest of osteoblasts. Cell, 1997. 89(5): p. 755-64.

27. Komori, T., Regulation of osteoblast differentiation by transcription factors. J Cell Biochem, 2006. 99(5): p. 1233-9.

28. Wang, L., et al., Dynamic Expression Profiles of Marker Genes in Osteogenic Differentiation of Human Bone Marrow-derived Mesenchymal Stem Cells. Chin Med Sci J, 2015. 30(2): p. 108-13.

29. Worster, A.A., et al., Effect of transforming growth factor beta1 on chondrogenic differentiation of cultured equine mesenchymal stem cells. Am J Vet Res, 2000. 61(9): p. 1003-10.

30. Vater, C., P. Kasten, and M. Stiehler, Culture media for the differentiation of mesenchymal stromal cells. Acta Biomater, 2011. 7(2): p. 463-77.

31. Song, L. and R.S. Tuan, Transdifferentiation potential of human mesenchymal stem cells derived from bone marrow. FASEB J, 2004. 18(9): p. 980-2. 
32. Lee, H.J., et al., Changes in surface markers of human mesenchymal stem cells during the chondrogenic differentiation and dedifferentiation processes in vitro. Arthritis Rheum, 2009. 60(8): p. 2325-32.

33. Miller, E.D., et al., Inkjet printing of growth factor concentration gradients and combinatorial arrays immobilized on biologically-relevant substrates. Comb Chem High Throughput Screen, 2009. 12(6): p. 604-18.

34. Cabanas-Danés, J., et al., A Supramolecular Host-Guest Carrier System for Growth Factors Employing VHH Fragments. Journal of the American Chemical Society, 2014. 136(36): $\mathrm{p}$. 12675-12681.

35. Bilgen, B., et al., FBS suppresses TGF-beta1-induced chondrogenesis in synoviocyte pellet cultures while dexamethasone and dynamic stimuli are beneficial. J Tissue Eng Regen Med, 2007. 1(6): p. 436-42.

36. Chen, F.M., M. Zhang, and Z.F. Wu, Toward delivery of multiple growth factors in tissue engineering. Biomaterials, 2010. 31(24): p. 6279-308.

37. Simmons, C.A., et al., Dual growth factor delivery and controlled scaffold degradation enhance in vivo bone formation by transplanted bone marrow stromal cells. Bone, 2004. 35(2): p. 562-9.

38. Raiche, A.T. and D.A. Puleo, In vitro effects of combined and sequential delivery of two bone growth factors. Biomaterials, 2004. 25(4): p. 677-85.

39. Holland, T.A., Y. Tabata, and A.G. Mikos, Dual growth factor delivery from degradable oligo(poly(ethylene glycol) fumarate) hydrogel scaffolds for cartilage tissue engineering. J Control Release, 2005. 101(1-3): p. 111-25.

40. Balasubramanian, V., et al., Protein delivery: from conventional drug delivery carriers to polymeric nanoreactors. Expert Opin Drug Deliv, 2010. 7(1): p. 63-78.

41. Jaklenec, A., et al., Sequential release of bioactive IGF-I and TGF- $\beta 1$ from PLGA microsphere-based scaffolds. Biomaterials, 2008. 29(10): p. 1518-1525. 


\section{Supporting information}

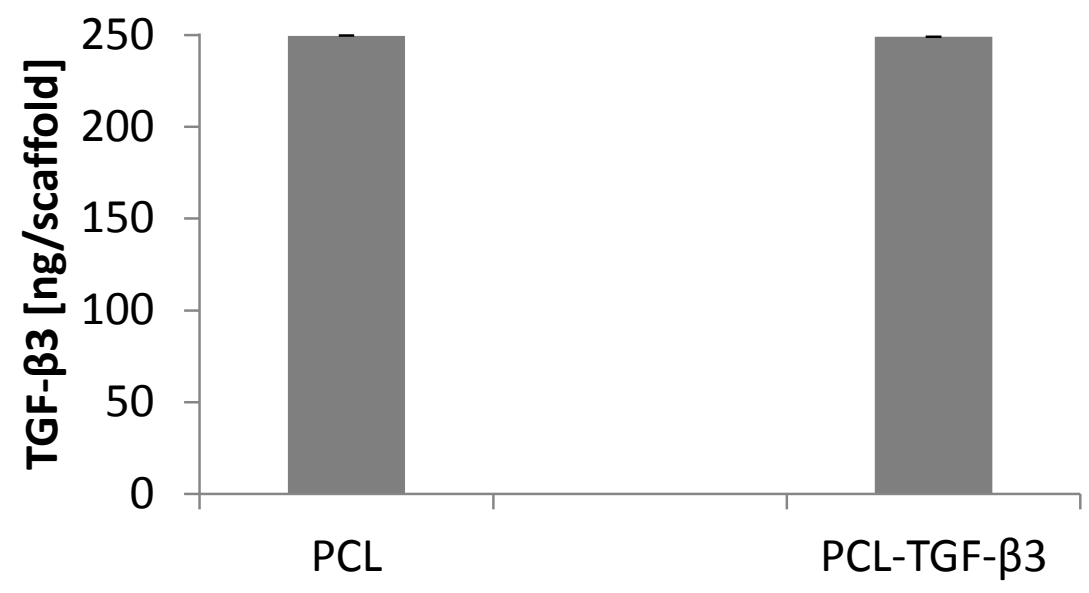

Figure S1. Plot showing the TGF- $\beta 3$ amount adsorbed on PCL scaffolds and linked to the PCL-POEGMA brushes scaffolds before the washing steps after the functionalization.

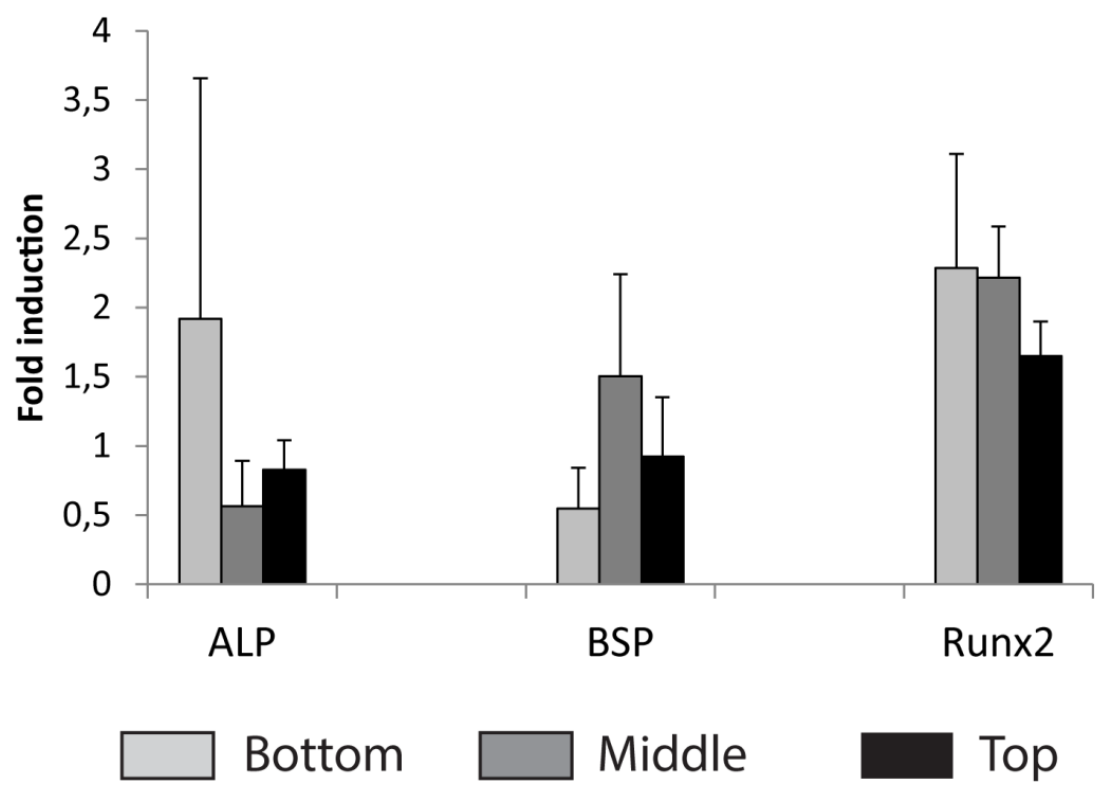

Figure S2. Plot showing the ALP, BSP and runx2 for PCL scaffolds functionalized with BMP-2 in a gradient fashion in the different scaffold areas. 


\section{General discussion}

Human mesenchymal stem cells are known to differentiate toward several cell lines among which osteoblasts and chondrocytes [1-4]. Due to this ability they are mistakenly referred as "stem cells" although they lack part of the characteristics which are shared among stem cells. Embryonic stem cells and tumor cells are associated with immortality, due to the presence of an enzyme, named telomerase, which prevents the shortening of their telomeres. Small amount of these enzyme was found in adult stem cells of the gut, skin and hematopoietic system [5]. Lacking this enzyme [6], human mesenchymal stromal cells have a different telomere biology than adult stem cells. As hMSCs have only a finite ability for self-renewal like most somatic cells [7], the definition of human mesenchymal stromal cells better applies to this cell population.

The effort of regenerative medicine is to empower this differentiation in vitro after expansion on 3D structures (scaffolds) before their re-implantation in vivo to heal a defect [8-10]. After giving a short synopsis of this thesis, in chapter 1 the osteochondral tissue is presented as a gradient tissue allowing the smooth transition from the mineralized subchondral bone to the soft and hydrated cartilage. Additionally, an overview of the most recent advances in the use of bi- and trilayered scaffolds in animal models and in clinical trials is presented. Although the use of gradient structures has received increased attention in the last years, an analysis of the most common cues leading to osteogenic and chondrogenic lineage in the same construct has not been fully presented. In chapters 2 and 3, scaffolds with an in built gradient in pore size were analyzed. The pore size increased along the $z$ axis and 4 zones were fabricated with pore size of $326 \mu \mathrm{m} \pm 17 \mu \mathrm{m}, 540 \mu \mathrm{m} \pm$ $11 \mu \mathrm{m}, 744 \mu \mathrm{m} \pm 16 \mu \mathrm{m}$ and $968 \mu \mathrm{m} \pm 25 \mu \mathrm{m}$.. The presence of a gradient structure didn't vary the cell attachment on the scaffolds, but significantly improved the differentiation of hMSCs compared to scaffolds with homogenous pore size in the presence of differentiation soluble factors in the culture medium. By analyzing the differentiation toward the osteogenic and the chondrogenic lineage of hMSCs along the $z$ axis, an opposite trend was observed. Expression of chondrogenic biochemical and genetic markers increased with decreasing of the pore size, while 
osteogenic markers followed the opposite trend, increasing with the increasing in pore size. This different behavior can be explained by the design of the scaffold. The differences in pore size could have generated a hypoxic microenviroment with lower nutrient availability in the portion with small pores, which increases in portions with larger pore size. It was proven that hypoxia is hampering osteogenic hMSC differentiation but contributing to chondrogenic differentiation in vitro [11, 12]. Smaller pores closed faster and the resulting high cell density might have caused an environment with lower oxygen and nutrients availability eventually leading to the observed trends. The increased chondrogenic differentiation from the large to the smaller pore size could be due to an increased cell density. As in vivo chondrogenesis starts with cell condensation triggered by TGF- $\beta$ [13], the small pores were filled faster leading the cells to an aggregate-like state that might have enhanced the chondrogenic cascade and the expression of chondrogenic markers. Oh et al. [14] determined the ideal pore size for chondrocyte growth in PCL scaffolds to be $370-400 \mu \mathrm{m}$. In our scaffolds, the highest differentiation toward this lineage was achieved in the portion with $326 \pm 17 \mu \mathrm{m}$, very close to the one showed by the fore mentioned paper. Further studies are needed to elucidate how the gradient in pore size can be applied in osteochondral regeneration. A first step might be a skin pocket study in mice to define the tissue ingrowth in each scaffold compartment, and understand if the current pore size is enough to ensure tissue ingrowth in each compartment. If successful tissue ingrowth I achieved, an orthotopic animal model could be proposed as a follow-up study. The proof of concept presented in this thesis showed an increased differentiation toward the osteogenic and chondrogenic lineage under the effect of soluble factors. The solely pore size is not enough to start and support hMSC differentiation. Using the gradient scaffolds in a rabbit microfracture model would ensure the infiltration of hMSCs from the bone marrow and the presence of the osteogenic signals from the subchondral bone and the chondrogenic signals from the cartilage surrounding areas. Ideally, the porosity gradient scaffolds would be inserted with the biggest pore size in contact with the subchondral bone and the smallest pore size close to the joint surface. 
In chapter 4 another gradient in pore geometry was analyzed. By varying the fiber deposition pattern, a gradient in pore shape was achieved along the $z$ axis. A 90ㅇ angle between the fibers of consecutive layers led to squared pores. By changing the deposition angle to $45^{\circ}, 30^{\circ}$ and $15^{\circ}$ pores assumed a rhomboidal shape with a continuous increase in the major diagonal and a decrease of the minor diagonal. The variation in pore shape determined a variation in pore size as well. The gradient in pore shape didn't improve cell seeding efficiency with respect to scaffolds with homogeneous pore geometry. However, the amount of ECM deposited was significantly enhanced in gradient structures compared to homogeneous ones. By SEM analysis, it was possible to see how the hMSCs tended to wrap the fibers in the control scaffolds with some rare fiber bridging. Conversely, the cross section of the gradient scaffolds revealed a sparse ECM present in all the compartments of the scaffolds. This may be due to the increased fiber connection. Bidan et al. proved that the pore closure begins at the edge of the pores, by culturing pre-osteoblasts [15]. The increased number of fiber connections stimulated the cells in depositing ECM by closing the pore from the point where the fibers met each other toward the center of the pore. This resulted in a significantly higher ECM deposition in the gradient scaffolds with respect to scaffolds presenting an homogenous geometry. Similarly to what observed in porosity gradients, an opposite trend along the $z$ axis of the pore geometry gradient was observed. Chondrogenic biochemical and genetic markers were enhanced in squared pores and decreased along the $z$ axis toward the rhomboidal pores. Osteogenic markers, on the other hand, showed their maximum expression in the rhomboidal pores and reached the minimum in the squared pores. This was proven separately under osteogenic and chondrogenic conditions as well as in medium containing both osteogenic and chondrogenic stimuli. Domingos et al. defined a strong correlation between hMSC viability and scaffold pore size and geometry [16]. On the other hand, they didn't find any correlation between pore size and geometry, and hMSC morphology. The correlation between pore geometry and hMSC differentiation described in chapter 4 was present only in supplemented media. hMSCs cultured under basic conditions didn't show any sign of differentiation and no variation in osteogenic or chondrogenic markers among the gradient zones. Acting on the 
solely geometry of the pores can enhance the differentiation of the cells growing within them, but it is not enough to start the process. In the perspective of using these gradient scaffolds sin a microfracture operation, the simple application of the scaffold within the site should promote the infiltration of the bone marrow containing hMSCs. As earlier discussed, it is reasonable to expect that osteogenic signals would come from the subchondral bone and the chondrogenic ones from the cartilage surrounding areas.

Among the cues determining hMSC fate, the substrate physicochemical characteristics play an important role [17-19]. In chapter 6, the differentiation of hMSCs in scaffolds presenting a gradient in surface energy and in surface and bulk stiffness was analyzed. Both gradients were achieved by plotting 3 materials in the same scaffolds. The chosen materials were PLLA, PCL and 300PEOT55PBT45. The chosen materials are all FDA approved for specific medical applications. PLA and PCL had already found several application for bone regeneration and were applied in a number of animal studies [8, 20, 21], whereas 300PEOT55PBT45 was used in cartilage regeneration reaching clinical evaluation [22]. Before plotting, the materials were characterized by contact angle for surface energy measurement and via uniaxial compression and AFM for bulk and surface stiffness respectively. PCL showed the highest surface energy, 300PEOT55PBT45 was plotted after it, finally followed by PLA. The stiffer material was PLA, followed by PCL and 300PEOT55PBT45. Wettability gradient after 7 days under chondrogenic conditions displayed significantly higher GAG deposition with respect to stiffness gradient and single material scaffolds. After 28 days under osteogenic conditions, 300PEOT55PBT45 scaffolds displayed significantly higher levels of ALP, whereas under chondrogenic conditions all the single material based scaffolds outperformed both gradients. When gradient portions were analyzed, several differences were showed among the materials composing the stiffness and wettability gradients. Many studies have highlighted the effect of the physicochemical properties of the culturing substrate on hMSC differentiation [17-19, 23-25]. In particular, several studies highlighted the beneficial effect of stiffer substrate on hMSC osteogenic differentiation [26-28]. In contrast with literature data, 300PEOT55PBT45 showed the highest ALP levels after 7 days under osteogenic conditions, whereas higher 
ALP activity was expected on the PLA compartment with a decreasing trend toward the 300PEOT55PBT45 side. After 28 days under osteogenic conditions, ALP levels were similar in all the stiffness gradient compartments. hMSCs cultured under chondrogenic conditions didn't show any differences among gradient zones.

Wettability gradient scaffolds showed, instead, a more specific influence of the different biomaterials used for fabrication. After 28 days, 300PEOT55PBT45 showed again the highest ALP levels under osteogenic conditions compared to the other full scaffolds. The optimum contact angle for hMSC osteogenic differentiation in presence of biological stimuli ranges between 40 and 90 degrees [29]. All the chosen materials fell into this range. Under chondrogenic conditions within the wettability gradient, PLLA showed significantly higher levels of GAG compared to 300PEOT55PBT45 and PCL. According to Glennon Alty et al., there is no direct correlation between contact angle and chondrogenic differentiation [30]. Therefore, further studies are needed to elucidate the role of multimaterial scaffolds on osteochondral regeneration. A first approach could be implementing the study presented in this thesis with a longer culture time and a wider panel of osteogenic and chondrogenic markers. Despite the fact that we have selected biomateirals that showed already in literature to be supportive for skeletal regeneration $[21,22$, 31-33], it might be that the same multimaterial concept could be more efficient with new biomaterial formulations [34]. Additionally, animal studies will help to understand the differences in tissue ingrowth within the different gradient compartments in wettability and stiffness gradient.

A further indication on how the oxygen and nutrient diffusion played a pivotal role in hMSC differentiation in AM scaffolds is showed in chapter 5, where the designed constructs were significantly different than the ones displayed in the first chapters of the thesis concerning structural gradients. The scaffolds were designed as a cylinder in a tube in a tube, and were composed by an inner cylinder included in two rings one into another. When changing pore size in this scaffold configuration, a radial pore size gradient could be fabricated. In the radial gradient, not only the size of the pores but also their localization affected the differentiation of hMSCs residing in the gradient portion. ALP activity of seeded hMSCs was increased in all the external portion of the scaffolds, independently from whether the pore size was 
increased from the inside to outside or vice versa. Conversely, the expression of the osteogenic markers BSP and Runx2 was significantly higher in the areas with the smallest pore size, independently from their localization within the scaffold. Cadherins are proteins responsible for cell-cell adhesion which control cell proliferation, differentiation and survival of mesenchymal cells [35, 36]. N-cadherin expression increases in the early stages of osteoblast differentiation and this is associated with increased cell-cell interactions along with cell-cell gap junctions, which are required for full expression of the osteoblastic phenotype [37-39]. Therefore, it might be that the higher cell density led to a higher differentiation rate. The discrepancy in results with the axial porosity gradient may rely in the structure of the scaffolds. Axial porosity gradient scaffolds were cylinders of $4 \times 4 \mathrm{~mm}$ and showed portion of the same size with different pore size. Radial scaffolds were cylinders of $16 \times 3.6 \mathrm{~mm}$, with portion showing significantly different sizes. All in all, a direct comparison between the axial and radial porosity gradient is too speculative to attempt. Bone fractures have the ability to heal themselves when the distance between the 2 extremities is below $2 \mathrm{~cm}$. In case of severe and complicated fractures this self-healing ability is missing. A possible application for the radial scaffolds might be as a smart implant to be placed in between two extremities or when part of a long bone must be resected by surgery. Despite being a first step towards mimicking the radial structural properties of cortical bone, the model presented in chapter 6 is still very simplistic and additional studies are necessary to further understand the behavior of hMSCs in these scaffolds. Ideally, a CT scan of the missing bone can be acquired and used to print a bone implant with the same size and shape presenting the gradient described in chapter 6 to mimic the compactness of the bone in the radial direction.

Another way to generate scaffolds presenting a gradient can be developed by exploiting the capillary forces that are generated within the scaffold's pore network for functionalization with biological moieties. In chapter 7 , scaffolds were functionalized with nano-brushes in order to allow the attachment of bioactive molecules on their fiber surface in a gradient fashion. The scaffolds had a highly interconnected pore network with pores of 650 um, which determined the generation of capillary forces. Once the brushes-covered scaffolds entered in 
contact with the reservoir containing the targeted bioactive molecule, the solution was driven to the top of the scaffold by the capillary forces. This gradual filling of the pores put different areas of the scaffold in contact with the bioactive containing solution for different time, resulting in a higher functionalization on one side of the scaffold, decreasing toward the other extremity. In the radial gradient configuration, the same forces moved the solution from the center toward the external side of the scaffolds. This method is suitable for a future use in clinical practice due to its simplicity and rapidity. hMSC density and position within the 3D structure plays a pivotal role in their differentiation $[40,41]$. Hence, their 3D positioning could be directed by the addition of a bioadhesive molecule such as fibronectin. The nonadhesive PEOEGMA nano-brushes allowed the attachment of cells only in the direction of the gradients, either in an axial or radial configuration. The use of nanobrushes as linkers for the functionalization of the scaffolds with bioactive molecules was proven by generating an opposite axial gradient of fluorescently labelled bovine serum albumin. The choice of this protein was based as a proof of concept for the functionalization of the scaffolds in chapter 8 with bone morphogenetic protein -2 (BMP-2) and transforming growth factor - $\beta 3$ (TGF- $\beta 3$ ), in order to stimulate hMSC skeletal differentiation in a gradient fashion.

The use of non-biofouling molecules as linkers allowed cell attachment only where the bioactive molecules were present. RGD-like aminoacid sequences present in the biomolecule structure served as anchoring point. Then, the constant activation of the receptor by the ligand would have played a stimulatory effect on the hMSC differentiation toward the osteogenic and chondrogenic lineage. Following this reasoning, BMP-2 binds its receptor complex BMPR I and II, which determines the activation of the Smad cascade. Phosphorilated Smad 1/5/8 binds Smad 4. Via this binding the complex is transferred to the nucleus where it acts as transcription factor and regulates the expression of transcriptional factors and transcriptional coactivators important in osteoblasts such as distal-less homeobox 5 (DIx5), Runtrelated transcription factor 2 (Runx2) and osterix (Osx) [42-45].

TGF- $\beta 3$ has two receptors type I and type II. The binding of TGF- $\beta 3$ to its receptor complex TGF $\beta$ RII and type I in the form of Activin-like-kinase (ALK) 4, 5 or 7 , determines the phosphorylation of the Smad2/3 complex and its combination with 
Smad4. The complex Smad $2 / 3 / 4$ is then able to translocate in the nucleus and promotes the expression of chondrogenic genes such as Sox9, collagen type II and aggrecan. [45-50]. The stimulatory effect observed in chapter 8 resulted in significantly improving both osteogenic and chondrogenic differentiation of hMSCs. Cells growing on non-functionalized scaffolds but in contact with media supplemented with soluble BMP-2 or TGF- $\beta 3$ displayed significantly lower expression of osteogenic and chondrogenic markers respectively. The growth factor gradient scaffolds, however, didn't show the same effect as the fully functionalized scaffolds, probably due to a too low concentration of bioactive molecules per surface area. The immersion of the scaffolds in the growth factorcontaining solution might have been more effective than the complexation by capillary forces. Additionally, the stimulatory effect on the fully functionalized scaffolds was more effective due to a sort of self-sustaining process. We could hypothesize that cells adhering directly to the functionalized fibers were stimulated toward the osteogenic or chondrogenic lineages. Cells filling the pores, therefore not in direct contact with the growth factors, were exposed to the ECM produced by the differentiating cells, which presumably generated a differentiation stimulating environment within each pore. The lower amount of growth factors adhering on the gradient scaffolds might have resulted in a lack of this self-sustaining effect. The proof of concept presented in chapter 8 with fibronectin worked since fibronectin was acting as an anchoring point for the cell. Similarly, in chapter 9 cell adhesion in gradient scaffolds followed the gradient of BMP-2 and TGF- $\beta 3$. Cell differentiation is a more fine regulated process compared to cell adhesion, therefore more difficult to be achieved by gradient functionalization of growth factors at the tested concentration. In literature, several studies implied the use of growth factors used alone or in combination with scaffolds for their sustained release [51-54]. The main limitation when using growth factors is the use of high doses, which may cause adverse effect beside their prohibitive costs [55-57]. The studies presented so far to generate gradients of growth factors in 3D were based on complex chemical processes, which would make their application in the clinics very complicated due to the material used and the complexity of the process $[51,52,58]$. The method 
presented in chapter 9 needs further optimization, but the principle proved in chapter 8 is promising for clinical translation due to its simplicity. 


\section{References}

1. Re'em, T., et al., Simultaneous regeneration of articular cartilage and subchondral bone induced by spatially presented TGF-beta and BMP-4 in a bilayer affinity binding system. Acta Biomater, 2012. 8(9): p. 3283-93.

2. Augello, A. and C. De Bari, The regulation of differentiation in mesenchymal stem cells. Hum Gene Ther, 2010. 21(10): p. 1226-38.

3. Pittenger, M.F., et al., Multilineage Potential of Adult Human Mesenchymal Stem Cells. Science, 1999. 284(5411): p. 143-147.

4. Abdallah, B.M. and M. Kassem, Human mesenchymal stem cells: from basic biology to clinical applications. Gene Ther, 2008. 15(2): p. 109-16.

5. Zimmermann, S., et al., Lack of telomerase activity in human mesenchymal stem cells. Leukemia, 2003. 17(6): p. 1146-9.

6. Tichon, A., et al., Telomerase activity and expression in adult human mesenchymal stem cells derived from amyotrophic lateral sclerosis individuals. Cytotherapy, 2009. 11(7): p. 837-48.

7. Samsonraj, R.M., et al., Telomere length analysis of human mesenchymal stem cells by quantitative PCR. Gene, 2013. 519(2): p. 348-55.

8. Anderson, J.A., et al., Stem Cell Therapies for Knee Cartilage Repair: The Current Status of Preclinical and Clinical Studies. The American Journal of Sports Medicine, 2014. 42(9): p. 2253-2261.

9. Rodrigues, M.T., M.E. Gomes, and R.L. Reis, Current strategies for osteochondral regeneration: from stem cells to pre-clinical approaches. Current Opinion in Biotechnology, 2011. 22(5): p. 726-733.

10. Hunziker, E.B., Articular cartilage repair: basic science and clinical progress. A review of the current status and prospects. Osteoarthritis and Cartilage, 2002. 10(6): p. 432-463.

11. Hsu, S.H., C.T. Chen, and Y.H. Wei, Inhibitory effects of hypoxia on metabolic switch and osteogenic differentiation of human mesenchymal stem cells. Stem Cells, 2013. 31(12): p. 2779-88.

12. Preeti Malladi, Y.X., Michael Chiou, Amato J. Giaccia, and Michael T. Longaker, Effect of reduced oxygen tension on chondrogenesis and osteogenesis in adipose-derived mesenchymal cells. Am J Physiol Cell Physiol, 2006. 290: p. 1139-1146.

13. Di Luca, A., C. Van Blitterswijk, and L. Moroni, The osteochondral interface as a gradient tissue: From development to the fabrication of gradient scaffolds for regenerative medicine. Birth Defects Research Part C: Embryo Today: Reviews, 2015. 105(1): p. 34-52.

14. Oh, S.H., et al., In vitro and in vivo characteristics of $P C L$ scaffolds with pore size gradient fabricated by a centrifugation method. Biomaterials, 2007. 28(9): p. 1664-71.

15. Bidan, C.M., et al., Geometry as a factor for tissue growth: towards shape optimization of tissue engineering scaffolds. Adv Healthc Mater, 2013. 2(1): p. 186-94.

16. Domingos, M., et al., The first systematic analysis of 3D rapid prototyped poly( $\varepsilon$-caprolactone) scaffolds manufactured through BioCell printing: the effect of pore size and geometry on compressive mechanical behaviour and in vitro hMSC viability. Biofabrication, 2013. 5(4): p. 045004.

17. Tse, J.R. and A.J. Engler, Stiffness Gradients Mimicking In Vivo Tissue Variation Regulate Mesenchymal Stem Cell Fate. PLoS ONE, 2011. 6(1): p. e15978.

18. Yeung, T., et al., Effects of substrate stiffness on cell morphology, cytoskeletal structure, and adhesion. Cell Motil Cytoskeleton, 2005. 60(1): p. 24-34.

19. Park, J.S., et al., The effect of matrix stiffness on the differentiation of mesenchymal stem cells in response to TGF-beta. Biomaterials, 2011. 32(16): p. 3921-30.

20. Aydin, H.M., A Three-Layered Osteochondral Plug: Structural, Mechanical, and in vitro Biocompatibility Analysis. Advanced Engineering Materials, 2011. 13(12): p. B511-B517.

21. Ding, C., et al., Regeneration of a goat femoral head using a tissue-specific, biphasic scaffold fabricated with CAD/CAM technology. Biomaterials, 2013. 34(28): p. 6706-16.

22. Bartha, L., et al., A clinical feasibility study to evaluate the safety and efficacy of PEOT/PBT implants for human donor site filling during mosaicplasty. Eur J Orthop Surg Traumatol, 2013. 23(1): p. 81-91.

23. Wen, J.H., et al., Interplay of matrix stiffness and protein tethering in stem cell differentiation. Nat Mater, 2014. 13(10): p. 979-987. 
24. Peyton, S.R., et al., Marrow-derived stem cell motility in 3D synthetic scaffold is governed by geometry along with adhesivity and stiffness. Biotechnol Bioeng, 2011. 108(5): p. 1181-93.

25. Even-Ram, S., V. Artym, and K.M. Yamada, Matrix control of stem cell fate. Cell, 2006. 126(4): p. 645-7.

26. Dupont, S., et al., Role of YAP/TAZ in mechanotransduction. Nature, 2011. 474(7350): p. 179-83.

27. Sikavitsas, V.I., J.S. Temenoff, and A.G. Mikos, Biomaterials and bone mechanotransduction. Biomaterials, 2001. 22(19): p. 2581-93.

28. Chatterjee, K., et al., The effect of 3D hydrogel scaffold modulus on osteoblast differentiation and mineralization revealed by combinatorial screening. Biomaterials, 2010. 31(19): p. 505162.

29. Hao, L., et al., Directing the fate of human and mouse mesenchymal stem cells by hydroxylmethyl mixed self-assembled monolayers with varying wettability. J Mater Chem B Mater Biol Med, 2014. 2(30): p. 4794-4801.

30. Glennon-Alty, L., et al., Induction of mesenchymal stem cell chondrogenesis by polyacrylate substrates. Acta Biomater, 2013. 9(4): p. 6041-51.

31. Frenkel, S.R., et al., Regeneration of articular cartilage - Evaluation of osteochondral defect repair in the rabbit using multiphasic implants. Osteoarthritis and Cartilage, 2005. 13(9): p. 798-807.

32. <Use-of-Osteoplug-polycaprolactone.pdf>.

33. Higuera, G.A., et al., In vivo screening of extracellular matrix components produced under multiple experimental conditions implanted in one animal. Integr Biol (Camb), 2013. 5(6): p. 889-98.

34. Chen, H., et al., Tailoring chemical and physical properties of fibrous scaffolds from block copolyesters containing ether and thio-ether linkages for skeletal differentiation of human mesenchymal stromal cells. Biomaterials, 2016. 76: p. 261-72.

35. Wheelock, M.J. and K.R. Johnson, Cadherin-mediated cellular signaling. Curr Opin Cell Biol, 2003. 15(5): p. 509-14.

36. Gumbiner, B.M., Regulation of cadherin-mediated adhesion in morphogenesis. Nat Rev Mol Cell Biol, 2005. 6(8): p. 622-34.

37. Schiller, P.C., et al., Gap-junctional communication is required for the maturation process of osteoblastic cells in culture. Bone, 2001. 28(4): p. 362-9.

38. Ferrari, S.L., et al., A role for N-cadherin in the development of the differentiated osteoblastic phenotype. J Bone Miner Res, 2000. 15(2): p. 198-208.

39. Cheng, S.L., et al., Human osteoblasts express a repertoire of cadherins, which are critical for BMP-2-induced osteogenic differentiation. J Bone Miner Res, 1998. 13(4): p. 633-44.

40. Andrew B. Yeatts, E.M.G., Fayola F. Fears, John P. Fisher, Human Mesenchymal Stem Cell Position Within Scaffolds Influences Cell Fate During Dynamic Culture. Biotechnology \& Bioengineering, 2012.

41. Goldstein, A.S., Effect of seeding osteoprogenitor cells as dense clusters on cell growth and differentiation. Tissue Eng, 2001. 7(6): p. 817-27.

42. Lavery, K., et al., BMP-2/4 and BMP-6/7 differentially utilize cell surface receptors to induce osteoblastic differentiation of human bone marrow-derived mesenchymal stem cells. J Biol Chem, 2008. 283(30): p. 20948-58.

43. Xiao, Y.-T., L.-X. Xiang, and J.-Z. Shao, Bone morphogenetic protein. Biochemical and Biophysical Research Communications, 2007. 362(3): p. 550-553.

44. Itoh, F., et al., Promoting bone morphogenetic protein signaling through negative regulation of inhibitory Smads. Vol. 20. 2001. 4132-4142.

45. Chen, G., C. Deng, and Y.-P. Li, TGF- $\beta$ and BMP Signaling in Osteoblast Differentiation and Bone Formation. International Journal of Biological Sciences, 2012. 8(2): p. 272-288.

46. Kundu, J., et al., An additive manufacturing-based PCL-alginate-chondrocyte bioprinted scaffold for cartilage tissue engineering. J Tissue Eng Regen Med, 2013.

47. Chimal-Monroy, J. and L. Diaz de Leon, Expression of N-cadherin, N-CAM, fibronectin and tenascin is stimulated by TGF-beta1, beta2, beta3 and beta5 during the formation of precartilage condensations. Int J Dev Biol, 1999. 43(1): p. 59-67.

48. Gatherer, D., et al., Expression of TGF-beta isoforms during first trimester human embryogenesis. Development, 1990. 110(2): p. 445-460.

49. Heldin, C.H., K. Miyazono, and P. ten Dijke, TGF-beta signalling from cell membrane to nucleus through SMAD proteins. Nature, 1997. 390(6659): p. 465-71. 
50. Schmierer, B. and C.S. Hill, TGFbeta-SMAD signal transduction: molecular specificity and functional flexibility. Nat Rev Mol Cell Biol, 2007. 8(12): p. 970-82.

51. Mohan, N., et al., Continuous gradients of material composition and growth factors for effective regeneration of the osteochondral interface. Tissue Eng Part A, 2011. 17(21-22): $p$. 2845-55.

52. Singh, M., et al., Microsphere-based seamless scaffolds containing macroscopic gradients of encapsulated factors for tissue engineering. Tissue Eng Part C Methods, 2008. 14(4): p. 299309.

53. Dormer, N.H., et al., Osteochondral Interface Regeneration of Rabbit Mandibular Condyle With Bioactive Signal Gradients. Journal of Oral and Maxillofacial Surgery, 2011. 69(6): p. e50-e57.

54. Dormer, N.H., et al., Osteochondral Interface Tissue Engineering Using Macroscopic Gradients of Bioactive Signals. Annals of biomedical engineering, 2010. 38(6): p. 2167-2182.

55. Zara, J.N., et al., High doses of bone morphogenetic protein 2 induce structurally abnormal bone and inflammation in vivo. Tissue Eng Part A, 2011. 17(9-10): p. 1389-99.

56. James, A.W., et al., A Review of the Clinical Side Effects of Bone Morphogenetic Protein-2. Tissue Eng Part B Rev, 2016. 22(4): p. 284-97.

57. Tannoury, C.A. and H.S. An, Complications with the use of bone morphogenetic protein 2 (BMP-2) in spine surgery. Spine J, 2014. 14(3): p. 552-9.

58. Milind Singh, C.B., and Michael S. Detamore, Strategies and Applications for Incorporating Physical and Chemical Signal Gradients in Tissue Engineering. TISSUE ENGINEERING: Part B, 2008. 


\section{Valorization}

The research conducted into the laboratories of research centers and universities might often be disconnected from the business world. This generates often, difficulties in valorizing the work conducted in these laboratories and put the scientists in a challenging position to develop their idea into a working marketable concept [1]. The process of identifying a valuable idea and translate it into a product, which has an impact on the market and the society, is called "technology transfer" or "knowledge valorization". Universities, companies and government organizations are supported by technology transfer offices. Their role is to identify which research has potential commercial interest and how to best develop and use it [2].

In the present thesis, the effect of scaffolds with in-built gradient on human mesenchymal stem cells (hMSCs) differentiation was analyzed in order to mimic the gradual transition from the subchondral bone toward the cartilage tissue found at the joint surface.

Some scaffolds designs showed more promising results, which may be easier to valorize. In chapter 6 the use of scaffolds presenting axial pore architecture gradients showed a potential in the effect of promoting the differentiation of hMSCs. In the following section I will propose some ways in which the findings of this chapter could be valorized. In particular, I will focus on the social and clinical relevance of our research. Subsequently, I will focus on the novelty of the scaffold design and its possible applications.

\section{Social and clinical relevance}

The osteochondral tissue is located at the end of the long bones and allows the transition from the hard mineralized bone to the soft and highly hydrated cartilage. It is possible to identify different zones within this transition tissue. Shortly, from the bottom to the top of the osteochondral tissue we first encounter the subchondral bone, followed by calcified cartilage. From this point, the cartilage starts dividing into the deep zone, the middle zone and the articular surface. This stratification 
translates in a gradient variation of characteristics such as stiffness, cellularity and biochemical composition.

Osteoarthritis (OA), the degeneration of the osteochondral tissue, is estimated to be worldwide the fourth leading cause of disability and the most common selfreported cause of disability in activities of daily living [3, 4]. According to the Centers for Disease Control and Prevention (CDC), about 27 million people in the United States have osteoarthritis. The disease is a progressive degeneration of the joint which starts with subtle biochemical changes, leading to a gross cartilage loss and morphological damage of other joint tissues [5]. During the onset of osteoarthritis the collagen matrix becomes more disorganized and the proteoglycan content in cartilage decreases. These changes generate a major increase in water content. Since the protection effect of proteoglycan is lost, collagen fibers begin to undergo degradation generating a domino effect, which can lead to inflammation and pain [6-8]. Current treatments rely on surgery and present several drawbacks, and sometimes as in case of osteotomy are helping in pain relief and retardation of OA onset, but are not effective in the long term [9].

\section{Novelty}

Rapid prototyping has emerged in the last decades in the field of tissue engineering and regenerative medicine for its versatility and possible applications. The scaffolds are generated in a layer by layer manner ensuring a fully interconnected pore structures with tunable pore size and shape. Additionally, structural parameters like the diameter, distance and orientation of the struts can be tuned during the production process [10]. Typically, the fiber deposition pattern is used to vary the direction of the fibers every layer, leading to pores with honeycomb shaped obtained after several layers [11]. In chapter 6 the pattern 0-a0 - $a$ was used (for example $0-45-0-45$ ) in order to have a pore defined by 2 subsequent layers and have a constant pore shape for 6 layers. The novelty of this simple design is the generation of a reproducible repetitive unit which, within a scaffold zone, is constant in all the directions. With such a constant structure all the cell residing in a scaffold portion experience the same geometrical cues, allowing 
an easy interpretation of the relationship between pore geometry and hMSC differentiation.

The link between the novel design and its possible application results from the behavior of hMSCs residing in the different scaffold sections. Under chondrogenic conditions squared pores (0-90) enhanced the differentiation toward a chondrogenic lineage, whereas moving toward the portion of the scaffold with the elongated rhomboidal shape this expression decreased. Conversely under osteogenic conditions, osteogenic markers expression was prominent in the long rhomboidal pores (0-15) and decreased moving toward the square pore region. Usually the differentiation tests are conducted under osteogenic or chondrogenic conditions in vitro. hMSCs in our scaffolds followed the just described differentiation pattern also when cultured in a media containing both the osteogenic and chondrogenic stimuli.

\section{Possible application}

One of the most promising and effective biological based therapies for OA is autologous chondrocyte implantation, where the chondrocytes of the patient are harvested from a biopsy of health cartilage, expanded ex vivo and re-implanted in the defect following debridement. Despite reported good outcomes, this technique is highly time consuming due to the chondrocyte expansion from the cartilage biopsy. Additionally the patients have to go through several operations.

With our pore shape gradient scaffold, the number of intervention and time can be considerably reduced. We propose the removal of the damaged cartilage and subchondral bone portion with a biopsy. From the CT scan of the area to be removed we acquire the code to print a scaffold with the same shape of the removed portion with the pore shape gradient along the $z$ axis. The scaffold can be produced under aseptic conditions or sterilized immediately after manufacturing. The scaffold will be placed in the hole generated by the biopsy with the $0-15$ pores in contact with the subchondral bone and the squared pores at the cartilage surface. After implantation, the scaffold will be filled with the bone marrow that can be accessed through microfracturing of the underlying subchondral bone. This will 
be the source of the hMSCs, which over time will populate the scaffold. The osteogenic and chondrogenic signals necessary to the hMSCs to differentiate will be provided by the surrounding tissues. This one step operation will reduce the time and discomfort caused by the harvesting of chondrocytes and their expansion before the actual reparative operation can be performed. Before translating this technique to the hospital and the market, additional studies in small and big animal models must be performed. Afterwards, additional clinical studies on sensitive patients should also be done to compare our scaffold with current therapies.

1. Miron-Shatz, T., et al., Promoting business and entrepreneurial awareness in health care professionals: lessons from venture capital panels at medicine 2.0 conferences. J Med Internet Res, 2014. 16(8): p. e184.

2. Caldera, A. and O. Debande, Performance of Spanish universities in technology transfer: An empirical analysis. Research Policy, 2010. 39(9): p. 1160-1173.

3. Fransen, M., et al., The epidemiology of osteoarthritis in Asia. Int J Rheum Dis, 2011. 14(2): p. 113-21.

4. Mannoni, A., et al., Epidemiological profile of symptomatic osteoarthritis in older adults: a population based study in Dicomano, Italy. Annals of the Rheumatic Diseases, 2003. 62(6): p. 576-578.

5. Sanchez-Adams, J., et al., The mechanobiology of articular cartilage: bearing the burden of osteoarthritis. Curr Rheumatol Rep, 2014. 16(10): p. 451.

6. Bollet, A.J. and J.L. Nance, Biochemical Findings in Normal and Osteoarthritic Articular Cartilage. II. Chondroitin Sulfate Concentration and Chain Length, Water, and Ash Content. Journal of Clinical Investigation, 1966. 45(7): p. 1170-1177.

7. Brocklehurst, R., et al., The composition of normal and osteoarthritic articular cartilage from human knee joints. With special reference to unicompartmental replacement and osteotomy of the knee. J Bone Joint Surg Am, 1984. 66(1): p. 95-106.

8. Venn, M. and A. Maroudas, Chemical composition and swelling of normal and osteoarthrotic femoral head cartilage. I. Chemical composition. Annals of the Rheumatic Diseases, 1977. 36(2): p. 121-129.

9. Katz, J.N., B.E. Earp, and A.H. Gomoll, Surgical Management of Osteoarthritis. Arthritis care \& research, 2010. 62(9): p. 1220-1228. 
10. Woodfield, T.B., et al., Design of porous scaffolds for cartilage tissue engineering using a three-dimensional fiber-deposition technique. Biomaterials, 2004. 25(18): p. 4149-61.

11. Dietmar W. Hutmacher Thorsten Schantz, I.Z., Kee Woei Ng, Swee Hin Teoh, Kim Cheng Tan, Mechanical properties and cell cultural response of polycaprolactone scaffolds designed and fabricated via fused deposition modeling <1097-4636(200105)55-2-203--AID-JBM1007-3.0.pdf>. J Biomed Mater Res, 2000. 


\section{Samenvatting}

Voor het onderzoek beschreven in dit verslag is met uit beenmerg verkregen humane mesenchymale stromale cellen (hMSCs) gewerkt, aangezien deze cellen tijdens een klinische operatie via bijvoorbeeld microfracturen met een scaffold gecombineerd zouden kunnen worden. De cellen werden gezaaid op AM scaffolds met verschillende gradiënten waarna hun differentiatie in bot- of kraakbeencellen werd gekarakteriseerd om de effectiviteit van de ontworpen 3D gradiënten te bepalen. De scaffolds werden op verschillende manieren geproduceerd om gradiënten te creëren op basis van poreusheid, geometrie, fysisch-chemische eigenschappen en bioactieve moleculen.

In hoofdstuk 1 wordt de osteochondrale overgangszone gepresenteerd als een weefsel met een gradiënt, waarvan vanuit het perspectief van biologische groei wordt uitgelegd hoe de weefselcompositie varieert vanaf het subchondrale bot tot aan het kraakbeen. Ook wordt de bestaande literatuur over de meest recente scaffold-gebaseerde behandelmethodes welke gradiënt structuren gebruikten beschreven.

In hoofdstuk 2 werd de differentiatie van de hMSCs naar botcellen geanalyseerd nadat ze gekweekt waren in een scaffold met een gradiënt van poriegrootte. Alkaline fosfatase (ALP) activiteit, de expressie van genetische kenmerken Runx2, bot sialoproteïne, osteocalcine, osteopontine, en ALP, en de aanwezigheid van gemineraliseerde deposities werden geanalyseerd om te bekijken of de gradiënt hier een voordelig effect op had vergeleken met homogene scaffolds zonder gradiënt. Dezelfde eigenschappen werden geanalyseerd om te karakteriseren of cellen in compartimenten met een bepaalde gradiënt differentieerden naar botcellen.

In hoofdstuk 3 werd de differentiatie van hMSCs naar kraakbeencellen geanalyseerd in een gradiënt met dezeflde structurele eigenschappen als die beschreven in hoofdstuk 3 . Als kenmerken voor differentiatie naar kraakbeencellen werden glycosaminoglycaan (GAG) productie en genetische kenmerken sox 9 , 
aggrecaan, en collageen type II gebruikt. Ook hier werd het effect van de scaffoldgradiënten op hMSC differentiatie naar kraakbeen vergeleken met die van scaffolds met een homogene poriegrootte en binnen de gradiënt compartimenten.

In hoofdstuk 4 was de poreusheid gradiënt gecreëerd in de radiale richting. Pijpbeenderen tonen ook een gradiënt in de radiale richting, met van buiten naar binnen eerst een laag compact bot, dan een laag trabeculair bot en in het midden het beenmergkanaal. Scaffolds met een vergelijkbare poreusheid als deze drie regio's werden geplot en het differentiatieprofiel van de hMSCs naar kraakbeencellen werd geanalyseerd. De mechanische eigenschappen van de scaffolds werden gekarakteriseerd en hMSC differentiatie naar kraakbeen geanalyseerd door middel van genetische en biochemische kenmerken. Histochemische kleuring en röntgen diffractie werden gebruikt om mineralisatie aan te tonen.

In hoofdstuk 5 werd de hMSC differentiatie in scaffolds met een porievorm gradiënt over de z-as onderzocht. De porievorm gradiënt scaffolds werden geplot door de hoek tussen de vezels binnen opeenvolgende lagen te variëren. Een hoek tussen vezels van 0-90 leidde tot vierkante poriën, een hoek van 0-45 tot ruitvormige poriën, en hoeken van $0-30$ en $0-15$ tot ruitvormige poriën met een steeds kleinere kleinste diagonaal en grotere grootste diagonaal. De differentiatie van hMSCs naar bot- en kraakbeencellen werd geanalyseerd op biochemisch en genetisch gebied. Kenmerken voor bot- en kraakbeenvorming geanalyseerd in gradiënt scaffolds werden vergeleken met scaffolds met een homogene poriegrootte en binnen een individuele scaffold als functie van porievorm.

In hoofdstuk 6 werden scaffold met fysisch-chemische gradiënten geanalyseerd. Door 3 materialen voor 1 scaffold te gebruiken werden gradiënten in stijfheid en oppervlakte-energie gerealiseerd. Deze scaffolds werden eerst gekarakteriseerd en de gradiënten werden geprofileerd over de z-as. De hMSC differentiatie naar bot- en kraakbeencellen werd geanalyseerd als reactie op deze gradiënten. 
In hoofdstuk 7 werden nanoborstels gebruikt als verbinding tussen het scaffoldoppervlak en bioactieve eiwitten. $\mathrm{Na}$ deze toepassing werd een simpele methode geïntroduceerd om een gradiënt in bioactieve moleculen te verkrijgen. Om de biologische activiteit van zulk een gradiënt te bewijzen werd fibronectine aan scaffolds toegevoegd zodat hier cellen aan konden hechten na de applicatie van zowel een radiale als axiale gradiënt.

In hoofdstuk 8 werden BMP-2 en TGF- $\beta 3$ aan de scaffolds gehecht. Het effect van deze toevoegingen op hMSC differentiatie werd vergeleken met dat van cellen op een scaffold zonder deze toevoegingen maar met dezelfde toevoegingen opgelost in het kweekmedium. Scaffolds met een gradiënt van deze toevoegingen werden gecreëerd en hun effect of hMSC differentiatie geëvalueerd.

In hoofdstuk 9 werden alle scaffolds beschreven in dit verslag met elkaar vergeleken. Ook worden vervolgstudies voorgesteld om de kennis van scaffolds met een gradiënt voor kraakbeen regeneratie te vergroten, zodat het in de toekomst mogelijk nuttig kan zijn in de klinische setting. 


\section{Curriculum Vitae}

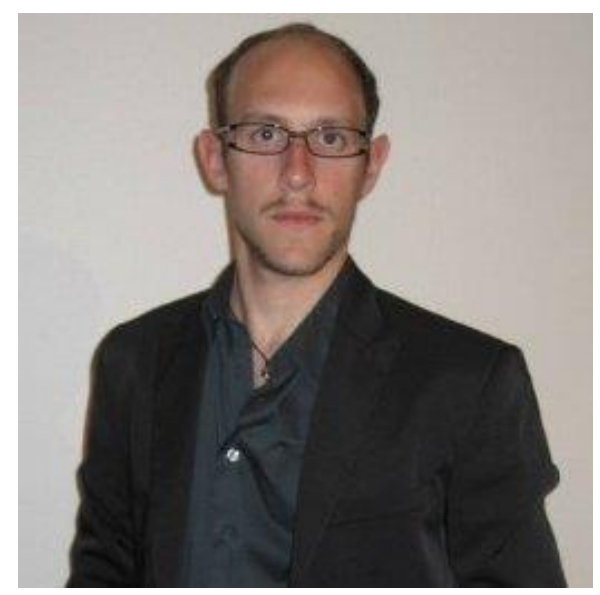

Andrea Di Luca was born in Trieste (Italy) on the $27^{\text {th }}$ July 1984. In 2006 he obtained his Bachelor degree in Biotechnology at Universitá degli studi di Trieste. He continued his study path in the same University and in 2008 he obtained his master degree in Medical Biotechnology (molecular medicine curricula) with the thesis "Nanocomposite systems based on polysaccharides and metal nanoparticles for biomedical applications". Immediately after he was enrolled as research intern in Network of Excellence for Functional Biomaterials, National University of Ireland Galway. In 2010 he started his PhD at Twente University, MIRA Institute for Biomedical Technology and Technical Medicine, Tissue Regeneration group, Enschede under the supervision of Clemens van Blitterswijk and Lorenzo Moroni working on the regeneration of the osteochondral interface by using gradient scaffolds. In 2015 he was hired as research technician at Eindhoven Technical University, Department of Biomedical Engineering. 


\section{Acknowledgment}

Eventually here we are, last rows and steps of a long path that led to this thesis. This work would not have been the same without the contribution of so many people, colleagues, friends, family. I will try to thank all of you here.

First of all I would like to thank Prof Clemens van Blitterswijk to have given me the opportunity to join his group and work close by so many brilliant people. A huge thank to Prof Lorenzo Moroni. Lore, you decided to hire me on top of the doubt you had. I really hope to have payed back the trust you have granted me. You have been more than just a supervisor; helping me not only on the scientific but also on the more personal and human growth. Please keep doing this to your future students.

A special thanks to Prof Julius Vancso and Dr. Edmondo Benetti for have shared the project and the help provided with advices and meetings. I would also thank the STW committee which accompanied me with regular meeting and direct support Dr. Theo Flipsen, Dr. Clayton Wilson and Ing. Hendrik John. I would thank Prof Mark Post, Dr. René van Donkelaar, Prof Rastogi Sanjay, Prof Peter Kessler, Dr. Jeroen van den Beuken for having accepted to evaluate my thesis and being part of my committee.

Of course I want to give my big thank to my paranimphs. Starting with the outsider, Fabri. Te la faccio facile in italiano anche se il tuo inglese é molto buono (finché stai fuori dall'Olanda). Cosa dirti che non ti ho detto nella scorsa tesi? Tu ci sei. Sempre. Amico, supporto, il fratello maggiore che non ho mai avuto, padrino di mia figlia. Semplicemente grazie di tutto quello che c'é stato e che ha da venire.

Now Michel. Long ago when we met downstairs at TR, we looked so different and I couldn't forecast how deep would have grown this collaboration. You have been an excellent collaborator, now you are my best friend here in the Netherlands and the person which I can always count on for a help, a word... a beer. I will never forgot 
when Alessia was in the hospital, you just jumped on your car drove 2 hours to keep me company for 2 hours and drive back. You are great, thanks dude!

Tim, you are responsible for having unleashed the most nerdy part of me by introducing me to Magic, I loved our hours spent by having my ass kicked by your decks, with an ice cold beer to further enjoy it. Likewise you have been a very good colleague and a great beach volley teammate, Timboooooo thanks a lot for everything!

Mamma, papá grazie di avermi supportato sempre e comunque. Voi eravate lí a spronarmi, consigliarmi e non mi avete mai deluso come genitori, siete fantastici e come sempre avete dato un grandissimo contributo ai miei traguardi. Marco, Lollo e Stefano, grazie del supporto e dei momenti passati insieme quando possibile, l'unica cosa che rimpiango della vita all'estero é non poter passare piú tempo con voi.

Ora vorrei ringraziare la mia famiglia, Giulia e Alessia. Giulia che dire? Un interview a Birmingham é terminata con un bacio e il dottorato a Enschede con la nostra Alessia. Sei stato tutto per me qui, collega, amica, e tra alti e bassi guarda cosa abbiamo costruito. Senza di te il piú grande traguardo della mia vita non sarebbe stato possibile. Alessia sei l'orgoglio di papá, buona parte di questa tesi é stata scritta con te quando mi stavi su un braccio. Sei la figlia che ogni padre vorrebbe avere. Grazie di essere come sei e di averci lasciato dormire nei primi mesi!

A special thanks to all the people that have been more than just colleagues but nice friends Mijke, Paul, Milou, Marcel, Erik, Lorena, Niloofar, Febryiani, Parthiban, Giuseppe, Alessia, Carlos, David, Joana, Zyrian, Ivan, Miriam, Marzia, Natalie and Jingwei. Thanks for the drinks, parties, the salsa lessons and the beach volley. $E$ ovviamente grazie a Mattia (Údin) e Laura rifugio di "procrastination", aciditá quanto basta e buoni consigli, sempre con caffettino annesso. I would also thank all the people I shared the house in the first year of the $\mathrm{PhD}$ and Harambee volley group. 
Thanks to my students Lionel, Nic and Alessia. Lionel for sharing first a project and then the house. Nic for the "nerdy" combination of science and Magic. Alessia you have been a brilliant student, part of this thesis have been possible only thank to your independency and patience. Thanks to you and Beppe for the lunches, coffees and the movies during plotting and PCR sessions. Thanks also to Barbara for the nice collaboration.

Thanks to the Biofabrication group, Anne, Wim, Paul, Febryiani, Carlos, Ivan, Giuseppe, Sara and Hongling; and to all the TR group Pamela, Jan, Roman, Anouk, Tom, Bernke, Bjorn, Aliaksei, Nathalie, Bach, Ana, Angand, Charlene, Ana, Hugo and Max.

The thesis was mainly written in Eindhoven, therefore I would like to thank Prof Bouten and Dr. Dankers for giving me the possibility to work in their group and of course the whole STEM and ICMS group and of course the tech team and my office mates Marloes, Marina, Sylvia, Roy, Marc and Moniek.

And I cannot miss to thank the Eindhoven gang Paul, Mijke, Stefano, Karin, Mieke, Julian, Tina and Mayur, thanks for the nice time spent together from parties to last minute organized dinners and drinks. Let's keep them going on!

And thanks to all those people that were there too short to develop a real friendship or collaboration, but that anyway took part of this journey from youth to adulthood. 


\section{Selected publication within this thesis:}

"Toward mimicking the bone structure: design of novel hierarchical scaffolds with a tailored radial porosity gradient"

Di Luca A, Longoni A, Criscenti G, Mota C, van Blitterswijk CA, Moroni L Biofabrication, 2016 October

"Tuning Cell Differentiation into a 3D Scaffold Presenting a Pore Shape Gradient for Osteochondral Regeneration"

Di Luca A, Lorenzo-Moldero I, Mota C, Lepedda A, Auhl D, Van Blitterswijk C, Moroni L Advanced Healthcare Materials, 2016 July

"Influencing chondrogenic differentiation of human mesenchymal stromal cells in scaffolds displaying a structural gradient in pore size"

Di Luca A, Szlazak K, Lorenzo-Moldero I, Ghebes CA, Lepedda A, Swieszkowski W, van Blitterswijk CA, Moroni L.

Acta Biomaterialia, 2016 May

"Gradients in pore size enhance the osteogenic differentiation of human mesenchymal stromal cells in three-dimensional scaffolds"

Di Luca A, Ostrowska B, Lorenzo-Moldero I, Lepedda A, Swieszkowski W, van Blitterswijk $C A$, Moroni L

Scientific Reports, 2016 March

"Surface energy and stiffness discrete gradients in additive manufactured scaffolds for osteochondral regeneration."

Di Luca A, Longoni A, Criscenti G, Lorenzo-Moldero I, Klein-Gunnewiek M, Vancso J,van Blitterswijk CA, Mota C, Moroni L

Biofabrication. 2016 February

"The osteochondral interface as a gradient tissue: from development to the fabrication of gradient scaffolds for regenerative medicine"

Di Luca A, van Blitterswijk CA, Moroni L

Birth Defects Research Part C: Embryo Today: Reviews. 2015 March

"Creeping Proteins in Microporous Structures: Polymer Brush-Assisted Fabrication of 3D Gradients for Tissue Engineering" 
Klein Gunnewiek $M^{*}$, Di Luca $A^{*}$, Bollemaat $H$, van Blitterswijk CA, Vancso GJ, Moroni $L^{*}$, Benetti EM*

Advanced healthcare materials, 2015 February

\section{Other publications:}

"Hybrid Polycaprolactone/Alginate Scaffolds Functionalized with VEGF to Promote de Novo Vessel Formation for the Transplantation of Islets of Langerhans"

Marchioli G, Di Luca A, de Koning E, Engelse M, Van Blitterswijk CA, Karperien M, Van Apeldoorn AA, Moroni $L$

Advanced Healthcare Materials, 2016 July

"Influence of internal pore architecture on biological and mechanical properties of 3D fibre deposited scaffolds for bone regeneration."

Ostrowska B, Di Luca A, Moroni L, Swieszkowski W

Journal of Biomedical Material Research part A, 2016 April

"Triphasic scaffolds for the regeneration of the bone-ligament interface."

Criscenti G, Longoni A, Di Luca A, De Maria C, van Blitterswijk CA, Vozzi G, Moroni L.

Biofabrication, 2016 January

"Stem-Cell Clinging by a Thread: AFM Measure of Polymer-Brush Lateral Deformation"

Klein Gunnewiek M, Ramakrishna S, Di Luca A, Vancso G, Moroni L, Benetti E Advanced Material interfaces, 2015 December

"Amphiphilic Beads as Depots for Sustained Drug Release Integrated into Fibrillar Scaffolds"

Gaharwar AK, Mihaila SM, Kulkarni AA, Patel A, Di Luca A, Reis RL, Gomes ME, van Blitterswijk C, Moroni L, Khademhosseini A

Journal of controlled release, 2014 August

"Thin polymer brush decouples biomaterial's micro-/nanotopology and stem cell adhesion"

Klein Gunnewiek M, Benetti EM, Di Luca A, van Blitterswijk CA, Moroni L, Vancso GJ Langmuir. 2013 November 
"Poly(N-isopropylacrylamide)-poly(ferrocenylsilane) dual responsive hydrogels: Synthesis, Characterization and antimicrobial applications"

Sui X, Feng X, Di Luca A, van Blitterswijk CA, Moroni L, Hempenius MA, Vancso GJ Polym. Chem., 4, 337 - 342 (2013)

"Fabrication and antimicrobial effects of silver nanoparticle-poly $(\mathrm{N}$ isopropylacrylamide)-poly(ferrocenylsilane) hydrogel composites"

Sui X, Feng X, Di Luca A, van Blitterswijk, CA, Moroni, L, Hempenius, MA, Vancso GJ 2012 MRS Spring Meeting

"Controlled surface initiated polymerization of poly $(\mathrm{N}$-isopropylacrylamide $)$ on polycaprolactone substrates for regulating cell attachment and detachment" Klein Gunnewiek M, Di Luca A, Sui X, van Blitterswijk CA, Moroni, L, Vancso, GJ Israel Journal of Chemistry. 2012 April

"Stability and Cell Adhesion Properties of Poly( $\mathrm{N}$-isopropylacrylamide) Brushes with Variable

Grafting Densities"

Sui X, Di Luca A, Klein Gunnewiek M, Kooij S, van Blitterswijk C, Moroni L, Hempenius M, Vancso J

Australian Journal of Chemistry. 2011 September 